\title{
Actin-Myosin Crossbridge Binding and Regulation Studied Using Force Spectroscopy
}

\author{
A Dissertation \\ Presented to the Faculty of \\ The School of Engineering and Applied Science \\ University of Virginia
}

\begin{abstract}
In Partial Fulfillment
Of the Requirements for the Degree

Doctor of Philosophy in Biomedical Engineering
\end{abstract}

Amy Michelle Clobes

May 2014 


\section{APPROVAL SHEET}

The dissertation

is submitted in partial fulfillment of the requirements

for the degree of

Doctor of Philosophy

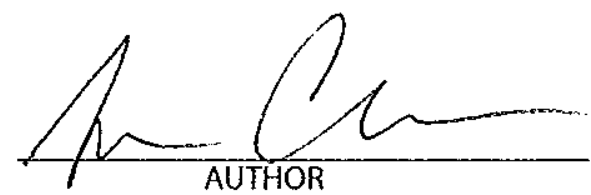

The dissertation has been read and approved by the examining committee:

William H. Guilford, Ph.D. Advisor

Silvia S. Blemker, Ph.D.

Edward H. Egelman, Ph.D.

Brent A. French, Ph.D.

Jeffrey J. Saucerman, Ph.D.

Accepted for the School of Engineering and Applied Science:

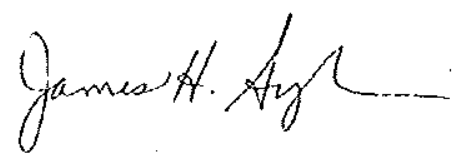

Dean, School of Engineering and Applied Science

May 


\section{Acknowledgements}

I would like to start by thanking my advisor, Dr. William Guilford. To him I owe a terrific amount of gratitude for his help and guidance in the lab, in teaching, and in life. He has been an excellent mentor and truly a friend.

I would next like to thank the members of my dissertation committee: Drs. Jeffrey Saucerman, Brent French, Silvia Blemker, and Edward Egelman. Their guidance, thoughtful discussion, support, and encouragement were instrumental throughout the course of this project.

I want to thank past and present members of the Molecular Biomechanics Laboratory. You have made this experience what it is on a daily basis. I would particularly like to thank Vijay Rao and Jeremy Snook, as well as the many undergraduate students who have given life, learning, and laughter to the lab over the years.

Finally, I want to say thanks to my family and friends. It is you who have unfailingly encouraged and supported me throughout my endeavors.

Thank you! 


\section{Abstract}

Muscle contraction requires a symphony of proteins that act and interact to generate force and movement. Actin and myosin are the essential crossbridge proteins that drive muscle contraction, and they rely on regulatory mechanisms to fine-tune their activity. This regulation can be intramolecular, or it can come from post-translational modifications, or interaction with another protein or small molecule. Because of the myriad of interactions in a sarcomere, it is difficult to discern the presence and significance of each one. Singlemolecule and ensemble in vitro models allow us to isolate the effects of a single protein or post-translational modification to a specific component of the contractile apparatus something that cannot be done in intact fibers or isolated myofibrils. We exploited the capabilities of single-molecule measurements to understand the mechanism of two important regulatory crossbridge interactions.

First, we show that myosin loop 2 internally regulates the crossbridge by acting as a forcedependent inhibitor of the long-lived actomyosin complex. Here, an optical trap was used to measure bond formation and rupture between actin and rigor heavy meromyosin (HMM) with loop 2 intact or tryptically cleaved. When loop 2 was cleaved, actomyosin catch bond behavior was abrogated leaving only a long-lived state - pointing to the integral role of loop 2 and the importance of force in crossbridge regulation.

Cardiac myosin binding protein-C (cMyBP-C) is a sarcomeric protein that directly regulates crossbridge formation and cycling, though its specific binding partner and mechanism of regulation are unknown. Moreover, cMyBP-C activity itself is regulated by phosphorylation and may be regulated by nitrosylation. In order to identify the crossbridge binding partner of cMyBP-C, we measured the force-dependent bond rupture between cMyBP-C and each of the requisite crossbridge components, actin and HMM, individually. We found that neither the bond with actin nor the bond with HMM was regulated by phosphorylation, excluding these as essential crossbridge binding partners for cMyBP-C and disproving the leading 
hypothesis in the field. Further, our work shows that nitrosylation of cMyBP-C does not play a role in regulating actin-myosin interactions.

These studies have allowed us to gain a better understanding of the protein-protein interactions between actin, myosin, and cMyBP-C that are key mechanisms of crossbridge formation and regulation. Together, our data on the functions of loop 2 and cMyBP-C increase our understanding of striated muscle function and may be especially important in understanding pathologies. Hypertrophic cardiomyopathy in particular is directly linked to mutations in $\mathrm{CMyBP}-\mathrm{C}$, and until we have a better understanding of how cMyBP-C regulates crossbridge activity the mechanism of disease will remain unclear. 


\section{Table of Contents}

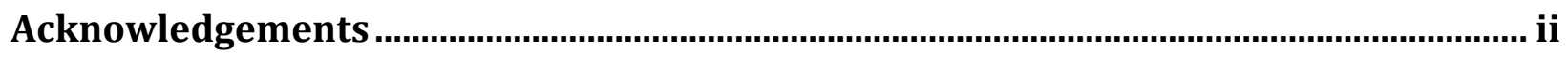

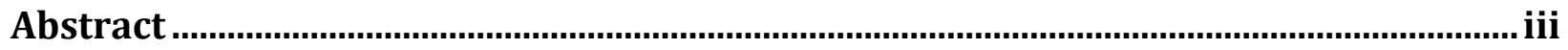

List of Abbreviations.............................................................................................................ii

Chapter 1: Background ……........................................................................................... 1

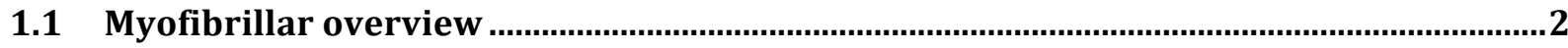

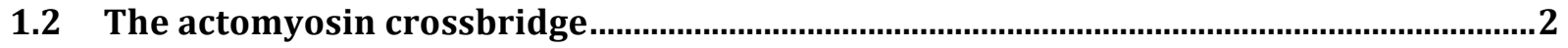

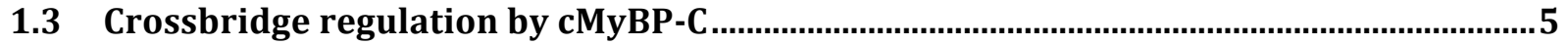

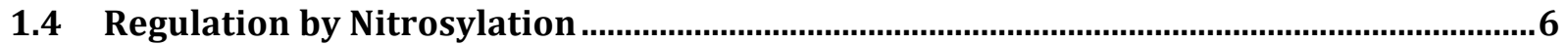

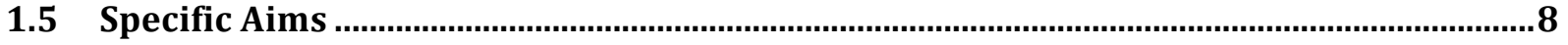

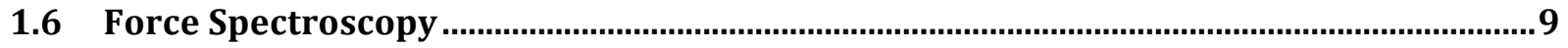

Chapter 2: Loop 2 of myosin is a force-dependent inhibitor of the rigor bond ............12

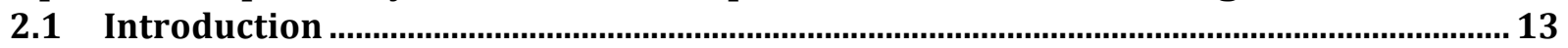

2.2 Materials and Methods ...........................................................................................14

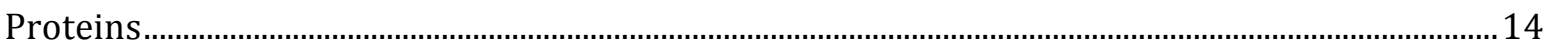

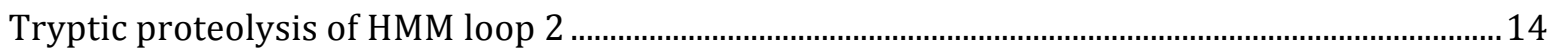

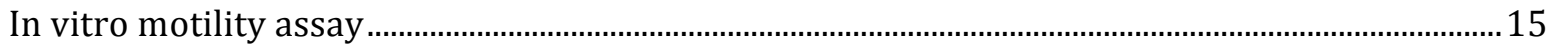

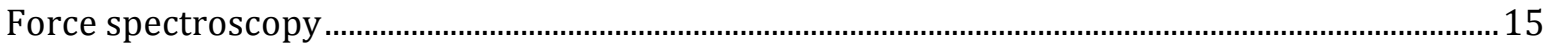

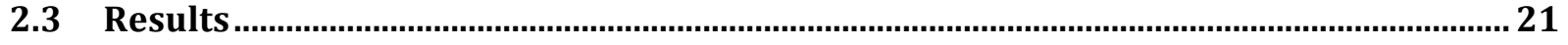

Ionic strength has a modest effect on the actomyosin catch bond ………………………………....22

The actomyosin catch bond is the result of allostery ……………………………………………….....2 24

Loop 2 is required for catch bond behavior........................................................................................26

Load dependent 2D on-rates ................................................................................................................27

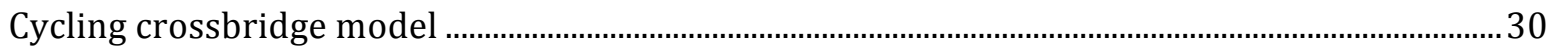

The catch bond increases the crossbridge dissociation rate ...................................................................32

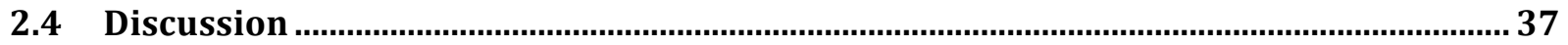

2.5 Conclusion................................................................................................................. 42

Chapter 3: cMyBP-C phosphorylation-dependent crossbridge regulation ....................43

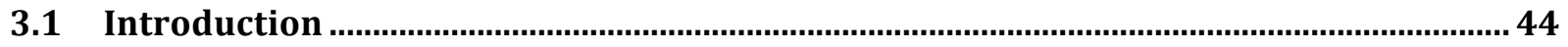

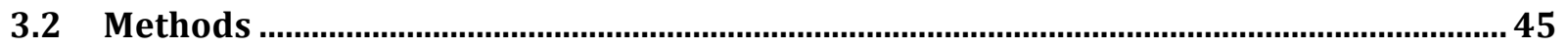

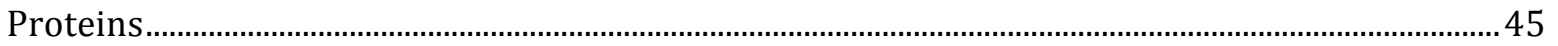

Force spectroscopy …………………………………………………………………………………………....4

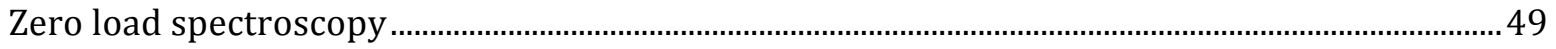

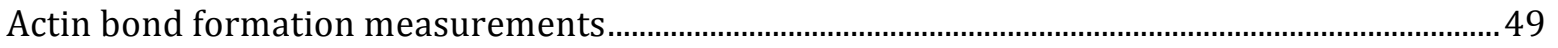

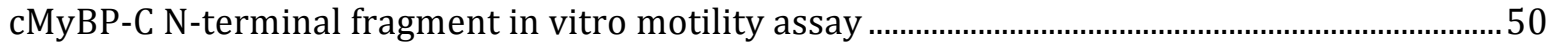

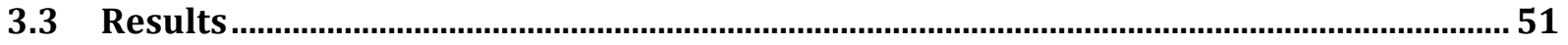

Phosphorylation does not modulate load-dependent cMyBP-C fragment bond lifetimes with

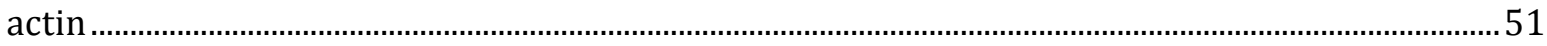

Phosphorylation does not modulate load-dependent cMyBP-C bond lifetimes with HMM ........53

Frequency of bond formation as a function of C0C3 surface density ……………………………....55

Actin binding is unaffected by C0C3 phosphorylation ........................................................................56 


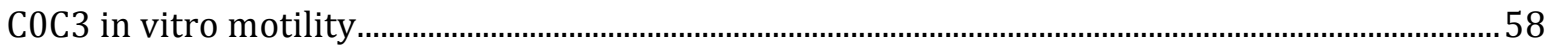

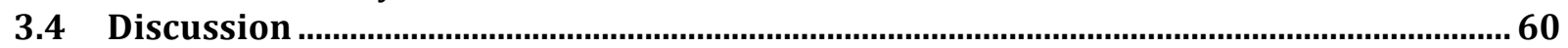

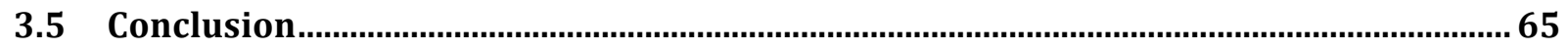

Chapter 4: Exploring the functional effect cMyBP-C nitrosylation.................................67

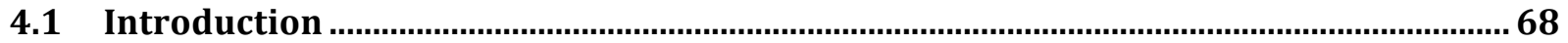

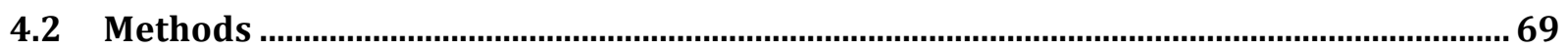

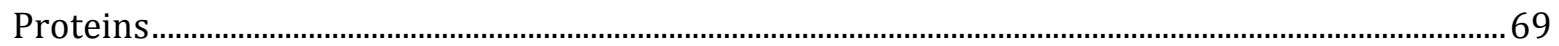

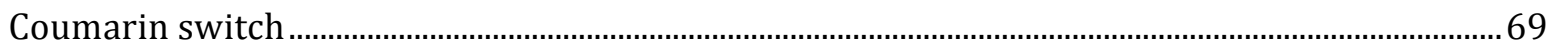

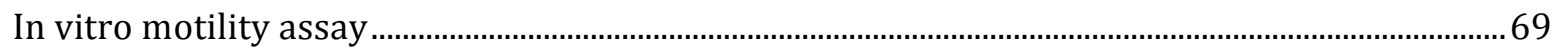

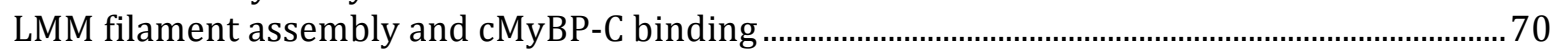

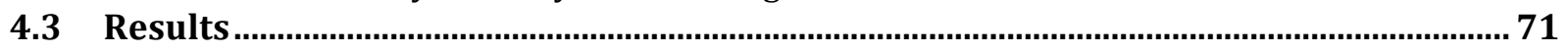

Quantification of cMyBP-C nitrosylation.............................................................................................. 71

Nitrosylation does not affect the ability of cMyBP-C to regulate actomyosin motility ..................72

LMM filament assembly and cMyBP-C binding are unaffected by nitrosylation ............................. 73

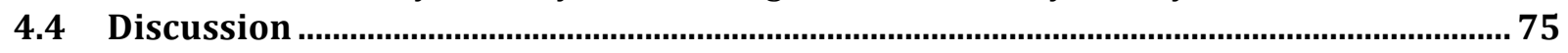

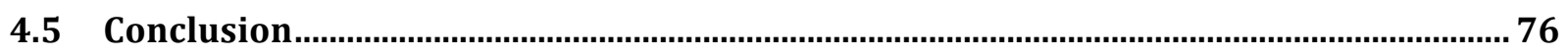

Chapter 5: Conclusions and Future Directions .................................................................. 77

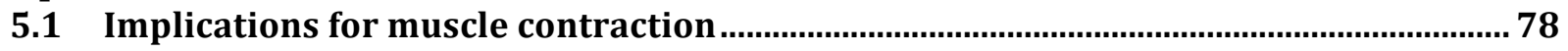

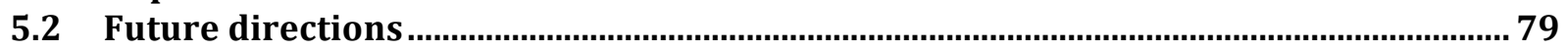

Loop 2 load-dependent regulation of the actomyosin crossbridge ....................................................79

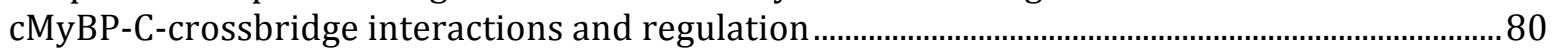

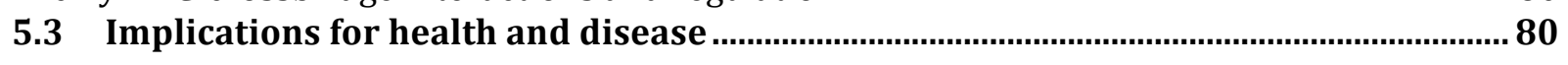

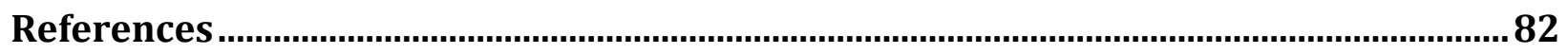

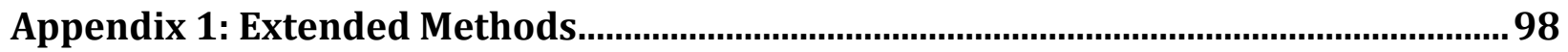

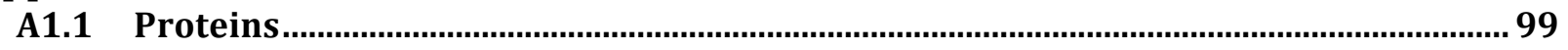

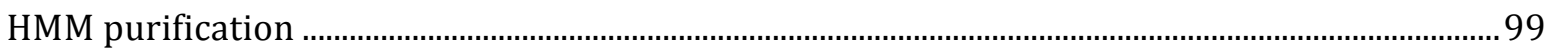

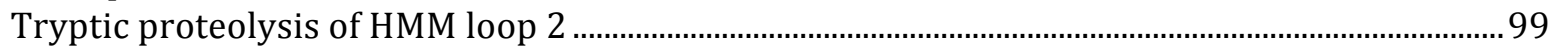

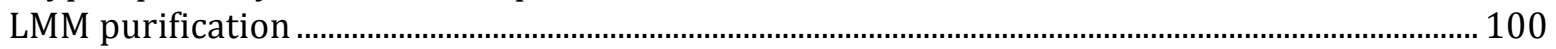

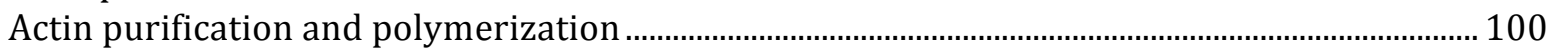

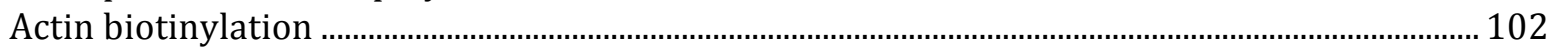

Biotinylated actin microspheres..................................................................................................... 102

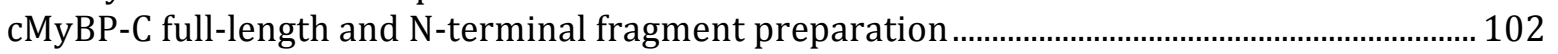

Biotinylated C0C3 and C0C3-4D microspheres............................................................................... 103

A1.2 Actin-HMM Force Spectroscopy and In Vitro Motility ...................................................103

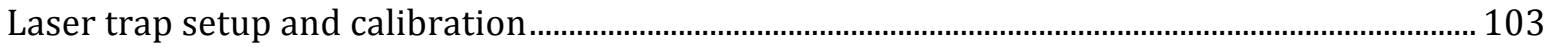

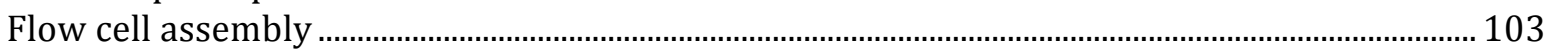

Flow cell loading for actin-HMM force spectroscopy experiments ............................................... 104

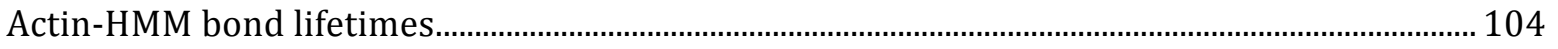

Actin-HMM fraction of bonds surviving ..................................................................................... 106

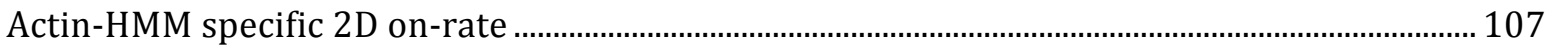

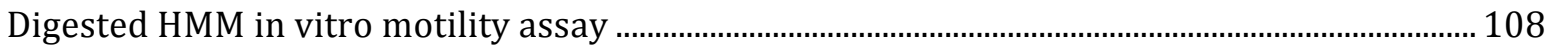

A1.3 cMyBP-C Phosphorylation-Dependent Crossbridge Binding.....................................109

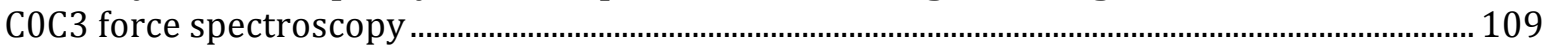

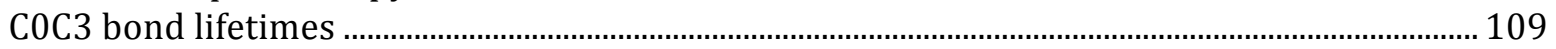

C0C3 zero load force spectroscopy ................................................................................................. 110

Actin-C0C3 bond formation measurements ................................................................................... 110

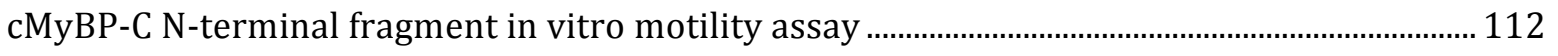

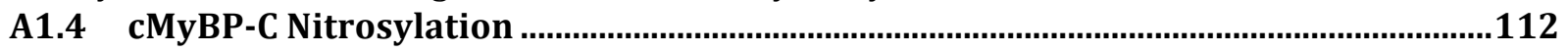


Nitrosylation donors

Coumarin switch assay

Nitrosylation Treatment for cMyBP-C Motility

cMyBP-C nitrosylation and in vitro motility assay

Nitrosylation treatment for CMyBP-C binding to LMM

LMM filament assembly and cMyBP-C binding

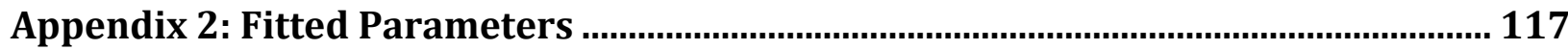

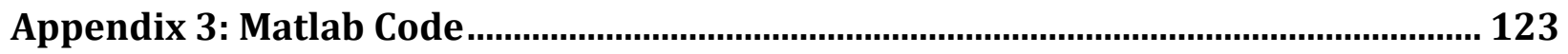

Appendix 4: Matlab Code for Cycling Crossbridge Model............................................... 134 


\title{
List of Abbreviations
}

\author{
AMCA-HPDP \\ N-(6-[7-amin0-4-methylcoumarin-3-acetamido]-hexyl)-3'- \\ (2'pyridyldithio) propionamide \\ AMD \\ ADP-Bound Actomyosin \\ BTEE \\ benzoyl-L-tyrosine ethyl ester \\ C0C3 \\ cMyBP-C Fragment Containing Domains C0 through C3 \\ C0C3-4D \\ Phosphomimetic cMyBP-C Fragment Containing Domains C0 through \\ C3, with Aspartic Acid Replacement of Serines 273, 282, 302, and 307 \\ in the M-motif \\ cMyBP-C Cardiac Myosin Binding Protein-C \\ DEA-N0N0ate 1,1-Diethyl-2-hydroxy-2-nitroso-hydrazine sodium \\ DMSO Dimethyl sulfoxide \\ DTT Dithiothreitol \\ EDTA Ethylene diamine tetraacetic acid \\ EGTA Ethylene glycol tetraacetic acid \\ FHC Familial Hypertrophic Cardiomyopathy \\ GSNO S-Nitrosoglutathione \\ HEPES 4-(2-Hydroxyethyl)-1-piperazineethanesulfonic acid \\ HMM Heavy Meromyosin \\ LMM Light Meromyosin \\ M Myosin \\ MD ADP-Bound Myosin \\ MMTS Methyl methanethiosulfonate
}




$\begin{array}{ll}\text { MOPS } & \text { 3-(N-Morpholino) propanesulfonic acid } \\ \text { MT } & \text { ATP-Bound Myosin } \\ \text { MWCO } & \text { Molecular Weight Cut Off } \\ \text { MyBP-H } & \text { Myosin Binding Protein-H } \\ \text { NHS } & \text { N-hydroxysuccinimide } \\ \text { NO } & \text { Nitric Oxide } \\ \text { PKA } & \text { Protein Kinase A } \\ \text { PMSF } & \text { Phenylmethylsulphonyl fluoride } \\ \text { PPi } & \text { Pyrophosphate } \\ \text { QD } & \text { Quadrant Photodiode Detector } \\ \text { SN0-L-cys } & \text { S-Nitroso-L-cysteine } \\ \text { TPCK } & \text { 1-Chloro-3-tosylamido-4-phenyl-2-butanone } \\ \text { TRITC } & \text { Tetramethyl rhodamine iso-thiocyanate }\end{array}$




\section{Chapter 1: Background}




\subsection{Myofibrillar overview}

Muscle contraction and force generation are driven by myosin's conversion of chemical energy to mechanical work through actin-dependent ATP hydrolysis and allosteric conformational changes (Geeves and Holmes, 2005). While the coupling of nucleotide binding, hydrolysis, and nucleotide dissociation to the power stroke of myosin is reasonably well understood, the molecular-level details of the actin-myosin interactions themselves have not been fully elucidated. The structural details of the actomyosin binding interface remain unknown, including the specific residues involved and the role of a disordered surface loop. Furthermore, other sarcomeric proteins are associated with these two main force-generating components, though their specific interactions with and functional roles in regulation of the crossbridge cycle remain unclear. For example, we have recently shown that tropomyosin, a member of thin filament regulation, directly interacts with myosin in crossbridge binding to regulate its rate of binding, not simply its ability to bind (Rao et al., 2011). Another regulatory protein, cardiac myosin binding protein-C (cMyBP-C) also has direct effects on crossbridge formation and cycling, though its crossbridge binding partner and mechanism of regulation remain unknown. Both tropomyosin and cMyBP-C are phosphoproteins, and like tropomyosin, cMyBP-C interactions with the crossbridge are thought to be phosphorylation-dependent. However, in the case of cMyBP-C the relationship of phosphorylation to binding is unclear. It is obvious that force-bearing intermolecular bonds figure prominently in the sarcomere. We thus seek to gain a better understanding of the protein-protein bonds between actin, myosin, and cMyBP-C and which of these interactions are key mechanisms of crossbridge formation and regulation.

\subsection{The actomyosin crossbridge}

The striated muscle sarcomere contains more than 40 proteins that interact to enable and regulate force-generation (Fraterman et al., 2007). While there is a general macro-level understanding of the machinery and function of a muscle cell, the structural details of how these molecules interact to generate force are unclear. The actomyosin crossbridge is ultimately responsible for muscle contraction by myosin binding to actin and undergoing 
allosteric rearrangement to displace the actin filament. However, there are molecular details of crossbridge cycling, such as the structure of the actomyosin binding interface, that are unresolved.

The initial step in actin-myosin binding is thought to occur through long-range electrostatic interactions, creating a weak collision complex that is followed by isomerization to the attached state and a second isomerization to a rigor-like, strong-binding state (Geeves and Conibear, 1995). Myosin's actin-binding pocket is hydrophobic and is neighbored by the positively charged loop 2 (the "actin binding loop", Figure 1) which is thought to help the myosin head to locate its binding site on actin (Rayment et al., 1993a). Once the initial electrostatic interaction between the loop 2 and actin's myosin binding site is established, binding can proceed to a strongly-bound state through additional hydrophobic and stereospecific interactions (Geeves and Conibear, 1995; Geeves et al., 1984). The actin binding pocket of myosin is also a key element of another allosteric change that the myosin head undergoes in response to actin or nucleotide binding; it is thought to close on binding to actin and to subsequently change shape in response to ADP release and with ATP binding. 


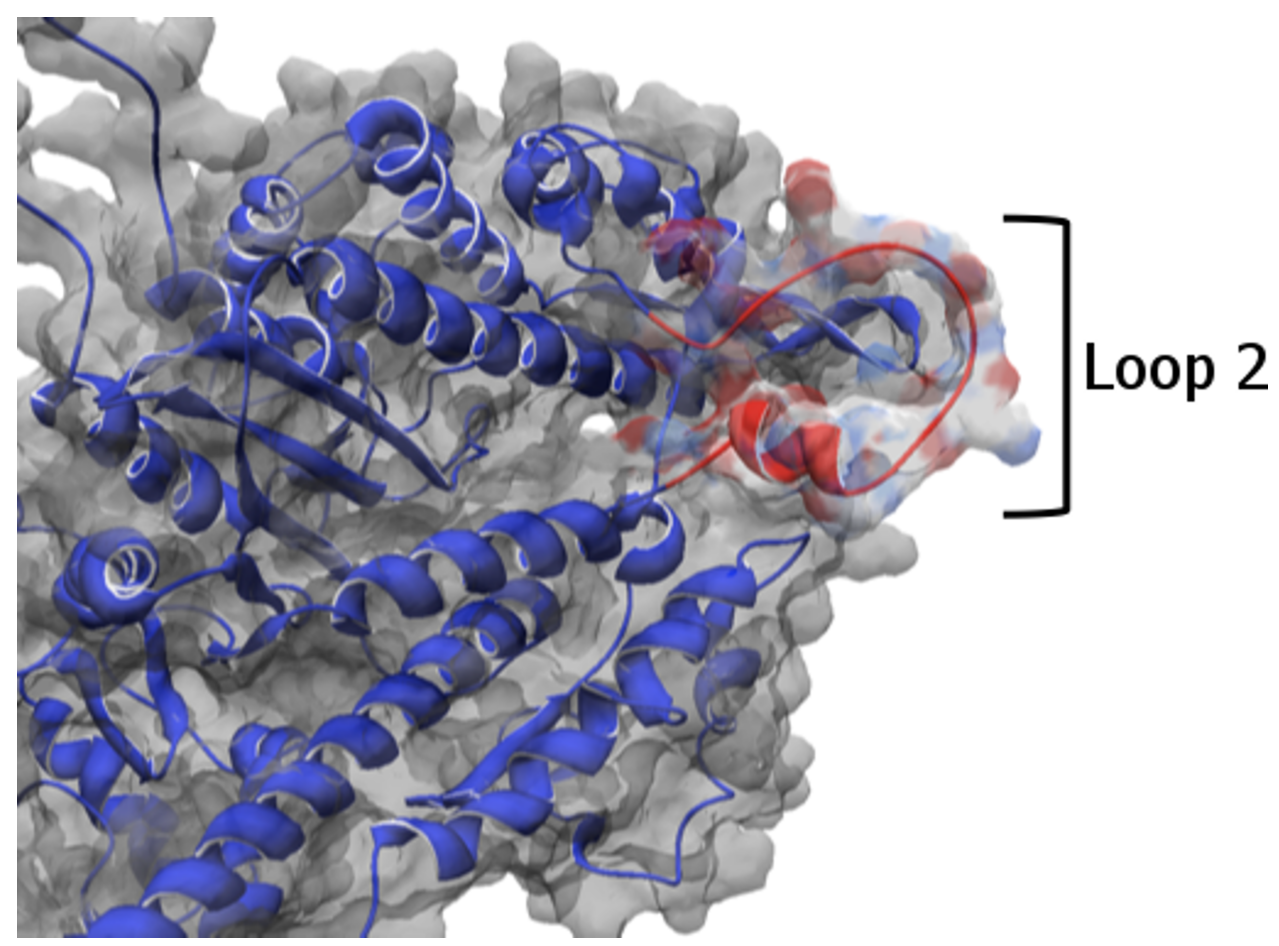

Figure 1: Structure of the myosin head. The charged loop 2 is represented by red ribbon, other myosin residues are blue ribbon.

Loop 2 connects myosin's 50 and 20-K domains (Rayment et al., 1993b). The general function of loop 2 includes the control of actin-activated ATPase activity by altering $\mathrm{V}_{\mathrm{m}}$ and actin affinity (Murphy and Spudich, 2000). The loop varies in length and sequence (Murphy and Spudich, 2000) but is well-conserved among myosins with similar kinetics (Goodson et al., 1999). It is particularly reliant on the presence and sequence of a few key residues rather than its overall charge or length to retain its function in modulating kinetics (Murphy and Spudich, 2000).

Because the loop is flexible, it is unresolved in most myosin II structures except for two from Dictyostelium discoideum (Lorenz and Holmes, 2010). However, crosslinking and digestion studies have shown loop 2 to be part of the actomyosin binding interface (Mornet et al., 1981; Sutoh, 1982). Still, the shape and location of loop 2 after binding is controversial. Molecular dynamics simulations suggest that myosin's loop 2 takes on a distinct conformation upon actin-myosin binding, but its location and interactions differ between studies (Liu et al., 2006; Lorenz and Holmes, 2010). A recent high resolution cryo- 
electron microscopy-based model identifies potential electrostatic interactions between loop 2 on myosin and actin's N-terminus, putting loop 2 at the center of the actin-myosin interface (Behrmann et al., 2012).

\subsection{Crossbridge regulation by cMyBP-C}

Actin and myosin themselves are not the only binding partners present at this crossbridge interface. Tropomyosin is a well-known steric regulator of actomyosin interaction, occupying a position on the actin filament to block myosin's access to binding sites. We have shown through optical force spectroscopy that tropomyosin has an additional binding interface with myosin, accelerating bond formation in some tropomyosin states on the actin filament (Rao et al., 2011).

Another important regulatory protein is $\mathrm{cMyBP}-\mathrm{C}$. The specific function of $\mathrm{cMyBP}-\mathrm{C}$ within the sarcomere is unclear, but it is evident that contraction is directly regulated by cMyBP-C. In the presence of cMyBP-C, cardiac myosin ATPase activity is increased (Yamamoto and Moos, 1983), $\mathrm{Ca}^{2+}$-activated force generation is reduced (Kunst et al., 2000), and actin filament in vitro motility is inhibited (Saber et al., 2008). The activity of cMyBP-C appears to be modulated by phosphorylation. While cMyBP-C phosphorylation does not have a significant effect on ATPase activity (Hartzell, 1985) it relieves inhibition of actin filament motility seen in the presence of dephosphorylated cMyBP-C (Weith et al., 2012a). This regulation of contraction suggests that $\mathrm{cMyBP}-\mathrm{C}$ interacts with the crossbridge proteins in a phosphorylation-dependent manner.

Ablation of cMyBP-C in mice results in significant cardiac hypertrophy and depressed contractile function (Harris et al., 2002). cMyBP-C phosphorylation may be critical both to disease prevention and to maintaining normal myocardial function (Sadayappan et al., 2005). However, the role of N-terminal cMyBP-C appears to be more complicated than a simple phosphorylation-dependent drag on crossbridge motility. It also has been suggested to regulate crossbridge detachment rates through reduced actin-activated ATPase rates (Razumova et al., 2006). cMyBP-C and may also modulate thin-filament regulation. Herron et al. show the ability of a $\mathrm{COC} 2 \mathrm{~N}$-terminal fragment to activate force production in 
skinned ventricular myocytes in the absence of $\mathrm{Ca}^{2+}$, and suggest that it acts via a different activation mechanism than $\mathrm{Ca}^{2+}$ (Herron, 2006). Mun et al. recently confirmed this observation using in vitro motility and found that N-terminal cMyBP-C modulates thin filament activity by physically displacing tropomyosin through an independent molecular mechanism from that which it uses to govern actomyosin contractile speed (Mun et al., 2014).

Two prominent theories have emerged to explain how cMyBP-C regulates the crossbridge. Both are dependent upon cMyBP-C binding to a crossbridge protein and restricting the protein's movement. N-terminal cMyBP-C binds either with actin or with myosin and stops the actin filament from sliding. Skinned fiber and ensemble motility experiments suggest that an interaction with cMyBP-C imposes a load on myosin (Hofmann et al., 1991a, 1991b), however it is unclear whether the load is simply a tether on the actin filament that is being propelled by myosin motor or whether it causes a direct impairment of myosin head movement itself.

Kunst et al. provided evidence that cMyBP-C binds myosin's S2 region, controlling myosin head distance from the filament backbone and its cycling activity that can be relieved by Mmotif phosphorylation (Kunst et al., 2000). There is also structural and functional evidence that cMyBP-C interacts physiologically with the thin filament (Kulikovskaya et al., 2003; Luther et al., 2011; Mun et al., 2014; Weith et al., 2012a, 2012b). Weith et al. have expanded our understanding by using single molecule techniques to show that an N-terminal portion of the M-motif binds stereospecifically with actin and that this binding may be reversible by phosphorylation (Weith et al., 2012a, 2012b). It has even been proposed that phosphorylation may alternate N-terminal cMyBP-C binding between actin and myosin (Pfuhl and Gautel, 2012).

\subsection{Regulation by Nitrosylation}

Nitrosylation, like phosphorylation, is a post-translational modification that has been shown to regulate muscle activity. Protein nitrosylation primarily occurs when nitric oxide (NO) reacts with a cysteine residue in the presence of an electron acceptor to yield a S- 
nitrosothiol. It may also occur through the transfer of NO from a nitrosothiol to a thiol through a nitroxyl disulfide intermediate (Perissinotti et al., 2005). NO is generated in vivo by nitric oxide synthases and has been recognized as a signaling molecule influencing muscle contraction and contractility both indirectly through activation of guanylyl cyclase and directly by acting on proteins through nitrosylation.

NO donors have been shown to decrease $\mathrm{Ca}^{2+}$ sensitivity, ATPase activity, shortening velocity, and isometric force in skeletal (Andrade et al., 1998; Galler et al., 1997; Perkins et al., 1997) and cardiac (Shoji et al., 1999) filaments in vitro. These data highlight the potential for nitrosylation as a regulatory feature of muscle, since a significant portion of proteins in the force generation apparatus are nitrosylatable in vitro (Kohr et al., 2011). However, the functional effects of NO on myofibrillar apparatus remain to be understood as it is difficult to parse out which of the individual proteins in these muscle fiber preparations is responsible for the observed effects.

The identities of endogenously affected skeletal and cardiac myofibrillar proteins was recently probed in vitro using S-nitroso-L-cysteine (SNO-L-cys) as an endogenous nitric oxide donor (Guilford and Filo, 2010). Nitrosylatable skeletal muscle proteins included myosin heavy chain, myosin binding protein- $\mathrm{H}$, actin, troponin $\mathrm{I}$, the slow and fast isoforms of myosin essential light chain, and myosin regulatory light chain. In cardiac muscle, these proteins included myosin heavy chain, $\mathrm{cMyBP}-\mathrm{C}$, actin, troponin I, and myosin essential light chain. Of these, the functional effects of myosin nitrosylation are the only that have thus far been published. Interestingly, nitrosylation of myosin reduces actin motility velocity, yet this is accompanied by increased myosin force production in single molecule assays such as those that are used in the present work (Evangelista et al., 2010). These data suggest that nitrosylation acts as a "gear shift" for myosin, switching the motor to a state of lower force generation but higher velocity.

A recent nitrosoproteome of cardiac myofilaments bolsters the evidence that cMyBP-C is able to be nitrosylated (Figueiredo-Freitas et al., 2013), but the functional consequences of cMyBP-C S-nitrosylation remain to be tested. Singling out the effect of cMyBP-C 
nitrosylation would make an important contribution to our understanding of the mechanisms of NO regulation of cardiac contractility.

\subsection{Specific Aims}

We have identified two important, unresolved regulatory mechanisms of crossbridge formation and cycling - actin-myosin interactions that comprise the binding interface itself, and cMyBP-C that interacts with one or both of the essential crossbridge proteins. Myosin's loop 2 is thought to be responsible for seeking out its oppositely-charged binding site on actin. This occurs through long-range electrostatic interaction brings the two molecules together, but these residues may also play a role in determining the actomyosin bond lifetime through a weak- to strong-binding transition. cMyBP-C is also implicated in crossbridge regulation through binding to actin or to myosin at the crossbridge interface. Through its crossbridge binding cMyBP-C is thought to impose a viscous drag, impeding crossbridge movement. Phosphorylation of cMyBP-C is evidenced to modulate its effect on the crossbridge. cMyBP-C can also be nitrosylated, though the functional effects of this modification of cMyBP-C's ability to regulate the actomyosin crossbridge are yet unknown. We thus seek to gain a better understanding of the protein-protein interactions between actin, myosin, and cMyBP-C that are key mechanisms of muscle crossbridge formation and regulation.

\section{Aim 1: Examine the interaction between actin and myosin at their binding interface to determine prerequisites for catch bond behavior.}

(A) Actomyosin has been shown to act as a catch bond, though this catch bond behavior may be an artifact of the sub-physiologic ionic strength conditions used in the experiment. We will test the hypothesis that actomyosin catch bond behavior occurs in vitro at physiologic ionic strength.

(B) Myosin's loop 2 is a component of the binding interface that is thought to be responsible for electrostatic initiation of actomyosin binding. We will test the hypothesis that intact loop 2 is required for catch bond behavior. 
(C) We will use a deterministic model to predict the effect of the catch bond on crossbridge cycling.

\section{Aim 2: Understand how cMyBP-C and its post-translational modification by phosphorylation and nitrosylation change cMyBP-C interactions with actin and myosin.}

(A) We will test the hypothesis that cMyBP-C acts as a phosphorylation-reversible viscoelastic drag on the actin filament in vitro by measuring bond lifetimes between actin and phosphomimetic N-terminal cMyBP-C. We will also test the hypothesis that the bond lifetimes between cMyBP-C and myosin will inversely mirror those of actin with phosphomimetic and unphosphorylated cMyBP-C.

(B) cMyBP-C can by nitrosylated. We hypothesize that the function of cMyBP-C can be regulated by nitrosylation in vitro by modifying either its bond with actin or its bond with the myosin filament backbone.

\subsection{Force Spectroscopy}

Force spectroscopy experimentally determines the time it takes a bond to rupture over a variety of loads or the rupture force under a particular loading condition. Force spectroscopy techniques can be used to measure intra- and intermolecular bonds and can even discern the properties of a single chemical bond. Traditional methods, such as stop flow and in vitro motility make ensemble rather than single molecule measurements. In force spectroscopy, proteins are coupled to a surface that constrains them to approximately two-dimensional motion and the concentration of proteins on the surface allows control of the number of interacting molecules. Thus single-molecule interactions can be measured.

There are three main tools for performing force spectroscopy techniques - the atomic force microscope (AFM), the biomembrane force probe, and the laser trap. Each can directly measure the mechanical properties of a single bond on the scale of nanometer 
displacements and piconewton forces. Each tool consists of a probe that can be bound to one protein or molecule, and displacements of the probe allow measurements of the loaddependent rate with which the molecule interacts with a molecule on the opposing surface. The stiffness of the probe, akin to the stiffness of a spring, relates its displacement to a distance proportional to the force applied according to Hooke's law.

The use of force spectroscopy has led to many important discoveries in molecular mechanics over the last 20 years. The Evans group pioneered force spectroscopy using the biomembrane force probe to study the bond between biotin and streptavidin (Merkel et al., 1999). They showed how force spectroscopy could be used to parse out individual energy barriers within a single intermolecular bond. The Zhu group was also at the forefront of advancing force spectroscopy techniques. They used AFM to directly observe catch bond behavior for the first time in selectins (Marshall et al., 2003), a phenomena that was previously only theoretical (Dembo et al., 1988). Beyond mapping out binding rates and energy landscapes of intermolecular bonds, force spectroscopy has also been used to study the dynamics of molecular motors and the conformational transitions of proteins (Block, 1995; Cluzel et al., 1996; Finer et al., 1994). Single-molecule force spectroscopy is uniquely able to reveal the energy barrier to unfolding or dissociation as well as conformational states (Guo and Guilford, 2006; Merkel et al., 1999; Rief et al., 1997) that are often not observable by conventional methods of structural biology.

Here we used force spectroscopy to measure the intermolecular protein-protein interactions of the muscle crossbridge using a laser trap. In our approach molecules are restricted to approximately planar movement, thereby allowing single actomyosin bonds in our experiments to be examined at physiologic ionic strength. This is similar to their physiologic arrangement where actin and myosin are part of filaments that constrain their free motion to two dimensions. Where solution studies rely on protein diffusion to find their binding partners and on the proteins having a high enough association rate to form bonds, optical force spectroscopy is able to hold the proteins within their binding range so that they can associate to form bonds. Actin and myosin have a low association rate at physiologic ionic strength so the molecules are unlikely to form bonds when freely 
diffusing in solution. The laser trap allows us to simulate this arrangement and thus enabled us to measure the crossbridge binding interface between actin and myosin, as well as with the regulatory cMyBP-C. We aimed to identify the physiological features of the binding interface responsible for initiating bond formation through weak binding, as well as the force dependence of both the association and the dissociation of the bond. We also applied this method to determining the crossbridge binding partner of cMyBP-C and its responsiveness to post-translational modifications. 
Chapter 2: Loop 2 of myosin is a force-dependent inhibitor of the rigor bond 


\subsection{Introduction}

Skeletal muscle actomyosin has been shown to behave as a catch bond in the ADP and rigor states (Guo and Guilford, 2006) and in both the presence and absence of the actin regulatory protein tropomyosin (Rao et al., 2011). Catch bonds increase in lifetime with applied force, up to a critical force value beyond which bond lifetime falls. Catch bonds stand in contrast to slip bonds, which decrease in lifetime with increasing load in an intuitively obvious way. Actomyosin catch bond behavior is though to arise from a force dependent transition from a short- to a long-lived bond state, while slip bonds have only a single bound state. In actomyosin the maximum bond lifetime occurs close to the isometric force that is generated by a single myosin molecule (Guo and Guilford, 2006) suggesting that catch bond function is tuned to maximize bond lifetime during isometric contractions. Others have suggested that the actomyosin catch bond assists in aggregate formation and initial ordering from disorganized actin and myosin networks (Inoue and Adachi, 2013).

Here we tested the hypothesis that myosin's loop 2 alters the mechanics of rigor actomyosin bond formation and rupture, and specifically that it is involved in catch bond behavior. To do this we used force spectroscopy - the dynamic application of force to single molecules or single intermolecular bonds to reveal energy barriers to unfolding or dissociation. Force spectroscopy can reveal conformational and binding states that cannot be resolved by conventional methods of structural biology (Rao et al., 2011). In this instance an optical trap was used to measure the binding rate and the bond lifetimes between heavy meromyosin (HMM) and actin over a range of compressive and tensile loads applied perpendicular to the filament axis. These measurements were performed at both low and physiologic ionic strength. Measurements of intact HMM were compared to those when loop 2 was enzymatically cleaved in order to determine the contributions of loop 2 to binding and unbinding. Our data suggest that loop 2 is a force-dependent inhibitor of a long-lived bound state of actomyosin. 


\subsection{Materials and Methods}

\section{Proteins}

HMM and F-actin were purified from rat skeletal muscle and F-actin was biotinylated and coupled to streptavidin-coated microspheres as described in Appendix 1, Section A1.1.

Tryptic proteolysis of HMM loop 2

HMM was selectively digested at the actin-binding loop 2 (cleaved HMM) as described in Bobkov et al. (1996) with minor modifications. Briefly, HMM was dialyzed against $0.02 \mathrm{M}$ $\mathrm{KCl}, 0.02 \mathrm{M}$ Tris- $\mathrm{HCl}$ and $1 \mathrm{mM}$ DTT at $\mathrm{pH}$ 7.4. To protect loop 1 from digestion, the ionic strength was increased to $0.5 \mathrm{M} \mathrm{KCl}$ and ATP was added to $6 \mathrm{mM}$ (Mocz et al., 1984). TPCKtreated trypsin (proteolytic activity 0.08 BTEE units/mg; Sigma-Aldrich T1426) was added to HMM to a final concentration of $0.05 \mathrm{mg} / \mathrm{ml}$ and incubated on ice for 6 minutes. Digestion was stopped using soybean trypsin inhibitor type 1-S (Sigma-Aldrich T9003) at a molar ratio of 3:1 to trypsin. Cleaved HMM was used within 48 hours of digestion, and then discarded.

Digestion was assessed by electrophoresis on NuPAGE 12\% Bis-Tris Gel in MOPS running buffer and gels were stained using SimplyBlue ${ }^{\mathrm{TM}}$ Safe Stain (Invitrogen, Carlsbad, CA). The molecular weight of fragments was determined by comparison to SeeBlue $®$ Plus2 Protein Standard (Invitrogen) using the AlphaEaseFC (Alpha Innotech Corp., San Leandro, CA) molecular weight tool. As in previous experiments digestion of HMM mainly resulted in two fragments, the N-terminal fragment of S1 and the combined C-terminal region of S1 fragment and S2 (Bálint et al., 1975; Bobkov et al., 1996) (Figure 2). Other contaminating fragments were present in low amounts. 


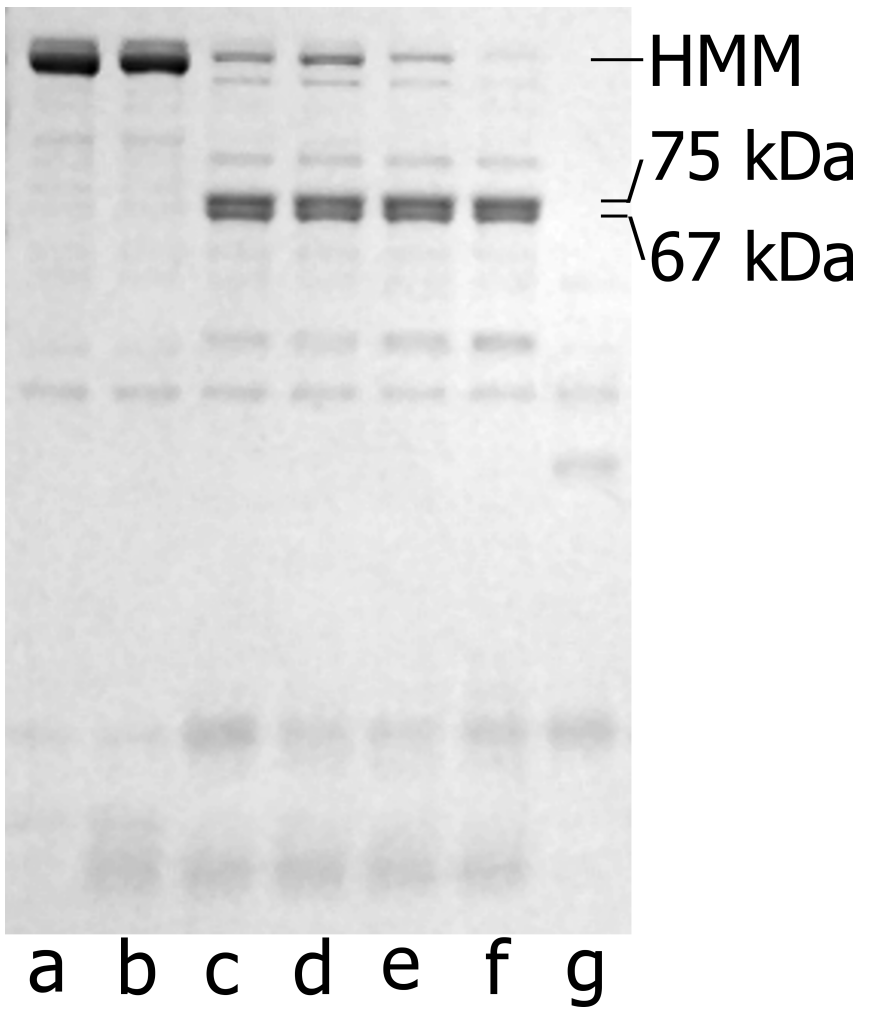

Figure 2: Representative tryptic digestion of HMM. Lanes: (a) HMM prepared by chymotryptic digestion of myosin. (b) Soybean trypsin inhibitor and HMM. (c) HMM pre-incubated with soybean trypsin inhibitor before addition of trypsin. Lanes d-f show HMM digestion by trypsin quenched with soybean trypsin inhibitor after 2 (d), 4 (e), and 6 (f) minutes. (g) HMM digestion by trypsin without inhibitor, which was used as a control. Note that all samples were stored on ice overnight following addition of inhibitor.

In vitro motility assay

In vitro motility was performed with intact, cleaved, or fully digested (no trypsin inhibitor) HMM as described in Appendix 1, Section A1.2.

\section{Force spectroscopy}

The laser trap used in these experiments was the same as described in Guilford et al. with minor modifications (Guilford et al., 2004). The laser is a $25 \mathrm{~W}, 1020 \mathrm{~nm}$ fiber laser (SPI Lasers, Santa Clara, CA), and the digital signal processor has been replaced by a fieldprogrammable gate array (National Instruments, Austin, TX) with a user interface created in LabVIEW (National Instruments). Back focal plane interferometry was used to measure 
the position of a trapped microsphere relative to the trap center, from which measurements of displacement and force were calculated. The temporal resolution of the detection system is $150 \mathrm{kHz}$. The sensitivity of the interferometer and the trap stiffness were calibrated by the step response method (Dupuis et al., 1997; Svoboda and Block, 1994) and by fits to the power spectral density (Allersma et al., 1998).

The experimental setup for laser trap experiments is as follows (Figure 3). Coverslips were coated with 3-10 $\mu \mathrm{m}$ glass spheres (targets) and then coated with nitrocellulose were prepared as in Guo and Guilford (2006) and assembled into flow cells. HMM was incubated in the flow cell for 1 minute. The flow cell was then blocked with $1 \mathrm{mg} / \mathrm{ml} \mathrm{BSA}$ in actin buffer for 1 minute and washed with actin buffer. A suspension of actin buffer and actincoated microspheres was added to the flow cell. An oxygen scavenger system $(0.125 \mathrm{mg} / \mathrm{ml}$ glucose oxidase, $0.0225 \mathrm{mg} / \mathrm{ml}$ catalase, $2.87 \mathrm{mg} / \mathrm{ml}$ glucose) and $0.01 \mathrm{mM}$ DTT were included in the actin buffer and the microsphere suspension. The flow cell was placed onto a piezoelectric microscope stage (nPoint Inc., Middleton, WI). 


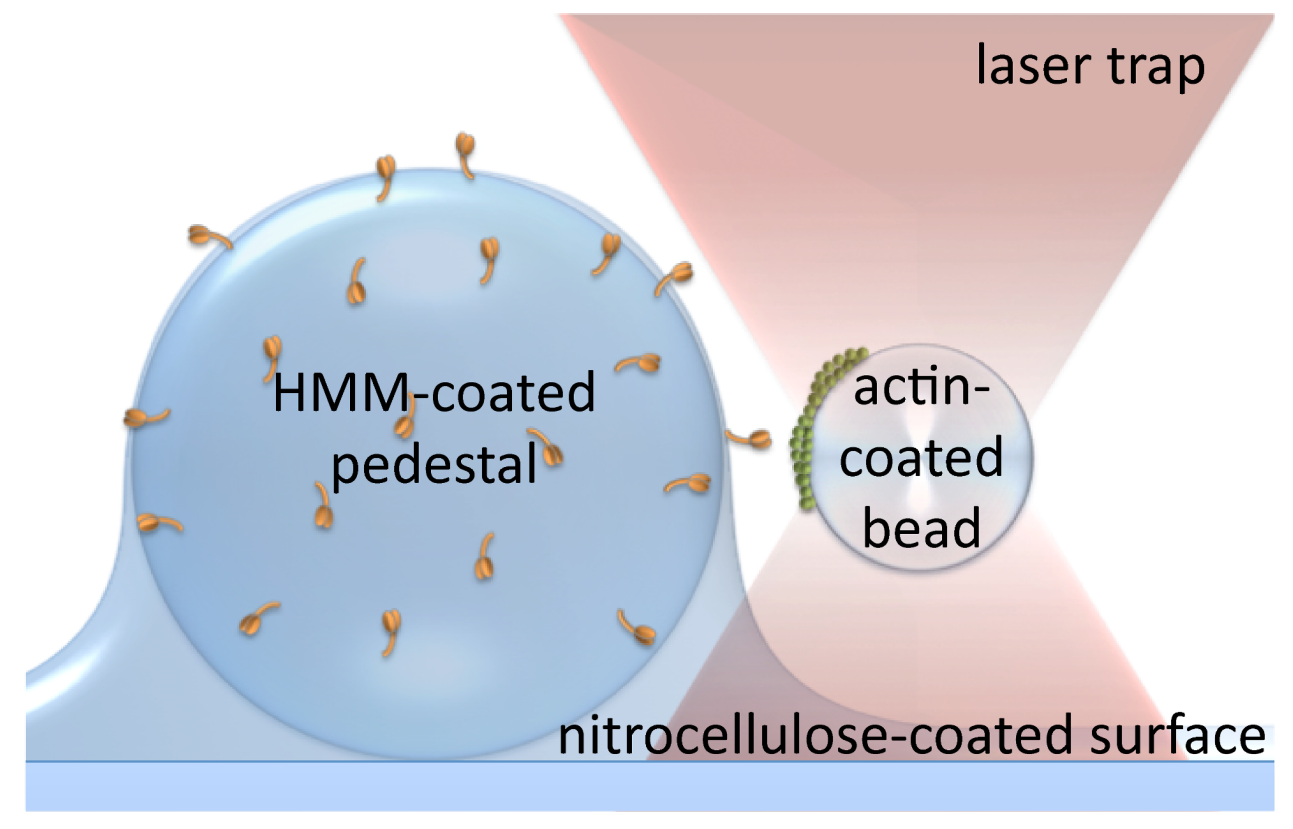

Figure 3: Experimental set up. A microsphere coated in actin is captured in the laser trap. The laser is rapidly stepped toward a nitrocellulose-covered glass bead coated in HMM, bringing the molecules within binding range. The actomyosin bond is loaded by rapidly stepping the laser trap away from the HMM-coated target. The load on the bond is directed perpendicular to the axis of the filament.

All experiments were performed in the absence of nucleotide with intact or cleaved HMM in the presence of $0.025 \mathrm{M} \mathrm{KCl}$ (low ionic strength) or $0.145 \mathrm{M} \mathrm{KCl}$ (physiologic ionic strength). Actin-coated microspheres were captured in a laser trap and brought into contact with an HMM-coated target for 1 second (Figure 4). The concentration of HMM used to coat the target depended on the experiment - $10 \mu \mathrm{g} / \mathrm{ml} \mathrm{HMM}$ for bond rupture experiments, or $80 \mu \mathrm{g} / \mathrm{ml} \mathrm{HMM} \mathrm{for} \mathrm{bond} \mathrm{formation} \mathrm{experiments.} \mathrm{The} \mathrm{laser} \mathrm{trap} \mathrm{was}$ stepped $200 \mathrm{~nm}$ away from the target and held there for 1 second before repeating this cycle. The initial separation between the trapped microsphere and the target was random, resulting in random compressive and tensile loads. Over a large number of experiments this resulted in a range of random step loads being applied to actin-HMM bonds using the laser trap. Bond formation was accompanied by the trapped microsphere remaining in contact with the target after the laser trap was stepped away from it. Bond rupture was accompanied by return of the microsphere to its original position away from the target. The time to bond formation was defined as the time elapsed between stepping the laser 
toward the HMM-coated target and the observed bond formation. Bond lifetime was defined as the time elapsed between stepping the laser away from the HMM-coated target and bond rupture. The tensile load experienced by a bond was calculated from the stiffness of the laser trap and the distance a microsphere moved to return to the center of the laser trap. Details of individual laser trap experiments, including the determination of loads, are given in the results section. 


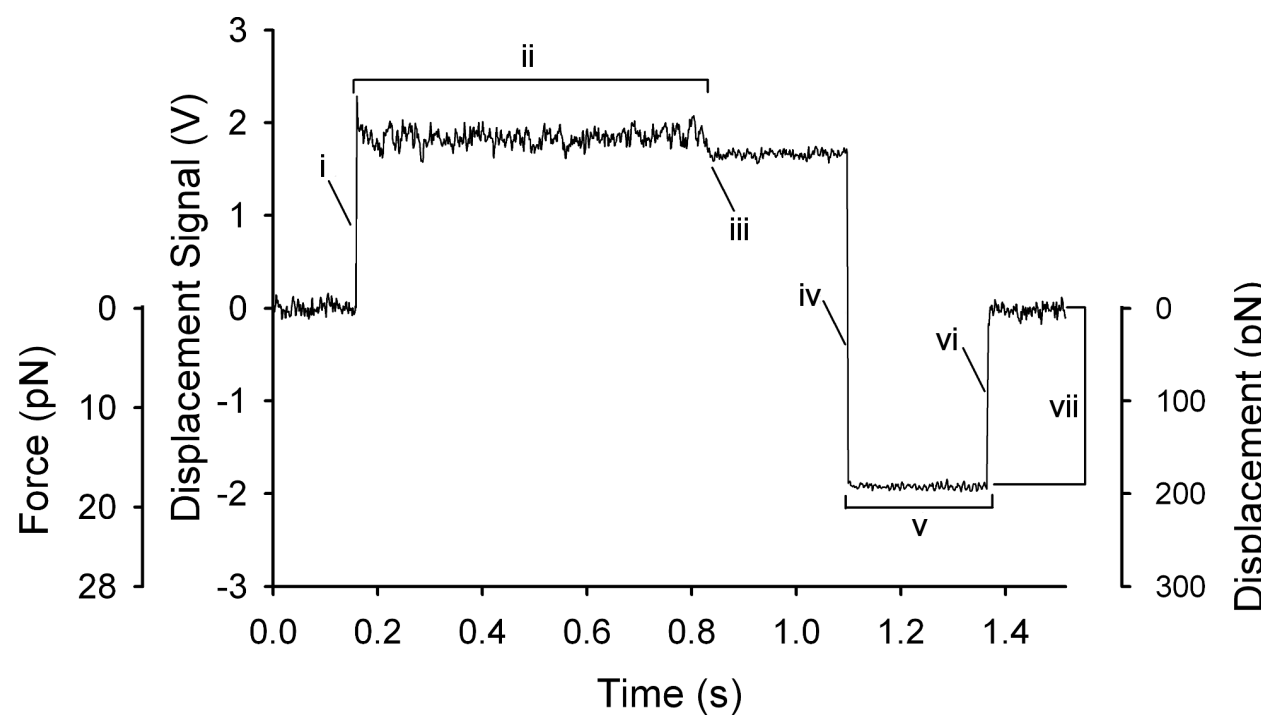

Figure 4: A representative data trace collected for an intact HMM-coated target. (i) The actin-coated microsphere was stepped $200 \mathrm{~nm}$ toward the stationary HMM-coated target which is visible as a jump in the displacement signal. (ii) The molecules were allowed time to form a bond. (iii) $t_{b}$, the time elapsed from when the microsphere was stepped toward the target and when a bond formed (time to bond formation) was identified by a slight shift in the displacement signal and a decrease in Brownian motion. (iv) The bond was then loaded perpendicular to the axis of the filament by stepping the laser $200 \mathrm{~nm}$ away from the HMM-coated target which is seen as a second jump in the displacement signal in the opposite direction of the original shift. In bond rupture measurements, (v) the resulting bond lifetime was measured as (iv) the time from when the laser was stepped away from the target to (vi) the time that the bond ruptured and the microsphere returned to the center of the laser trap. Bond rupture is characterized by the displacement signal returning to the original baseline accompanied by a return to the original level of Brownian motion in the displacement signal. (vii) The tensile load experienced by a bond was calculated based upon the stiffness of the laser trap and the sensitivity of the quadrant photodiode detector. In bond formation experiments, (vii) the compressive load between the actin-coated microsphere and the HMM-coated target was calculated as the difference between distance the laser was stepped and the distance the actin-coated microsphere actually moved, multiplied by the stiffness of the laser. The second $y$-axis, Force $(\mathrm{pN})$, is the product of the displacement signal (V), the stiffness $(\mathrm{pN} / \mathrm{nm})$ and sensitivity $(\mathrm{nm} / \mathrm{V})$ calibrations specific to this example data trace. Displacement signal values $>0$ are not shown because the laser is refracted by the target when the laser is nearby and therefore the stiffness and sensitivity calibrations measured far from the target do not apply in that position. Laser trap stiffness is $0.10 \mathrm{pN} / \mathrm{nm}$ for the sample data trace. 
In sum, the parameters that we can measure using optical force spectroscopy include: the compressive force on a bond as it forms, the time to bond formation, the tensile force applied to a bond, and the bond lifetime at that tension.

We are confident that our data measure the binding interactions between a single actin filament and a single HMM molecule. The surface density of HMM is controlled such that it is sparse enough to allow single-molecule interactions occur. If multiple bonds do occur, the individual rupture events can be observed in the displacement signal and these data are not included in our data set.

Loads were applied perpendicular to the axis of the filament. The minimum bond duration that can be measured by our laser trap system is $62 \mu \mathrm{s}$, which is the time it takes a microsphere under a representative $7 \mathrm{pN}$ force to travel the shortest distance at which it would be outside the range of the noise, given a 99\% confidence interval. Bonds shorter than 4 ms were not measured.

Bond lifetimes were divided into $2 \mathrm{pN}$ bins, within any one of which the lifetimes were exponentially distributed. $95 \%$ confidence intervals of the mean of the exponentially distributed bond lifetime data were calculated in each $2 \mathrm{pN}$ force bin using the equation

$$
\theta \in\left(\frac{2 \sum_{i=1}^{n} X_{i}}{\chi_{\alpha / 2,2 n}^{2}}, \frac{2 \sum_{i=1}^{n} X_{i}}{\chi_{1-\alpha / 2,2 n}^{2}}\right)
$$

where $X_{i}$ is an exponentially distributed variable with mean $\theta$ of $n$ total measurements and $\alpha=0.05$ (Ross, 2009).

Bond lifetimes at near-zero load were measured as in Guo, et al. (2006) by holding an actincoated microsphere adjacent to an HMM-coated target and allowing bonds to form and break under low, semi-random loads (Figure 5). Bond formation was identified by a decrease in Brownian motion accompanied by a small shift in baseline, and bond rupture 
by a reversal of those changes. Fits to power spectral density were used to calibrate the laser trap stiffness and detector sensitivity.

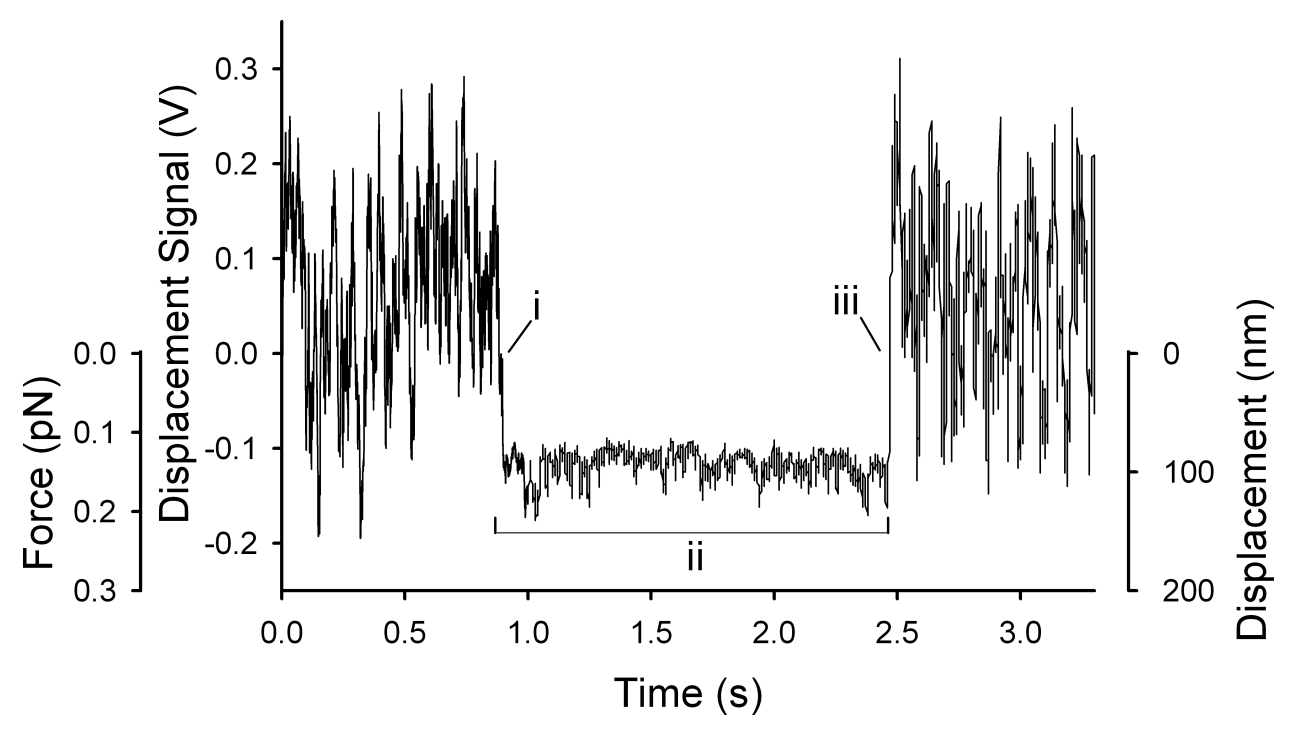

Figure 5: A representative near-zero load data trace collected for an intact HMM-coated target. (i) The actin-coated microsphere forms a bond with the HMM-coated target which is visible as a shift in the displacement signal and a decrease in Brownian motion. (ii) The bond lifetime was measured as the time from when a bond formation was observed, (i), until (iii) the bond ruptured and the microsphere returned to the center of the laser trap. The tensile load experienced by a bond was calculated from the displacement of the signal based upon the stiffness of the trap and the sensitivity of the quadrant photodiode detector. As expected, the noise in the displacement signal is larger than that at higher laser powers. Note the difference in scale of the $y$-axis from Figure 4. The second $y$-axis, Force (pN) is calculated as in Figure 4. Laser trap stiffness is $0.002 \mathrm{pN} / \mathrm{nm}$ for the sample data trace.

Control experiments were performed at $0.145 \mathrm{M} \mathrm{KCl}$ with blank targets (no $\mathrm{HMM}$ ) as well as in the presence of $1 \mathrm{mM}$ pyrophosphate in the microsphere suspension which is thought to be analogous to the prehydrolysis, myosin-ATP state (Gränicher and Portzehl, 1964).

\subsection{Results}

Ionic strength has a modest effect on the actomyosin catch bond

Our data at low (Figure 6a) and physiologic ionic strength (Figure 6b) are consistent with previous reports from our lab that found the actin-HMM bond to behave as a catch-slip 
bond (Guo and Guilford, 2006; Rao et al., 2011). The peak bond lifetimes were greater at physiologic ionic strength compared to low ionic strength, $0.15 \mathrm{~s}$ and $0.11 \mathrm{~s}$, respectively, though this difference is not statistically significant. The critical force (at which peak lifetime occurs) was obtained from the fitted parameters from a two-pathway model (Pereverzev et al., 2005, equation 9) and was similar for the two conditions - 4.09 and 3.95 $\mathrm{pN}$ at low and physiologic ionic strength, respectively. These values are slightly lower than previously reported by us but are well-within the range of forces reported for the isometric force generated by a single myosin molecule (Finer et al., 1994; Guilford et al., 1997; Molloy et al., 1995; Takagi et al., 2006; Tyska et al., 1999), supporting the notion that bond kinetics are closely matched to biomechanical function in myosin (Guo and Guilford, 2006). 

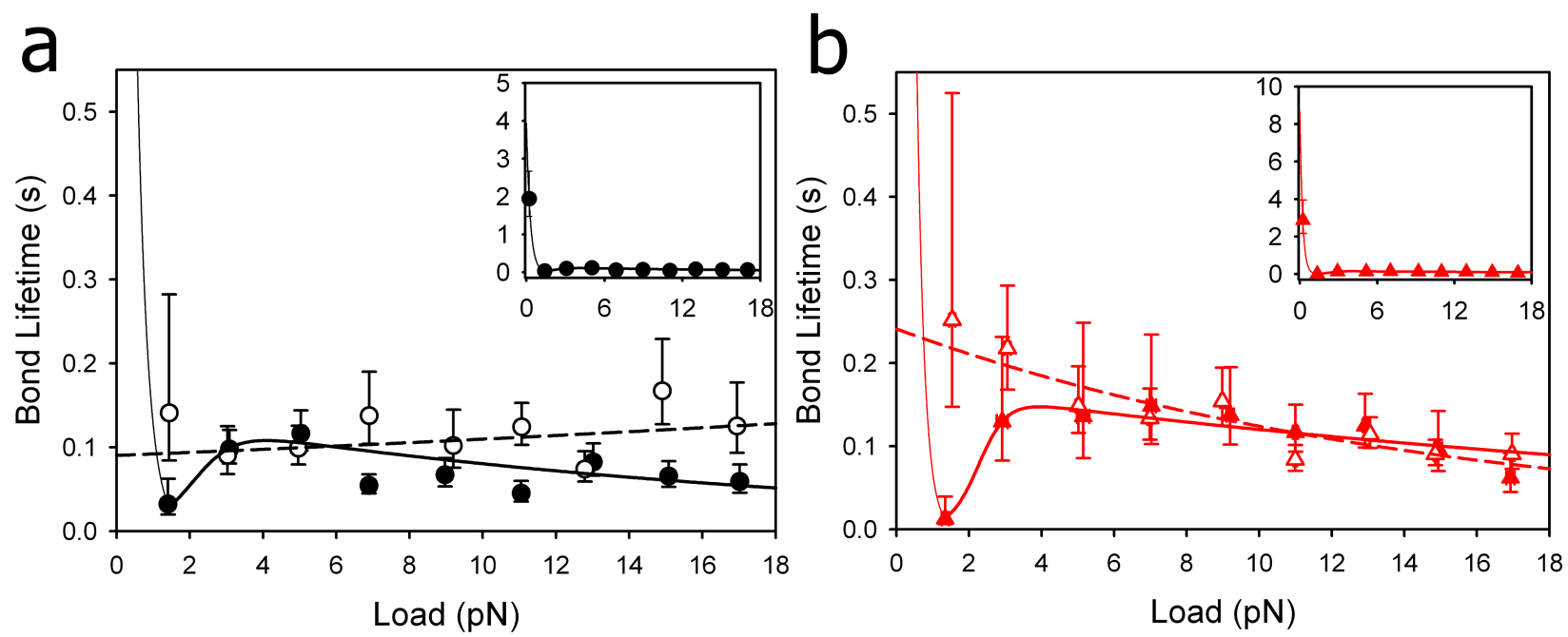

Figure 6: a: At low ionic strength the catch bond formed between intact HMM and actin (circle) is abrogated when loop 2 is cleaved (open circle). Catch bond data were fit by the two-pathway model (bold solid line) (Pereverzev et al., 2005). Cleaved HMM exhibited ideal bond behavior. These data were fit by the single-pathway model (dashed line) (Bell, 1978). Near zero load data at low ionic strength for intact HMM were fit by an exponential decay (unbold solid line) (Guo and Guilford, 2006). Near zero load data points are off the scale of the axis. inset: Same as intact HMM shown in full figure with vertical axis extended to include near zero load data. b: At physiologic ionic strength the catch bond formed between intact HMM and actin (red triangle) is abrogated when loop 2 is cleaved (red open triangle). Lines were fitted as in (a). a \& b: There is a trend toward longer bond lifetimes at physiologic ionic strength though this difference is not statistically significant. Color and symbols are consistent with Figure 8. The mean number of bonds measured per $2 \mathrm{pN}$ force bin was 58 . Horizontal error bars represent the standard error of the mean load for each $2 \mathrm{pN}$ bin and most fall within the symbols. Vertical error bars represent the $95 \%$ confidence intervals of the exponentially distributed data.

As a control, the same measurement was performed at physiologic ionic strength both in the presence of $1 \mathrm{mM} \mathrm{Na}_{4} \mathrm{P}_{2} \mathrm{O}_{7}$ (pyrophosphate; PPi) as well as in the absence of HMM (a blank target surface). Both control conditions resulted in a flat bond lifetime profile over the range of loads measured, confirming that non-specific bonds are roughly loadindependent (Guo and Guilford, 2006; Kong et al., 2009). The binding frequency - the fraction of bonds formed per those attempted - was lower for the control than for the experimental measurements. The binding frequency was 2.8\% and 3.0\% for HMM with PPi and for blank targets, respectively. This compares to $11.1 \%$ and $19.8 \%$ for intact and 
cleaved HMM at low ionic strength, and $18.8 \%$ and $18.5 \%$ for intact and cleaved HMM at physiologic ionic strength. These increased bond frequency and response to load suggest that our bond lifetime measurements are actin-HMM specific.

Solution studies suggest the actomyosin bond lasts for tens to hundreds of seconds in unloaded conditions (Marston, 1982), but more recent data (Guo and Guilford, 2006) as well as the data we present here show a rapid decrease in bond lifetime with infinitesimal loading. The mean bond lifetimes at loads of only $0.21 \pm 0.01 \mathrm{pN}$ for low and physiologic ionic strength were $1.9 \pm 0.6 \mathrm{~s}$ and $2.9 \pm 0.7 \mathrm{~s}$, respectively $(p=0.206)$. Data were fit using an exponential decay, shown in Fig. 3a and b. This divergence of infinitesimal loads from solution studies (zero load) has also been seen in catch bonds unrelated to actomyosin, such as L- and P-selectin with receptor PSGL-1 (Fritz et al., 1998; Marshall et al., 2003; Phan et al., 2006; Sarangapani et al., 2004). It has been postulated that this rapid decrease is due to loading of the actomyosin bond overcoming the tension of water at the interface between the actomyosin molecules in an aqueous environment (Prezhdo and Pereverzev, 2009).

The actomyosin catch bond is the result of allostery Myosin has been proposed to act as an allosteric catch bond (Rao et al., 2011). The allosteric model describes molecules starting in one of two bound states, each with a different dissociation rate, and the ratio of bonds in these states is dependent upon applied force (Thomas et al., 2006). In other words, it is possible that load applied to the actomyosin complex changes the conformation of the complex from a relatively short-lived state to a relatively long-lived state. To test this hypothesis, the fraction of bonds surviving over time was plotted as a function of applied tensile load (Figure 7a) and fitted by exponentials.

As previously reported, the bond survival data was significantly better fit by a double- than a single-exponential decay (Rao et al., 2011); this is suggestive of an allosteric catch bond mechanism (Thomas et al., 2006). A closer look at the force-dependent fraction of bonds surviving in the short- and long-lived states shows that with increasing force the 
proportion of bonds shifts to the long-lived state (Figure 7b). A force-dependent Boltzmann distribution was used to fit the distribution at steady state between the two hypothetical states as in Snook and Guilford (2010),

$$
z_{i}(f) / z_{\text {toral }}(f)=\exp \left(\left(-\Delta E_{i}+f \cdot x_{i \delta}\right) / k_{B} T\right)
$$

where $f$ is applied load, $z$ is the number of bonds in a particular state $i$ (short- or long-lived), $Z_{\text {total }}$ is the total number of bonds, $\Delta E_{i}$ is the energy difference between the two states, $x_{i \delta}$ is the transition state distance between the two states, $k_{B}$ is the Boltzmann constant, and $T$ is the absolute temperature. This assumes that the redistribution between the states reaches rapid equilibrium as force is applied. When the fit was extended to $f=0,91 \%$ of bonds were predicted to be in the short-lived state at zero load (analogous to solution studies, but not reflecting the rapid increase in lifetime $<2 \mathrm{pN}$ ). The fitted value of $\Delta \mathrm{E}=1.8 \pm 0.2 \times 10^{-21} \mathrm{~J}$. The fit also predicts a conformational change of $x_{i \delta}=2.7 \pm 0.5 \AA$ accompanying myosin's transition to the long-lived state. 

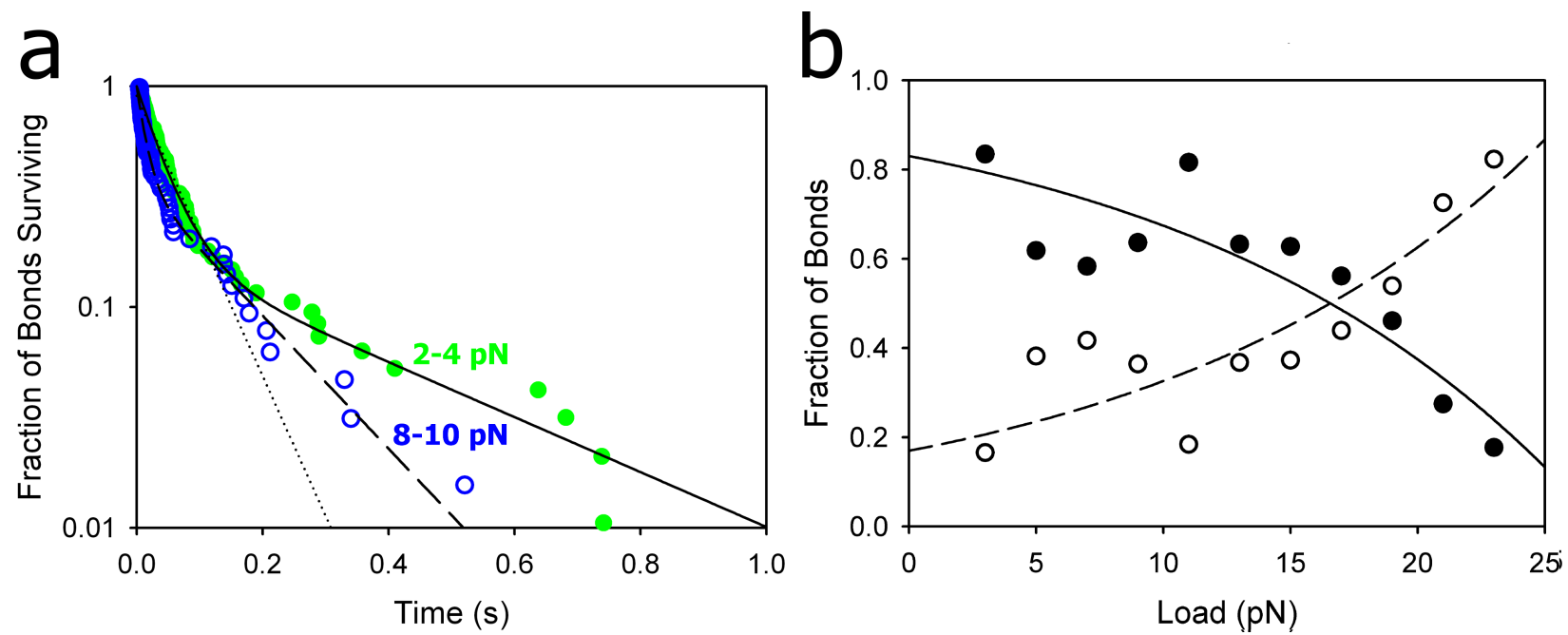

Figure 7: a: The fraction of bonds surviving over time was plotted for two representative force bins, 2$4 \mathrm{pN}$ (green circle, $\mathrm{n}=95$ ) and 8-10 $\mathrm{pN}$ (blue open circle, $\mathrm{n}=63$ ) from the low ionic strength condition with $10 \mu \mathrm{g} / \mathrm{ml}$ HMM present. 2-4 pN data were fit with a single (dotted line) and double exponential decay weighted by time (solid line). 8-10 pN data were fit with a double exponential decay weighted by time (dashed line). The two phases of the double exponential decay suggest a short- and a longlived bond state are present in each population. b: The fraction of bonds in a short- (black circle) and long-lived (black open circle) state for each force bin in the low ionic strength condition with 10 $\mu \mathrm{g} / \mathrm{ml}$ intact HMM is shown. The population of bonds shifts from a short-lived to a long-lived state with increasing tensile load. Data were fit by a force-dependent Boltzmann distribution (Equation 2). The mean total number of bonds in each $2 \mathrm{pN}$ force bin (short- and long-lived state combined) was 56.

Loop 2 is required for catch bond behavior

Catch-bond behavior was abrogated with the cleavage of loop 2, suggesting that an intact actin-binding loop is essential for the force-dependent transition from a short- to a longlived bond state. The bond between actin and cleaved HMM was best fit with a single dissociation pathway from a single bound state (Bell, 1978) at both high and low ionic strength (Figure 6a and b). Interestingly, the surviving bound state is the long-lived state, suggesting that loop 2 functions to weaken the actomyosin bond.

One might argue that the observed differences between intact and cleaved HMM arose from a population of inactive or aberrant heads present in the cleaved HMM. If so, this would 
likely be reflected in a change in actin filament motility driven by cleaved HMM. In vitro motility was performed on intact, cleaved, and fully-digested HMM at equal surface densities in the flow cell. The mean velocities of intact and cleaved HMM were 3.7 \pm 0.07 $\mu \mathrm{m} / \mathrm{s}$ and $4.4 \pm 0.10 \mu \mathrm{m} / \mathrm{s}$ respectively. Actin filaments did not bind to fully digested HMM. The mean velocity of intact and cleaved HMM samples displayed a trend opposite to those reported in Bobkov, et al. (1996), where they observed a small decrease in velocity in cleaved HMM. However, in their study motility of intact HMM was measured under a different experimental condition (no methylcellulose) than was cleaved HMM. Methylcellulose was used for all motility measurements reported here.

\section{Load dependent $2 D$ on-rates}

The load-dependent 2D on-rate (the rate of bond formation) was measured over a range of compressive forces (-4.0-21.7 pN) using a method reported previously by us (Rao et al., 2011) and others (Chen et al., 2008). Intact or cleaved HMM was applied to flow cells at a concentration of $80 \mu \mathrm{g} / \mathrm{ml}$. The surface density was increased because on-rate experiments require a surface density high enough to overcome the limitations of diffusion in the ligandreceptor interaction, whereas bond lifetime measurements necessitate low site densities to ensure single bond events (Snook and Guilford, 2012). An actin-coated microsphere was brought into contact with an HMM-coated target for 1 second, then rapidly stepped $200 \mathrm{~nm}$ away perpendicular to the filament axis for 1 second. This cycle was repeated 30 times.

Bond formation was observed directly in the laser trap. We observe a change in the mean signal magnitude and variance (due to Brownian motion) when the first bond forms between the trapped bead and the target. The time to bond formation $\left(t_{b}\right)$ is the elapsed time between arrival of the bead at the target and the moment of bond formation (Figure 4, position iii). 2D on-rate is inversely related to $t_{b}$ : 


$$
k_{o n}^{0}=\left(t_{b} m_{r} m_{l} A_{c}\right)^{-1}
$$

where $m_{r}$ is the site density of HMM on the nitrocellulose-coated targets (3300 heads $/ \mu \mathrm{m}^{2}$ ) (Guo and Guilford, 2004), $m_{l}$ is the density of available myosin binding sites on actin-coated microspheres ( 637 sites $/ \mu \mathrm{m}^{2}$ ), and $A_{c}$ is the effective contact area between the target and trapped microsphere $\left(0.14 \mu \mathrm{m}^{2}\right)$ as calculated using equations $1 \& 2$ in Rinko et al. (2004). Specific 2D on-rate data were fit using a "reverse Bell model" as in Snook and Guilford (2010). The perpendicular, compressive force $\left(f_{c}\right)$ between the microsphere and the target during bond formation was calculated as the difference between the distance the laser was stepped and the distance the microsphere actually moved after bond rupture, multiplied by the stiffness of the laser trap. Negative $f_{c}$ values indicate no contact between the microsphere and the target, resulting in a time-averaged tensile rather than compressive load.

Load-dependent specific 2D on-rates are shown in Figure 8. With increasing compressive loads the specific 2D on-rates for intact HMM at low ionic strength approached the value reported by Rao et al. $\left(2.0 \times 10^{-4} \mu \mathrm{m}^{2} / \mathrm{s}\right)$ measured under similar conditions but without controlling inward compression (Rao et al., 2011). The two ionic strength conditions displayed similar 2D on-rates at low compressive loads but diverged with increasing compressive load. On-rates were lower at physiologic ionic strength. This inverse relationship between ionic strength and 2D on-rate is consistent with previous measurements of association rates using classical methods (Coates et al., 1985; Marston, 1982; Taylor, 1991; White and Taylor, 1976). 


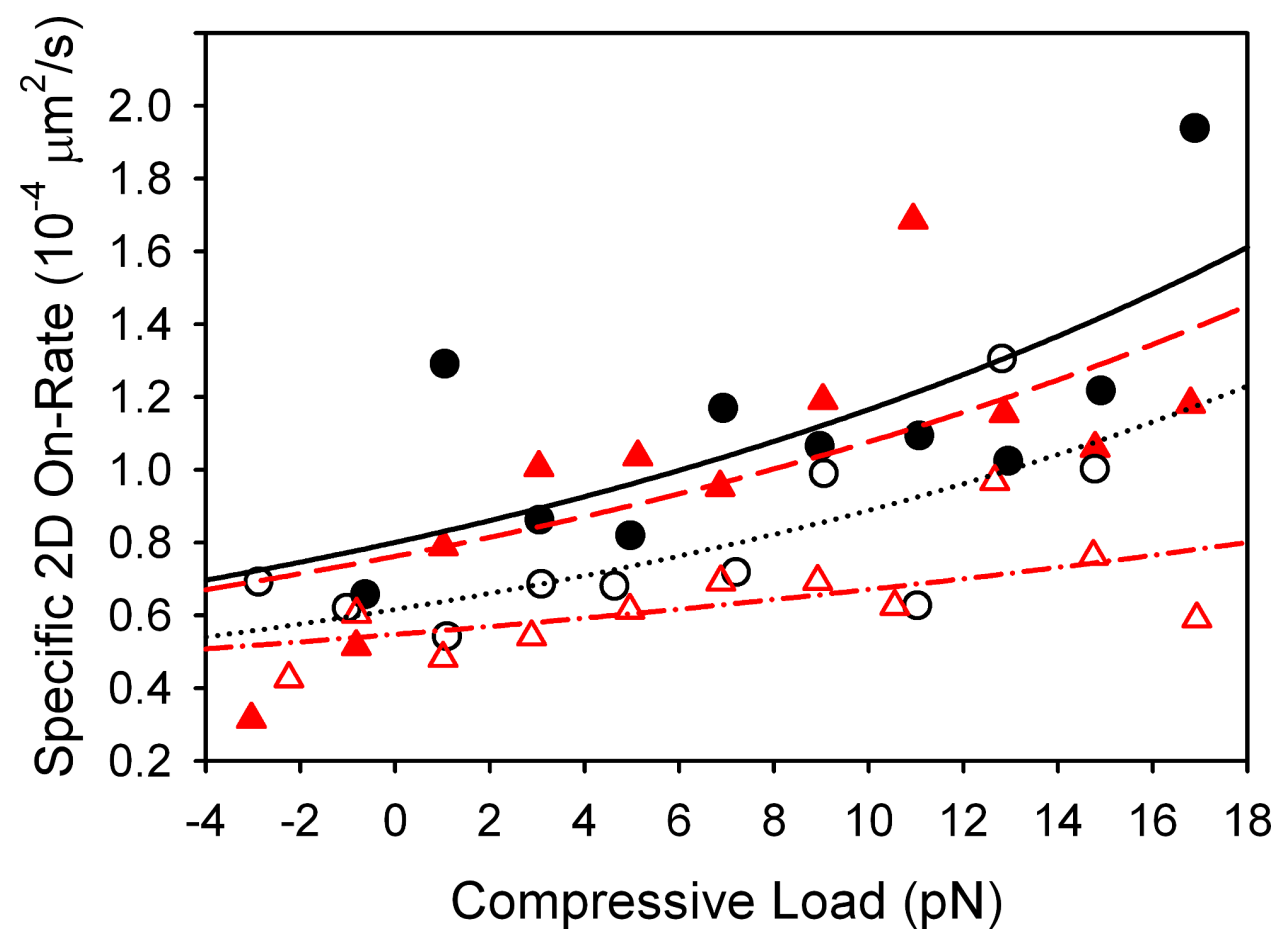

Figure 8: Intact HMM binds to actin faster at low (solid black circle) and physiologic (solid red triangle) ionic strength than cleaved HMM (open black circle and open red triangle are low and physiologic ionic strength, respectively) over a range of compressive loads. The load-dependence of specific 2D on-rate is reduced when cleaved HMM is forming bonds with actin at physiologic ionic strength. Data were fit by a reverse Bell model (Snook and Guilford, 2010). The fitted value for the specific 2D on-rate for cleaved and intact HMM at low and physiologic ionic strength were compared using a $z$ statistic and were not statistically different ( $p=0.179$ and 0.102$)$. The mean number of bonds per $2 \mathrm{pN}$ force bin is 38. The range of independent microspheres used per condition is 139-171. Data were divided into $2 \mathrm{pN}$ bins and data points were plotted at the mean force value for each bin.

HMM with loop 2 cleaved exhibited lower specific 2D on-rates than intact HMM over the range of forces measured. This is particularly apparent for cleaved HMM at physiologic ionic strength, which additionally exhibited reduced load-dependence in its binding rate. Bonds took $\sim 2$ times longer to form at physiologic ionic strength when loop 2 was cleaved $(p=0.102)$. Botts et al. found the actin-S1 association constant decreased when S1 loops 1 and 2 were cleaved (Botts et al., 1982). Bobkov et al. similarly observed decreased affinity of HMM for actin when loops 1 and 2 were tryptically cleaved, and to a lesser extent when only loop 2 was cleaved (Bobkov et al., 1996). Our direct measurements of actin-HMM 
binding confirm that loop 2 cleavage decreases the actomyosin binding rate and show that the reduction is similar at both low and physiologic ionic strength.

\section{Cycling crossbridge model}

If we assume that forces parallel to the filament axis act on the actomyosin crossbridge similarly to those perpendicular to the filament axis we can predict how load-dependent bond rupture may affect the crossbridge attachment time and total cycle time. To this end, we developed a combined biochemical and biomechanical model starting with a population of ADP-bound actomyosin (AMD) and ending with any of the dissociated states - ADPbound myosin (MD), nucleotide-free myosin (M), or ATP-bound myosin (MT) (Figure 9). This model was used to predict the attached-state lifetime as a function of load under physiologic conditions and the attached-state lifetime was subsequently used to calculate a force-velocity relationship. Likewise, the total crossbridge cycle time was modeled from an initial AMD state until the next AMD state was achieved. Biochemical (spontaneous or nucleotide-dependent) and biomechanical (load-dependent) dissociation rates for each of the steps were found in literature or experimentally determined for skeletal actomyosin. Reverse rates were not included in the model.

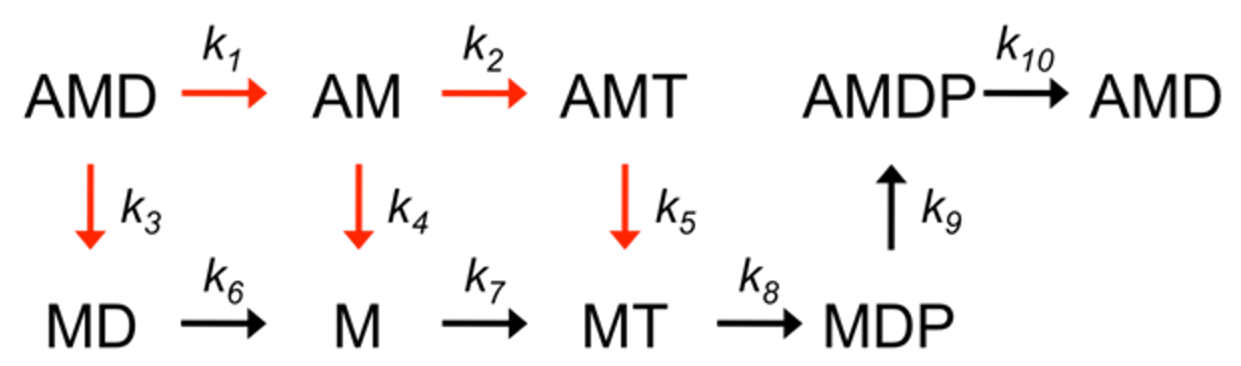

Figure 9: The Cycling Crossbridge Model, with time to dissociation model components in red $\left(k_{1}-k_{5}\right)$ and the full crossbridge cycle in red and black $\left(k_{1}-k_{10}\right)$. In the biomechanical actomyosin unbinding pathway, actin (A) is forcefully dissociated before or after ADP (D) release, and before unbound myosin (M) binds ATP (T). In the biochemical unbinding pathway, ATP binds to actomyosin causing actin dissociation. Biochemical on-rates for skeletal muscle: $k_{2}=5000 \mathrm{~s}^{-1}, k_{3}=0.1 \mathrm{~s}^{-1}$ (Howard, 2001), $k_{4}=1 \mathrm{~s}^{-1}$ (Howard, 2001), $k_{5}=700 \mathrm{~s}^{-1}$ (Millar and Geeves, 1988), $k_{6}=1.85 \mathrm{~s}^{-1}$ (Bagshaw et al., 1973; Trentham et al., 1972), $k_{7}=1000 \mathrm{~s}^{-1}$ (Millar and Geeves, 1988). Biomechanical on-rates for skeletal 


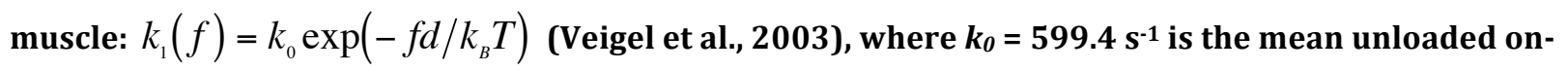
rate for slow and fast skeletal $S 1$ (Capitanio et al., 2006), $f$ is tensile force, and $k_{B} T$ is thermal energy. The crossbridge conformational change distance parameter associated with ADP release (also known as "the second power stroke) is accepted to be small; we assumed it to be $d=0.5 \mathrm{~nm}$. The remaining two on-rates are both represented by the same one- or two-pathway model. For the catch bond twopathway model of bond rupture described earlier to fit our experimental data: $k_{3}=k_{4}=k_{c}^{0} \exp \left(x_{c} f / k_{B} T\right)+k_{s}^{0} \exp \left(x_{s} f / k_{B} T\right)$ (Pereverzev et al., 2005), where $\boldsymbol{k}_{c}=312.5 \mathbf{s}^{-1}$ is the unloaded on-rate for the catch pathway, $x_{c}=-\mathbf{- 7 . 2} \mathrm{nm}$ is the characteristic bond length for the catch pathway, $k_{s}=7.1 \mathrm{~s}^{-1}$ is the unloaded on-rate for the slip pathway, and $x_{s}=0.2331 \mathrm{~nm}$ is the characteristic bond length for the slip pathway. For the slip bond single-pathway model of bond

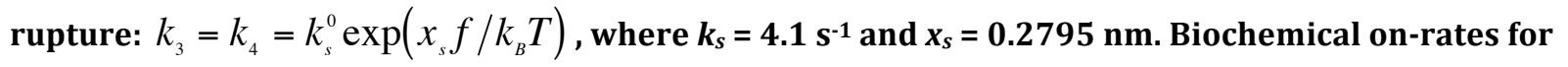
phasic smooth muscle were as follows: $k_{2}$ and $k_{5}$ have a combined rate $=380 \mathrm{~s}^{-1}$ (Khromov et al., 1996), $k_{3}=0.0035 \mathrm{~s}^{-1}, k_{4}=0.004 \mathrm{~s}^{-1}, k_{6}=1.9 \mathrm{~s}^{-1}, k_{7}=12.8 \mathrm{~s}^{-1}$ (Cremo and Geeves, 1998). Biomechanical on-rate for smooth muscle: $k_{1}$ is the same as above with $k_{0}=32 \mathrm{~s}^{-1}$ and $d=2.7 \mathrm{~nm}$ (Veigel et al., 2003). Smooth muscle was additionally modeled with biomechanical actomyosin unbinding using the experimental $k_{3}$ and $k_{4}$ from skeletal muscle for both the catch and slip bond because these values are unavailable for smooth muscle. Initial unbound ATP concentration is $4 \mathrm{mM}$. Initial AMD concentration is $4.18 \mu \mathrm{M}$ for skeletal muscle and $0.352 \mu \mathrm{M}$ for smooth muscle, estimated from number of myosin heads present (Herrera et al., 2002; Linari et al., 1998) and duty ratio (Guilford et al., 1997) per sarcomere or cell volume (Guilford and Warshaw, 1998; Linari et al., 1998).

There is evidence ADP dissociation from actomyosin is a function of load $\left(k_{1}(f)\right)$, where the rate of dissociation is estimated as:

$$
k_{1}(f)=k_{0} \exp \left(-f d / k_{B} T\right)
$$

where $k_{0}$ is the detachment rate at zero load, $f$ is the applied load, $d$ is the crossbridge conformational change distance parameter, and $k_{B} T$ is thermal energy (Veigel et al., 2003).

The results of the current work as well as others have shown that actin dissociation from both ADP-bound and rigor actomyosin (Guo and Guilford, 2006; Rao et al., 2011) are likewise functions of load by a two-pathway (catch bond) model: 


$$
1 / t_{c s}(f)=k_{c}^{0} \exp \left(x_{c} f / k_{B} T\right)+k_{s}^{0} \exp \left(x_{s} f / k_{B} T\right)
$$

where $t_{c s}(f)$ is the mean bond lifetime at force $f$ for a catch bond, $k_{c}{ }^{o}$ and $k_{s}{ }^{o}$ are the dissociation constants for unbinding through the catch and slip pathways, respectively, $x_{c}$ and $x_{s}$ are the characteristic bond lengths of the catch and slip pathways, respectively (Pereverzev et al., 2005). This representation of catch-slip behavior is reasonable when the transition between the short-lived and long-lived states is fast. Experimental values of smooth muscle biomechanical dissociation for the two-pathway model are unavailable so the experimentally determined skeletal muscle parameters from this study were used in the phasic smooth muscle model.

A single pathway (slip bond) model was also used to model actomyosin dissociation as a function of load to determine the importance of catch bond behavior:

$$
1 / t_{s}(f)=k_{s}^{0} \exp \left(x_{s} f / k_{B} T\right)
$$

where $t_{s}(f)$ is the mean bond lifetime at force $f$ for a slip bond.

Velocity was calculated for each of the dissociation models (biochemical, slip biomechanical, and catch biomechanical) by dividing the time to $1 / 2$ detachment by myosin's unitary step size to predict the skeletal and smooth muscle force-velocity relationship. The unitary step size is $5 \mathrm{~nm}$ for skeletal (Finer et al., 1994; Guilford et al., 1997; Kitamura et al., 1999; Saito et al., 1994; Uyeda et al., 1990) and smooth muscle myosin (Guilford et al., 1997).

\section{The catch bond increases the crossbridge dissociation rate}

The cycling crossbridge model predicts that the catch bond of skeletal muscle myosin may accelerate unloaded shortening at forces below $2 \mathrm{pN}$ of tensile load compared to the biochemical model (Figure 10). The time to crossbridge dissociation is similar for both biochemical and catch or slip biomechanical actomyosin dissociation over a range of forces 
above $2 \mathrm{pN}$, but is shortened below $2 \mathrm{pN}$ when the catch bond is included. Therefore, the majority of crossbridges will dissociate due to ATP binding at forces above $2 \mathrm{pN}$, and due to bond rupture below $2 \mathrm{pN}$. The model similarly predicts that the skeletal muscle duty ratio will be reduced at loads less than $2 \mathrm{pN}$ (Figure 11) compared to biochemical dissociation, suggesting that loop 2 increases the unloaded sliding velocity at the expense of force generation at low loads. The duty ratio calculated by the model is similar to the experimentally-determined 0.71-3.8\% reported previously (Guo and Guilford, 2004; Harris and Warshaw, 1993).

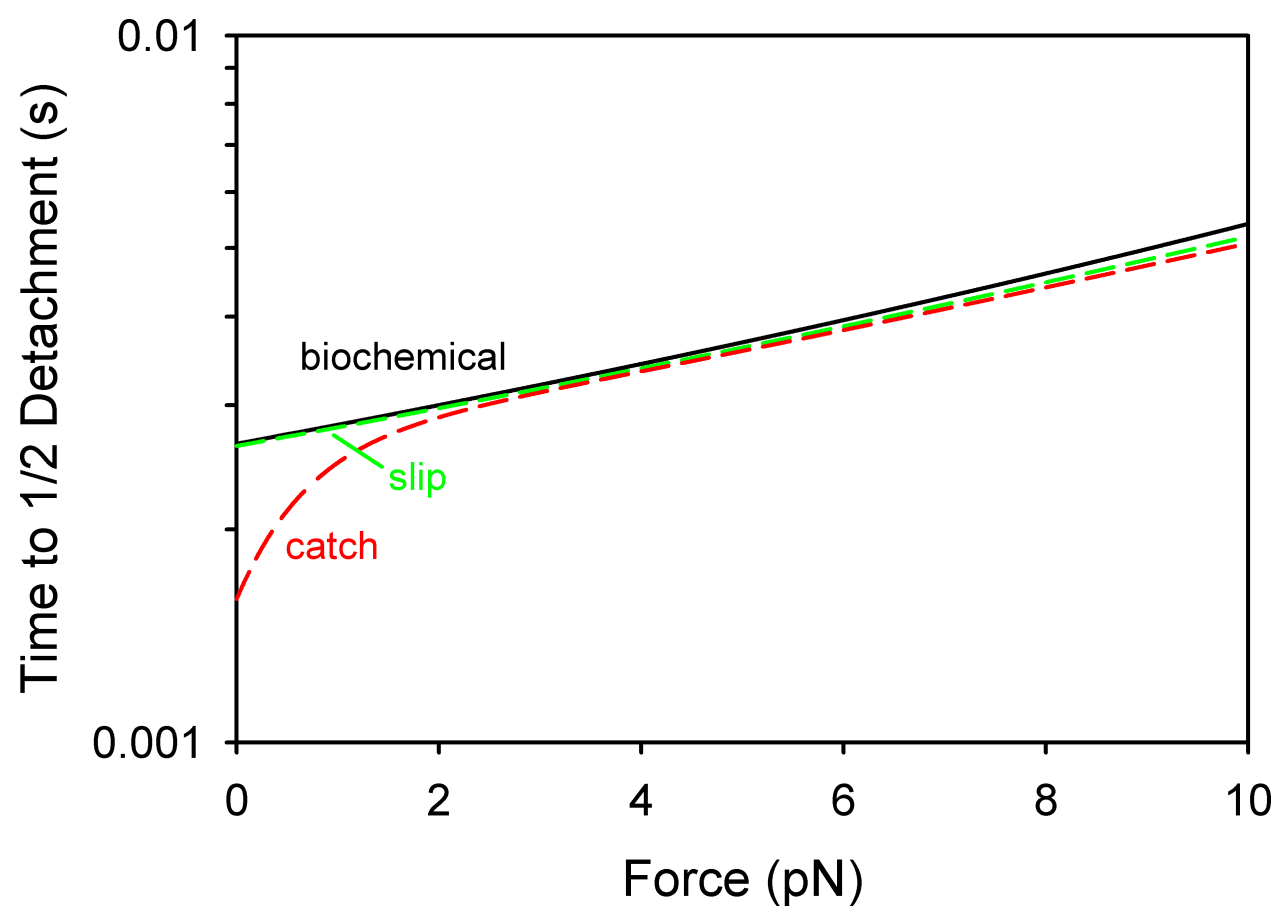

Figure 10: The time for half of the skeletal muscle crossbridge population to reach an unbound state, determined by the pathway presented in Figure 9 over a range of forces with biochemical (solid line), slip bond biomechanical (green dashed line), or catch bond biomechanical (red dashed line) actomyosin dissociation in steps 3 and 4 . Catch bond biomechanical crossbridge dissociation is predicted to cause faster crossbridge detachment and therefore shorter bond lifetimes than biochemical dissociation alone or slip bond biomechanical dissociation at tensile loads $<2 \mathrm{pN}$. The biomechanical and biochemical crossbridge dissociation rates are predicted to be similar at forces $>2$ pN for skeletal muscle. 


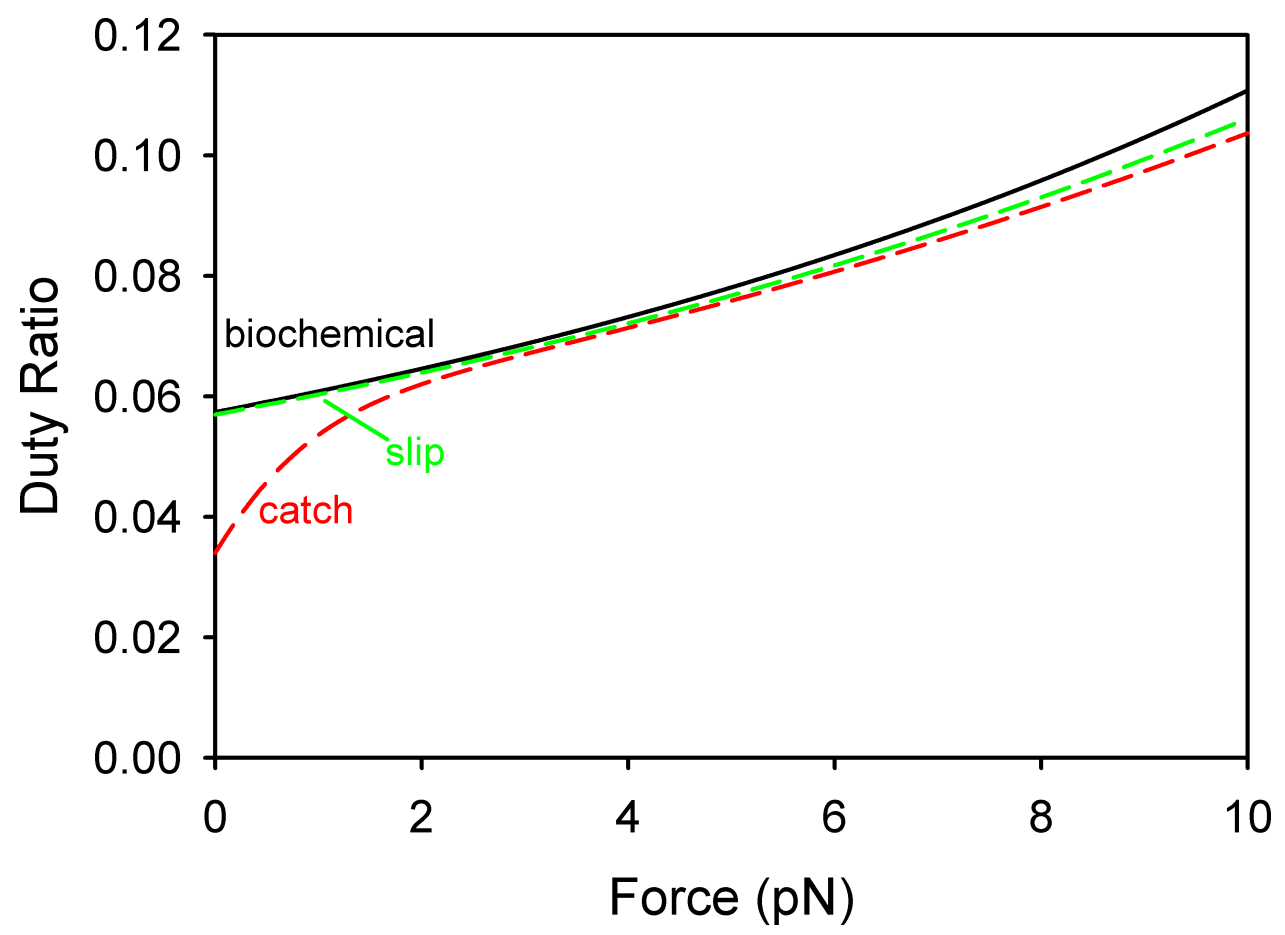

Figure 11: The duty ratio of skeletal muscle crossbridges predicted by the cycling crossbridge model over a range of forces with biochemical (solid line), slip bond biomechanical (green dashed line), or catch bond biomechanical (red dashed line) actomyosin dissociation in steps 3 and 4 of the model. Duty ratio is reduced by catch bond biomechanical dissociation at forces below $2 \mathrm{pN}$.

But where the catch bond may have a dramatic impact is in a slower myosin, like smooth muscle myosin.

If we apply our skeletal actomyosin catch bond dissociation rates to a model of phasic smooth muscle myosin, the force-dependent catch bond may significantly decrease the actomyosin bond lifetime and duty ratio over a broad range of tensile loads. In a slower muscle type such as phasic smooth muscle, the force-dependent catch bond may significantly decrease the actomyosin bond lifetime (Figure 12) and duty ratio (Figure 13) over a broad range of tensile loads. When the actomyosin catch bond is included in the model crossbridges dissociate more quickly than when steps 3 and 4 are mediated by biochemical dissociation alone. As expected, the time to detachment in the slip bond biomechanical model was similar to the biochemical time at low forces and diverged with increasing tensile load, resulting in decreased time to detachment to a value similar to that 
of the catch bond biomechanical model. The smooth muscle duty ratio for the biochemical model increases with force to a value close to $1(100 \%)$. Biomechanical dissociation significantly reduces the duty ratio, with slip bond biomechanical values diverging with increasing force from those of biochemical dissociation.

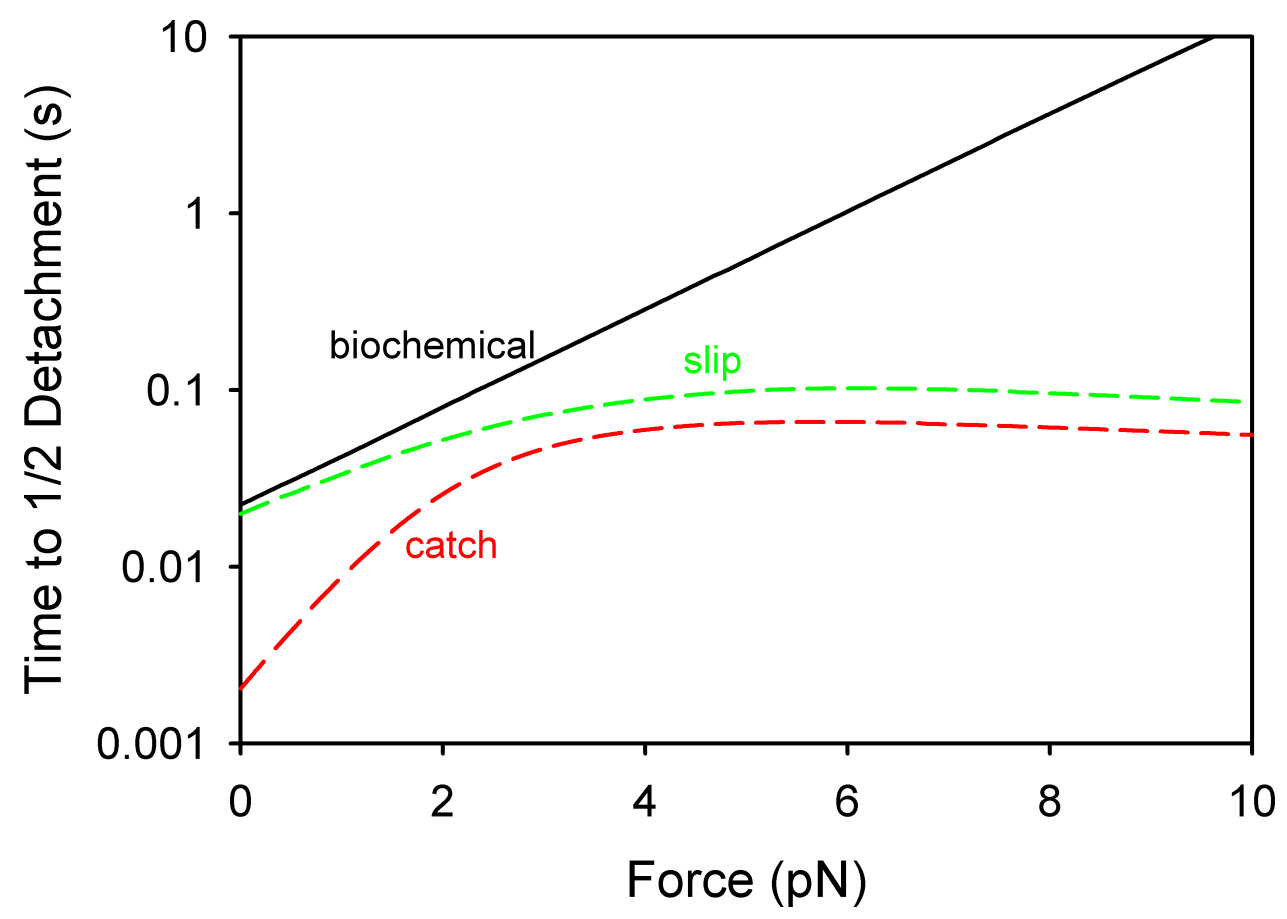

Figure 12: The time for half of the smooth muscle crossbridge population to reach an unbound state, determined by the pathway presented in Figure 9 over a range of forces with biochemical (black solid line), slip bond biomechanical (green dashed line), and catch bond biomechanical (red dashed line) actomyosin dissociation in steps 3 and 4 . Note the difference in scale of the y-axis from Figure 10. Biomechanical crossbridge dissociation is predicted to result in faster crossbridge dissociation and therefore shorter bond lifetimes than by biochemical dissociation alone. 


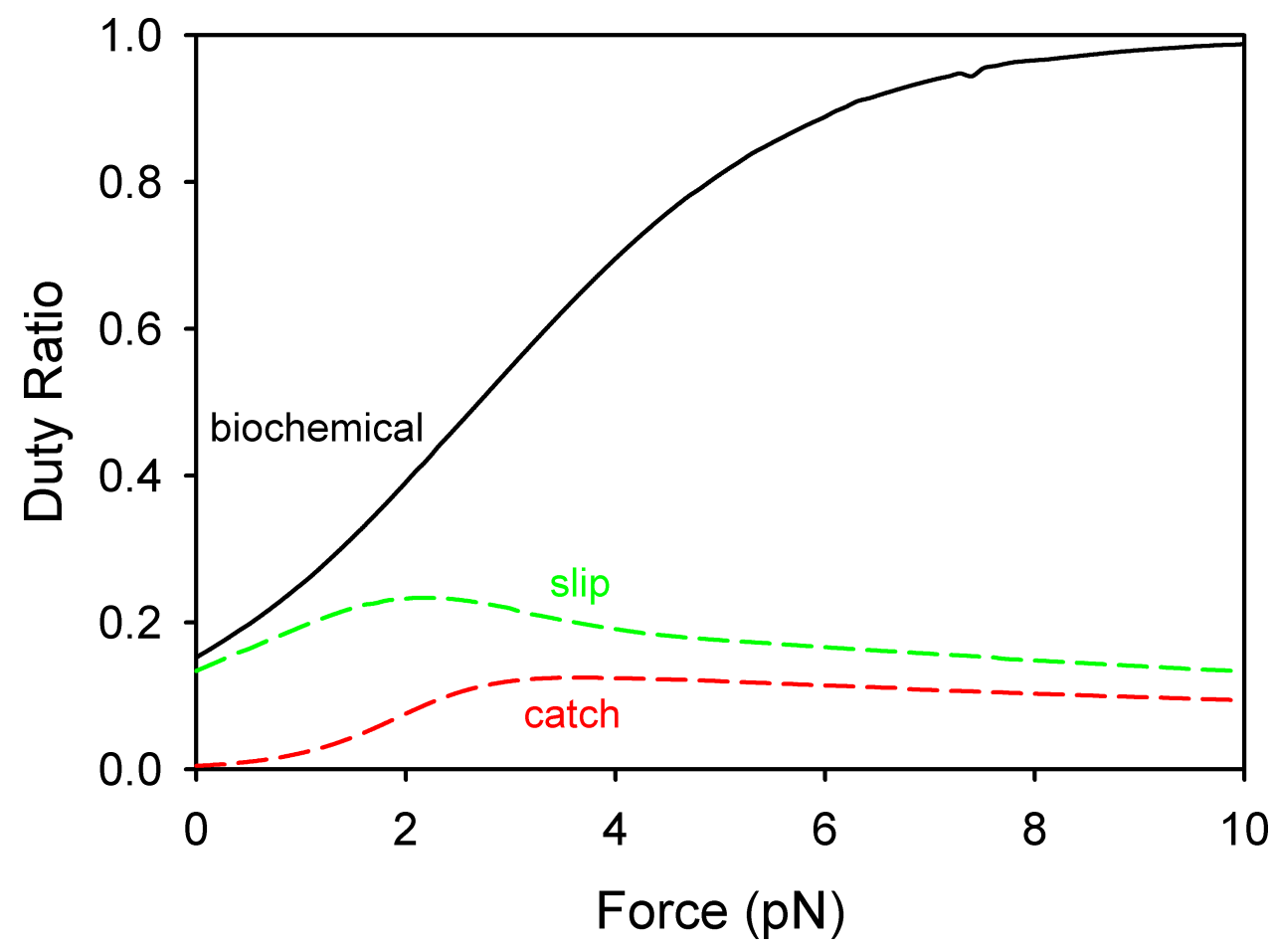

Figure 13: The duty ratio of smooth muscle crossbridges predicted by the cycling crossbridge model over a range of forces with biochemical (black solid line), slip bond biomechanical (green dashed line), or catch bond biomechanical (red dashed line) actomyosin dissociation in steps 3 and 4 of the model. Duty ratio increases with force for biochemical dissociation and is significantly reduced for slip bond biomechanical dissociation with increasing force. Catch bond biomechanical dissociation significantly reduces duty ratio over the range of forces.

The time to crossbridge dissociation was used to predict a force-velocity relationship for skeletal and smooth muscle. Median velocity should be proportional to the inverse time to $1 / 2$ detachment. Interestingly, Figure 14 suggests that a catch bond is required for a single molecule to exhibit a hyperbolic force-velocity relationship similar to that originally measured by Hill (1938) and classically representative of muscle fiber force-velocity measurements. The same behavior has also been observed in mini-ensemble measurements between a single actin filament and $\sim 8$ myosin heads, showing that this relationship holds true even when scaled down to the molecular level (Debold et al., 2005). This hyperbolic relationship is muted in smooth muscle or absent in skeletal muscle for biochemical or slip biomechanical actomyosin dissociation. 

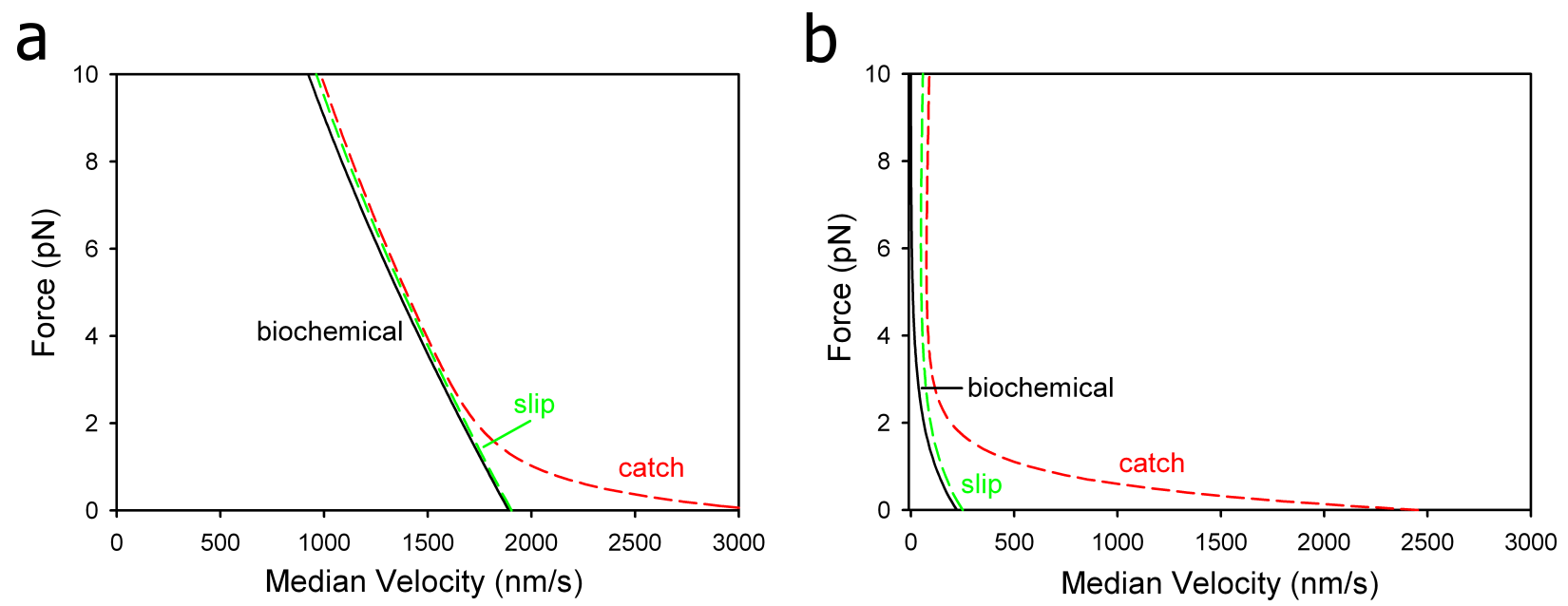

Figure 14: The force-velocity relationship predicted by the cycling crossbridge model for biochemical (solid line), biomechanical slip (green dashed line), and biomechanical catch (red dashed line) actomyosin dissociation in steps 3 and 4 for a: skeletal and b: smooth muscle. Median velocity is the inverse of the time to $1 / 2$ detachment.

Our cycling crossbridge model was designed for measuring the time between different states of myosin and not meant to describe aggregate behaviors of myosin in an assembly, but the model does suggest how it might behave. The failure of this model to predict $F_{0}$, the force at which velocity drops to zero, is addressed in the discussion.

\subsection{Discussion}

Our data confirm that loop 2 accelerates actin-myosin binding, but also reveal that loop 2 serves as a force-dependent inhibitor of a long-lived rigor state.

Catch-bond behavior arises from the ability of actomyosin to form a short- or long-lived bound state with the balance between them being force-dependent. One might suppose that these two bound states are the weakly and strongly bound states; however, catch bond behavior remained when the ionic strength was increased almost six fold. Therefore, it is independent of weak binding, and the short- and long-lived states comprising the actomyosin catch bond must arise from two force-dependent strong-binding states of myosin. As our experiments were performed in the absence of nucleotide, these data suggest that myosin has two rigor states, and that force regulates the distribution between 
them via loop 2. The actomyosin bond will typically form in the short-lived state, and rupture from either the short- or long-lived states as biased by internally and externally applied loads.

Rao et al. and others have suggested that myosin acts as an allosteric catch bond resulting from movement at one of its hinge regions (Rao et al., 2011; Thomas et al., 2008). The present data, however, suggest that a much smaller change in loop 2 or its adjoining domains is responsible for the allosteric change from a short- to a long-lived bond state shown in the scheme in Figure 15. Removal of either pathway would result in slip-bond behavior, but the bond lifetime at low forces reveals which pathway has been eliminated. Indeed, when loop 2 was cleaved catch bond behavior was eliminated and only the longlived pathway remained. Figure $6 \mathrm{a}$ and $\mathrm{b}$ show that loop 2 cleavage extended bond lifetimes at low forces to durations greater than at the critical force of the catch bond; this is the long-lived pathway. 


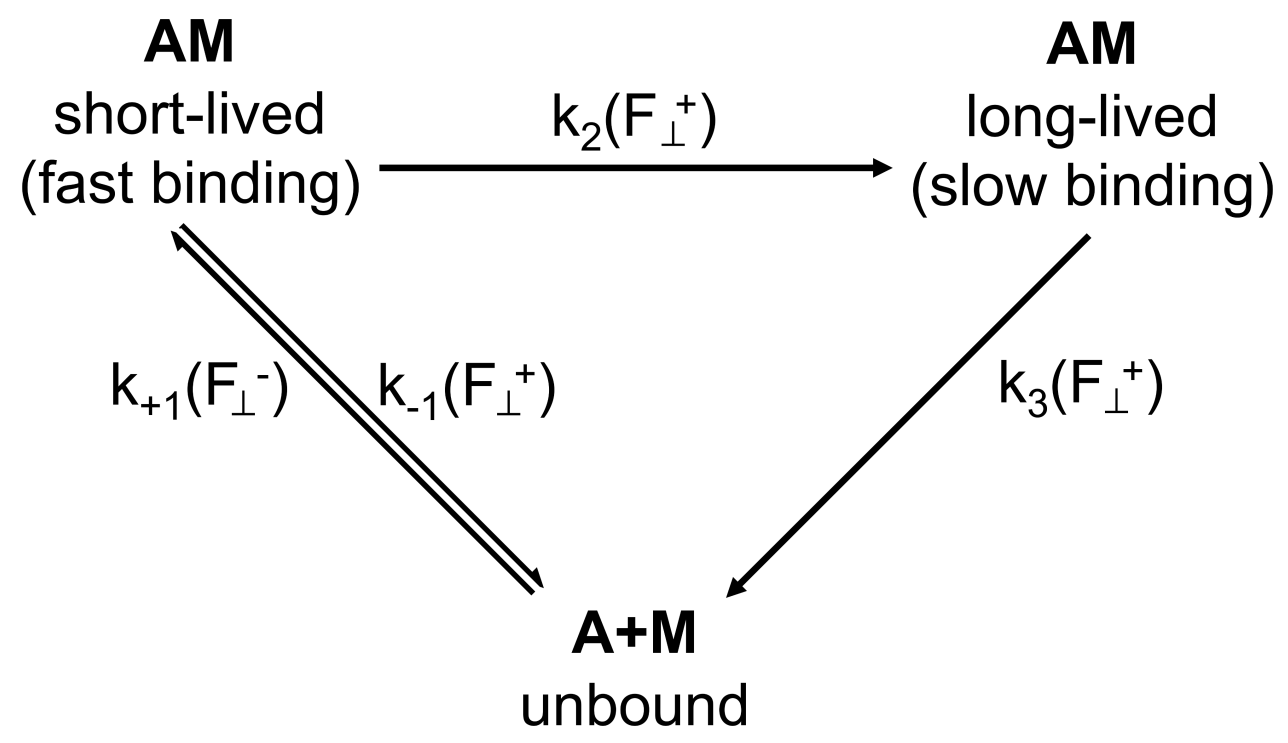

Figure 15: A scheme of the two states in which rigor myosin can bind actin (AM) and unbind (A+M) from actin. Myosin binds at rate $k_{+1}\left(F_{i}\right)$ in the short-lived state under a compressive load $F_{-}^{-}$applied perpendicular to the filament axis. A perpendicular tensile load $F_{+}{ }^{+}$applied to this short-lived bond will cause the bond either to break at rate $k_{-1}\left(F_{+}^{+}\right)$or to transition into a long-lived bond state with rate $k_{2}\left(F_{+}^{+}\right)$. Bonds that are in a long-lived bond state rupture at rate $k_{3}\left(F_{+}^{+}\right)$. Bonds form and rupture faster in the short-lived state than in the long-lived state. The majority of bonds form in the short-lived state and transition to a long-lived state instead of forming directly in the long-lived bond state.

The rates of actomyosin bond rupture were experimentally determined to be loaddependent. Our experiments, however, were performed in a single nucleotide state (rigor). We therefore sought to determine the overall effect of load-dependent actomyosin dissociation on the crossbridge cycle. To understand how the load-dependent rupture of the actomyosin bond could potentially affect a cycling crossbridge, and the role of loop 2 in particular, we modeled rate of passage of myosin through the crossbridge cycle under the influence of load. Our model predicts the time required for a population of actomyosin bonds starting in the ADP-bound state to dissociate through biochemical (spontaneous or nucleotide-driven dissociation) or biomechanical (load-dependent dissociation) pathways to unbound myosin. Interestingly, our model predicts that skeletal muscle crossbridges are more likely to dissociate because of applied mechanical tension at low loads than by nucleotide binding. The opposite is true above $\sim 2 \mathrm{pN}$. Therefore, the main impact of the catch bond on the cycling crossbridge in skeletal muscle may be to enable higher unloaded 
shortening velocities. During shortening, attached crossbridges impose a resistive drag on others that are undergoing power strokes, thus reducing sliding velocity. Hooft et al. suggested that reducing the bond duration of resistive myosin heads increases the shortening velocity (Hooft et al., 2007), thereby overtaking ATP binding in limiting shortening velocity. When a similar model is applied to a slower-cycling phasic smooth muscle, however, the catch bond is predicted to increase the crossbridge dissociation rate and therefore decrease bond duration over a wide range of forces (0-10 pN). This may act as a mechanism to prevent crossbridges from remaining bound too long. The evolutionary history of class II myosin (Korn, 2000) suggests that the loop 2 catch bond function may have developed in smooth and non-muscle myosin, but may have later become vestigial in skeletal muscle myosin where the crossbridge movement accompanying ADP release is small.

The reader is cautioned, however, that not all the necessary parameters are available for an accurate and comprehensive model of phasic smooth muscle. These unknowns include the biomechanical dissociation rates of smooth actomyosin, and the rate of phosphate release from myosin in step 9. As a result, our estimates of detachment rate and duty ratio differ significantly from published estimates, particularly for the catch bond biomechanical model (Harris and Warshaw, 1993; Veigel et al., 2003). However, we know there is a steep rise in bond lifetime at zero load that could explain this difference (Guo and Guilford, 2006). Nonetheless, we predict that the catch bond will result in a dramatic decrease in duty ratio, providing evidence that loop 2 is an important regulator of bond lifetime that may prove especially important in slower muscles.

The force-velocity relationships predicted by our simple model reflect the inherent loaddependence of the actomyosin interaction at the single-molecule level and shows that it may be due to catch bond biomechanical dissociation, not just the load dependence of biochemical kinetics alone. However, the cycling crossbridge model we present was not meant for calculating force-velocity curves and has hidden assumptions that are not conducive to a proper force-velocity prediction. For example, our model does not take into account to the compliance of the molecules, which would lead to futile motion, nor does it 
incorporate the load-dependence of the power stroke or the drag caused by neighboring crossbridges. Further, our model relies upon biomechanical dissociation rates that we measured with tension applied perpendicular to the axis of the filament. This loading direction is less physiological than axial loading, but offers a defined direction to the load on the bond. The compliance of the myosin molecule suggests that a tension applied to the actomyosin crossbridge parallel to the filament axis is not pure shear, nor is it purely tensile or compressive.

Though allostery accounts for catch bond behavior in bond rupture, the allosteric model of bond formation does not account for the observed load-dependence of bond formation (Snook and Guilford, 2010). Our load-dependent specific 2D on-rate data suggest that in applying a compressive load we are forcing the opposing binding sites together. This has been termed a "reverse Bell model," in which the energy landscape for bond formation is tilted and therefore activation energy required to form a bond is lowered (Snook and Guilford, 2010).

There is not enough evidence to determine whether the observed effects of compressive load on actomyosin binding are physiologically important. As the filaments slide, the bond formation may occur under a compressive force if it does so as the myosin head is approaching its binding site on actin; this is because the continuing motion of the filaments may drive the myosin head into its binding site. Similarly, bond formation may occur under a tensile force if it does so after the myosin head has passed its binding site on actin.

Ionic strength is an important factor in the initial collision complex formation between actin and myosin as well as development of the subsequent attached state in actomyosin binding (Geeves and Conibear, 1995). Because of decreased actin-myosin binding affinity at physiologic ionic strength, classical solution studies (Eisenberg and Moos, 1968; White and Taylor, 1976) and actin-myosin in vitro motility (Takiguchi et al., 1990) have often been performed in non-physiologic, low ionic strength conditions. The geometric constraints acting on actin and myosin in vivo overcomes the problem of decreased binding affinity. The design of our experiments creates a partially constrained geometry in which the 
molecules are restricted to approximately planar movement, thereby allowing the actomyosin bond to be examined at physiologic ionic strength.

The data in Figure $6 a$ and $b$, and Figure 8 show mirroring effects of ionic strength on bond formation and rupture. That is, physiologic ionic strength gives rise to low rates of bond formation but also gives rise to low rates of bond rupture (i.e. long lifetimes). Similarly, reduced ionic strength gives rise to high rates of bond rupture and also high rates of bond formation. Microscopic reversibility explains this as the same energy barrier being overcome to break a bond as was overcome in forming it. In this instance, ions must be excluded from the binding interface to allow ionic and hydrophobic interactions to form. When the ion concentration is increased, more energy is required to remove the ions from the binding interface. Similarly, there is an increase in energy needed to rescind the hydrophobic interactions between the binding interfaces of the two proteins and to resolvate them when ionic strength is high.

\subsection{Conclusion}

Loop 2 was previously thought to act only in searching out the myosin binding site on actin. Our data suggest that loop 2 also regulates actomyosin crossbridge kinetics in a forcedependent manner through its integral role in catch bond behavior, supporting the hypotheses we put forward in Aim 1A and B. This introduces loop 2 as an important regulator of the actomyosin rigor bond. We were also able to make simple predictions of the effect of loop 2 load-dependent actomyosin unbinding on crossbridge cycling using the model proposed as part of Aim 1C. 


\section{Chapter 3: cMyBP-C \\ phosphorylation-dependent crossbridge regulation}




\subsection{Introduction}

cMyBP-C is a regulatory protein that modulates muscle structure and function in the heart. This $140 \mathrm{kDa}$ protein is made up of $11 \mathrm{immunoglobulin}$ and fibronectin type III domains. The C-terminus of cMyBP-C is thought to anchor it to the thick filament backbone and titin, and the $\mathrm{N}$-terminus is thought to be responsible for regulating crossbridge kinetics. It has been proposed that the $\mathrm{N}$-terminal domains of cMyBP-C bind physiologically both to actin and to myosin (Pfuhl and Gautel, 2012). Recent evidence suggests that cMyBP-C also interacts directly with tropomyosin to promote thin filament activation (Mun et al., 2014). The cardiac isoform is of particular interest as mutations in cMyBP-C are a leading cause of hypertrophic cardiomyopathy (Harris et al., 2011), and phosphorylation is implicated in modulating the ability of cMyBP-C to regulate the actomyosin crossbridge (Sadayappan et al., 2005, 2006). cMyBP-C phosphorylation also appears to protect tissue from ischemiareperfusion injury, suggesting that $\mathrm{CMyBP}-\mathrm{C}$ may be cardioprotective under some conditions.

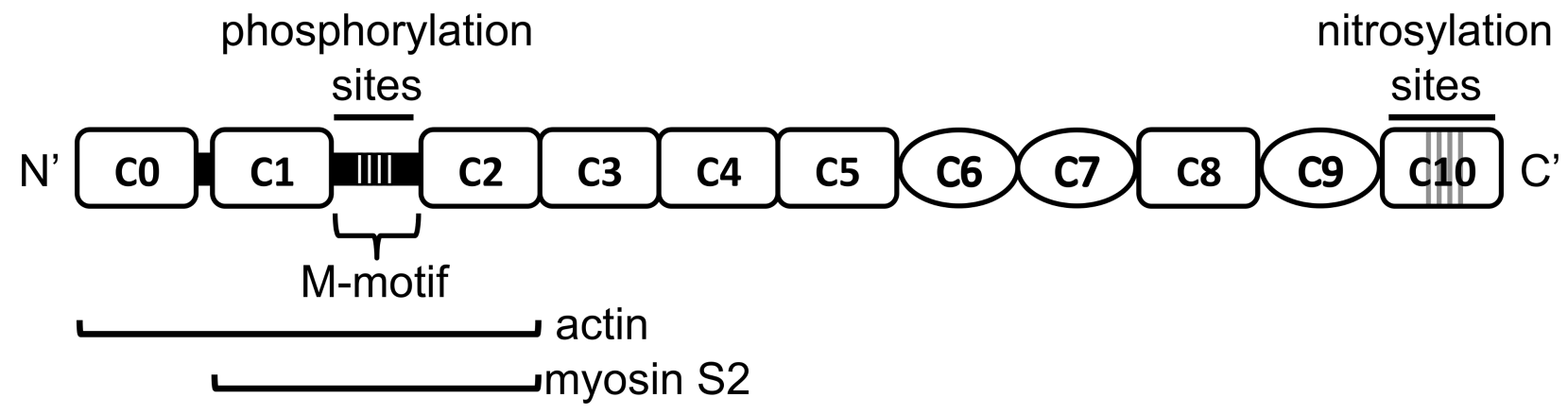

Figure 16: Diagram of cMyBP-C domains where immunoglobulin-like domains are rectangular and fibronectin type III domains are oval. The disordered M-motif between domains $\mathrm{C} 1$ and $\mathrm{C} 2$ contains 4 phosphorylation sites. Domain C10 contains 4 nitrosylation sites. Domains evidenced to bind actin and myosin $S 2$ are indicated.

All isoforms of MyBP-C (skeletal and cardiac) contain a disordered region known as the Mmotif, which lies between domains $\mathrm{C} 1$ and $\mathrm{C} 2$. The cardiac isoform contains four putative regulatory phosphorylation sites responsive to $\beta$-adrenergic stimulation, serines S273, S282, S302, and S307 (in mice) (Gautel et al., 1995; Jia et al., 2010). Phosphorylation is 
hierarchical, starting with S282, which then allows phosphorylation of S273 and S302 (Gautel et al., 1995). Substantial cMyBP-C phosphorylation has been observed in humans and mice (Copeland et al., 2010; Dijk et al., 2009; Jacques et al., 2008; Sadayappan et al., 2005, 2006), and its dephosphorylation is characteristic of heart failure (Copeland et al., 2010; Jacques et al., 2008; Sadayappan et al., 2005). cMyBP-C phosphorylation has been shown to regulate crossbridge function in a graded manner (Weith et al., 2012b).

The N-terminal C0-C1 domains and first 17 amino acids of the M-motif of cMyBP-C are minimally required for phosphorylation-modulated inhibition of in vitro actomyosin motility (Weith et al., 2012a), but it remains unclear which crossbridge protein is the primary physiologic binding partner of cMyBP-C.

Here we use single-molecule force spectroscopy to measure the bond lifetimes of Nterminal cMyBP-C with actin and with HMM in the rigor state over a range of applied tensile loads. We performed these measurements using a fragment of cMyBP-C that includes domains $\mathrm{C} 0$ through $\mathrm{C} 3$, a region encompassing the reported actin and myosin binding domains as well as the phosphoregulatory M-motif. To control the phosphorylation state of the M-motif, two bacterially-expressed fragments were used - one with native serine residues and a second in which the four endogenously phosphorylatable serines were replaced with aspartic acid to mimic the phosphorylated state. Our data suggest that $\mathrm{N}$-terminal cMyBP-C does not regulate crossbridge force generation through imparting a phosphorylation-dependent drag on the actin filament or the myosin head alone. We propose that $\mathrm{cMyBP}-\mathrm{C}$ requires the actomyosin complex as a binding partner to regulate cardiac contractility through an imposed mechanical drag or through alteration of a kinetic rate of the crossbridge cycle.

\subsection{Methods}

\section{Proteins}

HMM and F-actin were purified from rat skeletal muscle as described in Appendix 1, Section A1.1. For laser trap experiments, F-actin was biotinylated and coupled to 
streptavidin-coated microspheres. For use in the in vitro motility assay, F-actin was stabilized with TRITC-phalloidin.

cMyBP-C N-terminal fragments were provided by the Warshaw Lab (University of Vermont). These were bacterially expressed from mouse cardiac cDNA using a pET expression system (Novagen, Madison, WI) (Sadayappan et al., 2005). Two different Nterminal fragments were expressed, wild type C0C3 (amino acids 1-539) and C0C3 with aspartic acids (D) to mimic phosphorylation at S273, S282, S302, and S307 (referred to as C0C3-4D). Fragments used in HMM bond lifetime experiments were expressed containing a biotin attached to the $\mathrm{N}$-terminus of domain $\mathrm{C} 0$ by a 15 amino acid peptide tag (Avidity, LLC, Aurora, CO).

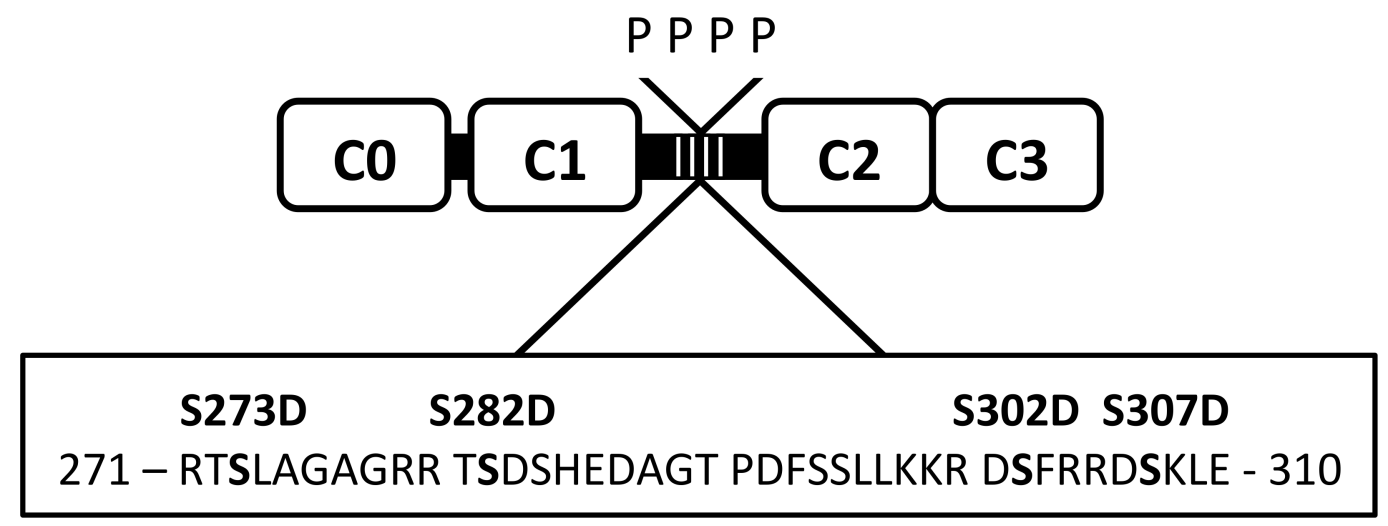

Figure 17: Diagram of $\mathrm{N}$-terminal cMyBP-C domains $\mathrm{C} 0$ through $\mathrm{C} 3$ that comprise the bacteriallyexpressed COC3 fragments. Fragment COC3 was expressed with the native serine residues in the $M$ motif. Four serines within the M-motif (S273, S282, S302, S207) were replaced with aspartic acids (D) to mimic phosphorylation in phosphomimetic fragment C0C3-4D. Biotinylated fragments were expressed containing a peptide tag-attached biotin sequence at the $\mathrm{N}$-terminus of domain $\mathrm{CO}$.

Similar to preparation of actin-coated microspheres, biotin-tagged C0C3 and C0C3-4D were coupled to streptavidin-coated microspheres by suspending a solution of microspheres in a saturating concentration of C0C3 or C0C3-4D, blocked with $1 \mathrm{mg} / \mathrm{ml}$ BSA to reduce nonspecific interactions, and were stored in actin buffer with $10 \mathrm{mM}$ DTT on ice at $4^{\circ} \mathrm{C}$ for up to 3 days. Microspheres were diluted immediately before use in actin buffer containing 10 mM DTT. An ultrasonic dismembrator was pulsed in diluted bead solution immediately before use to break apart clumps of microspheres. 


\section{Force spectroscopy}

The laser trap used in these experiments is described in Appendix 1, Section A1.2.

Coverslips coated with 3-10 $\mu \mathrm{m}$ glass spheres (targets) and then coated with nitrocellulose were prepared as in Guo and Guilford (2006) and assembled into flow cells.

For actin-COC3/4D bond lifetime measurements: $1 \mathrm{nM} \mathrm{C0C3}$ or C0C3-4D was incubated in the flow cell for 2 minutes. The flow cell was then blocked with $1 \mathrm{mg} / \mathrm{ml} \mathrm{BSA}$ in actin buffer for 2 minutes and washed with actin buffer. A suspension of actin buffer and biotinylated actin microspheres was added to the flow cell. Bond lifetime measurements were made between C0C3 or C0C3-4D on the flow cell surface targets and an actin-coated microsphere captured in the laser trap (Figure 18a).

For HMM-COC3/4D bond lifetime measurements: $10 \mu \mathrm{g} / \mathrm{ml} \mathrm{HMM} \mathrm{was} \mathrm{incubated} \mathrm{in} \mathrm{the} \mathrm{flow}$ cell for 2 minutes. The flow cell was then blocked with $1 \mathrm{mg} / \mathrm{ml} \mathrm{BSA}$ in actin buffer for 10 minutes and washed with actin buffer. A suspension of actin buffer and C0C3- or C0C3-4Dcoated microspheres was added to the flow cell. All HMM experiments were performed in the absence of nucleotide (in rigor). Bond lifetime measurements were made between HMM on the flow cell surface targets and a COC3- or C0C3-4D-coated microsphere captured in the laser trap (Figure 18b). 


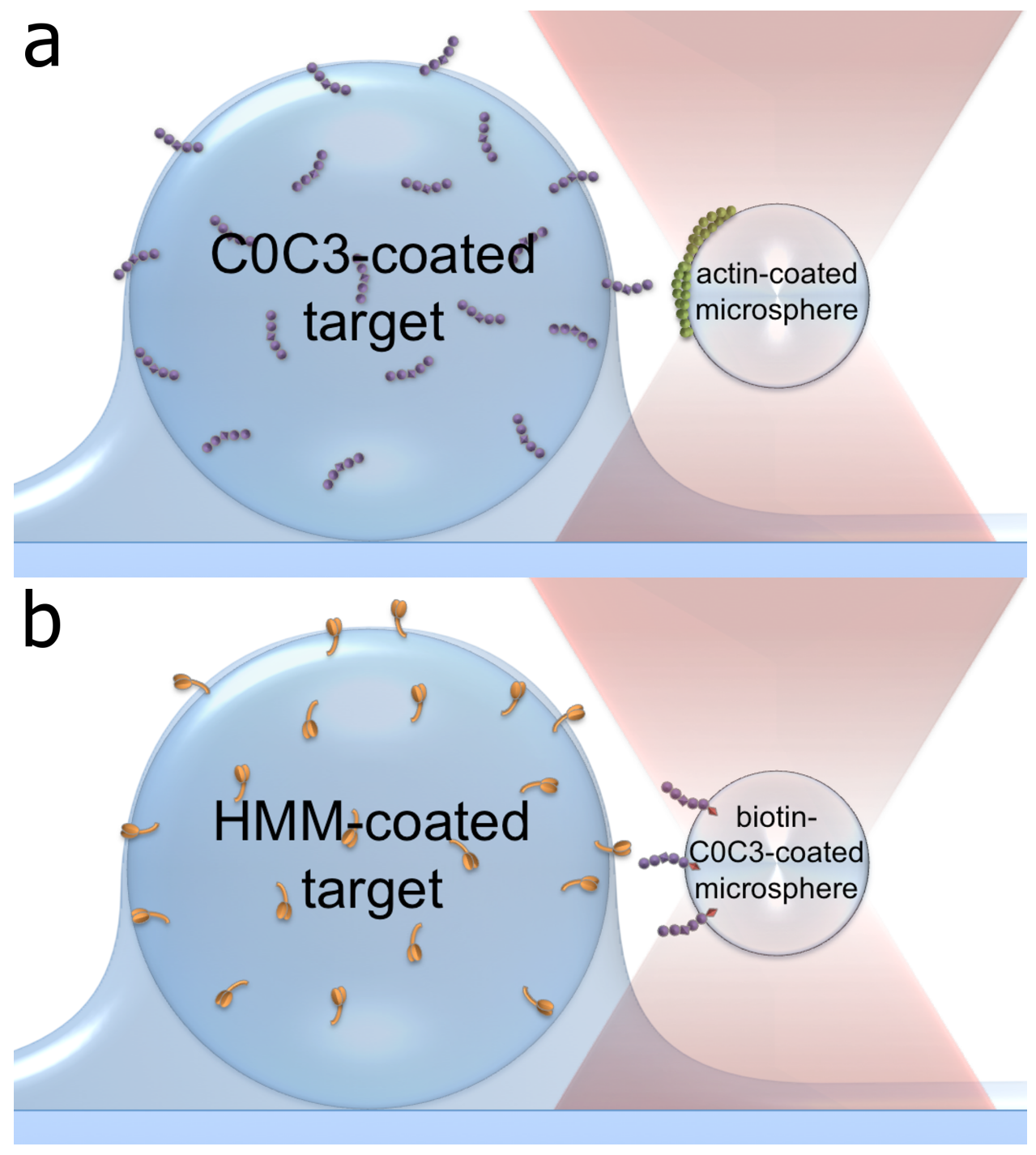

Figure 18: Experimental set up. a: A microsphere coated in biotinylated actin is captured in the laser trap. The nitrocellulose-covered target surface is coated in COC3 or COC3-4D. b: A microsphere is coated in biotinylated COC3 or COC3-4D. The nitrocellulose-covered target surface is coated in HMM.

Protein-coated microspheres were captured in a laser trap and brought into contact with a protein-coated target for 1 second (Figure 4). The laser trap was stepped $200 \mathrm{~nm}$ away from the target and held there for 1 second before repeating this cycle. The initial separation between the trapped microsphere and the target was random, resulting in random tensile loads experienced by the bond between actin or HMM and a cMyBP-C fragment. Over a large number of experiments this resulted in a range of random step loads being applied to actin or HMM-C0C3/4D bonds using the laser trap. Bond formation was 
accompanied by a decrease in Brownian motion and a small shift in displacement, as well as the trapped microsphere remaining in contact with the target after the laser trap was stepped away from it. Bond rupture was accompanied by return of the microsphere to its original position away from the target. Bond lifetime was defined as the time elapsed between stepping the laser away from the HMM-coated target and bond rupture. The tensile load experienced by a bond was calculated from the stiffness of the laser trap and the distance a microsphere moved to return to the center of the laser trap.

Control experiments were performed with blank targets (no C0C3- or C0C3-4D on targets with actin-coated microspheres, and no HMM on targets with C0C3- or C0C3-4D-coated microspheres).

\section{Zero load spectroscopy}

Bond lifetimes between actin or HMM and C0C3 or C0C3-4D at near-zero load were measured as described in Chapter 2, Section 2.

\section{Actin bond formation measurements}

The frequency of bond formation was measured over a range of COC3 surface densities to determine a concentration at which $50 \%$ or greater binding occurs. To measure the frequency of bond formation, an actin-coated microsphere was stepped in and out of contact with a C0C3-coated target surface with a range surface densities (50-400 nM C0C3). The frequency of bond formation was calculated as the number of trapped-microsphere target contacts resulting in a bond divided by the total number of contacts. Based on the results from these measurements, all bond formation experiments were performed while incubating $400 \mathrm{nM} \mathrm{COC3}$ or C0C3-4D in the flow cell.

The probability of bond formation was measured as a function of contact time between the actin-coated microsphere and the cMyBP-C fragment-coated target. Flow cells were assembled as previously described with C0C3 or C0C3-4D adsorbed to the nitrocellulosecoated target surface and biotinylated actin coupled to the laser-trapped microsphere. Similar to Chesla et al. (1998), we determined adhesion probability as a function of contact 
time between two surfaces by repeatedly stepping an actin-coated microsphere into contact with a stationary cMyBP-C fragment-coated target and counting the number of bonds formed for a fixed number of contacts. The molecules were stepped in and out of contact with a constant step-out time of 1000 ms and a range of contact (step-in) times (5$3200 \mathrm{~ms}$ ). The adhesion probability, the number of times a bond was observed divided by the total number of contacts, was plotted as a function of contact time. Adhesion probability $\left(P_{a}\right)$ data were fit according to Chesla et al. (1998) with the equation:

$$
P_{a}=1-\exp \left\{-A_{c} m_{r} m_{l} \frac{k_{f}^{0}}{k_{r}^{0}}\left[1-\exp \left(-k_{r}^{0} t\right)\right]\right\}
$$

where $A_{c}$ is the contact area, $m_{r}$ is the receptor density, $m_{l}$ is the ligand density, $k_{f}{ }^{0}$ is the specific rate of bond formation, $k_{r}{ }^{0}$ is the specific rate of bond rupture, and $t$ is the contact duration. Because $m_{r}$ and $m_{l}$ are as yet unknown to us, we fit our data by lumping unknown constants $A_{c}, m_{r}$, and $m_{l}$ with $k_{f}^{0}$ into one fitted parameter.

Time to bond formation $\left(t_{b}\right)$ was also measured to validate our adhesion probability measurements. $t_{b}$ was measured between single C0C3 or C0C3-4D binding sites on actincoated microspheres using the method described in Appendix 1.

cMyBP-C N-terminal fragment in vitro motility assay Actomyosin motility in the presence of the C0C3 cMyBP-C N-terminal fragments was measured as described by others (Saber et al., 2008; Weith et al., 2012a) at $30^{\circ} \mathrm{C}$. Briefly, 32 $\mu \mathrm{g} / \mathrm{ml} \mathrm{HMM}$ was applied to a flow cell made of a glass slide and nitrocellulose-coated coverslip separated by mylar shims. After a 1 minute incubation, the flow cell was blocked with $1 \%$ BSA in actin buffer with $10 \mathrm{mM}$ DTT for 1 minute, followed by addition of TRITCphalloidin labeled actin filaments. After a 1 minute incubation, the flow cell was washed with actin buffer with $10 \mathrm{mM}$ DTT and exchanged with a final motility buffer (25 mM KCL, 25 mM Imidazole, 1 mM EGTA, 4 mM MgCl 2,10 mM DTT, 1 mM ATP, 0.5\% Methyl Cellulose, $\mathrm{pH}$ 7.4) containing an oxygen scavenger system and a range of COC3 concentrations. The flow cell was incubated for 1 minute at $30^{\circ} \mathrm{C}$ before imaging. The mean velocities of 
filaments and the fraction of filaments that move were determined using the tracking algorithm described in Snook, et al. (2008).

\subsection{Results}

Phosphorylation does not modulate load-dependent cMyBP-C fragment bond lifetimes with actin

To determine if phosphorylation within the motif region can modulate the cMyBP-C Nterminal fragment's ability to inhibit actomyosin motility, we directly characterized cMyBP-C-actin interactions at the molecular level. Individual binding events between actin and either C0C3 or C0C3-4D were observed and the duration of these bonds (lifetime) were measured under an applied tensile load. Figure 19 shows that actin bond lifetime with both C0C3 and C0C3-4D decreases similarly over the range of tensile loads we tested. The bonds between actin and the $\mathrm{N}$-terminal fragments were each best fit with a single dissociation pathway from a single bound state (Bell, 1978). This increased rate of bond rupture with growing applied tensile loads, also known as slip bond behavior, has been widely observed in a variety of receptor-ligand pairs (Alon et al., 1995; Marshall et al., 2003; Merkel et al., 1999; Pierres et al., 1996).The fitted values for the unloaded bond dissociation rate for actin with COC3 and C0C3-4D were compared using a z statistic and were not statistically different $(p=0.4)$. 


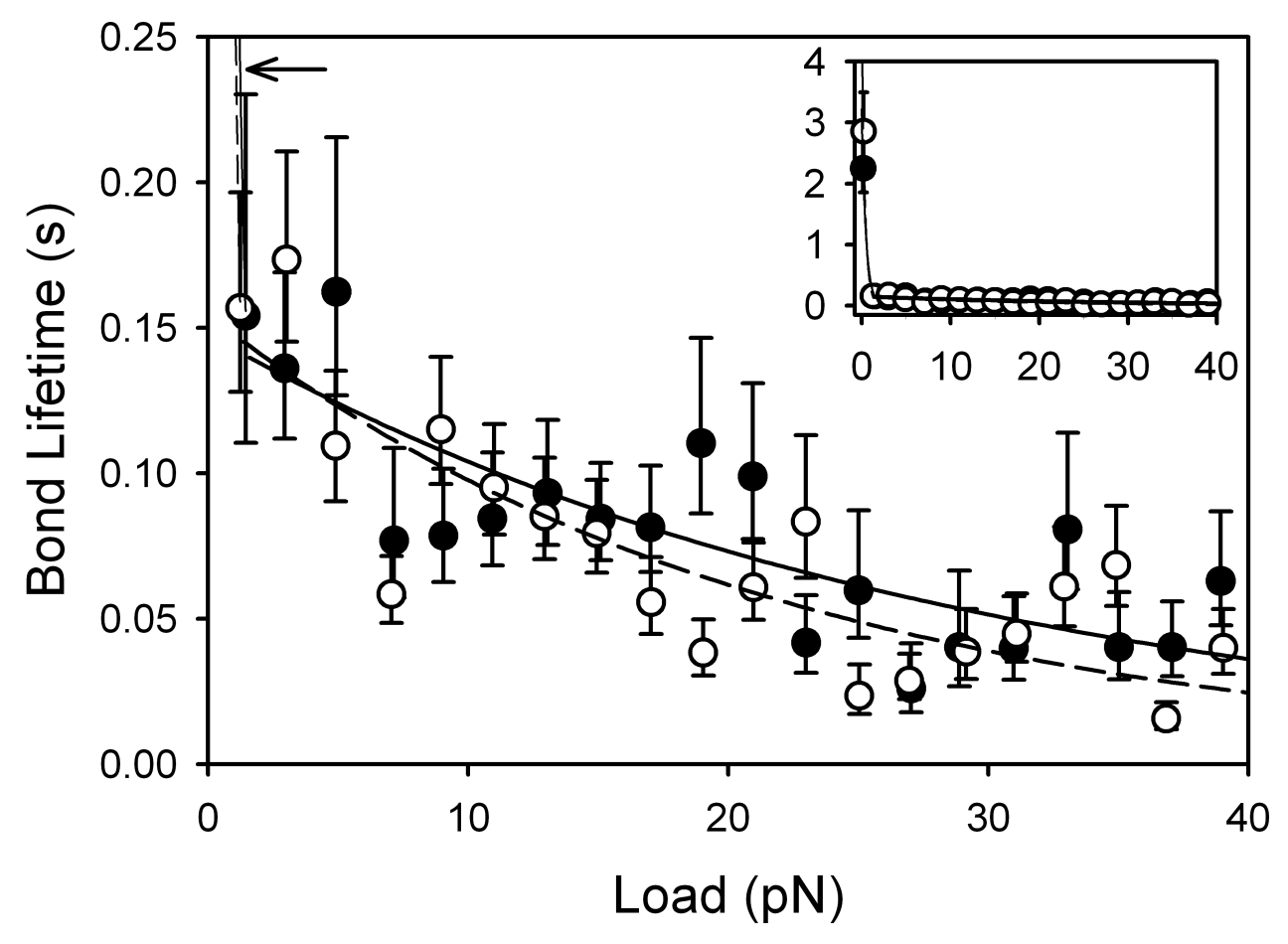

Figure 19: The duration of actin bonds with COC3 (solid circles) and COC3-4D (open circles) decrease similarly with applied tensile load. COC3 and COC3-4D data were fit by a single-pathway model (solid and dashed lines) (Bell, 1978). Near zero load data were fit by an exponential decay (unbold solid and dashed line, arrow) (Guo and Guilford, 2006). Near zero load data points are off the scale of the axis. Inset: Same as shown in full figure with vertical axis extended to include near zero load data. The mean number of bonds measured per $2 \mathrm{pN}$ force bin was 64 . Horizontal error bars represent the standard error of the mean load for each $2 \mathrm{pN}$ bin and most fall within the symbols. Vertical error bars represent the $95 \%$ confidence intervals of the exponentially distributed data.

As a control, the same measurement was performed between actin-coated microspheres and a blank target surface (no COC3 or C0C3-4D present). The control condition resulted in a flat bond lifetime profile over the range of loads measured, confirming that non-specific bonds are roughly load-independent (Guo and Guilford, 2006; Kong et al., 2009). The binding frequency - the fraction of bonds formed per those attempted - was lower for the control than for experimental conditions. The binding frequency was $2 \%$ for actin with blank targets, compared to 16 and 12\% for C0C3- and C0C3-4D-coated targets, respectively. These increased bond frequencies and response to load suggest that our bond lifetime measurements were specific between actin and C0C3 or C0C3-4D. 
To determine the bond lifetimes in the same physical system but at near-zero load, an actin-coated microsphere was captured and placed adjacent to the fragment-coated surface. Bonds were allowed to form and break with low, semi-random loads. The mean bond lifetimes at loads of only $0.18 \pm 0.02 \mathrm{pN}$ for C0C3 and C0C3-4D were $2.2 \pm 0.5$ and $2.9 \pm 0.9 \mathrm{~s}$, respectively ( $p=0.99$ ) (Figure 19, inset). Our zero load data are consistent with Weith et al. whose three-bead laser trap assay measured bond lifetimes between actin and C0C3 were indistinguishable from PKA-tread C0C3 phosphorylated at the same four serines that are modified in the current C0C3-4D fragment (Weith et al., 2012a).

The frequency of bond formation at zero load $\left(B_{0}\right)$ was calculated using the equation:

$$
B_{0}=n /\left(t_{\text {tootl }}-t_{\text {bound }}\right)
$$

where $n$ is the total number of bonds formed, $t_{\text {total }}$ is the duration of time for which the microsphere was adjacent to the surface (proteins were within binding range), and $t_{\text {bound }}$ is the duration for which binding was observed. $B_{0}$ was 0.006 bonds/s both for actin binding to COC3 as well as actin and C0C3-4D.

\section{Phosphorylation does not modulate load-dependent cMyBP-C bond lifetimes with HMM}

The inhibitory effect of N-terminal cMyBP-C on actomyosin motility and shortening velocities in fibers could also be explained by phospho-regulated $\mathrm{N}$-terminal binding to myosin by restricting the motion of the myosin head or by altering the kinetics of myosin's motion generation (Calaghan et al., 2000; Hofmann et al., 1991a). Therefore, we also directly characterized individual binding events between HMM and either C0C3 or C0C34D under an applied tensile load. Similar to the binding events with actin, bond lifetimes between HMM and C0C3 and those with C0C3-4D decreased equivalently over a range of tensile loads (Figure 20). The bonds between HMM and the fragments were best fit with a single dissociation pathway from a single bound state (Bell, 1978). The fitted values for the unloaded bond dissociation rate for HMM with C0C3 and C0C3-4D were compared using a $\mathrm{z}$ statistic and were not statistically different $(p=0.4)$. This is the first direct measurement of single molecule interactions between the N-terminal head region of myosin and cMyBP-C. 


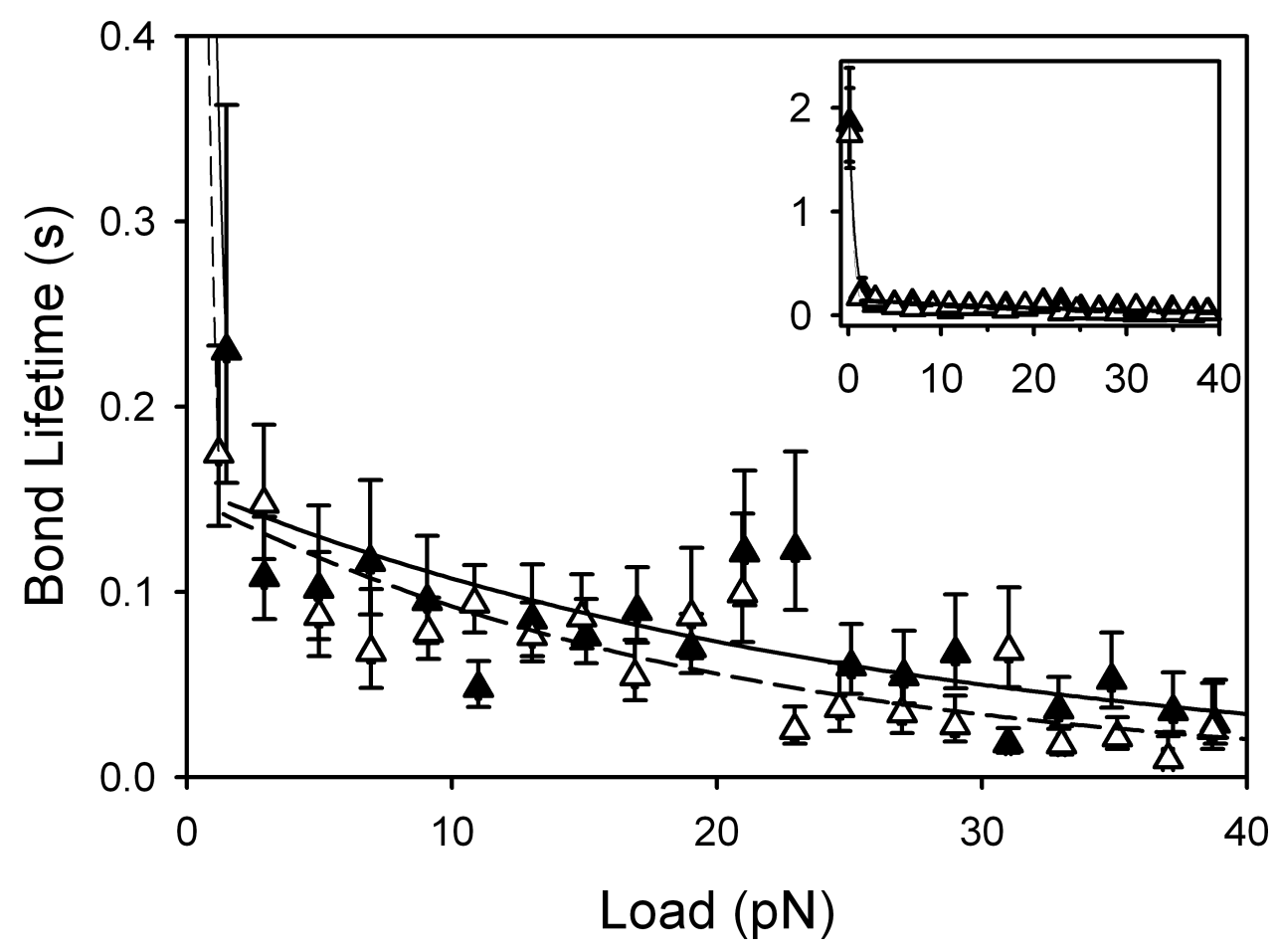

Figure 20: The duration of HMM bonds with COC3 (solid triangles) and C0C3-4D (open triangles) decreases with applied tensile load. COC3 and C0C3-4D data were fit by a single-pathway model (solid and dashed lines) (Bell, 1978). Near zero load data were fit by an exponential decay (unbold solid and dashed lines) (Guo and Guilford, 2006). Near zero load data points are off the scale of the axis. Inset: Same as shown in full figure with vertical axis extended to include near zero load data. The mean number of bonds measured per $2 \mathrm{pN}$ force bin was 44 . Horizontal error bars represent the standard error of the mean load for each $2 \mathrm{pN}$ bin and most fall within the symbols. Vertical error bars represent the $95 \%$ confidence intervals of the exponentially distributed data.

As a control, the same measurement was performed between C0C3-coated microspheres and a blank target surface (no HMM). The control condition resulted in a flat bond lifetime profile over the range of loads measured. The binding frequency was $4.5 \%$ for C0C3 with blank targets, compared to 16 and 13\% for HMM-coated targets with C0C3- and C0C3-4Dcoated microspheres, respectively.

Bond lifetimes between HMM and C0C3 or C0C3-4D were also measured at near-zero load as described for actin binding with the fragments. The mean bond lifetimes were $1.9 \pm 0.4$ and $1.7 \pm 0.3 \mathrm{~s}$ for C0C3 and C0C3-4D, respectively ( $p=0.5)$, under a mean load of 0.14 
$\pm 0.01 \mathrm{pN}$ (Figure 20, inset). $B_{0}$ did not differ between HMM binding with C0C3 or C0C3-4D, with a value of 0.01 bonds/s for each fragment.

Frequency of bond formation as a function of COC3 surface density

Bond formation in the laser trap must be measured at a higher surface density of the target protein (COC3 or C0C3-4D in these experiments) in order to overcome the limitations of diffusion in the ligand-receptor interaction, whereas bond lifetime measurements necessitate low site densities to ensure single bond events (Snook and Guilford, 2012). Therefore, the frequency of bond formation was measured over a range of C0C3 surface densities to find a concentration at which $50 \%$ or greater binding occurs.

A laser-trapped actin-coated microsphere was stepped in and out of contact with a COC3coated target surface. The number of bonds formed per total number of contacts was calculated to determine the frequency of bond formation for a range of C0C3 concentrations (50-400 nM) (Figure 21). Contact and step-out times were $1000 \mathrm{~ms}$ each for all experiments. The large error seen at $50 \mathrm{nM} \mathrm{C0C3}$ is due to a small sample size. Based on these data, all bond formation experiments were performed by applying $400 \mathrm{nM} \mathrm{C0C} 3$ or C0C3-4D to the surface of the flow cell. 


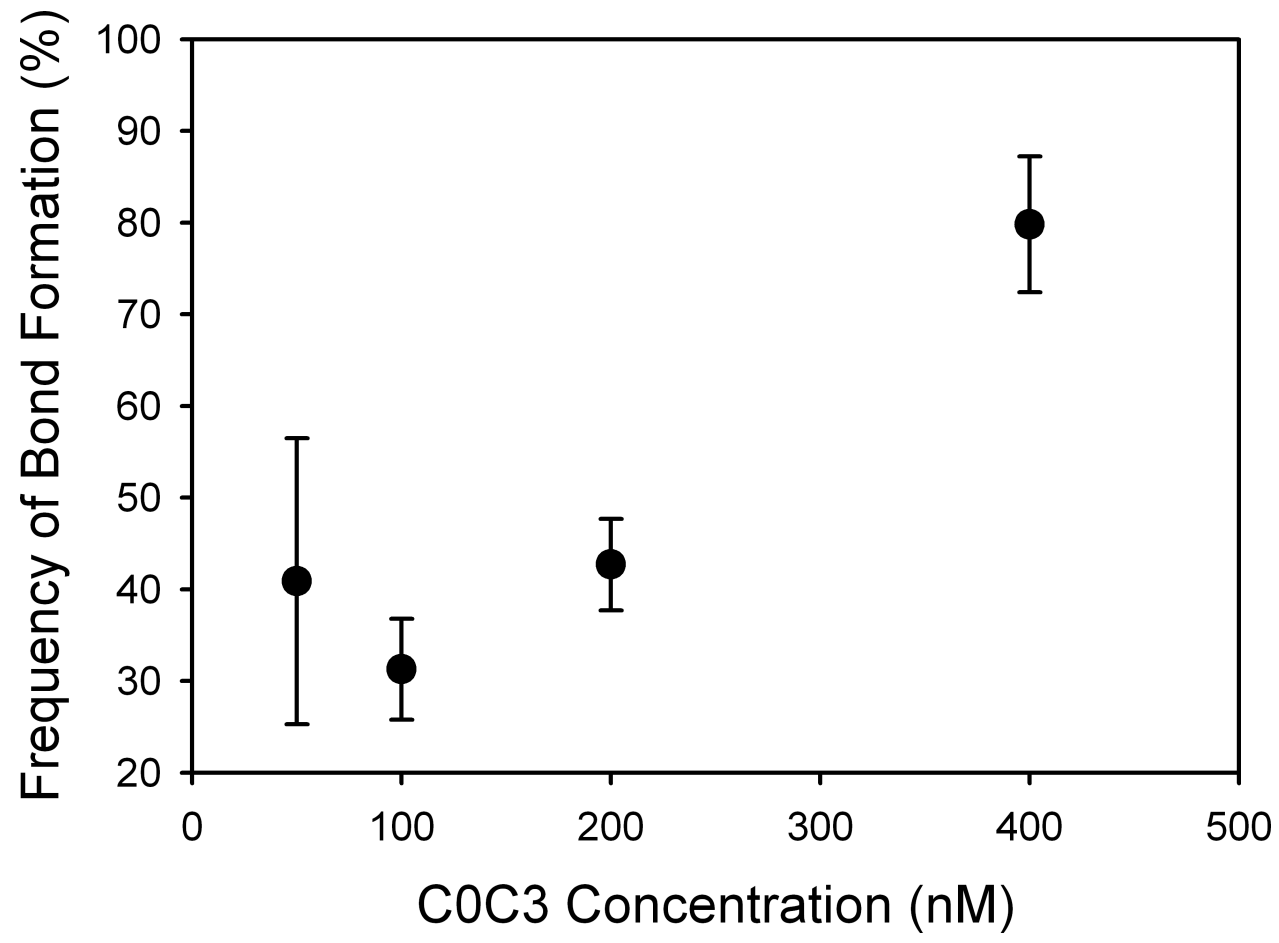

Figure 21: The frequency of bond formation was measured for actin with a range of C0C3 concentrations. Data are mean \pm standard error. The mean number of steps per data point is 594 .

Actin binding is unaffected by COC3 phosphorylation

To determine whether cMyBP-C phosphorylation affects actin-cMyBP-C bond formation rates, we measured the probability of bond formation as a function of fixed contact times between the actin-coated microsphere and C0C3 or C0C3-4D-coated target surface. Similar to Chesla et al. (1998), we counted the number of bonds formed for a fixed number of contacts over a range of contact times (5-3200 ms). Adhesion probabilities were plotted as a function of contact time (Figure 22). The resulting data were fitted as in Chesla et al. (1998) using Equation 7. 


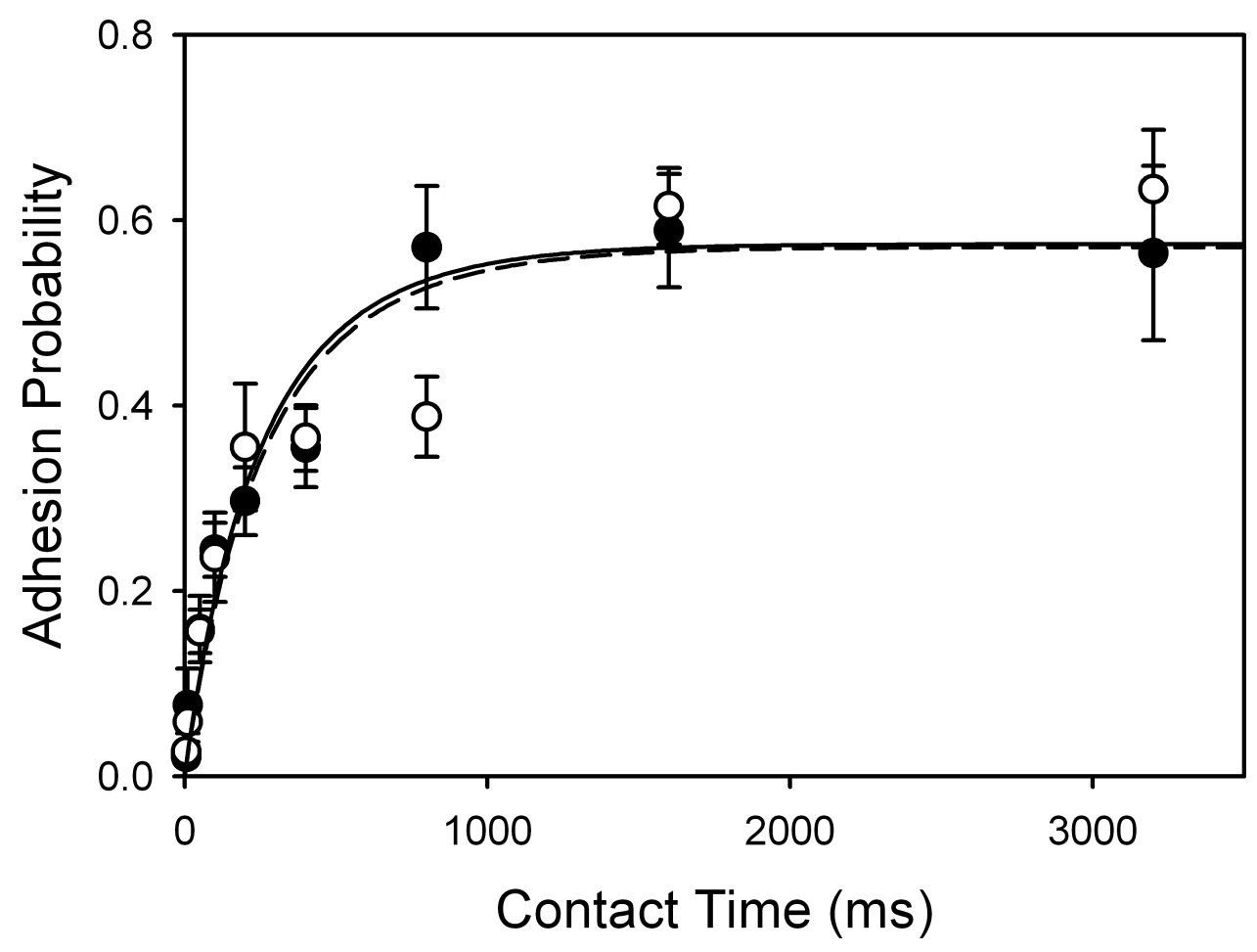

Figure 22: The adhesion probability plotted as a function of contact time for actin-coated microspheres making contact with C0C3- (black circles) or C0C3-4D- (open circles) coated targets. Adhesion probability was defined as the number of bonds formed divided by the total number of attempts. Equation 7 was fit to the Coc3 (solid line) and C0C3-4D (dashed line) data to determine the rate of bond formation. Data are mean \pm standard error. The mean number of steps per data point was 817.

Data were fit by lumping constants $A_{c}, m_{r}$, and $m_{l}$ with $k_{f}^{0}$ into one fitted rate parameter. The value of the fitted parameter was $2.4 \pm 0.7 \times 10^{-3} \mathrm{~ms}^{-1}$ and $2.3 \pm 0.6 \times 10^{-3} \mathrm{~ms}^{-1}$ for C0C3 and C0C3-4D, respectively. In addition to the specific 2D rate of bond formation, this parameter includes the contact area and receptor and ligand densities that are the same in both the C0C3 and C0C3-4D experimental conditions. Thus, any difference observed in this fitted parameter would result from a difference in bond formation rate.

Formation of a bond between actin on the trapped microsphere and C0C3 or C0C3-4D on the target surface can be directly observed. We therefore validated our relative rates of bond formation using an alternate technique in which we directly measured the time to bond formation $\left(t_{b}\right)$ between single C0C3 or C0C3-4D binding sites on actin-coated 
microsphere. $t_{b}$ was defined as the time elapsed between microsphere-target contact and the observed formation of a bond. Figure 23 shows the $t_{b}$ for actin binding with COC 3 and C0C3-4D, measured for observed bond formations in data traces collected from one flow cell at the $3200 \mathrm{~ms}$ contact time in the adhesion probability experiments described above. The mean $t_{b}$ was 0.59 and $0.68 \mathrm{~s}$ for actin binding with C0C3 and C0C3-4D, respectively ( $p=$ $0.9)$. The similar $t_{b}$ for the fragments binding with actin supports our adhesion probability finding of no difference in the rate of bond formation with actin between the unphosphorylated and phosphomimetic N-terminal cMyBP-C fragment.

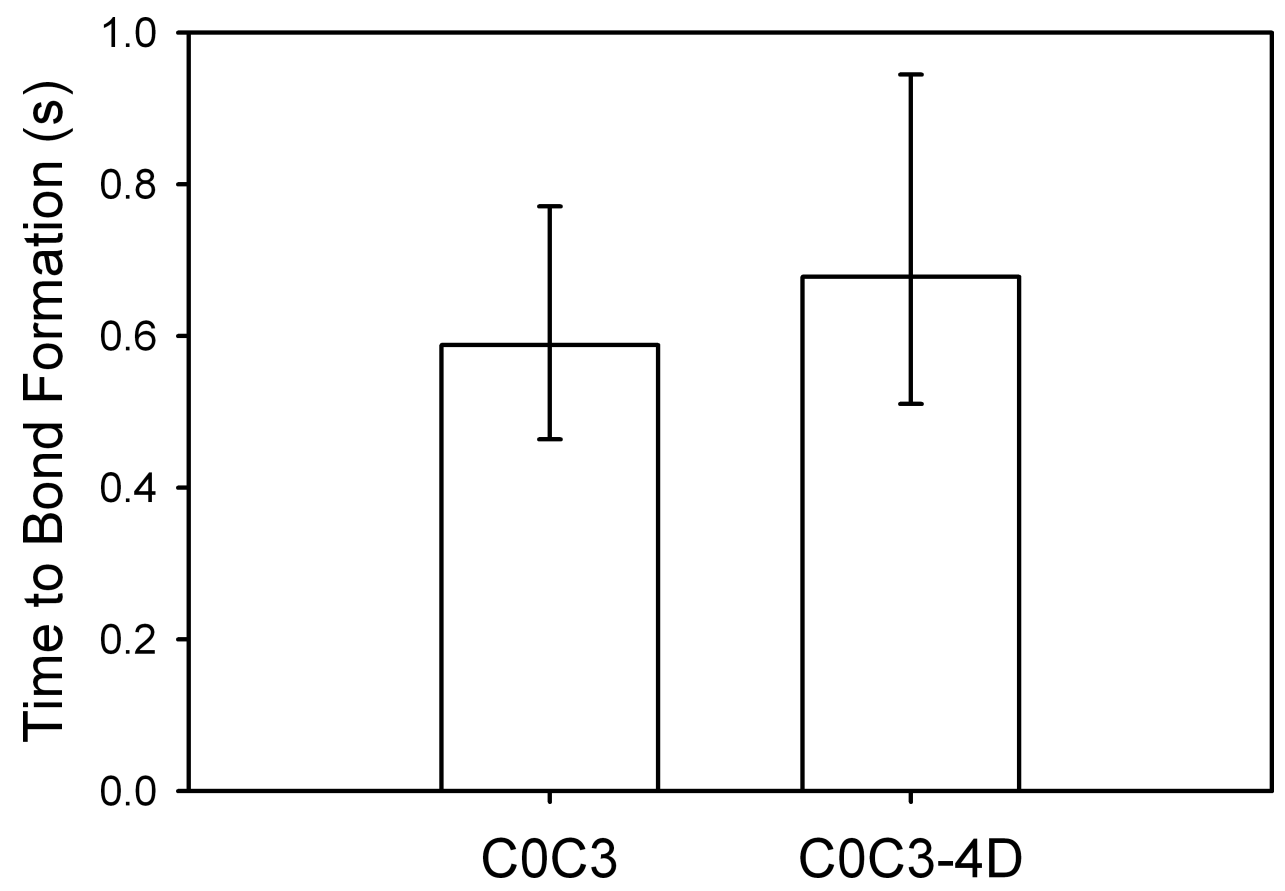

Figure 23: Time to bond formation measured at a representative contact time (3200 ms) was similar for actin with $\operatorname{Coc} 3(n=60)$ and $\operatorname{Coc} 3-4 D(n=41), p=0.9$. Error bars represent the $95 \%$ confidence intervals of the exponentially distributed data.

COC3 in vitro motility

Depressed motility has been observed by others with this (Weith et al., 2012a) and other C1 and M-motif-containing N-terminal cMyBP-C fragments (Razumova et al., 2006; Weith et al., 2012a). In order to confirm the activity of the fragment, we performed in vitro motility in the presence of C0C3 fragments over a range of concentrations $(0-1 \mu \mathrm{M})$. The 
HMM-propelled actin sliding velocity decreased as a function of the C0C3 concentration (Figure 24), while the fraction of filaments moving was similar regardless of C0C3 concentration (65 $\pm 2 \%$ ). Data were fit using

$$
v=\frac{v_{\max }}{1+\frac{[\mathrm{I}]^{n}}{k_{i}^{n}}}
$$

where $v$ is the velocity, $v_{\max }$ is the maximum velocity, [I] is the concentration of inhibitor, $k_{i}$ is the inhibitor concentration at $1 / 2 v_{\max }$, and $n$ is the Hill coefficient. $v_{\max }$ was experimentally determined to be $6.04 \mu \mathrm{m} / \mathrm{s}$ in the absence of C0C3. The fitted value of $k_{i}=0.81 \mu \mathrm{M}$ and the fitted value of $n=0.7$. 


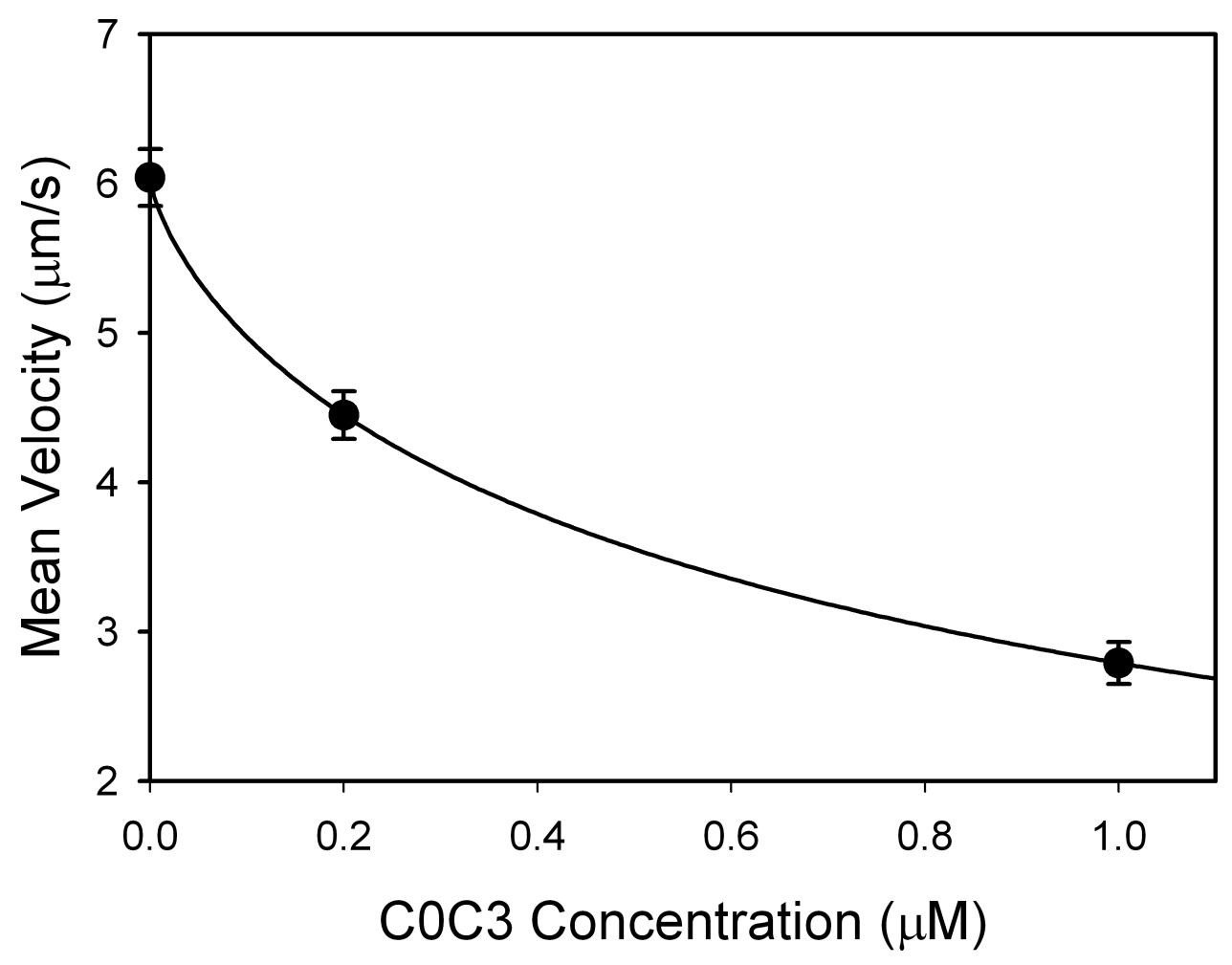

Figure 24: Concentration-dependent decrease in HMM-driven actin filament sliding velocity in the presence of C0C3 fragments (black circles). Data were fit using Equation 9. The fitted value of $k_{i}=0.81$ $\mu M$ and the fitted value of $n=0.7$. Data are mean $\pm \operatorname{standard}$ error $(n=9)$.

In vitro motility was not performed in the presence of the phosphomimetic C0C3-4D fragment due to the limited availability of protein fragments.

\subsection{Discussion}

Phosphorylation relieves inhibition of actomyosin motility caused by cMyBP-C even in the absence of thin filament regulatory proteins, revealing that the primary regulatory mechanism cMyBP-C must lie with one or both of the minimally essential crossbridge proteins - actin and myosin. There are two main theories of where this regulatory interaction occurs. One theory suggests that $\mathrm{N}$-terminal cMyBP-C reduces actin filament sliding by imposing a direct drag on the actin filament. The second suggests that $\mathrm{N}$-terminal cMyBP-C alters the kinetic rates or range of motion of the myosin head. Whatever its binding partner may be, and whatever its mechanism of action may be, phosphorylation reverses the functional effects of cMyBP-C. Therefore, a difference in the interaction 
between phosphorylated or unphosphorylated N-terminal cMyBP-C with actin or myosin should point to the physiological crossbridge binding partner of cMyBP-C. Surprisingly we saw no such difference with either actin or myosin as the binding partner.

Our data do not support the notion that cMyBP-C acts as a phosphorylation-dependent drag on the actin filament. Neither the rate of actin binding with COC3 nor the rate of unbinding is affected by aspartic acid replacement of the four phosphorylatable M-motif serines. If cMyBP-C did exert a phosphorylation-dependent drag, then we would have expected to see significantly longer bond lifetimes for the C0C3 fragment than C0C3-4D. We are confident that the lack of observed difference in the C0C3 and C0C3-4D fragments does not stem from the use of phosphomimetic fragments instead of PKA-phosphorylated C0C3. The quadruple-site phosphomimetic has been shown to impart a similar effect on actomyosin function as PKA-treated C0C3 (Weith et al., 2012b), which also agrees with the effect of phosphomimetic cMyBP-C in fiber studies (Sadayappan et al., 2009). It is therefore unlikely that the actin binding and unbinding rates with the use of C0C3-4D would differ from those of PKA-treated C0C3.

The only measurements showing that phosphoregulation of N-terminal cMyBP-C directly influences its binding with actin are performed in truly unloaded conditions (Shaffer et al., 2009; Weith et al., 2012a). However, there is contradictory evidence that cMyBP-C-actin binding is independent of the cMyBP-C phosphorylation state (Rybakova et al., 2011). Moreover, protein interactions often behave differently in the absence of an externally applied load. In the absence of load, thermal energy experienced by the molecules in solution determines the rate of bond formation and rupture.

This is one of the first direct measurements of these kinetic rates under loaded conditions between actin and N-terminal cMyBP-C. Weith et al. measured single-molecule bond lifetimes between actin and cMyBP-C fragments but did not measure them as a function of applied load (Weith et al., 2012a). Our data suggest that small changes in applied load have a marked effect on the actin-C0C3 bond lifetime, particularly as applied loads approach zero load. Therefore, it is difficult to directly compare the bond lifetimes measured in the 
current study to those observed by Weith et al. However, both studies agree that C0C3 phosphorylation or phosphomimetic replacement do not alter the actin-C0C3 bond lifetime compared to unphosphorylated C0C3.

This begs the question, if phosphorylation-mediated drag on the actin filament is not causing the functional regulation of crossbridge kinetics, what is?

We repeated our single molecule force spectroscopy measurements to determine if this interaction with myosin recommends it as the phosphorylation-dependent crossbridge binding partner of cMyBP-C. This is the first direct, load dependent measurement of the bond between N-terminal cMyBP-C and HMM (which contains S2 as well as the actinbinding S1 domains of myosin). Our results at near zero load and over a range of applied tensile loads suggest that the $\mathrm{N}$-terminus of $\mathrm{cMyBP}-\mathrm{C}$ can bind to HMM and that it does so in a load-dependent manner, but like its bond with actin the COC3-HMM bond is unresponsive to phosphomimetic replacement in the M-motif.

N-terminal cMyBP-C has been shown to bind myosin S2 through its M-motif (Gruen and Gautel, 1999) and C1 domain (Ababou et al., 2008), and lower affinity binding has been observed in the $\mathrm{C} 2$ domain as well (Ababou et al., 2007). There is evidence that cMyBP-C Mmotif phosphorylation modulates this binding by altering the charge or shape of the disordered region, resulting in a decreased affinity for S2 (Gruen et al., 1999). Like the binding assays described for actin, these measurements showing phosphorylationdependent binding between $\mathrm{N}$-terminal cMyBP-C and S2 were in an unloaded environment. This is not representative of the mechanical implications of cMyBP-C acting as a viscous load on the actomyosin crossbridge, or of the forces experienced by myosin bound to actin in the crossbridge as a result of the action of parallel crossbridges. Our load-dependent measurements of bond lifetime suggest that the $\mathrm{C} 0 \mathrm{C} 3$ region of $\mathrm{cMyBP}-\mathrm{C}$ contains a region that binds to HMM and that this bond is unchanged by phosphorylation. Given the physiological loads experienced by the proteins, we think this is a more likely representation of their true response (or lack thereof) to phosphorylation. 
The surface on which the COC3 and C0C3-4D fragments were present was reversed between the actin and HMM laser trap assays. HMM was adsorbed to the nitrocellulosecoated target surface because HMM function is disrupted when it is coupled to streptavidin-coated microspheres; though evidence of this is anecdotal, it comes from several major labs. cMyBP-C fragments were therefore biotinylated and coupled to streptavidin-coated microspheres. In contrast, actin was biotinylated and coupled to streptavidin-coated microspheres instead of being adsorbed to the nitrocellulose-coated target, and in this instance C0C3 or C0C3-4D was adsorbed to the nitrocellulose-coated target. There were several reasons for this reversal of surfaces. The site of biotinylation lies at the $\mathrm{N}$-terminal end of the $\mathrm{C} 0$ domain and when coupled to a streptavidin-coated microsphere would orient the fragment so that the $\mathrm{C} 0$ domain is closest to the bead surface with the remaining domains extending radially from the microsphere. It is questionable whether this biotinylated fragment could even be used for actin binding experiments since cMyBP-C-actin binding has been observed in domains C0 through C2 (Kulikovskaya et al., 2003; Lu et al., 2011; Razumova et al., 2006; Shaffer et al., 2009; Squire et al., 2003; Whitten et al., 2008), and biotinylation at the $\mathrm{C} 0$ domain would orient the actin-binding portion of the protein away from the actin filament. Furthermore, the presence of biotin on the $\mathrm{CO}$ domain may interfere with actin binding at this domain. Reversing the protein-coated surfaces relative to one another for actin compared to HMM binding assays partially alleviates these concerns. However, the concern that a difference in accessibility and behavior between adsorbed and biotin-coupled fragments remains.

Our bond lifetime data suggest that phosphorylation does not alter the actin-C0C3 off-rate, but this experiment is only able to make observations about bonds that have already formed. One might posit that the N-terminus of cMyBP-C fluctuates between two states - a binding (open conformation) and a non-binding (closed conformation) state - and that phosphorylation biases the molecule toward the closed conformation. Indeed, the mechanical stability of cMyBP-C is altered by phosphorylation in a way that suggests the natively freely extensible M-motif (Karsai et al., 2011, 2013) takes on a more stable conformation (Michalek et al., 2013). Force-extension relationships measured using atomic force spectroscopy show that phosphorylation reduces the mean contour length of the 
protein by $24 \mathrm{~nm}$ (Michalek et al., 2013). This shortening suggests that either the negative charge introduced by phosphorylation establishes an electrostatic interaction within the motif, and/or that the motif adopts new secondary structural elements.

Nevertheless, our relative rate of bond formation measurements for actin with C0C3 and C0C3-4D were indistinguishable, suggesting that the dynamic conformational changes that are stabilized by M-motif phosphorylation do not affect the availability of the actin binding site and therefore the actin-cMyBP-C rate of bond formation. Microscopic reversibility suggests that molecules with similar rates of bond rupture also exhibit similar rates of bond formation. It is therefore unsurprising that the relative bond formation rates of actin binding with $\mathrm{COC} 3$ and with C0C3-4D are indistinguishable since the rates of bond rupture were also indistinguishable. While there is growing evidence of direct interaction between $\mathrm{N}$-terminal cMyBP-C and the thin filament (Mun et al., 2014), we have no reason to suspect the myosin head alone as the direct binding partner for $\mathrm{CMyBP}-\mathrm{C}$-crossbridge regulation. Therefore, we did not measure the rate of bond formation between HMM and N-terminal cMyBP.

When compared to control data, the observed load-dependence of bond lifetimes and increased frequency of bond formation suggest that the bonds we measured are in fact between actin or HMM and COC3 or C0C3-4D, and not the result of non-specific interactions between a protein and the blocking agent or the opposing surface. Our data therefore are measuring specific protein-protein interactions.

Although our data seem to refute the two prominent hypotheses of cMyBP-C crossbridge regulation, we feel our data do not disagree with existing evidence given that our data were collected at finite loads, not zero load conditions.

The literatures present a body of confusing and seemingly conflicting pieces of evidence could be explained by an obvious crossbridge binding partner that has not yet been directly examined - the actomyosin complex. Crossbridge function may not be regulated by cMyBP$\mathrm{C}$ binding to actin or to myosin alone, but would require the actomyosin complex for 
specific binding and regulation of the crossbridge. If that were the case, it could explain why actin and myosin bind with varying affinities to the same regions of cMyBP-C and why phosphorylation-dependent changes in the bond are not observed with actin or myosin alone.

Mutations in many sarcomeric proteins lead to familial hypertrophic cardiomyopathy (FHC), including myosin, troponin, and cMyBP-C (Bonne et al., 1998). Mutations in the cMyBP-C gene MYBPC3 are the most common cause of FHC, accounting for approximately 200 mutations all linked to the MYBPC3 gene (Harris et al., 2011). A mutation at the actinmyosin binding interface, though not in loop 2, causes the most rapidly fatal form of FHC. Individuals with FHC exhibit myocyte enlargement, cellular disorganization, fibrosis, and reduced cardiac contraction and relaxation, though the mechanism of disease related to many of these mutations is unknown.

FHC mutations manifest in varied phosphorylation states of crossbridge-associated proteins, including cMyBP-C, suggesting that more than the protein itself, but also its prosttranslational modifications are critical to maintaining cardiac function (Barefield and Sadayappan, 2010). Interestingly, when constitutively phosphorylated full-length cMyBP-C is able to restore normal cardiac function in a cMyBP-C-null background, and its presence can even protect tissue from ischemia-reperfusion injury (Sadayappan et al., 2006). This data suggest that cMyBP-C phosphorylation may even be cardioprotective.

\subsection{Conclusion}

In Aim 2A, we hypothesized that cMyBP-C acts as a phosphorylation-reversible viscoelastic drag on the actin filament in vitro. Our direct measurement of indistinguishable bond lifetimes between unphosphorylated or phosphomimetic N-terminal cMyBP-C and actin refutes this hypothesis. Similar bond lifetime measurements between HMM and unphosphorylated or phosphomimetic N-terminal cMyBP-C were also indistinguishable, refuting our proposal that cMyBP-C imposes a phosphorylation-dependent regulation of myosin crossbridge activity. This suggests that $\mathrm{cMyBP}-\mathrm{C}$ crossbridge regulation may 
require the actomyosin complex as a binding partner in order to mechanically or kinetically alter crossbridge cycling. 


\section{Chapter 4: Exploring the functional effect cMyBP-C nitrosylation}




\subsection{Introduction}

Regulatory proteins are themselves invariably regulated. In the myofibrillar apparatus, many proteins rely on regulation by phosphorylation to tune functional interactions with other myofibrillar proteins in order to regulate muscle contraction, as described for cMyBP-C in the previous chapter. Nitrosylation, like phosphorylation, has been shown to regulate cardiac contractility. However, as discussed in Chapter 1, the individual protein targets of nitrosylation and their functional consequences are largely unknown.

cMyBP-C nitrosylation is thought to occur exclusively in the C-terminal domain of the protein (Figueiredo - Freitas et al., 2013) but its functional effect, if any, has not been tested. The C-terminal domain of cMyBP-C is the light meromyosin (LMM)-binding domain. cMyBP-C C-terminal interactions with the thick filament are necessary for normal myocyte structure and organization, though it is not required for cardiac development or sarcomere assembly (Harris et al., 2002; McConnell et al., 1999; Yang et al., 1998). Different variants of hetero- and homozygous mutations and deletions of cMyBP-C directed at the C-terminal domains show that loss of thick-filament binding capability results in disorganized sarcomeres and severely reduced cardiac function. These mutations are analogous to defects in humans that cause hypertrophic cardiomyopathy and result in similar structural and functional consequences.

cMyBP-C is thought to have two primary points of interaction in the cardiac sarcomere - its $\mathrm{C}$-terminus anchors it to the thick filament and its $\mathrm{N}$-terminus regulates the actomyosin crossbridge. We sought to isolate the effects of cMyBP-C nitrosylation on each of these functional interactions to understand the role of NO in regulating cMyBP-C and the contractile apparatus. 


\subsection{Methods}

\section{Proteins}

HMM, LMM, and actin were prepared as described in Appendix 1, Section A1.1. Actin filaments were stabilized with TRITC-labeled phalloidin. Bacterially expressed cMyBP-C and the C0C3 fragment were obtained from the Warshaw Lab (University of Vermont) and were prepared as described in Appendix 1, Section A1.1.

\section{Coumarin switch}

A coumarin switch procedure for in-gel visualization of nitrosylated proteins was performed to quantify the number of cysteines in cMyBP-C and C0C3 able to be nitrosylated in vitro as described in Appendix 1, Section A1.4. NO donors S-nitroso-Lcysteine (SNO-L-cys), L-nitrosoglutathione (GSNO), and diethylamine-NONOate (DEANONOate) were tested. SNO-L-cys and GSNO are endogenous donors of NO, while DEANONOate is a synthetic donor of free nitrosonium ion.

In vitro motility assay

cMyBP-C nitrosylation was performed using the donor SNO-L-cys at a final concentration of $400 \mu \mathrm{M}$ and allowed to react for 5 minutes in the dark. Treated and untreated cMyBP-C were diluted in motility buffer and placed on ice under vacuum for 20 minutes to remove nitric oxide. A cMyBP-C-free control sample was prepared in which a volume of donor equal to that used to treat cMyBP-C samples was added directly to a volume of motility buffer prior to degassing.

DTT-free actomyosin motility was measured as described by others (Snook et al., 2008) with modifications to include cMyBP-C (Saber et al., 2008). Briefly, rat skeletal HMM in 10 mM DTT was applied to a flow cell. After a 1 minute incubation the flow cell was blocked with $1 \mathrm{mg} / \mathrm{ml}$ BSA in actin buffer with $10 \mathrm{mM}$ DTT for 1 minute, followed by the addition of TRITC-phalloidin labeled rat skeletal actin filaments. After a 1 minute incubation, the flow cell was washed with DTT-free vacuum degassed actin buffer. One volume of DTT-free degassed motility buffer containing treated or control cMyBP-C was added. Actin filament 
movement was imaged at $30^{\circ} \mathrm{C}$ and mean velocities of filaments and the fraction of filaments that move were determined using the tracking algorithm described in Snook et al. (2008).

Motility was measured using control and donor-treated cMyBP-C. All reducing-agent-free buffers were degassed under vacuum for 20 minutes in small aliquots to remove oxygen. Solutions were re-degassed after 4 flow cells or 20 minutes, whichever came first.

\section{LMM filament assembly and cMyBP-C binding}

cMyBP-C nitrosylation was performed as above. Two control samples of filamentous LMM were also prepared in the absence of CMyBP-C - one in which LMM was allowed to react with donor and one in which decomposed donor was added. Nitrosylation was performed before cMyBP-C was combined with LMM filaments.

cMyBP-C binding to LMM was measured using an LMM centrifugation assay (Okagaki et al., 2007). Briefly, rat skeletal LMM was dialyzed against a low-salt buffer to allow filament formation. Upon removal from dialysis, LMM was combined with a sub-saturating amount of $\mathrm{cMyBP}-\mathrm{C}$ or nitrosylated $\mathrm{CMyBP}-\mathrm{C}$ and incubated on ice for 30 minutes to allow binding. This concentration of cMyBP-C was chosen based upon a saturable MyBP-C binding curve (Moos et al., 1975; Okagaki et al., 2007) so as not to introduce saturation artifacts. Samples were centrifuged at $100,000 \mathrm{~g}$ for 40 minutes at $4^{\circ} \mathrm{C}$, at which speed filamentous LMM and bound cMyBP-C pellet while unbound cMyBP-C and monomeric LMM remain in the supernatant. Pellet and supernatant were collected and resuspended in sample buffer. Prior to addition of sample buffer, pellets were resuspended in a volume of low-salt buffer equal to the sample volume prior to centrifugation to allow for quantitative comparison. Gel electrophoresis was performed on the pellet and supernatant from all samples.

Gels were stained with colloidal coomassie and the volume of each LMM and cMyBP-C band was measured for each sample condition. 


\subsection{Results}

Quantification of cMyBP-C nitrosylation

cMyBP-C is able to be nitrosylated in vitro. SNO-L-cys and GSNO nitrosylated cMyBP-C similarly at 4 of the 22 cys residues present in the cMyBP-C sequence (UniProt accession No. 070468; Table 1).

The N-terminal C0C3 fragment was unable to be nitrosylated by any of the three donors tested, though there are 7 cys residues present in this shortened sequence. These data suggest cMyBP-C nitrosylation in full-length cMyBP-C occurs exclusively in the C-terminal region of the protein. Our data are consistent with mass spectroscopy of nitrosylated cardiac myofibril proteins that has identified four nitrosylatable cysteine residues in the C10 domain of cMyBP-C (Figueiredo-Freitas et al., 2013). 
Table 1: Number of cysteine residues able to be nitrosylated in cMyBP-C and COC3 in vitro by endogenous NO donors

\begin{tabular}{lcc}
\hline & \multicolumn{2}{c}{ Number of cys Nitrosylated } \\
\cline { 2 - 3 } & cMyBP-C (22) & COC3 (7) \\
\hline SNO-L-cys & 3.6 & 0.4 \\
GSNO & 4.2 & 0.2 \\
DEA-NONOate & - & 0
\end{tabular}

(n) is the total number of cys residues present in the protein or fragment sequence acquired from UniProt, accession No. 070468. Number of cysteine residues nitrosylated is averaged from two gelloading volumes for one coumarin switch for CMyBP-C and two coumarin switches for COC3. DEANONOate was not tested as an NO donor for cMyBP-C. Values are calculated using Equation 19.

Nitrosylation does not affect the ability of cMyBP-C to regulate actomyosin motility In the in vitro motility assay, cMyBP-C slows the velocity of HMM-driven actin filament sliding in a concentration-dependent manner (Saber et al., 2008). We investigated whether nitrosylation of cMyBP-C alters its regulation of actomyosin motility. No difference in actin motility was observed between untreated and nitrosylated cMyBP-C over range of surface densities (Figure 25). The mean velocity of treated and untreated cMyBP-C samples both displayed a concentration-dependent inhibitory trend similar to that reported in Saber et al. (2008). These data suggest that cMyBP-C nitrosylation has no functional effect on the interaction of cMyBP-C with the actomyosin crossbridge. This is unsurprising given our evidence that $\mathrm{CMyBP}-\mathrm{C}$ nitrosylation is localized to the C-terminus, which interacts with the myosin tail (which was absent in these experiments) rather than the crossbridge. 


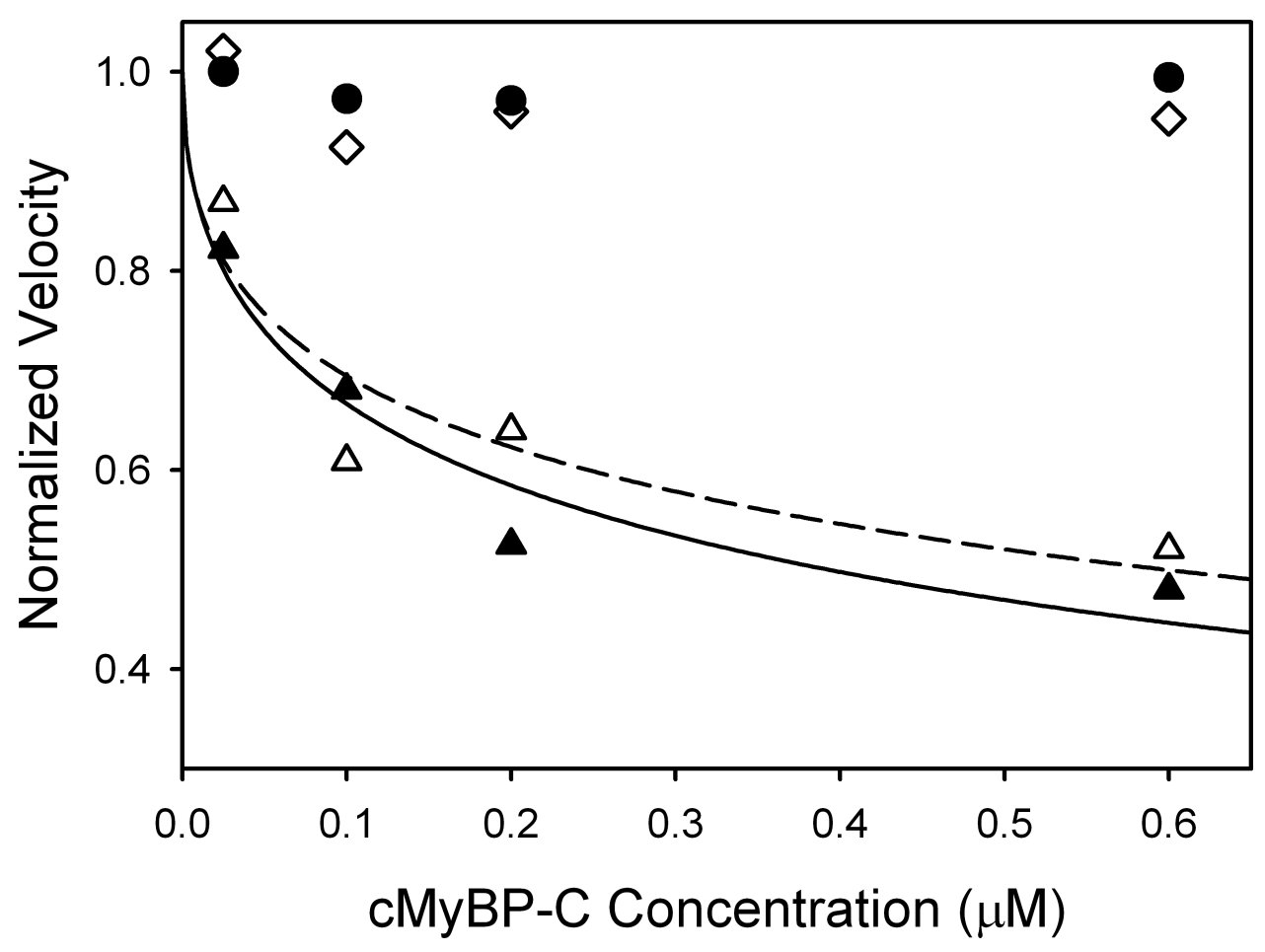

Figure 25: Changes in HMM-driven actin filament velocity as a function of cMyBP-C (solid triangles) or cMyBP-C treated with $400 \mu$ M SNO-L-cys (open triangles). Actin-HMM motility in the absence of cMyBP-C (solid circles) was measured with additions of actin buffer to match the added sample volumes for each concentration. Actin-HMM motility with volume-matched decomposed donor (open diamonds) was measured as a control. These data demonstrate that cMyBP-C functional regulation of the actomyosin crossbridge is unaffected by cMyBP-C nitrosylation. Data in the presence of cMyBP-C were fit using Equation 9 with $v_{\max }=1$ for the normalized value. The fitted values for $\boldsymbol{k}_{i}$ for $\mathrm{cMyBP}-\mathrm{C}$ and nitrosylated cMyBP-C were $0.5 \pm 0.1$ and $0.5 \pm 0.2 \mu \mathrm{M}$, respectively. The fitted values for $n$ were $0.4 \pm 0.1$ and $0.6 \pm 0.3$, respectively.

As expected, actomyosin motility was uninhibited in the absence of cMyBP-C. Control experiments in which decomposed donor was present in the motility buffer exhibited motility similar to that of actomyosin alone, suggesting that actomyosin motility is unaffected by decomposed donor remaining in the solution after treatment.

\section{LMM filament assembly and cMyBP-C binding are unaffected by nitrosylation}

cMyBP-C binding with LMM filaments was investigated by measuring the fraction of untreated and nitrosylated CMyBP-C that were bound to LMM using coprecipitation and centrifugation. After binding and centrifugation, sample supernatant and pellet band 
volumes were measured from brightfield gel images and used to calculate the pelleted fraction of protein for LMM and cMyBP-C in each sample using Equation 20 (Table 2). SNOL-cys treated and untreated cMyBP-C binding resulted in a similar pelleted fraction of cMyBP-C -0.19 and 0.15 , respectively - suggesting that nitrosylation does not affect the binding of cMyBP-C to the myosin tail.

Table 2: Pelleted fraction of assembled LMM filaments and bound cMyBP-C

LMM Pelleted Fraction cMyBP-C Pelleted Fraction

$\begin{array}{lcc}\text { LMM \& cMyBP-C } & 0.64 & 0.19 \\ \text { LMM \& cMyBP-C.N } & 0.42 & 0.15 \\ \text { cMyBP-C } & - & 0.04 \\ \text { LMM } & 0.89 & - \\ \text { LMM.N } & 0.92 & - \\ \text { LMM \& Decomposed Donor } & 0.98 & -\end{array}$

Pelleted fraction $\mathrm{P}=\mathrm{V}_{\text {pelete }} /\left(\mathrm{V}_{\text {pelete }}+\mathrm{V}_{\text {superatant }}\right) \cdot \mathrm{P}$ is the mean of two independently prepared centrifugation assays except for LMM \& Decomposed Donor and LMM.N samples, which are the value obtained in one centrifugation assay.

In samples containing both LMM and cMyBP-C, LMM pelleted fraction is reduced from 0.89 to 0.64 and 0.42 for untreated and treated cMyBP-C, respectively. An obvious interpretation is that the LMM filaments partially disassemble in the presence of cMyBP-C. A similar binding assay between LMM and the skeletal MyBP-C C10 domain suggests that the LMM polymerization equilibrium is sensitive to the ionic strength and ratio of LMM to cMyBP-C (Miyamoto et al., 1999). This could explain the decrease in assembled LMM filaments and therefore the reduced pellet fraction observed in the presence of cMyBP-C. 
As a control, the same measurement was performed for donor-treated LMM as well as LMM mixed with decomposed donor, both in the absence of cMyBP-C. As expected, both control conditions resulted in a pelleted fraction similar to that of LMM alone, where LMM was found primarily in the pellet. It is therefore unsurprising that direct SNO-L-cys treatment of LMM did not alter LMM filament assembly.

\subsection{Discussion}

Full length cMyBP-C was able to be directly nitrosylated in vitro at 4 of the 22 total cys residues by two small, naturally occurring nitrosothiols. In contrast, treatment of C0C3, the $\mathrm{N}$-terminal and purported crossbridge-binding fragment of cMyBP-C, using the same donors and free nitric oxide resulted in no significant S-nitrosylation of the 7 cys residues present. These data suggest that the 4 nitrosylatable residues reside in the $\mathrm{C}$-terminus of the protein. It was therefore unsurprising that the established inhibition of actomyosin motility observed in the presence of cMyBP-C was unaffected by cMyBP-C nitrosylation.

Mass spectroscopy of nitrosylated cardiac myofibril proteins localized 4 nitrosylatable cys residues to the C10 domain of cMyBP-C (Figueiredo-Freitas et al., 2013). A second study of nitrosylation in mouse heart homogenate reports additional cMyBP-C nitrosylation of the $\mathrm{N}$-terminal region at 2 cysteines within the residues that would be encompassed by our C0C3 fragment (Kohr et al., 2011). We suspect the discrepancy between their and our findings in N-terminal nitrosylation results from the sensitivity of mass spectrometry used in their study; its ability to detect nitrosylation in a small fraction of molecules does not evidence a significant level of nitrosylation.

Nitrosylation of cMyBP-C also had no functional effect on its ability to bind to LMM in vitro. Domain C10 binds directly with the LMM portion of the myosin rod through charged ionic interactions at isoform-specific stoichiometries (Alyonycheva et al., 1997; Okagaki et al., 2007). It is essential for myosin rod binding, though it is not sufficient for correct sarcomeric incorporation (Gilbert et al., 1996). cMyBP-C domains C7-C9 are required for localization of cMyBP-C to the C-zone of the A-band. These domains interact with titin and other thick filament associated proteins, and it is these associations that are thought to 
determine its specific localization. Interestingly, myosin binding protein-H (MyBP-H) has a C-terminal domain homologous to cMyBP-C C10 and is shown to competitively bind to LMM (Alyonycheva et al., 1997); however, MyBP-H appears to lack the same titin-binding ability (Soteriou et al., 1993) and exhibits a different sarcomeric incorporation pattern than cMyBP-C (Alyonycheva et al., 1997).

The ability to nitrosylate a protein in vitro does not evidence its endogenous nitrosylation nor does protein nitrosylation invariably affect protein function. Unpublished data from our lab suggest that nitrosylation of sarcomeric proteins actin and $\alpha$-actinin does not have large effects on protein function. cMyBP-C nitrosylation also does not appear to play a functional role in crossbridge regulation.

\subsection{Conclusion}

This inaugural investigation of the functional effect of cMyBP-C nitrosylation refutes the hypothesis proposed in Aim 2B, suggesting that nitrosylation does not play a role in modulating the ability of cMyBP-C to regulate crossbridge function nor does it affect cMyBP-C binding to the myosin thick filament backbone. These data suggest that responsibility for the depressed cardiac contractility associated with myofibrillar nitrosylation lies in another of the nitrosylatable sarcomeric proteins. 
Chapter 5: Conclusions and

Future Directions 


\subsection{Implications for muscle contraction}

The work presented here integrates our understanding of the striated muscle sarcomere with a more detailed knowledge of the bond interfaces, binding partners, functions, and regulation of key crossbridge proteins. In Chapter 2 we showed that loop 2 of myosin is a force-dependent inhibitor of the rigor bond. Based on our measured biomechanical detachment rate of the rigor actomyosin crossbridge, we were able to predict the effect that this force-dependent dissociation pathway might have on the unloaded shortening velocity and the duty ratio in muscle fibers.

Actomyosin crossbridge detachment occurs through ATP binding to the myosin head and causes a conformational change that releases the bound actin filament. The rapid detachment rate in an ATP-rich environment generally precludes myosin detachment in other nucleotide states. However, the load-dependent behavior of the actomyosin bond (Guo and Guilford, 2006; Rao et al., 2009) suggests that biochemical detachment upon ATP binding may not be the only mechanism at play. In our models of smooth and skeletal muscle time to detachment we found that mechanical actin filament detachment from nucleotide-free or ADP-bound myosin might be a competing mechanism that augments crossbridge turnover over a select range of forces. Indeed, we saw that the skeletal crossbridge detachment rate was greater than that of biochemical ATP-dependent dissociation with applied forces up to $2 \mathrm{pN}$ at a physiologic ATP concentration. Presumably the smooth muscle actomyosin bond lifetime would respond similarly to force and exhibit catch bond behavior. If we apply this assumption to our model along with experimentallydetermined smooth muscle biochemical detachment rates for the remaining steps, the predicted effect is that mechanical unbinding plays an even greater role in accelerating smooth muscle detachment over a broader range of forces.

The role of cMyBP-C in crossbridge regulation remains an important area of inquiry. In vitro cMyBP-C inhibits actomyosin motility (Razumova et al., 2006; Saber et al., 2008). cMyBP-C phosphorylation relieves this inhibition (Weith et al., 2012a). We provide evidence that refutes the prominent hypothesis by which cMyBP-C is thought to regulate 
the actomyosin crossbridge - that cMyBP-C N-terminal binding to either actin or to myosin imposes a physical drag on the protein and is directly responsible for inhibiting its motion, and that phosphorylation alleviates this drag by reducing cMyBP-C binding. In Chapter 3 we directly measured the bond between actin and N-terminal cMyBP-C and found it was unresponsive to $\mathrm{cMyBP}-\mathrm{C}$ phosphorylation. The same was true for the bond between cMyBP-C and the myosin head. Because neither bond was responsive to cMyBP-C phosphorylation, we conclude that neither a bond with actin nor with myosin could possibly explain a phosphorylation-dependent drag imposed by cMyBP-C on one of these proteins. This significantly narrows the possibilities by which the system may be regulated. Clearly the means by which cMyBP-C modulates actomyosin kinetics needs to be reexamined with a new mechanism of interaction with the crossbridge in mind.

Nitrosylation proved not to be a novel modulator of cMyBP-C function in our hands. Despite cMyBP-C's ability to be nitrosylated, neither LMM binding nor regulation of crossbridge cycling were functionally affected. Presumably cMyBP-C nitrosylation would have similar inactivity in vivo, however it is possible that its effects are sensitive to the physiological environment and the ensemble of proteins present in the sarcomere.

\subsection{Future directions}

\section{Loop 2 load-dependent regulation of the actomyosin crossbridge}

Our loop 2 experiments were conducted with a mixed-isoform population of rat skeletal HMM. However, there is substantial evidence that the myosin family- and isoform-specific identity of loop 2 directly tunes its kinetic activity. The loop 2 amino acid sequence is poorly conserved between different myosin families, yet when compared between myosins that are kinetically similar the sequence is actually more constrained than that of the rest of the myosin molecule (Goodson et al., 1999). The characteristics of myosin heads with loop 2 chimeric substitutions reflected the activity of the donor myosins when actin-activated ATPase was measured (Uyeda et al., 1994). These data suggest that the large variations in length and sequence of loop 2 may fine-tune the activity of each myosin isoform. 
To get a more complete measure of the role that myosin loop 2 plays as a load-dependent regulator of the actomyosin crossbridge, we need to perform our bond mechanics experiments using other myosin isoforms. The load-dependent behavior observed by our group with skeletal muscle myosin has not been tested in cardiac or smooth muscle. Loop 2 is essential for load-dependence in skeletal myosin, but the structural and biochemical differences may not make this a uniform feature in other isoforms.

\section{cMyBP-C-crossbridge interactions and regulation}

As stated in Chapter 3, the physiological crossbridge binding partner and mechanism of regulation of cMyBP-C are unknown, yet a minimal in vitro motility assay containing only actin and myosin shows it to be inhibitory to actomyosin function. We have directly probed the interaction of N-terminal cMyBP-C with actin and with myosin heads separately and our results cannot explain the inhibitory behavior of cMyBP-C or its relief by phosphorylation.

A laser trap experiment that reflects the flow-cell set up of the in vitro motility assay would allow the load-dependent bond mechanics of inhibition to be reexamined. Myosin would be adsorbed to the nitrocellulose surface, biotinylated actin would be coupled to the lasertrapped microsphere, and cMyBP-C N-terminal fragments would be added in solution and allowed to interact freely as they do in the motility set up. The bond lifetimes measured between the actin- and myosin-coated surfaces would be indicative of any role $\mathrm{N}$-terminal cMyBP-C plays in regulating actomyosin interactions. Further experiments would likely be necessary to clarify its mechanism of inhibition as well as the role of cMyBP-C phosphorylation in modulating this mechanism.

\subsection{Implications for health and disease}

While the power stroke of myosin and nucleotide binding and hydrolysis are reasonably well understood, the molecular-level details of the actin-myosin interactions themselves have not been fully elucidated. Interestingly, the interaction between actin and myosin in the crossbridge is dependent upon the load applied to the bond between them (Guo and Guilford, 2006; Rao et al., 2009). The present work discovered that loop 2 is responsible for 
the load-dependent behavior of the crossbridge and shows how that might be important to a muscle's unloaded shortening velocity. Disruption of this force-dependent behavior makes myosin unable to bind to actin in a long-lived state; counterintuitively, the shortening velocity would decrease and a muscle would become dependent solely upon ATP binding to dissociate the actomyosin crossbridge.

Ultimately, the goal of this study is to aid in understanding the basic mechanisms involved in muscle contraction at the crossbridge-level and to address clinically relevant questions that may be explored in parallel. cMyBP-C mutations are a leading cause of hypertrophic cardiomyopathy and emphasize the importance of cMyBP-C to normal cardiac function, though little is known about the mechanism by which its mutations result in disease. cMyBP-C mutations often manifest in truncated fragments of cMyBP-C, leading to transcripts lacking $\mathrm{N}$-terminal crossbridge binding domains or more commonly lacking the C-terminal domains responsible for thick-filament binding (Carrier et al., 1997). Studies of human patient biopsies and in mouse models have shown that fragments of cMyBP-C may contribute to hypertrophic cardiomyopathy through reduced expression in haploinsufficiency (Dijk et al., 2009; Rottbauer et al., 1997) or they act as dominantnegative alleles where truncated proteins are expressed and incorporated into the sarcomere but disrupt normal function (Flavigny et al., 1999; Harris et al., 2002; Yang et al., 1998). There is also evidence that mutations manifest in varied phosphorylation states of cMyBP-C, suggesting that more than the protein itself, but also its post-translational modifications are critical to maintaining cardiac function (Barefield and Sadayappan, 2010). If we can determine the mechanism of regulation between cMyBP-C and the crossbridge, the mechanism of disease may also become clear. Furthermore, protein engineering could be used to introduce a series of point-mutations to determine the key regulatory mechanism of crossbridge binding and kinetics. This may suggest a therapeutic strategy to improve contractility in disease states such as hypertrophic cardiomyopathy. 


\section{References}

Ababou, A., Gautel, M., and Pfuhl, M. (2007). Dissecting the N-terminal Myosin Binding Site of Human Cardiac Myosin-binding Protein C STRUCTURE AND MYOSIN BINDING OF DOMAIN C2. J. Biol. Chem. 282, 9204-9215.

Ababou, A., Rostkova, E., Mistry, S., Masurier, C.L., Gautel, M., and Pfuhl, M. (2008). Myosin Binding Protein C Positioned to Play a Key Role in Regulation of Muscle Contraction: Structure and Interactions of Domain C1. J. Mol. Biol. 384, 615-630.

Allersma, M.W., Gittes, F., deCastro, M.J., Stewart, R.J., and Schmidt, C.F. (1998). TwoDimensional Tracking of ncd Motility by Back Focal Plane Interferometry. Biophys. J. 74, 1074-1085.

Alon, R., Hammer, D.A., and Springer, T.A. (1995). Lifetime of the P-selectin-carbohydrate bond and its response to tensile force in hydrodynamic flow. Nature 374, 539-542.

Alyonycheva, T.N., Mikawa, T., Reinach, F.C., and Fischman, D.A. (1997). Isoform-specific Interaction of the Myosin-binding Proteins (MyBPs) with Skeletal and Cardiac Myosin Is a Property of the C-terminal Immunoglobulin Domain. J. Biol. Chem. 272, 20866-20872.

Andrade, F.H., Reid, M.B., Allen, D.G., and Westerblad, H. (1998). Effect of nitric oxide on single skeletal muscle fibres from the mouse. J. Physiol. 509, 577-586.

Bagshaw, C.R., Eccleston, J.F., Trentham, D.R., Yates, D.W., and Goody, R.S. (1973). Transient Kinetic Studies of the Mg++-dependent ATPase of Myosin and Its Proteolytic Subfragments. Cold Spring Harb. Symp. Quant. Biol. 37, 127-135.

Bálint, M., Sréter, F.A., Wolf, I., Nagy, B., and Gergely, J. (1975). The substructure of heavy meromyosin. The effect of $\mathrm{Ca} 2+$ and $\mathrm{Mg} 2+$ on the tryptic fragmentation of heavy meromyosin. J. Biol. Chem. 250, 6168 -6177.

Barefield, D., and Sadayappan, S. (2010). Phosphorylation and function of cardiac myosin binding protein-C in health and disease. J. Mol. Cell. Cardiol. 48, 866-875. 
Behrmann, E., Müller, M., Penczek, P.A., Mannherz, H.G., Manstein, D.J., and Raunser, S. (2012). Structure of the Rigor Actin-Tropomyosin-Myosin Complex. Cell 150, 327-338.

Bell, G.I. (1978). Models for the specific adhesion of cells to cells. Science 200, 618-627.

Block, S.M. (1995). Nanometres and piconewtons: the macromolecular mechanics of kinesin. Trends Cell Biol. 5, 169-175.

Bobkov, A.A., Bobkova, E.A., Lin, S.H., and Reisler, E. (1996). The role of surface loops (residues 204-216 and 627-646) in the motor function of the myosin head. Proc. Natl. Acad. Sci. 93, $2285-2289$.

Bonne, G., Carrier, L., Richard, P., Hainque, B., and Schwartz, K. (1998). Familial Hypertrophic Cardiomyopathy From Mutations to Functional Defects. Circ. Res. 83, 580593.

Botts, J., Muhlrad, A., Takashi, R., and Morales, M.F. (1982). Effects of tryptic digestion on myosin subfragment- 1 and its actin-activated ATPase. Biochemistry (Mosc.) 21, 69036905.

Calaghan, S.C., Trinick, J., Knight, P.J., and White, E. (2000). A role for C-protein in the regulation of contraction and intracellular $\mathrm{Ca} 2+$ in intact rat ventricular myocytes. J. Physiol. 528, 151-156.

Capitanio, M., Canepari, M., Cacciafesta, P., Lombardi, V., Cicchi, R., Maffei, M., Pavone, F.S., and Bottinelli, R. (2006). Two independent mechanical events in the interaction cycle of skeletal muscle myosin with actin. Proc. Natl. Acad. Sci. U. S. A. 103, 87-92.

Carrier, L., Bonne, G., Bahrend, E., Yu, B., Richard, P., Niel, F., Hainque, B., Cruaud, C., Gary, F., Labeit, S., et al. (1997). Organization and Sequence of Human Cardiac Myosin Binding Protein C Gene (MYBPC3) and Identification of Mutations Predicted to Produce Truncated Proteins in Familial Hypertrophic Cardiomyopathy. Circ. Res. 80, 427-434. 
Chen, W., Evans, E.A., McEver, R.P., and Zhu, C. (2008). Monitoring Receptor-Ligand Interactions between Surfaces by Thermal Fluctuations. Biophys. J. 94, 694-701.

Chesla, S.E., Selvaraj, P., and Zhu, C. (1998). Measuring Two-Dimensional Receptor-Ligand Binding Kinetics by Micropipette. Biophys. J. 75, 1553-1572.

Cluzel, P., Lebrun, A., Heller, C., Lavery, R., Viovy, J.L., Chatenay, D., and Caron, F. (1996). DNA: an extensible molecule. Science 271, 792-794.

Coates, J.H., Criddle, A.H., and Geeves, M.A. (1985). Pressure-relaxation studies of pyrenelabelled actin and myosin subfragment 1 from rabbit skeletal muscle. Evidence for two states of acto-subfragment 1. Biochem. J. 232, 351-356.

Copeland, O., Sadayappan, S., Messer, A.E., Steinen, G.J.M., van der Velden, J., and Marston, S.B. (2010). Analysis of cardiac myosin binding protein-C phosphorylation in human heart muscle. J. Mol. Cell. Cardiol. 49, 1003-1011.

Cremo, C.R., and Geeves, M.A. (1998). Interaction of Actin and ADP with the Head Domain of Smooth Muscle Myosin: Implications for Strain-Dependent ADP Release in Smooth Muscle†. Biochemistry (Mosc.) 37, 1969-1978.

Debold, E.P., Patlak, J.B., and Warshaw, D.M. (2005). Slip Sliding Away: Load-Dependence of Velocity Generated by Skeletal Muscle Myosin Molecules in the Laser Trap. Biophys. J. 89, L34-L36.

Dembo, M., Torney, D.C., Saxman, K., and Hammer, D. (1988). The Reaction-Limited Kinetics of Membrane-to-Surface Adhesion and Detachment. Proc. R. Soc. Lond. B Biol. Sci. 234, 5583.

Dijk, S.J. van, Dooijes, D., Remedios, C. dos, Michels, M., Lamers, J.M.J., Winegrad, S., Schlossarek, S., Carrier, L., Cate, F.J. ten, Stienen, G.J.M., et al. (2009). Cardiac MyosinBinding Protein C Mutations and Hypertrophic Cardiomyopathy Haploinsufficiency, Deranged Phosphorylation, and Cardiomyocyte Dysfunction. Circulation 119, 1473-1483. 
Dupuis, D., William H. Guilford, Wu, J., and Warshaw, D. (1997). Actin filament mechanics in the laser trap. J. Muscle Res. Cell Motil. 18, 17-30.

Eisenberg, E., and Moos, C. (1968). Adenosinetriphosphatase activity of acto-heavy meromyosin. Kinetic analysis of actin activation. Biochemistry (Mosc.) 7, 1486-1489.

Evangelista, A.M., Rao, V.S., Filo, A.R., Marozkina, N.V., Doctor, A., Jones, D.R., Gaston, B., and Guilford, W.H. (2010). Direct Regulation of Striated Muscle Myosins by Nitric Oxide and Endogenous Nitrosothiols. PLoS ONE 5, e11209.

Figueiredo - Freitas, C., Foster, M.W., Nogueira, L., Liang, J., Yamashita, A., Dulce, R., Thompson, J.W., Hare, J.M., Moseley, M.A., Sorenson, M.M., et al. (2013). S-nitrosylation decreases Ca2+ sensitivity and actomyosin ATPase activity of contractile proteins in cardiac myofibrils. 2013 Biophys. Soc. Meet. Abstr. Biophys. J. 2309-Pos.

Finer, J.T., Simmons, R.M., and Spudich, J.A. (1994). Single myosin molecule mechanics: piconewton forces and nanometre steps. Nature 368, 113-119.

Flavigny, J., Souchet, M., Sébillon, P., Berrebi-Bertrand, I., Hainque, B., Mallet, A., Bril, A., Schwartz, K., and Carrier, L. (1999). COOH-terminal truncated cardiac myosin-binding protein $\mathrm{C}$ mutants resulting from familial hypertrophic cardiomyopathy mutations exhibit altered expression and/or incorporation in fetal rat cardiomyocytes. J. Mol. Biol. 294, 443456.

Fraterman, S., Zeiger, U., Khurana, T.S., Wilm, M., and Rubinstein, N.A. (2007). Quantitative Proteomics Profiling of Sarcomere Associated Proteins in Limb and Extraocular Muscle Allotypes. Mol. Cell. Proteomics 6, 728-737.

Fritz, J., Katopodis, A.G., Kolbinger, F., and Anselmetti, D. (1998). Force-mediated kinetics of single P-selectin/ligand complexes observed by atomic force microscopy. Proc. Natl. Acad. Sci. 95, 12283-12288.

Galler, S., Hilber, K., and Göbesberger, A. (1997). Effects of nitric oxide on force-generating proteins of skeletal muscle. Eur. J. Physiol. 434, 242-245. 
Gautel, M., Zuffardi, O., Freiburg, A., and Labeit, S. (1995). Phosphorylation switches specific for the cardiac isoform of myosin binding protein-C: a modulator of cardiac contraction? EMBO J. 14, 1952-1960.

Geeves, M.A., and Conibear, P.B. (1995). The role of three-state docking of myosin S1 with actin in force generation. Biophys. J. 68, 194S-201S.

Geeves, M.A., and Holmes, K.C. (2005). The Molecular Mechanism of Muscle Contraction. In Advances in Protein Chemistry, and D.A.D.P. John M. Squire, ed. (Academic Press), pp. 161193.

Geeves, M.A., Goody, R.S., and Gutfreund, H. (1984). Kinetics of acto-S1 interaction as a guide to a model for the crossbridge cycle. J. Muscle Res. Cell Motil. 5, 351-361.

Gilbert, R., Kelly, M.G., Mikawa, T., and Fischman, D.A. (1996). The carboxyl terminus of myosin binding protein C (MyBP-C, C-protein) specifies incorporation into the A-band of striated muscle. J. Cell Sci. 109, 101-111.

Goodson, H.V., Warrick, H.M., and Spudich, J.A. (1999). Specialized conservation of surface loops of myosin: evidence that loops are involved in determining functional characteristics. J. Mol. Biol. 287, 173-185.

Gränicher, D., and Portzehl, H. (1964). The influence of magnesium and calcium pyrophosphate chelates, of free magnesium ions, free calcium ions, and free pyrophosphate ions on the dissociation of actomyosin in solution. Biochim. Biophys. Acta BBA - Gen. Subj. $86,567-578$.

Gruen, M., and Gautel, M. (1999). Mutations in $\beta$-myosin S2 that cause familial hypertrophic cardiomyopathy (FHC) abolish the interaction with the regulatory domain of myosinbinding protein-C. J. Mol. Biol. 286, 933-949.

Gruen, M., Prinz, H., and Gautel, M. (1999). cAPK-phosphorylation controls the interaction of the regulatory domain of cardiac myosin binding protein C with myosin-S2 in an on-off fashion. FEBS Lett. 453, 254-259. 
Guilford, W.H., and Filo, A.R. (2010). The Nitrosoproteome of Skeletal and Cardiac Myofibrils. Free Radic. Biol. Med. 49, S114.

Guilford, W.H., and Warshaw, D.M. (1998). The molecular mechanics of smooth muscle myosin. Comp. Biochem. Physiol. B Biochem. Mol. Biol. 119, 451-458.

Guilford, W.H., Dupuis, D.E., Kennedy, G., Wu, J., Patlak, J.B., and Warshaw, D.M. (1997). Smooth muscle and skeletal muscle myosins produce similar unitary forces and displacements in the laser trap. Biophys. J. 72, 1006-1021.

Guilford, W.H., Tournas, J.A., Dascalu, D., and Watson, D.S. (2004). Creating multiple timeshared laser traps with simultaneous displacement detection using digital signal processing hardware. Anal. Biochem. 326, 153-166.

Guo, B., and Guilford, W.H. (2004). The tail of myosin reduces actin filament velocity in the in vitro motility assay. Cell Motil. Cytoskeleton 59, 264-272.

Guo, B., and Guilford, W.H. (2006). Mechanics of actomyosin bonds in different nucleotide states are tuned to muscle contraction. Proc. Natl. Acad. Sci. 103, 9844 -9849.

Han, P., Zhou, X., Huang, B., Zhang, X., and Chen, C. (2008). On-gel fluorescent visualization and the site identification of S-nitrosylated proteins. Anal. Biochem. 377, 150-155.

Harris, D.E., and Warshaw, D.M. (1993). Smooth and skeletal muscle myosin both exhibit low duty cycles at zero load in vitro. J. Biol. Chem. 268, 14764-14768.

Harris, S.P., Bartley, C.R., Hacker, T.A., McDonald, K.S., Douglas, P.S., Greaser, M.L., Powers, P.A., and Moss, R.L. (2002). Hypertrophic Cardiomyopathy in Cardiac Myosin Binding Protein-C Knockout Mice. Circ. Res. 90, 594-601.

Harris, S.P., Lyons, R.G., and Bezold, K.L. (2011). In the Thick of It HCM-Causing Mutations in Myosin Binding Proteins of the Thick Filament. Circ. Res. 108, 751-764.

Hartzell, H.C. (1985). Effects of phosphorylated and unphosphorylated C-protein on cardiac actomyosin ATPase. J. Mol. Biol. 186, 185-195. 
Herrera, A.M., Kuo, K.-H., and Seow, C.Y. (2002). Influence of calcium on myosin thick filament formation in intact airway smooth muscle. Am. J. Physiol. - Cell Physiol. 282, C310C316.

Herron, T.J. (2006). Activation of Myocardial Contraction by the N-Terminal Domains of Myosin Binding Protein-C. Circ. Res. 98, 1290-1298.

Hill, A.V. (1938). The Heat of Shortening and the Dynamic Constants of Muscle. Proc. R. Soc. Lond. B Biol. Sci. 126, 136-195.

Hofmann, P.A., Hartzell, H.C., and Moss, R.L. (1991a). Alterations in Ca2+ sensitive tension due to partial extraction of C-protein from rat skinned cardiac myocytes and rabbit skeletal muscle fibers. J. Gen. Physiol. 97, 1141-1163.

Hofmann, P.A., Greaser, M.L., and Moss, R.L. (1991b). C-protein limits shortening velocity of rabbit skeletal muscle fibres at low levels of Ca2+ activation. J. Physiol. 439, 701-715.

Hooft, A.M., Maki, E.J., Cox, K.K., and Baker, J.E. (2007). An Accelerated State of MyosinBased Actin Motility†. Biochemistry (Mosc.) 46, 3513-3520.

Howard, J. (2001). Mechanics of motor proteins and the cytoskeleton (Sunderland, Mass.: Sinauer Associates, Publishers).

Inoue, Y., and Adachi, T. (2013). Role of the Actin-Myosin Catch Bond on Actomyosin Aggregate Formation. Cell. Mol. Bioeng. 6, 3-12.

Jacques, A.M., Copeland, O., Messer, A.E., Gallon, C.E., King, K., McKenna, W.J., Tsang, V.T., and Marston, S.B. (2008). Myosin binding protein C phosphorylation in normal, hypertrophic and failing human heart muscle. J. Mol. Cell. Cardiol. 45, 209-216.

Jaffrey, S.R., and Snyder, S.H. (2001). The Biotin Switch Method for the Detection of SNitrosylated Proteins. Sci. Signal. 2001, pl1. 
Jia, W., Shaffer, J.F., Harris, S.P., and Leary, J.A. (2010). Identification of Novel Protein Kinase A Phosphorylation Sites in the M-domain of Human and Murine Cardiac Myosin Binding Protein-C Using Mass Spectrometry Analysis. J. Proteome Res. 9, 1843-1853.

Karsai, Á., Kellermayer, M.S.Z., and Harris, S.P. (2011). Mechanical Unfolding of Cardiac Myosin Binding Protein-C by Atomic Force Microscopy. Biophys. J. 101, 1968-1977.

Karsai, Á., Kellermayer, M.S.Z., and Harris, S.P. (2013). Cross-Species Mechanical Fingerprinting of Cardiac Myosin Binding Protein-C. Biophys. J. 104, 2465-2475.

Khromov, A.S., Somlyo, A.V., and Somlyo, A.P. (1996). Nucleotide binding by actomyosin as a determinant of relaxation kinetics of rabbit phasic and tonic smooth muscle. J. Physiol. $492,669-673$.

Kitamura, K., Tokunaga, M., Iwane, A.H., and Yanagida, T. (1999). A single myosin head moves along an actin filament with regular steps of 5.3 nanometres. Nature 397, 129-134.

Kohr, M.J., Aponte, A.M., Sun, J., Wang, G., Murphy, E., Gucek, M., and Steenbergen, C. (2011). Characterization of potential S-nitrosylation sites in the myocardium. Am. J. Physiol. - Heart Circ. Physiol. 300, H1327-H1335.

Kong, F., García, A.J., Mould, A.P., Humphries, M.J., and Zhu, C. (2009). Demonstration of catch bonds between an integrin and its ligand. J. Cell Biol. 185, 1275-1284.

Korn, E.D. (2000). Coevolution of head, neck, and tail domains of myosin heavy chains. Proc. Natl. Acad. Sci. 97, 12559-12564.

Kron, S.J., Toyoshima, Y.Y., Uyeda, T.Q.P., and Spudich, J.A. (1991). [33] Assays for actin sliding movement over myosin-coated surfaces. In Methods in Enzymology, Richard B. Vallee, ed. (Academic Press), pp. 399-416.

Kulikovskaya, I., McClellan, G., Flavigny, J., Carrier, L., and Winegrad, S. (2003). Effect of MyBP-C Binding to Actin on Contractility in Heart Muscle. J. Gen. Physiol. 122, 761-774. 
Kunst, G., Kress, K.R., Gruen, M., Uttenweiler, D., Gautel, M., and Fink, R.H.A. (2000). Myosin Binding Protein C, a Phosphorylation-Dependent Force Regulator in Muscle That Controls the Attachment of Myosin Heads by Its Interaction With Myosin S2. Circ. Res. 86, 51-58.

Linari, M., Dobbie, I., Reconditi, M., Koubassova, N., Irving, M., Piazzesi, G., and Lombardi, V. (1998). The stiffness of skeletal muscle in isometric contraction and rigor: the fraction of myosin heads bound to actin. Biophys. J. 74, 2459-2473.

Liu, Y., Scolari, M., Im, W., and Woo, H. (2006). Protein-protein interactions in actin-myosin binding and structural effects of R405Q mutation: A molecular dynamics study. Proteins Struct. Funct. Bioinforma. 64, 156-166.

Lorenz, M., and Holmes, K.C. (2010). The actin-myosin interface. Proc. Natl. Acad. Sci. 107, $12529-12534$.

Lu, Y., Kwan, A.H., Trewhella, J., and Jeffries, C.M. (2011). The C0C1 Fragment of Human Cardiac Myosin Binding Protein C Has Common Binding Determinants for Both Actin and Myosin. J. Mol. Biol. 413, 908-913.

Luther, P.K., Winkler, H., Taylor, K., Zoghbi, M.E., Craig, R., Padrón, R., Squire, J.M., and Liu, J. (2011). Direct visualization of myosin-binding protein $C$ bridging myosin and actin filaments in intact muscle. Proc. Natl. Acad. Sci. 108, 11423-11428.

Margossian, S.S., and Lowey, S. (1982). Preparation of myosin and its subfragments from rabbit skeletal muscle. Methods Enzymol. 85 Pt B, 55-71.

Marshall, B.T., Long, M., Piper, J.W., Yago, T., McEver, R.P., and Zhu, C. (2003). Direct observation of catch bonds involving cell-adhesion molecules. Nature 423, 190-193.

Marston, S.B. (1982). The rates of formation and dissociation of actin-myosin complexes. Effects of solvent, temperature, nucleotide binding and head-head interactions. Biochem. J. 203, 453-460. 
McConnell, B.K., Jones, K.A., Fatkin, D., Arroyo, L.H., Lee, R.T., Aristizabal, O., Turnbull, D.H., Georgakopoulos, D., Kass, D., Bond, M., et al. (1999). Dilated cardiomyopathy in homozygous myosin-binding protein-C mutant mice. J. Clin. Invest. 104, 1235-1244.

Merkel, R., Nassoy, P., Leung, A., Ritchie, K., and Evans, E. (1999). Energy landscapes of receptor-ligand bonds explored with dynamic force spectroscopy. Nature 397, 50-53.

Michalek, A.J., Howarth, J.W., Gulick, J., Previs, M.J., Robbins, J., Rosevear, P.R., and Warshaw, D.M. (2013). Phosphorylation Modulates the Mechanical Stability of the Cardiac MyosinBinding Protein C Motif. Biophys. J. 104, 442-452.

Millar, N.C., and Geeves, M.A. (1988). Protein fluorescence changes associated with ATP and adenosine 5'-[gamma-thio]triphosphate binding to skeletal muscle myosin subfragment 1 and actomyosin subfragment 1. Biochem. J. 249, 735-743.

Miyamoto, C.A., Fischman, D.A., and Reinach, F.C. (1999). The interface between MyBP-C and myosin: site-directed mutagenesis of the CX myosin-binding domain of MyBP-C. J. Muscle Res. Cell Motil. 20, 703-716.

Mocz, G., Szilagyi, L., Chen Lu, R., Fabian, F., Balint, M., and Gergely, J. (1984). Effect of nucleotides, divalent cations and temperature on the tryptic susceptibility of myosin subfragment 1. Eur. J. Biochem. 145, 221-229.

Molloy, J.E., Burns, J.E., Kendrick-Jones, J., Tregear, R.T., and White, D.C.S. (1995). Movement and force produced by a single myosin head. Nature 378, 209-212.

Moos, C., Offer, G., Starr, R., and Bennett, P. (1975). Interaction of C-protein with myosin, myosin rod and light meromyosin. J. Mol. Biol. 97, 1-9.

Mornet, D., Bertrand, R., Pantel, P., Audemard, E., and Kassab, R. (1981). Proteolytic approach to structure and function of actin recognition site in myosin heads. Biochemistry (Mosc.) 20, 2110-2120. 
Mun, J.Y., Previs, M.J., Yu, H.Y., Gulick, J., Tobacman, L.S., Previs, S.B., Robbins, J., Warshaw, D.M., and Craig, R. (2014). Myosin-binding protein C displaces tropomyosin to activate cardiac thin filaments and governs their speed by an independent mechanism. Proc. Natl. Acad. Sci. 201316001.

Murphy, C.T., and Spudich, J.A. (2000). Variable surface loops and myosin activity: Accessories to a motor. J. Muscle Res. Cell Motil. 21, 139-151.

Okagaki, T., Suzuki, R., and Ooi, A. (2007). C-protein (MyBP-C) isoforms from carp ordinary and dark muscles and muscle type-specific binding to myosin. Fish. Sci. 73, 640-650.

Pardee, J., and Spudich, J. (1982). Purification of muscle actin. Methods Enzymol. 85 Pt B, 164-181.

Pereverzev, Y.V., Prezhdo, O.V., Forero, M., Sokurenko, E.V., and Thomas, W.E. (2005). The Two-Pathway Model for the Catch-Slip Transition in Biological Adhesion. Biophys. J. 89, 1446-1454.

Perissinotti, L.L., Turjanski, A.G., Estrin, D.A., and Doctorovich, F. (2005). Transnitrosation of Nitrosothiols: Characterization of an Elusive Intermediate. J. Am. Chem. Soc. 127, 486487.

Perkins, W.J., Han, Y.-S., and Sieck, G.C. (1997). Skeletal muscle force and actomyosin ATPase activity reduced by nitric oxide donor. J. Appl. Physiol. 83, 1326-1332.

Pfuhl, M., and Gautel, M. (2012). Structure, interactions and function of the N-terminus of cardiac myosin binding protein C (MyBP-C): who does what, with what, and to whom? J. Muscle Res. Cell Motil. 33, 83-94.

Phan, U.T., Waldron, T.T., and Springer, T.A. (2006). Remodeling of the lectin-EGF-like domain interface in $\mathrm{P}$ - and L-selectin increases adhesiveness and shear resistance under hydrodynamic force. Nat. Immunol. 7, 883-889. 
Pierres, A., Benoliel, A.M., Bongrand, P., and van der Merwe, P.A. (1996). Determination of the lifetime and force dependence of interactions of single bonds between surface-attached CD2 and CD48 adhesion molecules. Proc. Natl. Acad. Sci. 93, 15114-15118.

Prezhdo, O.V., and Pereverzev, Y.V. (2009). Theoretical Aspects of the Biological Catch Bond. Acc. Chem. Res. 42, 693-703.

Rao, V.S., Marongelli, E.N., and Guilford, W.H. (2009). Phosphorylation of Tropomyosin Extends Cooperative Binding of Myosin Beyond a Single Regulatory Unit. Cell Motil. Cytoskeleton 66, 10-23.

Rao, V.S., Clobes, A.M., and Guilford, W.H. (2011). Force Spectroscopy Reveals Multiple "Closed States" of the Muscle Thin Filament. J. Biol. Chem. 286, 24135 -24141.

Rayment, I., Holden, H.M., Whittaker, M., Yohn, C.B., Lorenz, M., Holmes, K.C., and Milligan, R.A. (1993a). Structure of the actin-myosin complex and its implications for muscle contraction. Science 261, 58-65.

Rayment, I., Rypniewski, W.R., Schmidt-Base, K., Smith, R., Tomchick, D.R., Benning, M.M., Winkelmann, D.A., Wesenberg, G., and Holden, H.M. (1993b). Three-dimensional structure of myosin subfragment-1: a molecular motor. Science 261, 50-58.

Razumova, M.V., Shaffer, J.F., Tu, A.-Y., Flint, G.V., Regnier, M., and Harris, S.P. (2006). Effects of the N-terminal Domains of Myosin Binding Protein-C in an in Vitro Motility Assay EVIDENCE FOR LONG-LIVED CROSS-BRIDGES. J. Biol. Chem. 281, 35846-35854.

Rief, M., Gautel, M., Oesterhelt, F., Fernandez, J.M., and Gaub, H.E. (1997). Reversible unfolding of individual titin immunoglobulin domains by AFM. Science 276, 1109-1112.

Rinko, L.J., Lawrence, M.B., and Guilford, W.H. (2004). The Molecular Mechanics of P- and LSelectin Lectin Domains Binding to PSGL-1. Biophys. J. 86, 544.

Ross, S.M. (2009). Introduction to Probability and Statistics for Engineers and Scientists (Academic Press). 
Rottbauer, W., Gautel, M., Zehelein, J., Labeit, S., Franz, W.M., Fischer, C., Vollrath, B., Mall, G., Dietz, R., Kubler, W., et al. (1997). Novel splice donor site mutation in the cardiac myosinbinding protein-C gene in familial hypertrophic cardiomyopathy. Characterization of cardiac transcript and protein. J. Clin. Invest. 100, 475-482.

Rybakova, I.N., Greaser, M.L., and Moss, R.L. (2011). Myosin Binding Protein C Interaction with Actin CHARACTERIZATION AND MAPPING OF THE BINDING SITE. J. Biol. Chem. 286, 2008-2016.

Saber, W., Begin, K.J., Warshaw, D.M., and VanBuren, P. (2008). Cardiac myosin binding protein-C modulates actomyosin binding and kinetics in the in vitro motility assay. J. Mol. Cell. Cardiol. 44, 1053-1061.

Sadayappan, S., Gulick, J., Osinska, H., Martin, L.A., Hahn, H.S., Dorn, G.W., Klevitsky, R., Seidman, C.E., Seidman, J.G., and Robbins, J. (2005). Cardiac Myosin-Binding Protein-C Phosphorylation and Cardiac Function. Circ. Res. 97, 1156-1163.

Sadayappan, S., Osinska, H., Klevitsky, R., Lorenz, J.N., Sargent, M., Molkentin, J.D., Seidman, C.E., Seidman, J.G., and Robbins, J. (2006). Cardiac myosin binding protein c phosphorylation is cardioprotective. Proc. Natl. Acad. Sci. 103, 16918-16923.

Sadayappan, S., Gulick, J., Klevitsky, R., Lorenz, J.N., Sargent, M., Molkentin, J.D., and Robbins, J. (2009). Cardiac Myosin Binding Protein-C Phosphorylation in a $\beta$-Myosin Heavy Chain Background. Circulation 119, 1253-1262.

Saito, K., Aoki, T., Aoki, T., and Yanagida, T. (1994). Movement of single myosin filaments and myosin step size on an actin filament suspended in solution by a laser trap. Biophys. J. 66, 769-777.

Sarangapani, K.K., Yago, T., Klopocki, A.G., Lawrence, M.B., Fieger, C.B., Rosen, S.D., McEver, R.P., and Zhu, C. (2004). Low Force Decelerates L-selectin Dissociation from P-selectin Glycoprotein Ligand-1 and Endoglycan. J. Biol. Chem. 279, 2291-2298. 
Shaffer, J.F., Kensler, R.W., and Harris, S.P. (2009). The Myosin-binding Protein C Motif Binds to F-actin in a Phosphorylation-sensitive Manner. J. Biol. Chem. 284, 12318-12327.

Shoji, H., Takahashi, S., and Okabe, E. (1999). Intracellular effects of nitric oxide on force production and Ca2+ sensitivity of cardiac myofilaments. Antioxid. Redox Signal. 1, 509521.

Snook, J.H., and Guilford, W.H. (2010). The Effects of Load on E-Selectin Bond Rupture and Bond Formation. Cell. Mol. Bioeng. 3, 128-138.

Snook, J.H., and Guilford, W.H. (2012). A High-Throughput Technique Reveals the Load- and Site Density-Dependent Kinetics of E-Selectin. Cell. Mol. Bioeng. 5, 493-503.

Snook, J.H., Li, J., Helmke, B.P., and Guilford, W.H. (2008). Peroxynitrite inhibits myofibrillar protein function in an in vitro assay of motility. Free Radic. Biol. Med. 44, 14-23.

Soteriou, A., Gamage, M., and Trinick, J. (1993). A survey of interactions made by the giant protein titin. J. Cell Sci. 104, 119-123.

Squire, J.M., Luther, P.K., and Knupp, C. (2003). Structural Evidence for the Interaction of Cprotein (MyBP-C) with Actin and Sequence Identification of a Possible Actin-binding Domain. J. Mol. Biol. 331, 713-724.

Sutoh, K. (1982). An actin-binding site on the 20K fragment of myosin subfragment 1. Biochemistry (Mosc.) 21, 4800-4804.

Svoboda, K., and Block, S.M. (1994). Biological Applications of Optical Forces. Annu. Rev. Biophys. Biomol. Struct. 23, 247-285.

Takagi, Y., Homsher, E.E., Goldman, Y.E., and Shuman, H. (2006). Force Generation in Single Conventional Actomyosin Complexes under High Dynamic Load. Biophys. J. 90, 1295-1307.

Takiguchi, K., Hayashi, H., Kurimoto, E., and Higasshi-Fujime, S. (1990). In Vitro Motility of Skeletal Muscle Myosin and Its Proteolytic Fragments. J. Biochem. (Tokyo) 107, 671-679. 
Taylor, E.W. (1991). Kinetic Studies on the Association and Dissociation of Myosin Subfragment 1 and Actin. J. Biol. Chem. 266, 294-302.

Thomas, W., Forero, M., Yakovenko, O., Nilsson, L., Vicini, P., Sokurenko, E., and Vogel, V. (2006). Catch-Bond Model Derived from Allostery Explains Force-Activated Bacterial Adhesion. Biophys. J. 90, 753-764.

Thomas, W.E., Vogel, V., and Sokurenko, E. (2008). Biophysics of Catch Bonds. Annu. Rev. Biophys. 37, 399-416.

Trentham, D.R., Bardsley, R.G., Eccleston, J.F., and Weeds, A.G. (1972). Elementary processes of the magnesium ion-dependent adenosine triphosphatase activity of heavy meromysin. A transient kinetic approach to the study of kinases and adenosine triphosphatases and a colorimetric inorganic phosphate assay in situ. Biochem. J. 126, 635644.

Tyska, M.J., Dupuis, D.E., Guilford, W.H., Patlak, J.B., Waller, G.S., Trybus, K.M., Warshaw, D.M., and Lowey, S. (1999). Two heads of myosin are better than one for generating force and motion. Proc. Natl. Acad. Sci. 96, 4402-4407.

Uyeda, T.Q.P., Kron, S.J., and Spudich, J.A. (1990). Myosin step size: Estimation from slow sliding movement of actin over low densities of heavy meromyosin. J. Mol. Biol. 214, 699710.

Uyeda, T.Q.P., Warrick, H.M., Kron, S.J., and Spudich, J.A. (1991). Quantized velocities at low myosin densities in an in vitro motility. Nature 352, 307-311.

Uyeda, T.Q.P., Ruppel, K.M., and Spudich, J.A. (1994). Enzymatic activities correlate with chimaeric substitutions at the actin-binding face of myosin. Nature 368, 567-569.

Veigel, C., Molloy, J.E., Schmitz, S., and Kendrick-Jones, J. (2003). Load-dependent kinetics of force production by smooth muscle myosin measured with optical tweezers. Nat. Cell Biol. 5, 980-986. 
Warshaw, D.M., Desrosiers, J.M., Work, S.S., and Trybus, K.M. (1990). Smooth muscle myosin cross-bridge interactions modulate actin filament sliding velocity in vitro. J. Cell Biol. 111, 453-463.

Weith, A., Sadayappan, S., Gulick, J., Previs, M.J., VanBuren, P., Robbins, J., and Warshaw, D.M. (2012a). Unique single molecule binding of cardiac myosin binding protein-C to actin and phosphorylation-dependent inhibition of actomyosin motility requires 17 amino acids of the motif domain. J. Mol. Cell. Cardiol. 52, 219-227.

Weith, A.E., Previs, M.J., Hoeprich, G.J., Previs, S.B., Gulick, J., Robbins, J., and Warshaw, D.M. (2012b). The extent of cardiac myosin binding protein-C phosphorylation modulates actomyosin function in a graded manner. J. Muscle Res. Cell Motil. 33, 449-459.

White, H.D., and Taylor, E.W. (1976). Energetics and mechanism of actomyosin adenosine triphosphatase. Biochemistry (Mosc.) 15, 5818-5826.

Whitten, A.E., Jeffries, C.M., Harris, S.P., and Trewhella, J. (2008). Cardiac myosin-binding protein C decorates F-actin: Implications for cardiac function. Proc. Natl. Acad. Sci. 105, 18360-18365.

Yamamoto, K., and Moos, C. (1983). The C-proteins of rabbit red, white, and cardiac muscles. J. Biol. Chem. 258, 8395-8401.

Yang, Q., Sanbe, A., Osinska, H., Hewett, T.E., Klevitsky, R., and Robbins, J. (1998). A mouse model of myosin binding protein $\mathrm{C}$ human familial hypertrophic cardiomyopathy. J. Clin. Invest. 102, 1292-1300. 


\section{Appendix 1: Extended Methods}




\section{A1.1 Proteins}

\section{HMM purification}

HMM was purified from rat skeletal muscle as described in Guo and Guilford (Guo and Guilford, 2004) with minor modifications. Briefly, $400 \mathrm{mg}$ muscle tissue was homogenized on ice in $2 \mathrm{ml}$ myosin extraction buffer $\left(0.3 \mathrm{M} \mathrm{KCl}, 0.01 \mathrm{M} \mathrm{HEPES}, 0.01 \mathrm{M} \mathrm{Na}_{4} \mathrm{P}_{2} \mathrm{O}_{7} \bullet 10 \mathrm{H}_{2} \mathrm{O}\right.$, $1 \mathrm{mM} \mathrm{MgCl}_{2}, 0.01 \mathrm{M}$ DTT, $1 \mathrm{mM}$ ATP, pH 6.8) with protease inhibitor (SIGMAFAST ${ }^{\text {TM }}$, SigmaAldrich, St. Louis, MO). The homogenate was stirred for 30 minutes on ice, then clarified at $140,000 \mathrm{~g}$ for 1 hour. Supernatant was diluted with 3 volumes of $1 \mathrm{mM}$ DTT and left undisturbed on ice for 1 hour. Myosin was collected by centrifugation at 15,000g for 20 minutes. Precipitated myosin was dissolved in $200 \mu \mathrm{l}$ storage solution $(0.5 \mathrm{M} \mathrm{KCl}, 0.05 \mathrm{M}$ $\mathrm{KH}_{2} \mathrm{PO}_{4}, 2 \mathrm{mM} \mathrm{MgCl}$, $0.01 \mathrm{M} \mathrm{DTT}, \mathrm{pH}$ 6.8). HMM was prepared from fresh myosin by adding $\alpha$-chymotrypsin (59.3 units/mg protein, Sigma C4129) in $0.001 \mathrm{~N} \mathrm{HCl}$ to a final concentration of $0.04 \mathrm{mg} / \mathrm{ml}$ and incubated at room temperature for 7 minutes. The reaction was quenched by adding PMSF (Sigma-Aldrich) in ethanol to a final concentration of $0.5 \mathrm{mM}$ and dialyzed overnight against $1 \mathrm{~L}$ actin buffer $(0.25 \mathrm{M} \mathrm{KCl}, 0.25 \mathrm{M}$ Imidazole, 1 mM EGTA, $4 \mathrm{mM} \mathrm{MgCl}_{2}$, pH 7.4) with $2 \mathrm{mM}$ DTT at $4{ }^{\circ} \mathrm{C}$ with one buffer change. The dialysis product was clarified at 200,000g for 30 minutes and the HMM supernatant was stored in liquid nitrogen in aliquots containing $50 \%$ glycerol.

\section{Tryptic proteolysis of HMM loop 2}

HMM was selectively digested at the actin-binding loop 2 (cleaved HMM) as described in Bobkov et al. (1996) with minor modifications. Briefly, HMM was dialyzed against $0.02 \mathrm{M}$ $\mathrm{KCl}, 0.02 \mathrm{M}$ Tris- $\mathrm{HCl}$ and $1 \mathrm{mM}$ DTT at pH 7.4 to remove glycerol. HMM was reconcentrated to $0.65-1.1 \mathrm{mg} / \mathrm{ml}$ using centrifugal filtration units (Millipore Corp., Bedford, MA) at $10,000 \mathrm{~g}$. To protect loop 1 from digestion, the ionic strength was increased to $0.5 \mathrm{M} \mathrm{KCl}$ and ATP was added to $6 \mathrm{mM}$ (Mocz et al., 1984). Trypsin (proteolytic activity 0.08 BTEE units/mg; Sigma-Aldrich) was added to HMM to a final concentration of $0.05 \mathrm{mg} / \mathrm{ml}$ and incubated on ice for 6 minutes. Digestion was stopped using soybean trypsin inhibitor 
(Sigma-Aldrich) at a molar ratio of 3:1 to trypsin. Cleaved HMM was stored on ice in a $4^{\circ} \mathrm{C}$ refrigerator.

Digestion was assessed by electrophoresis on NuPAGE $12 \%$ Bis-Tris Gel in MOPS running buffer and gels were stained using SimplyBlue ${ }^{\mathrm{TM}}$ Safe Stain (Invitrogen, Carlsbad, CA). The molecular weight of fragments was determined by comparison to SeeBlue® Plus2 Protein Standard (Invitrogen) using the AlphaEaseFC (Alpha Innotech Corp., San Leandro, CA) molecular weight tool. As in previous experiments digestion of HMM mainly resulted in two fragments, the N-terminal fragment of S1 and the combined C-terminal region of S1 fragment and S2 (Bálint et al., 1975; Bobkov et al., 1996). Other contaminating fragments were present in low amounts.

\section{LMM purification}

LMM was purified from rat skeletal hind leg muscle as in (Margossian and Lowey, 1982). LMM purification follows same procedure as HMM purification through post-chymotryptic digestion of myosin dialysis. The dialysis precipitate product was clarified at 200,000g for 30 minutes and precipitate was resuspended in $200 \mu \mathrm{l}$ high salt buffer $(0.6 \mathrm{M} \mathrm{KCl}, 50 \mathrm{mM}$ $\mathrm{K}_{2} \mathrm{HPO}_{4}$, pH 7.0). Pellet was extracted with addition of $600 \mu \mathrm{l}$ ice cold $95 \%$ ethanol by stirring on ice for 2 hours. Collect precipitate by centrifugation at 20,000g for 30 minutes at $4^{\circ} \mathrm{C}$. Resuspend precipitate in $500 \mu \mathrm{l}$ high salt buffer and dialyze against $500 \mu \mathrm{l}$ high salt buffer over night at $4^{\circ} \mathrm{C}$ with one buffer change. Centrifuge at 200,000g for 30 minutes at $4^{\circ} \mathrm{C}$. Dialyze supernatant against $500 \mathrm{ml}$ actin buffer with $1 \mathrm{mM}$ DTT over night at $4^{\circ} \mathrm{C}$ with one buffer change. Collect precipitated LMM by centrifugation at 20,000g for 30 minutes at $4^{\circ} \mathrm{C}$. Resuspend pellet in small volume ( $\left.30 \mu \mathrm{l}\right)$ myosin buffer $(300 \mathrm{mM} \mathrm{KCl}, 25 \mathrm{mM}$ Imidazole, $1 \mathrm{mM}$ EGTA, $4 \mathrm{mM} \mathrm{MgCl} 2,1 \mathrm{mM}$ DTT, $\mathrm{pH}$ 7.4). Store in $50 \%$ glycerol at $-20^{\circ} \mathrm{C}$.

\section{Actin purification and polymerization}

F-actin was prepared from rat skeletal muscle as described in Pardee and Spudich (1982). First, acetone powder was purified from fresh rat hind leg skeletal muscle. Fresh tissue was homogenized in a food processor in $\mathrm{KPi} / \mathrm{KCl}$ solution $\left(0.15 \mathrm{M} \mathrm{KH}_{2} \mathrm{PO}_{4}, 0.10 \mathrm{M} \mathrm{KCl}, 1 \mathrm{mM}\right.$ $\mathrm{NaN}_{3}, \mathrm{pH} 6.5$ ). The homogenate was stirred for 10 minutes on ice, then clarified at $1700 \mathrm{~g}$ 
for 15 minutes. Pellet was extracted in $0.05 \mathrm{M} \mathrm{NaHCO}_{3}$ on ice for 10 minutes and clarified as before. This clarification was repeated 2 times. Pellet was extracted in 1 mM EDTA, pH 7.0 for 5 minutes on ice and clarified as before. EDTA clarification was repeated two times. Pellet was extracted with acetone for 10 minutes at room temperature and clarified for 5 minutes at $1700 \mathrm{~g}$, extraction and clarification were repeated 4 times. Pellet was extracted with acetone for 10 minutes, then filtered by vacuum on filter paper. Acetone powder dried over night at room temperature and was stored at $-20^{\circ} \mathrm{C}$.

Actin was purified from acetone powder by stirring on ice in actin extraction buffer $(2 \mathrm{mM}$ Tris Base, $0.2 \mathrm{mM}$ ATP, $0.2 \mathrm{mM} \mathrm{CaCl}_{2}, 0.005 \% \mathrm{NaN}_{3}, 1 \mathrm{mM}$ DTT, pH 8.0) for 30 minutes and actin was collected by centrifugation at $10,000 \mathrm{~g}$ for 20 minutes. Remaining actin was extracted from pellet by stirring in actin extraction buffer for 10 minutes on ice and collected by centrifugation as before. Supernatant from both actin collections were combined and polymerized over night by raising the $\mathrm{KCl}$ concentration to $100 \mathrm{mM}$, the $\mathrm{MgCl}_{2}$ concentration to $2 \mathrm{mM}$, and the ATP concentration to $1 \mathrm{mM}$. The next day, tropomyosin was removed in a high salt wash by slowly increasing the KCL concentration to $500 \mathrm{mM}$ while stirring. F-actin was sedimented by centrifugation at $200,000 \mathrm{~g}$ for 1 hour. Pelleted actin was depolymerized by resuspension in $1.5 \mathrm{ml}$ actin extraction buffer per gram of starting acetone powder for 1 hour, undisturbed, after which the pellets were homogenized. Homogenate was further depolymerized by dialysis at $4^{\circ} \mathrm{C}$ against $1 \mathrm{~L}$ actin extraction buffer for 3 days with a fresh buffer change each day using 3500-5000 MWCO dialysis membrane. G-actin was clarified by centrifugation at 200,000g for 1 hour. DTT concentration was raised to $10 \mathrm{mM}$ in G-actin supernatant. G-actin was stored in liquid nitrogen in aliquots containing $50 \%$ glycerol.

F-actin was polymerized from G-actin aliquots by combining G-actin with polymerization buffer ( $1 \mathrm{M} \mathrm{KCl}, 20 \mathrm{mM} \mathrm{MgCl}_{2}, 10 \mathrm{mM} \mathrm{ATP}$ ) at a 1:10 ratio by volume and allowed to polymerize over night at $4^{\circ} \mathrm{C}$ or in 1 hour at room temperature. 


\section{Actin biotinylation}

F-actin was biotinylated as in Rao, et al. (2009) by pelleting the actin at 200,000g for 30 minutes, and the pellet resuspended in buffer containing $0.1 \mathrm{M}$ HEPES, $2 \mathrm{mM} \mathrm{MgCl} 2,1 \mathrm{mM}$ ATP, 1 mM DTT, pH 8.5. NHS-Biotin was added to the resuspended filament solution to a final concentration of $0.83 \mathrm{mM}$ and allowed to incubate at room temperature for 2 hours. The actin-biotin solution was centrifuged at 200,000g for 30 minutes. The biotinylated actin pellet was washed in actin polymerization buffer $(100 \mathrm{mM} \mathrm{KCl}, 25 \mathrm{mM}$ Imidazole, 4 $\mathrm{mM} \mathrm{MgCl}, 1 \mathrm{mM}$ ATP, $1 \mathrm{mM}$ DTT, $\mathrm{pH}$ 7.4) and centrifuged at 200,000g for 15 minutes. The wash was repeated 2 more times. The actin pellet was resuspended in polymerization buffer containing tetramethylrhodamine isothiocyanate (TRITC) phalloidin to label for 24 hours before use.

\section{Biotinylated actin microspheres}

Biotinylated F-actin was coupled to streptavidin-coated polystyrene microspheres ( 0.97 or $1.04 \mu \mathrm{m}$ diameter, Bangs Laboratories, Inc., Fishers, IN) by combining a suspension containing $\sim 4 \times 10^{8}$ microspheres with a $3 \mu \mathrm{M}$ final concentration of biotinylated actin filaments and allowed to couple with overnight mixing at $4^{\circ} \mathrm{C}$. Excess biotinylated actin was removed by pelleting the microspheres at 10,000g. Microspheres were blocked by incubating in $1 \mathrm{mg} / \mathrm{ml}$ BSA in actin buffer for 15 minutes and washed in actin buffer three times. Biotinylated actin-coated microspheres were stored in actin buffer with $10 \mathrm{mM}$ DTT at $4^{\circ} \mathrm{C}$ for up to 2 weeks. Actin-coated microspheres were diluted in actin buffer immediately before use.

\section{cMyBP-C full-length and N-terminal fragment preparation}

Full-length and cMyBP-C N-terminal fragments were provided by the Warshaw Lab (University of Vermont). These were bacterially expressed from mouse cardiac cDNA using a pET expression system (Novagen, Madison, WI) (Sadayappan et al., 2005) Two different $\mathrm{N}$-terminal fragments were expressed, wild type C0C3 (amino acids 1-539) and C0C3 with aspartic acids (D) to mimic phosphorylation at S273, S282, S302, and S307 (referred to as C0C3-4D). Fragments used in HMM bond lifetime experiments were expressed containing a 
biotin attached to the N-terminus of domain $\mathrm{C} 0$ by a 15 amino acid peptide tag (Avidity, LLC, Aurora, CO).

\section{Biotinylated COC3 and COC3-4D microspheres}

Biotin-tagged C0C3 and C0C3-4D were coupled to $1.04 \mu \mathrm{M}$ diameter streptavidin-coated microspheres by suspending a solution of microspheres containing $\sim 3.2 \times 10^{8}$ microspheres with a saturating concentration of either the $\mathrm{C} 0 \mathrm{C} 3$ or C0C3-4D fragment and allowed to mix overnight at $4^{\circ} \mathrm{C}$. Fragment-coated microspheres were blocked with $1 \mathrm{mg} / \mathrm{ml} \mathrm{BSA}$ to reduce non-specific interactions. C0C3 microspheres and C0C3-4D microspheres were stored in actin buffer with $10 \mathrm{mM}$ DTT on ice at $4^{\circ} \mathrm{C}$ for up to 3 days. Microspheres were diluted immediately before use in actin buffer containing $10 \mathrm{mM}$ DTT. An ultrasonic dismembrator was pulsed in diluted microsphere solution immediately before use to break apart clumps of microspheres.

\section{A1.2 Actin-HMM Force Spectroscopy and In Vitro Motility}

\section{Laser trap setup and calibration}

The laser trap used in these experiments was the same as described in Guilford et al. with minor modifications (Guilford et al., 2004). The laser has been replaced by a $25 \mathrm{~W}, 1020$ nm fiber laser (SPI Lasers, Santa Clara, CA), the digital signal processor card has been replaced by a field-programmable gate array (National Instruments, Austin, TX), and a user interface was created in LabVIEW (National Instruments). Back focal plane interferometry was used to measure the position of a trapped microspheres relative to the trap center, from which measurements of displacement and force were calculated. The sensitivity of the interferometer and the trap stiffness were calibrated by the step response method (Dupuis et al., 1997; Svoboda and Block, 1994) and by fits to the power spectral density (Allersma et al., 1998).

\section{Flow cell assembly}

Flow cells used in laser trap experiments were prepared as in Guo and Guilford (2006). Glass bead targets (3-10 $\mu \mathrm{m}$ diameter) were aerosolized in reagent alcohol and applied to 
glass coverslips. Bead-coated coverslips were then coated with nitrocellulose and assembled into flow cells using optical adhesive (Norland Products, Inc. Cranbury, NJ) to adhere a nitrocellulose- and target-coated coverslip to a second coverslip, separated by mylar shims. The total volume of each flow cell was approximately $30 \mu \mathrm{l}$.

\section{Flow cell loading for actin-HMM force spectroscopy experiments}

HMM was incubated in the flow cell for 1 minute. The flow cell was then blocked with 1 $\mathrm{mg} / \mathrm{ml} \mathrm{BSA}$ in actin buffer for 1 minute and washed with actin buffer. A suspension of actin buffer and actin-coated microspheres was added to the flow cell. An oxygen scavenger system $(0.125 \mathrm{mg} / \mathrm{ml}$ glucose oxidase, $0.0225 \mathrm{mg} / \mathrm{ml}$ catalase, $2.87 \mathrm{mg} / \mathrm{ml}$ glucose $)$ and $0.01 \mathrm{mM}$ DTT were included in the actin buffer and the microsphere suspension. The flow cell was placed onto a piezoelectric microscope stage (nPoint Inc., Middleton, WI).

\section{Actin-HMM bond lifetimes}

Flow cells were loaded for actomyosin bond lifetime experiments as follows. HMM was diluted to $10 \mu \mathrm{g} / \mathrm{ml}$ in actin buffer was incubated in the flow cell for 1 minute. The flow cell was then blocked with $1 \mathrm{mg} / \mathrm{ml} \mathrm{BSA}$ in actin buffer for 1 minute and washed with actin buffer. A suspension of actin buffer and actin-coated microsphere was added to the flow cell. An oxygen scavenger system $(0.125 \mathrm{mg} / \mathrm{ml}$ glucose oxidase, $0.0225 \mathrm{mg} / \mathrm{ml}$ catalase, $2.87 \mathrm{mg} / \mathrm{ml}$ glucose) and $0.01 \mathrm{mM}$ DTT were included in the actin buffer and the microsphere suspension. The flow cell was placed onto a piezoelectric microscope stage (nPoint Inc., Middleton, WI).

All laser trap voltage calibration and data measurements were performed using Athyrio, a program developed by Will Guilford. The stiffness of the laser and the sensitivity of the quadrant photodiode detector (QD) were calibrated using the step response method as detailed in Dupuis, et al. (1997). An actin-coated microsphere was captured in a laser trap and the laser was stepped a distance equal to that used in bond rupture experiments (200 $\mathrm{nm})$. The time for the microsphere to return to the center of the laser trap was recorded for steps 1 second in duration in alternating directions. The measured time response was related to the trap stiffness using the following equation: 


$$
\ln \left(\frac{x_{0}}{x(t)}\right)=\frac{\alpha_{t r a p}}{\beta} t
$$

where $x_{0}$ is the distance the laser was stepped, $x(t)$ is the distance of the actin-coated microsphere from the post-step trap position at time $t, \alpha_{\text {trap }}$ is the stiffness of the laser trap, and $\beta$ is the Stokes' drag coefficient of a microsphere $(\beta=6 \pi \eta r$, where $\eta$ is viscosity and $r$ is microsphere radius). $\alpha_{\text {trap }}$ was calculated from the slope of the linear regression line of a plot of the log term against time and $\beta$. The sensitivity of the QD was calibrated by recording the voltage output of the QD in response to stepping the laser a known distance within the linear range of the detector.

Actomyosin bond lifetime experiments were performed by trapping an actin-coated microsphere in the laser and stepping it $200 \mathrm{~nm}$ toward the HMM-coated target. The actincoated microspheres were brought into contact for 1 second with a target coated with 10 $\mu \mathrm{g} / \mathrm{ml} \mathrm{HMM}$. The laser trap was stepped $200 \mathrm{~nm}$ away from the target and held there for 1 second before repeating this cycle. The load applied to the actomyosin bond was calculated from the difference in voltage from the time the laser was stepped away from the HMMcoated target and the time the actin-coated microsphere returned to the center of the laser trap using stiffness of the laser and the sensitivity of the detector. The initial separation between the trapped microsphere and the target was random, resulting in random compressive and tensile loads applied to the actomyosin bond. Over a large number of experiments this resulted in a range of step loads being applied to actin-HMM bonds using the laser trap. All experiments were performed in the absence of nucleotide. Bond formation was determined by the trapped microsphere remaining in contact with the target after the laser trap was stepped away from it. Bond rupture was determined by the microsphere returning to its original position away from the target. Bond lifetime was defined as the time elapsed between stepping the laser away from the HMM-coated target and bond rupture. Bonds shorter than $4 \mathrm{~ms}$ and those indicative of more than one HMM head bound were excluded from analysis. 
Actomyosin bonds were binned by load into $2 \mathrm{pN}$ bins and plotted against bond lifetimes. We fit the data with a two-pathway model of dissociation with weight $t_{c s}(f)^{2}$ as described in Pereverzev et al. (2005):

$$
\frac{1}{t_{c s}(f)}=k_{c}^{0} \exp \left(\frac{x_{c} f}{k_{B} T}\right)+k_{s}^{0} \exp \left(\frac{x_{s} f}{k_{B} T}\right)
$$

where $t_{c s}(f)$ is the mean bond lifetime at force $f$ for a catch bond, $k_{c}{ }^{o}$ and $k_{s}{ }^{o}$ are the dissociation constants for unbinding through the catch and slip pathways, respectively, $x_{c}$ and $x_{s}$ are the characteristic bond lengths of the catch and slip pathways, respectively, and $k_{B} T$ is thermal energy. $\mathrm{x}_{c}<0$ for the catch pathway to be a dissociation barrier. The bond lifetime reaches a maximum at the critical force:

$$
f_{\text {crit }}=\frac{k_{B} T}{x_{s}-x_{c}} \ln \left(-\frac{k_{c}^{0} x_{c}}{k_{s}^{0} x_{s}}\right)
$$

where

$$
-\frac{k_{c}^{0} x_{c}}{k_{s}^{0} x_{s}}>1
$$

\section{Actin-HMM fraction of bonds surviving}

Thomas, et al. proposed the allosteric catch bond model in which a ligand-bound protein may exist in two conformations with the transition between them being a function of force applied to the bond (Thomas et al., 2008). One conformation possesses a greater unbinding rate and a fast-binding state, and the second possesses a lower unbinding rate and a slowbinding pathway. Rao, et al. suggest that actomyosin catch bond results from allostery (Rao et al., 2011).

The fraction of bonds surviving over time was calculated using the equation: 


$$
B(t)=\frac{b(t)}{b^{0}}
$$

where $B(t)$ is the fraction of bonds surviving at time $t, b^{o}$ is the initial number of bonds and $b(t)$ is the number of bonds remaining at time $t$. Fraction surviving was plotted against time. The data were fit by a double exponential decay:

$$
B(t)=B_{1}^{0} \exp \left(-\lambda_{1} t\right)+\left(1-B_{1}^{0}\right) \exp \left(-\lambda_{2} t\right)
$$

where $B(t)$ is fraction of bonds surviving at time $t, B_{1}{ }^{0}$ is the initial fraction surviving in the fast-binding pathway, and $\lambda_{i}$ is the rate of dissociation from the fast- or slow-binding state ( 1 and 2, respectively). Independent variables of the regression were weighted by the value of their dependent variable (the lifetime of the bond).

\section{Actin-HMM specific 2D on-rate}

The load-dependent 2D on-rate (the rate of bond formation) was measured over a range of compressive forces using a method reported previously by us (Rao et al., 2011) and others (Chen et al., 2008). Flow cells were loaded as described for actin-HMM bond lifetime experiments with intact or cleaved HMM applied at a concentration of $80 \mu \mathrm{g} / \mathrm{ml}$. The surface density was increased because on-rate experiments require a surface density high enough to overcome the limitations of diffusion in the ligand-receptor interaction, whereas bond lifetime measurements necessitate low site densities to ensure single bond events (Snook and Guilford, 2012). An actin-coated microsphere was brought into contact with an HMM-coated target for $1 \mathrm{~s}$, then rapidly stepped $200 \mathrm{~nm}$ away perpendicular to the filament axis for $1 \mathrm{~s}$. This cycle was repeated 30 times.

Bond formation was observed directly in the laser trap. We observe a change in the mean signal magnitude and variance (due to Brownian motion) when the first bond forms between the trapped microsphere and the target. The time to bond formation $\left(t_{b}\right)$ is the elapsed time between arrival of the microsphere at the target and the moment of bond formation (Figure 4, position iii). 2D on-rate is inversely related to $t_{b}$ by Equation 3, where 
$m_{r}$ is the site density of HMM on the nitrocellulose-coated targets (3300 heads $/ \mu \mathrm{m}^{2}$ ) (Guo and Guilford, 2004), $m_{l}$ is the density of available myosin binding sites on actin-coated microspheres ( 637 sites $/ \mu \mathrm{m}^{2}$ ), and $A_{c}$ is the effective contact area between the target and trapped microsphere $\left(0.14 \mu \mathrm{m}^{2}\right)$ as calculated using equations $1 \& 2$ in Rinko et al. (2004). Specific 2D on-rate data were fit using a "reverse Bell model" as in Snook and Guilford (2010):

$$
k_{o n}(f)=k_{o n}^{0} \exp \left(f \cdot x_{\gamma a} / k_{B} T\right)
$$

where $k_{o n}(f)$ is the load-dependent on-rate, $k_{o n}{ }^{0}$ is the unloaded on-rate, $f$ the applied load, $x_{\gamma a}$ the characteristic bond length of association, $k_{B}$ the Boltzmann constant, and $T$ the absolute temperature. The perpendicular, compressive force $\left(f_{c}\right)$ between the microsphere and the target during bond formation was calculated as the difference between the distance the laser was stepped and the distance the microsphere actually moved after bond rupture, multiplied by the stiffness of the laser trap. Negative $f_{c}$ values indicate no contact between the microsphere and the target, resulting in a time-averaged tensile rather than compressive load.

\section{Digested HMM in vitro motility assay}

Motility was measured as described by others (Guo and Guilford, 2004; Kron et al., 1991; Uyeda et al., 1991; Warshaw et al., 1990) at $30^{\circ} \mathrm{C}$. Briefly, intact, cleaved, or fully digested (no trypsin inhibitor) HMM was applied to a flow cell made of a glass slide and nitrocellulose-coated coverslip separated by mylar shims. After a 1 minute incubation, the flow cell was blocked with 1\% BSA (Sigma) in actin buffer with 10 mM DTT for 1 minute, followed by addition of TRITC-phalloidin labeled actin filaments. After a 1 minute incubation, the flow cell was washed with actin buffer with $10 \mathrm{mM}$ DTT and motility buffer (25 mM KCl, 25 mM Imidazole, 1 mM EGTA, 4 mM MgCl 2,10 mM DTT, 1 mM ATP, 0.5\% Methylcellulose, $\mathrm{pH}$ 7.4) containing an oxygen scavenger system was applied. The flow cell was incubated for 1 minute at $30^{\circ} \mathrm{C}$ before imaging. The mean velocities of filaments and 
the fraction of filaments that move were determined using the tracking algorithm described in Snook, et al. (2008).

\section{A1.3 cMyBP-C Phosphorylation-Dependent Crossbridge Binding}

\section{COC3 force spectroscopy}

For actin-COC3/4D bond lifetime measurements: $1 \mathrm{nM}$ C0C3 or C0C3-4D was incubated in the flow cell for 2 minutes. The flow cell was then blocked with $1 \mathrm{mg} / \mathrm{ml}$ BSA in actin buffer for 2 minutes and washed with actin buffer. A suspension of actin buffer and biotinylated actin microspheres was added to the flow cell. An oxygen scavenger system and $0.01 \mathrm{mM}$ DTT were included in the actin buffer and the bead suspension. Bond lifetime measurements were made between C0C3 or C0C3-4D on the flow cell surface targets and an actin-coated microsphere captured in the laser trap.

For HMM-COC3/4D bond lifetime measurements: $10 \mu \mathrm{g} / \mathrm{ml}$ HMM was incubated in the flow cell for 2 minutes. The flow cell was then blocked with $1 \mathrm{mg} / \mathrm{ml}$ BSA in actin buffer for 10 minutes and washed with actin buffer. A suspension of actin buffer and C0C3- or C0C3-4Dcoated microspheres was added to the flow cell. An oxygen scavenger system and $0.01 \mathrm{mM}$ DTT were included in the actin buffer and the microsphere suspension. All HMM experiments were performed in the absence of nucleotide. Bond lifetime measurements were made between HMM on the flow cell surface targets and a C0C3- or C0C3-4D-coated microsphere captured in the laser trap.

\section{COC3 bond lifetimes}

Protein-coated microspheres were captured in a laser trap and brought into contact with a protein-coated target for 1 second. The laser trap was stepped $200 \mathrm{~nm}$ away from the target and held there for 1 second before repeating this cycle. The initial separation between the trapped microsphere and the target was random, resulting in random tensile loads experienced by the bond between actin or HMM and a cMyBP-C fragment. Over a large number of experiments this resulted in a range of random step loads being applied to actin or HMM-C0C3/4D bonds using the laser trap. Bond formation was accompanied by a 
decrease in Brownian motion and a small shift in displacement, as well as the trapped microsphere remaining in contact with the target after the laser trap was stepped away from it. Bond rupture was accompanied by return of the microsphere to its original position away from the target. Bond lifetime was defined as the time elapsed between stepping the laser away from the HMM-coated target and bond rupture. The tensile load experienced by a bond was calculated from the stiffness of the laser trap and the distance a microsphere moved to return to the center of the laser trap.

Bond lifetimes were divided into $2 \mathrm{pN}$ bins, within any one of which the lifetimes were exponentially distributed. $95 \%$ confidence intervals of the mean of the exponentially distributed bond lifetime data were calculated in each $2 \mathrm{pN}$ force bin using Equation 1.

Control experiments were performed with blank targets (no C0C3- or C0C3-4D on targets with actin-coated microspheres, and no HMM on targets with C0C3- or C0C3-4D-coated microspheres).

\section{COC3 zero load force spectroscopy}

Bond lifetimes between actin or HMM and C0C3 or C0C3-4D at near-zero load were measured as in (Guo and Guilford, 2006). Briefly, and actin-coated microsphere was held adjacent to a C0C3/-4D-coated target or a C0C3/-4D-coated microsphere was held adjacent to an HMM-coated target and bonds were allowed to form and break under low, semirandom loads. Bond formation was identified by a decrease in Brownian motion accompanied by a small shift in baseline, and bond rupture by a reversal of those changes. Fits to power spectral density were used to calibrate the laser trap stiffness and detector sensitivity.

\section{Actin-COC3 bond formation measurements}

The frequency of bond formation was measured over a range of C0C3 surface densities to determine a concentration at which $50 \%$ or greater binding occurs. To measure the frequency of bond formation, an actin-coated microsphere was stepped in and out of contact with a C0C3-coated target surface with a range surface densities (50-400 nM COC3). 
The frequency of bond formation was calculated as the number of trapped-microsphere target contacts resulting in a bond divided by the total number of contacts. Based on the results from these measurements, all bond formation experiments were performed while incubating $400 \mathrm{nM} \mathrm{C0C3}$ or C0C3-4D in the flow cell.

The probability of bond formation was measured as a function of contact time between the actin-coated microsphere and the cMyBP-C fragment-coated target. Flow cells were assembled as previously described with C0C3 or C0C3-4D adsorbed to the nitrocellulosecoated target surface and biotinylated actin coupled to the laser-trapped microsphere. Similar to Chesla et al. (1998), we determined adhesion probability as a function of contact time between two surfaces by repeatedly stepping an actin-coated microsphere into contact with a stationary cMyBP-C fragment-coated target and counting the number of bonds formed for a fixed number of contacts. The molecules were stepped in and out of contact with a constant step-out time of 1000 ms and a range of contact (step-in) times (5$3200 \mathrm{~ms}$ ). The adhesion probability, the number of times a bond was observed divided by the total number of contacts, was plotted as a function of contact time. Adhesion probability $\left(P_{a}\right)$ data were fit according to Chesla et al. (1998) using Equation 7. Because $m_{r}$ and $m_{l}$ are as yet unknown to us, we fit our data by lumping unknown constants $A_{c}, m_{r}$, and $m_{l}$ with $k_{f}^{0}$ into one fitted parameter.

Bond formation in the laser trap must be measured at a higher surface density of the target protein (COC3 or C0C3-4D in these experiments) in order to overcome the limitations of diffusion in the ligand-receptor interaction, whereas bond lifetime measurements necessitate low site densities to ensure single bond events (Snook and Guilford, 2012). The frequency of bond formation was measured over a range of COC3 surface densities to find a concentration at which $50 \%$ or greater binding occurs. Based on the results from these measurements, all bond formation experiments were performed by applying $400 \mathrm{nM}$ COC 3 or C0C3-4D to the surface of the flow cell. 
Time to bond formation $\left(t_{b}\right)$ was also measured to validate our adhesion probability measurements. $t_{b}$ was measured between single C0C3 or C0C3-4D binding sites on actincoated microspheres using the method described in Appendix 1.

cMyBP-C N-terminal fragment in vitro motility assay Actomyosin motility in the presence of the COC3 cMyBP-C N-terminal fragments was measured as described by others (Saber et al., 2008; Weith et al., 2012a) at $30^{\circ} \mathrm{C}$. Briefly, 32 $\mu \mathrm{g} / \mathrm{ml} \mathrm{HMM}$ was applied to a flow cell made of a glass slide and nitrocellulose-coated coverslip separated by mylar shims. After a 1 minute incubation, the flow cell was blocked with $1 \%$ BSA in actin buffer with $10 \mathrm{mM}$ DTT for 1 minute, followed by addition of TRITCphalloidin labeled actin filaments. After a 1 minute incubation, the flow cell was washed with actin buffer with $10 \mathrm{mM}$ DTT and exchanged with a final motility buffer ( $25 \mathrm{mM} \mathrm{KCL}$, 25 mM Imidazole, 1 mM EGTA, 4 mM MgCl 2,10 mM DTT, 1 mM ATP, 0.5\% Methyl Cellulose, $\mathrm{pH}$ 7.4) containing an oxygen scavenger system and COC3. The flow cell was incubated for 1 minute at $30^{\circ} \mathrm{C}$ before imaging. The mean velocities of filaments and the fraction of filaments that move were determined using the tracking algorithm described in Snook, et al. (2008).

\section{A1.4 cMyBP-C Nitrosylation}

\section{Nitrosylation donors}

A $10 \mathrm{mM}$ stock solution of S-nitroso-L-cysteine (SNO-L-cys) was prepared from L-cysteine and stored at $-80^{\circ} \mathrm{C}$ for up to 1 month. A $10 \mathrm{mM}$ stock solution of L-nitrosoglutathione (GSNO) was prepared from L-glutathione and stored at $-80^{\circ} \mathrm{C}$ for up to 1 month.

Diethylamine-NONOate (DEA-NONOate; Cayman Chemical Company, Ann Arbor, MI) was obtained commercially, diluted to $2 \mathrm{mg} / \mathrm{ml}$ in DMSO and stored at $-20^{\circ} \mathrm{C}$. Stock donors were diluted to working concentrations in actin buffer immediately before use.

\section{Coumarin switch assay}

S-nitrosylation was measured using a modification (Han et al., 2008) of Jaffrey's biotin switch assay (Jaffrey and Snyder, 2001). AMCA-HPDP was used rather than biotin-HPDP 
during labeling to allow S-nitrosylated proteins to be imaged in-gel prior to staining total protein; this aids in normalization of fluorescence against protein mass in each band. Briefly, a NO donor was used to nitrosylate all solvent-accessible, nitrosylatable cysteines. Proteins were then denatured and treated with a blocking agent (methanethiosulfonate, MMTS) to alkylate reduced cysteines. Sodium ascorbate was used to break S-NO bonds, thereby newly reducing cysteines that were originally nitrosylated by the donor treatment. Newly reduced cysteines were labeled with a thiol-reactive coumarin (AMCA-HPDP) and visualized under ultraviolet light in SDS-PAGE gels.

Three samples were used for each switch experiment. One donor-treated sample was processed as described above (test sample). A control sample was left untreated to determine the background signal when there was no nitrosylation. Finally, we omitted MMTS block from one sample in order to label all the cysteines residues in the protein (noblock sample).

Gels were imaged using a Bio-Rad ChemiDoc MP ${ }^{\mathrm{TM}}$ Imaging System (Bio-Rad Laboratories Inc., Hercules, CA). Fluorescence signals were collected at $530 \mathrm{~nm}(530 / 28 \mathrm{BP})$ to measure AMCA labeling. After AMCA imaging, gels were stained with colloidal coomassie and imaged in brightfield for total protein. S-nitrosylation and total protein were determined through optical intensity measurements of the protein bands following SDS-PAGE using Image Lab 4.0.1 software (Bio-Rad Laboratories, Inc.).

Protein nitrosylation was quantified by collecting the AMCA fluorescence signal and subsequently stained with colloidal coomassie to determine the mass for each protein band. Fluorescence was normalized against total protein. The combination of normalized fluorescence values from our three samples allowed us to estimate the number of nitrosylated cysteines in the test sample.

The ratio of AMCA and total protein band volumes ( $\left.\mathrm{R}_{\text {sample }}\right)$ was obtained for each sample: 


$$
\mathrm{R}_{\text {sample }}=\frac{\mathrm{AMCA}_{\text {sample }}}{\text { Total Protein }}
$$

Donor-treated samples were adjusted for background and autofluorescence by subtracting the ratio of the control sample from each. The adjusted ratio of the labeled sample (cysteines able to be nitrosylated) was divided by the no-block sample (all cysteines in protein) to give the fraction of cysteines in the protein able to be nitrosylated $\left(\mathrm{C}_{\text {label }}\right)$ :

$$
C_{\text {label }}=\frac{R_{\text {label }}-R_{\text {control }}}{R_{\text {no block }}-R_{\text {control }}}
$$

Quantification of cysteines able to be nitrosylated ( $\left.\mathrm{N}_{\text {label }}\right)$ was performed by multiplying $\mathrm{C}_{\text {label }}$ by the total number of cysteines present in the protein (n) reported in the nucleotide sequence for the protein.

$$
\mathrm{N}_{\text {label }}=\mathrm{n} \times \mathrm{C}_{\text {label }}
$$

There are 22 cysteine residues present in mouse cMyBP-C and 7 present in the C0C3 fragment of the protein (UniProt accession No. 070468).

\section{Nitrosylation Treatment for cMyBP-C Motility}

Exposure to NO donors takes place in reducing agent-free solutions.

cMyBP-C was nitrosylated using SNO-L-cysteine as a NO donor at a final concentration of $400 \mu \mathrm{M}$. Donor was added to protein and allowed to incubate on ice in the dark for 5 minutes. Donor-treated samples were added to motility buffer as described below prior to degassing. Solutions were placed under vacuum for 20 minutes to denature remaining unreacted donor. A control sample of denatured donor was prepared by diluting donor to $400 \mu \mathrm{M}$ in a volume of actin buffer equal to that of cMyBP-C used in the experimental sample, and was placed under vacuum for 20 minutes to denature unreacted donor. 
cMyBP-C nitrosylation and in vitro motility assay

cMyBP-C was dialyzed against $1 \mathrm{~L}$ actin buffer over night to remove DTT before use in nitrosylation experiments. A DTT-free actomyosin motility was performed as described elsewhere (Snook et al., 2008) with modifications to include cMyBP-C. Motility was measured at $30^{\circ} \mathrm{C}$ with treated and control cMyBP-C, as well as a cMyBP-C-free control with and without decomposed donor. All reducing agent-free buffers were degassed under vacuum on ice for 20 minutes in small aliquots to remove oxygen and were re-degassed after 4 flow cells or 20 minutes, whichever came first.

HMM was applied to a flow cell at a final concentration of $100 \mu \mathrm{g} / \mathrm{ml}$ in actin buffer containing $10 \mathrm{mM}$ DTT. After a 1 minute incubation, the flow cell was blocked with 1 $\mathrm{mg} / \mathrm{ml} \mathrm{BSA}$ in actin buffer with $10 \mathrm{mM}$ DTT for 1 minute. TRITC-phalloidin labeled actin filaments were introduced, followed by a wash with DTT-free degassed actin buffer. One volume of motility buffer was added containing treated or control cMyBP-C, or a decomposed donor control. Actin filament movement was recorded as described above.

\section{Nitrosylation treatment for cMyBP-C binding to LMM}

Exposure to NO donors takes place in reducing agent-free solutions.

cMyBP-C was nitrosylated using SNO-L-cysteine as a NO donor at a final concentration of $400 \mu \mathrm{M}$. Donor was added to protein and allowed to incubate on ice in the dark for 5

minutes. The solution was then placed under vacuum for 20 minutes to denature remaining unreacted donor. Two control samples of filamentous LMM were also prepared in the absence of cMyBP-C - one in which LMM was allowed to react with the donor and one in which decomposed donor was added. Nitrosylation was performed before cMyBP-C was combined with LMM filaments.

\section{LMM filament assembly and cMyBP-C binding}

To measure cMyBP-C binding to LMM, a centrifugation (spin-down) and C-protein binding assay was performed as in Okagaki et al. (2007). Briefly, $1 \mathrm{~L}$ filament assembly buffer $(100$ $\mathrm{mM} \mathrm{KCl}, 25 \mathrm{mM}$ Imidazole, $1 \mathrm{mM}$ EGTA, $4 \mathrm{mM} \mathrm{MgCl}$, $\mathrm{pH}$ 7.4) was vacuumed for a minimum 
of one hour to remove air. LMM was diluted to $3 \mu \mathrm{M}$ in myosin buffer ( $300 \mathrm{mM} \mathrm{KCL}, 25 \mathrm{mM}$ imidazole, $1 \mathrm{mM} \mathrm{EGTA}, 4 \mathrm{mM} \mathrm{MgCl}$, $\mathrm{pH}$ 7.4) immediately before dialysis. LMM was polymerized by dialysis against filament assembly buffer for 1 hour. LMM filaments were incubated with cMyBP-C or nitrosylated cMyBP-C on ice for 30 minutes at a final concentration of $0.25 \mu \mathrm{M}$, a concentration at which $50 \%$ binding saturation occurs (Okagaki et al., 2007). Samples of assembled LMM filaments and unbound cMyBP-C remained as controls. Samples were centrifuged at 100,00 g for 40 minutes at $4^{\circ} \mathrm{C}$. Supernatant was collected and stored on ice while pellets were resuspended in a volume of filament assembly buffer equal to the pre-centrifugation volume of each sample. All supernatant and pellet samples were diluted in sample buffer, heated, and gel electrophoresis was performed using 12\% Bis-Tris gels in MOPS running buffer.

Relative LMM assembly and cMyBP-C binding was measured by comparing gel band volumes using Image Lab 4.0.1 software (Bio-Rad Laboratories). The volume of each LMM and cMyBP-C band was measured. Volume measurements for all LMM bands were adjusted by the volume of LMM in the supernatant of the control sample. Volume measurements for all cMyBP-C bands were adjusted by the volume of $\mathrm{cMyBP}-\mathrm{C}$ in the pellet of the control sample. The pelleted fraction is

$$
\mathrm{P}=\frac{\mathrm{V}_{\text {pellet }}}{\mathrm{V}_{\text {pelect }}+\mathrm{V}_{\text {supermatant }}}
$$

where $V_{\text {pellet }}$ is the volume of the pellet band and $V_{\text {supernatant }}$ is the volume of the supernatant band for the same sample. $P$ was calculated for LMM and cMyBP-C in each sample. 


\section{Appendix 2: Fitted Parameters}


Table 3: The fitted parameters of the two-pathway dissociation model for actin-HMM bond lifetime vs load data (Figure 6a and b, Page 23). HMM loop 2 is intact.

\begin{tabular}{lcccc}
\hline \multicolumn{3}{c}{ Loop 2 Intact } \\
\hline \multirow{2}{*}{ Ionic Strength $(\mathrm{mM})$} & \multicolumn{2}{c}{ Catch Pathway } & \multicolumn{2}{c}{ Slip Pathway } \\
\cline { 2 - 5 } & $\mathrm{k}_{\mathrm{c}}^{0}, \mathrm{~s}^{-1}$ & $\mathrm{x}_{\mathrm{c}}, \mathrm{nm}$ & $\mathrm{k}_{\mathrm{s}}{ }^{0}, \mathrm{~s}^{-1}$ & $\mathrm{x}_{\mathrm{s}}, \mathrm{nm}$ \\
\hline 25 & 310 & -7.2 & 7.1 & 0.2 \\
\hline 145 & 1950 & -10.5 & 5.8 & 0.2 \\
\hline
\end{tabular}

Errors have not been included because fits were based on a weighted regression, which invalidates the error estimates. Data were fit using Equation 5.

Table 4: The fitted parameters of the single bound pathway dissociation model for actin-myosin bond lifetime vs load data (Figure 6a and b, Page 23). HMM loop 2 is cleaved.

\begin{tabular}{lcc}
\hline \multicolumn{3}{c}{ Loop 2 Cleaved } \\
\hline Ionic Strength $(\mathrm{mM})$ & $\mathrm{k}^{0}, \mathrm{~s}^{-1}$ & $\mathrm{x}, \mathrm{nm}$ \\
\hline 25 & 11.1 & -0.08 \\
\hline 145 & 4.1 & 0.28
\end{tabular}

Errors have not been included because fits were based on a weighted regression, which invalidates the error estimates. Data were fit using Equation 6. 
Table 5: The fitted parameters of a double exponential decay for the fraction of actin-HMM bonds surviving over time (Figure 7a, Page 26).

\begin{tabular}{cccc}
\hline & & Fast Pathway & Slow Pathway \\
\cline { 3 - 4 } & $\mathrm{B}_{1}{ }^{0}$ & $\lambda_{1}, \mathrm{~s}^{-1}$ & $\lambda_{2}, \mathrm{~s}^{-1}$ \\
\hline $2-4 \mathrm{pN}$ & 0.82 & 24 & 2.9 \\
\hline $8-10 \mathrm{pN}$ & 0.63 & 66 & 6.9
\end{tabular}

Data are for the low ionic strength $(25 \mathrm{mM} \mathrm{KCl})$ condition with intact HMM loop 2. Errors have not been included because fits were based on a weighted regression, which invalidates the error estimates. Data were fit using Equation 15.

Table 6: The fitted parameters for the fraction of actin-HMM bonds in the short-lived pathway over load (Figure 7b, Page 26).

\footnotetext{
$\Delta \mathrm{E}, 10^{-21} \mathrm{~J} \quad \mathrm{X}_{\mathrm{i} \delta}, \AA$

$1.8 \pm 0.2 \quad 2.7 \pm 0.5$

Data are for low ionic strength $(25 \mathrm{~m} M \mathrm{KCl})$ condition with intact loop 2. Data were fit using Equation

2. Data are mean \pm standard error.
} 
Table 7: The fitted parameters of the reverse Bell model for actin-HMM specific 2D on-rates vs compressive load (Figure 8, 29).

\begin{tabular}{llcc}
\hline \multirow{2}{*}{ Ionic Strength $(\mathrm{mM})$} & Loop 2 & $\mathrm{k}_{\mathrm{on}}{ }^{0}, 10^{-5} \mu \mathrm{m}^{2} / \mathrm{s}$ & $\mathrm{x}$ \\
\hline 25 & Intact & $6.0 \pm 1.2$ & $0.20 \pm 0.07$ \\
\cline { 2 - 4 } & Cleaved & $4.2 \pm 0.8$ & $0.21 \pm 0.07$ \\
\hline & Intact & $5.6 \pm 1.2$ & $0.19 \pm 0.07$ \\
\cline { 2 - 4 } & Cleaved & $3.5 \pm 0.5$ & $0.13 \pm 0.06$
\end{tabular}

Data are for low and high ionic strength conditions with loop 2 intact and cleaved. Data were fit using Equation 16. Data are mean \pm standard error.

Table 8: The fitted parameters of the adhesion probability between actin and N-terminal cMyBP-C over a range of contact times (Figure 22, Page 57).

\begin{tabular}{lcc}
\hline cMyBP-C Fragment & $\mathrm{A}_{\mathrm{c}} \mathrm{m}_{\mathrm{r}} \mathrm{m}_{\mathrm{l}} \mathrm{k}_{\mathrm{f}}{ }^{1}, 10^{-3} \mathrm{~ms}^{-1}$ & $\mathrm{k}_{\mathrm{r}}{ }^{0}, 10^{-3} \mathrm{~ms}^{-1}$ \\
\hline C0C3 & $2.4 \pm 0.7$ & $2.9 \pm 0.6$ \\
\hline C0C3-4D & $2.3 \pm 0.6$ & $2.7 \pm 0.9$ \\
\hline
\end{tabular}

Data were fit using Equation 7. Data are mean \pm standard error. 
Table 9: The fitted parameters of the single bound pathway dissociation model for actin- $\mathrm{N}$-terminal cMyBP-C bond lifetime vs load data (Figure 19, Page 52)

\begin{tabular}{lcc}
\hline cMyBP-C Fragment & $\mathrm{k}_{\mathrm{s}}{ }^{0}, \mathrm{~s}^{-1}$ & $\mathrm{x}_{\mathrm{s}}, \mathrm{nm}$ \\
\hline C0C3 & $6.8 \pm 0.7$ & $0.15 \pm 0.03$ \\
\hline C0C3-4D & $6.5 \pm 0.07$ & $0.19 \pm 0.03$ \\
\hline
\end{tabular}

Data were fit using Equation 6. Data are mean \pm standard error.

Table 10: The fitted parameters of the single bound pathway dissociation model for HMM-N-terminal cMyBP-C bond lifetime vs load data (Figure 20, Page 54).

\begin{tabular}{llc}
\hline cMyBP-C Fragment & $\mathrm{k}_{\mathrm{s}}{ }^{0}, \mathrm{~s}^{-1}$ & $\mathrm{x}_{\mathrm{s}}, \mathrm{nm}$ \\
\hline C0C3 & $6.4 \pm 0.8$ & $0.16 \pm 0.04$ \\
\hline C0C3-4D & $6.6 \pm 0.7$ & $0.21 \pm 0.04$ \\
\hline
\end{tabular}

Data were fit using Equation 6. Data are mean \pm standard error.

Table 11: The fitted parameters of actin-HMM motility velocity over a range of COC3 concentrations (Figure 24, Page 60)

\begin{tabular}{lcc}
\hline Inhibitor & $\mathrm{k}_{\mathrm{i}, \mu \mathrm{M}}$ & $\mathrm{n}$ \\
\hline C0C3 & 0.8 & 0.7
\end{tabular}

Data were fit using Equation 9. Errors have not been included because the number of data points was not greater than the number of fitted parameters. 
Table 12: The fitted parameters of actin-HMM motility velocity over a range of cMyBP-C concentrations (Figure 25, Page 73).

\begin{tabular}{lcc}
\hline Inhibitor & $\mathrm{k}_{\mathrm{i}}(\mu \mathrm{M})$ & $\mathrm{n}$ \\
\hline cMyBP-C & $0.4 \pm 0.1$ & $0.5 \pm 0.1$ \\
\hline Nitrosylated cMyBP-C & $0.6 \pm 0.4$ & $0.5 \pm 0.2$ \\
\hline
\end{tabular}

Data were fit using Equation 9. Data are mean \pm standard error. 
Appendix 3: Matlab Code 


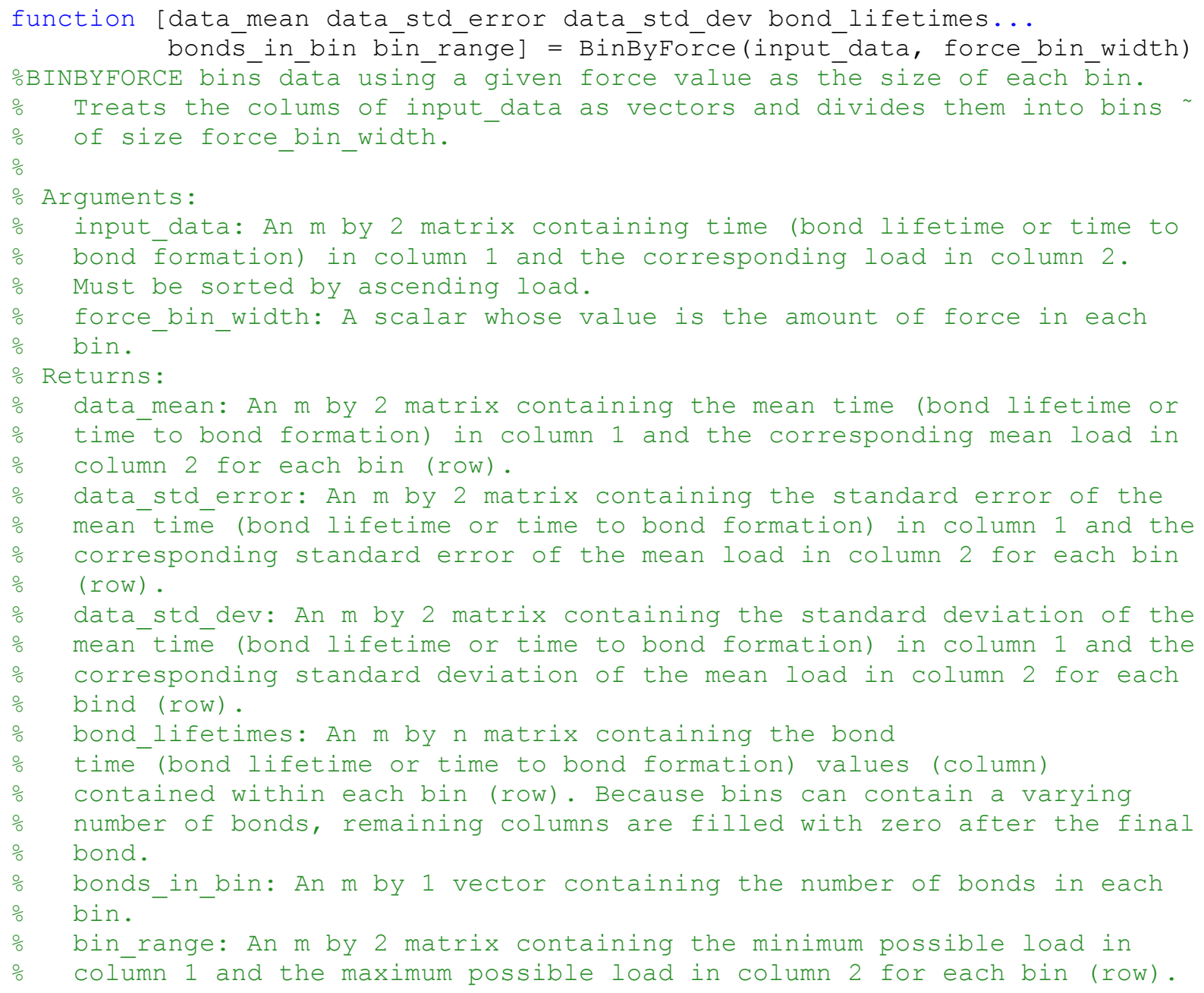




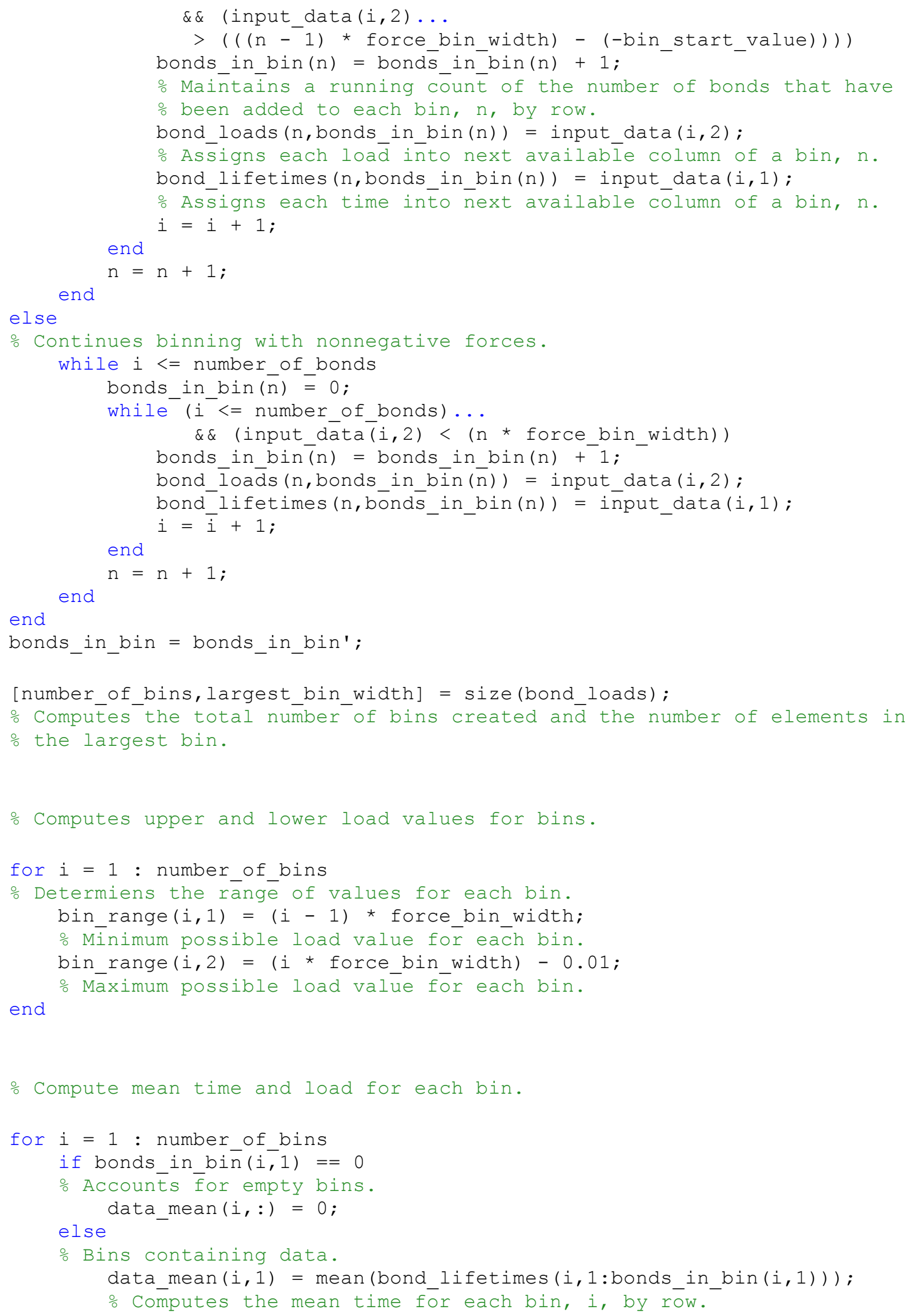




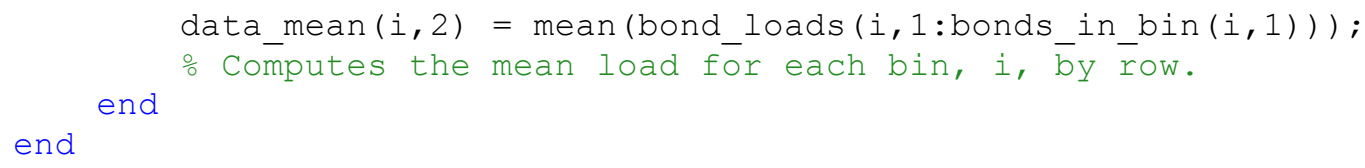

end 


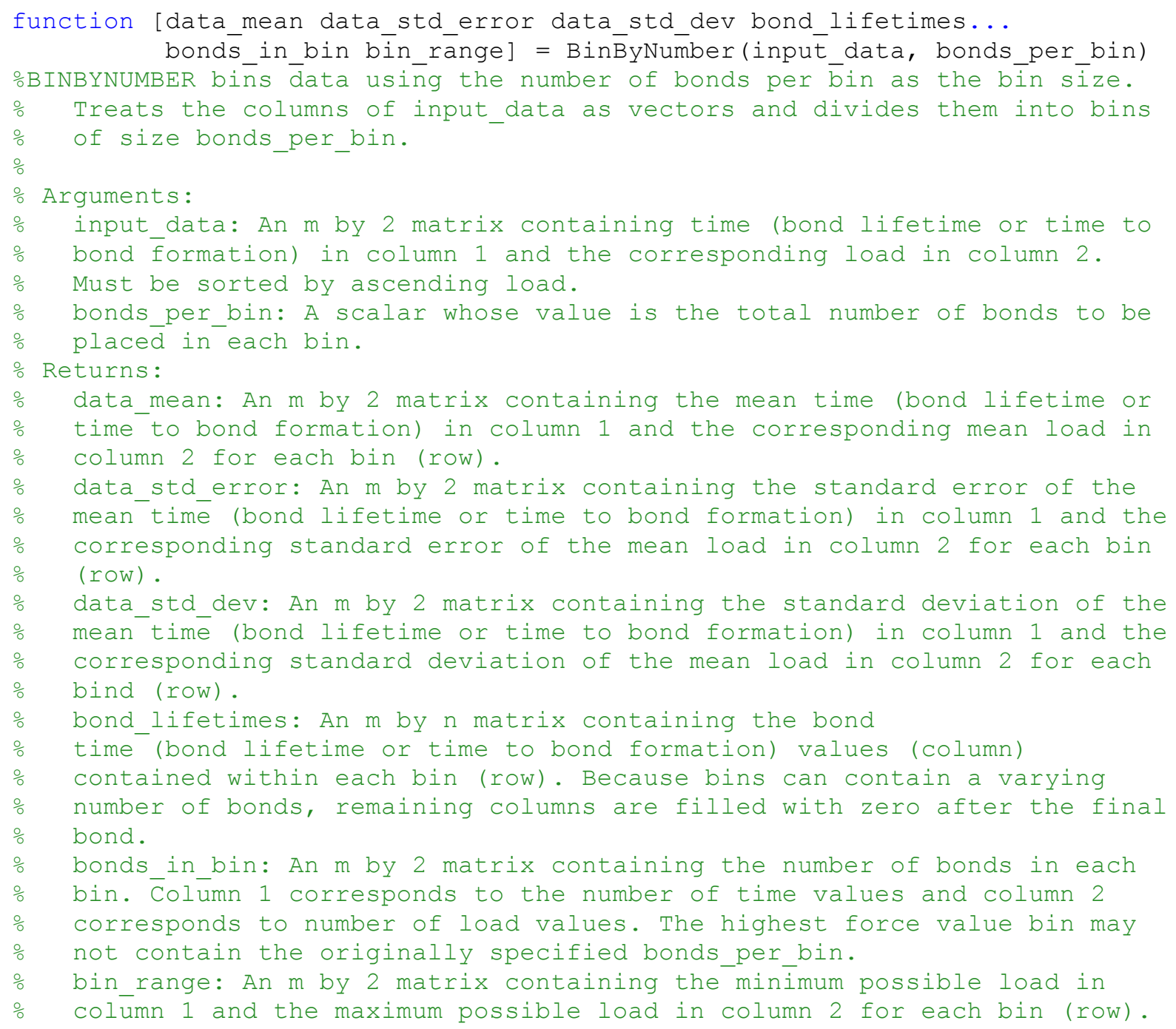

응 Determine number of bins needed.

number_of_bonds = length (input_data);

\% Computes the total number of bonds in data set. 


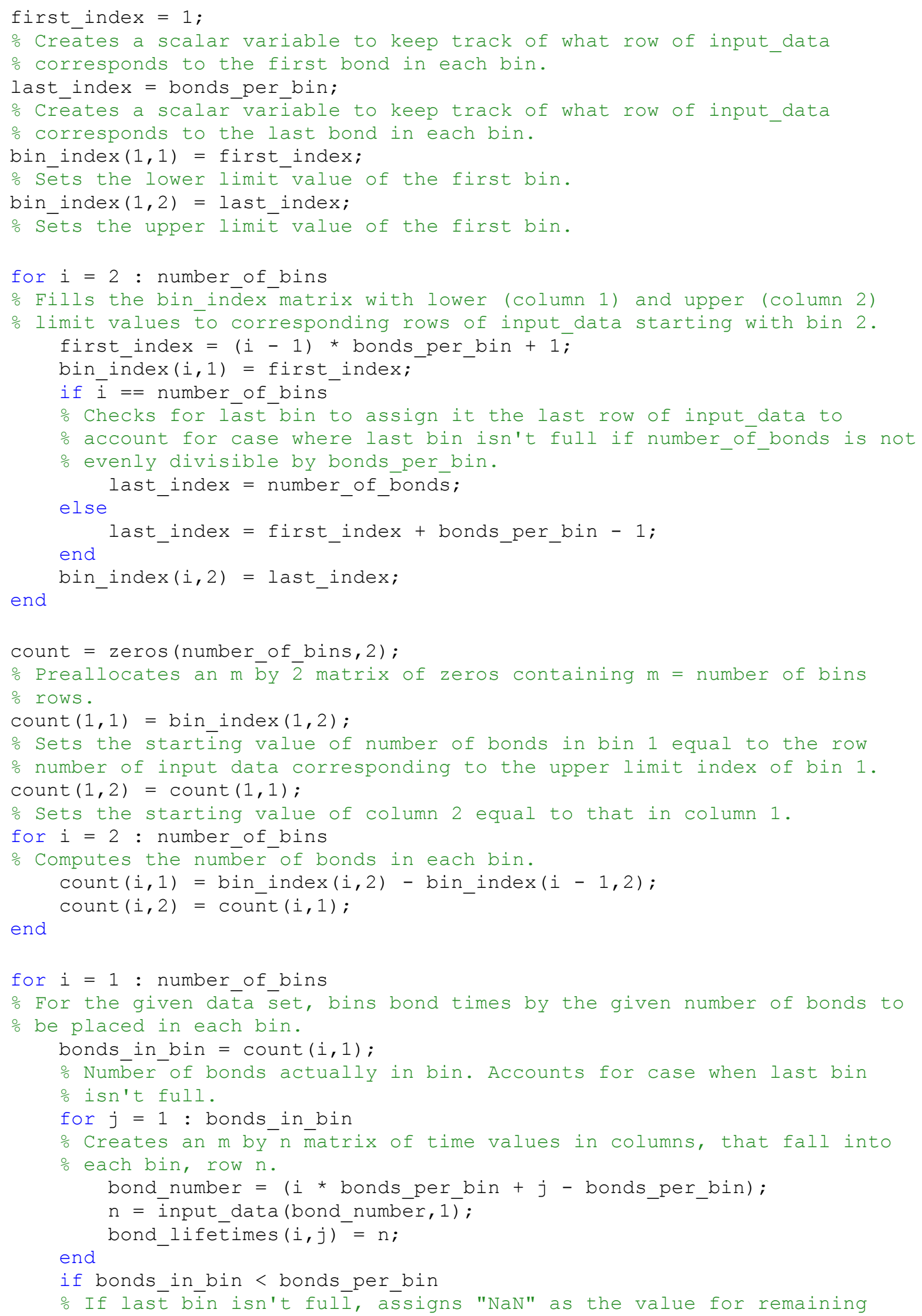




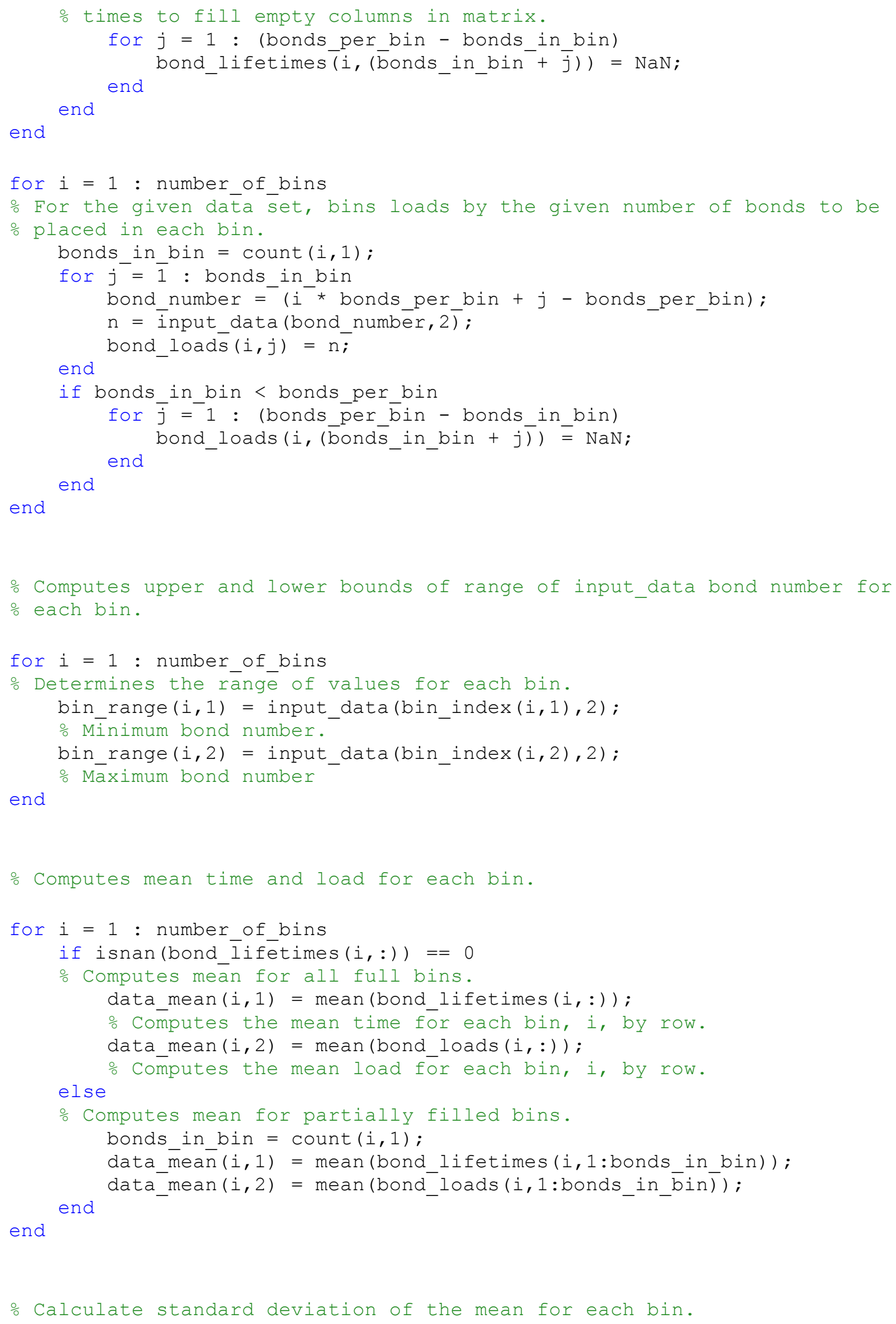




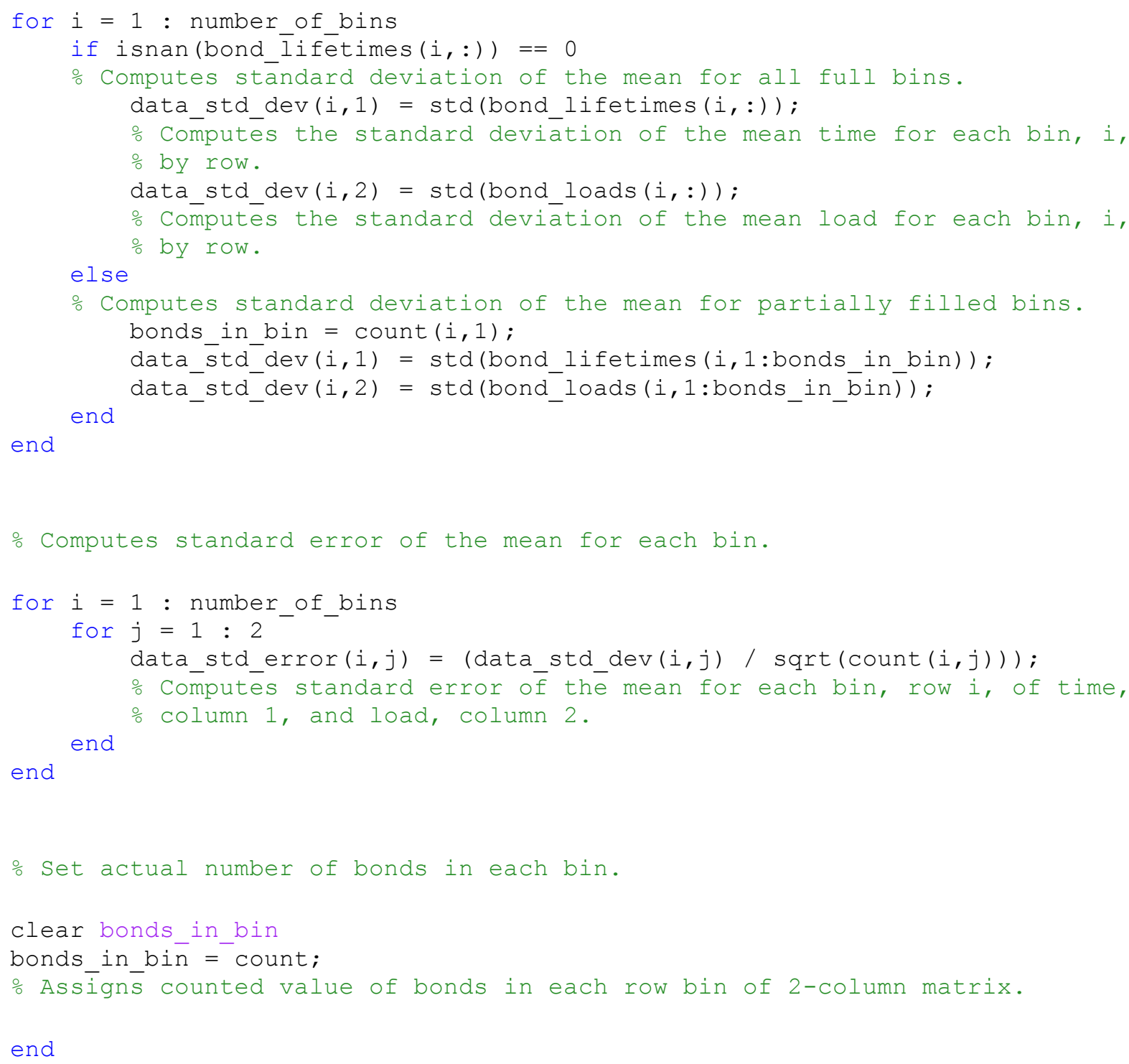




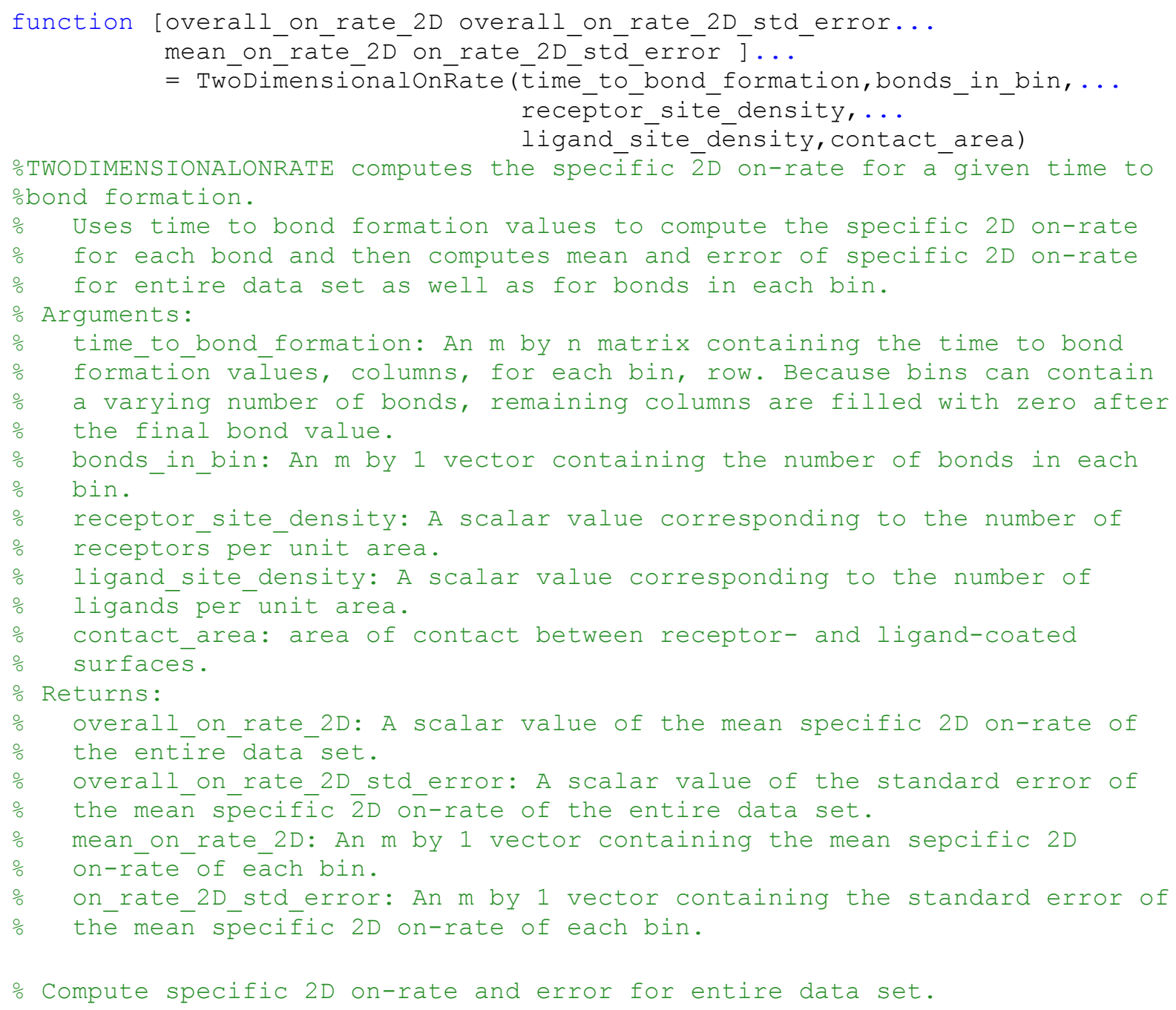




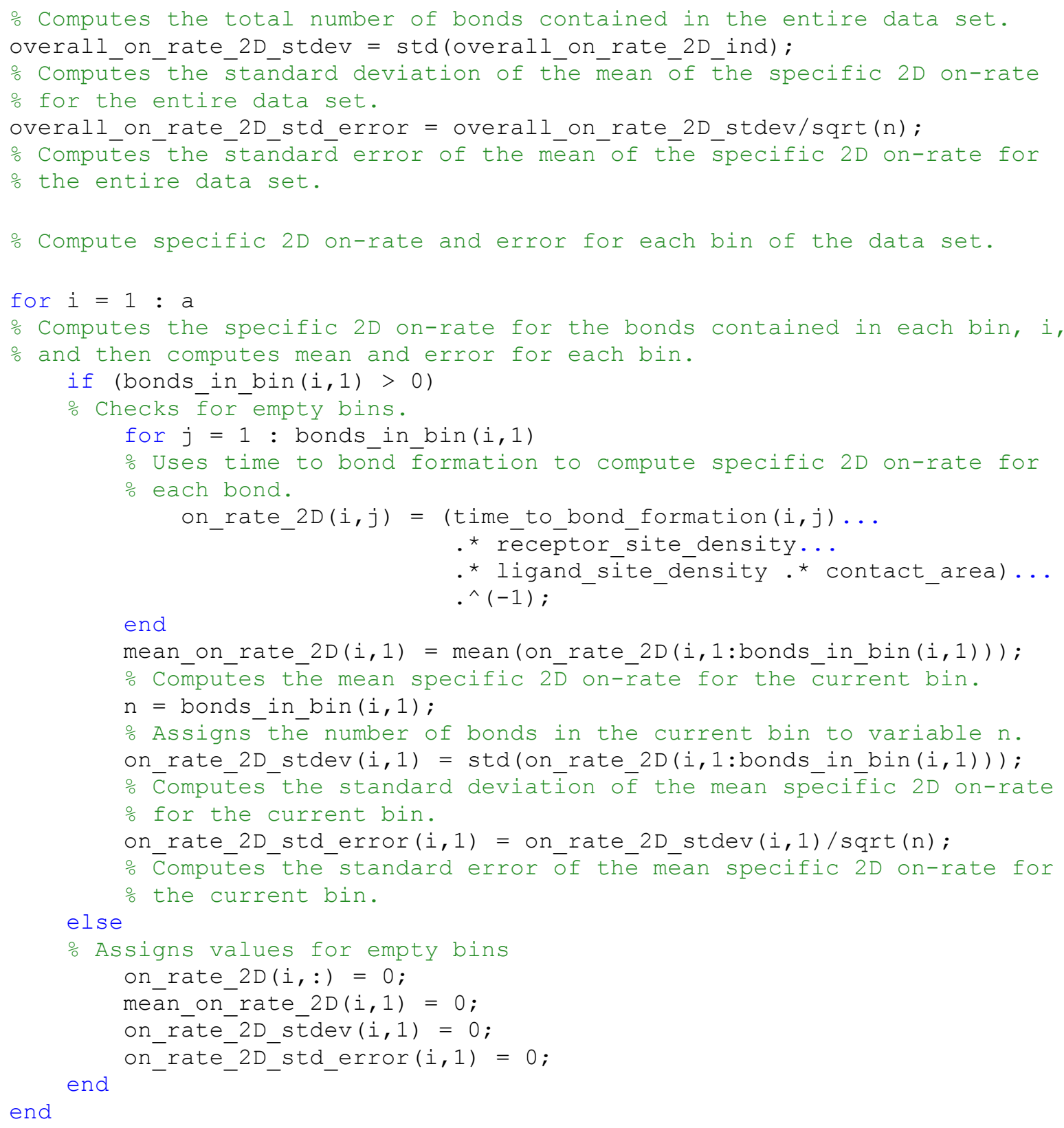




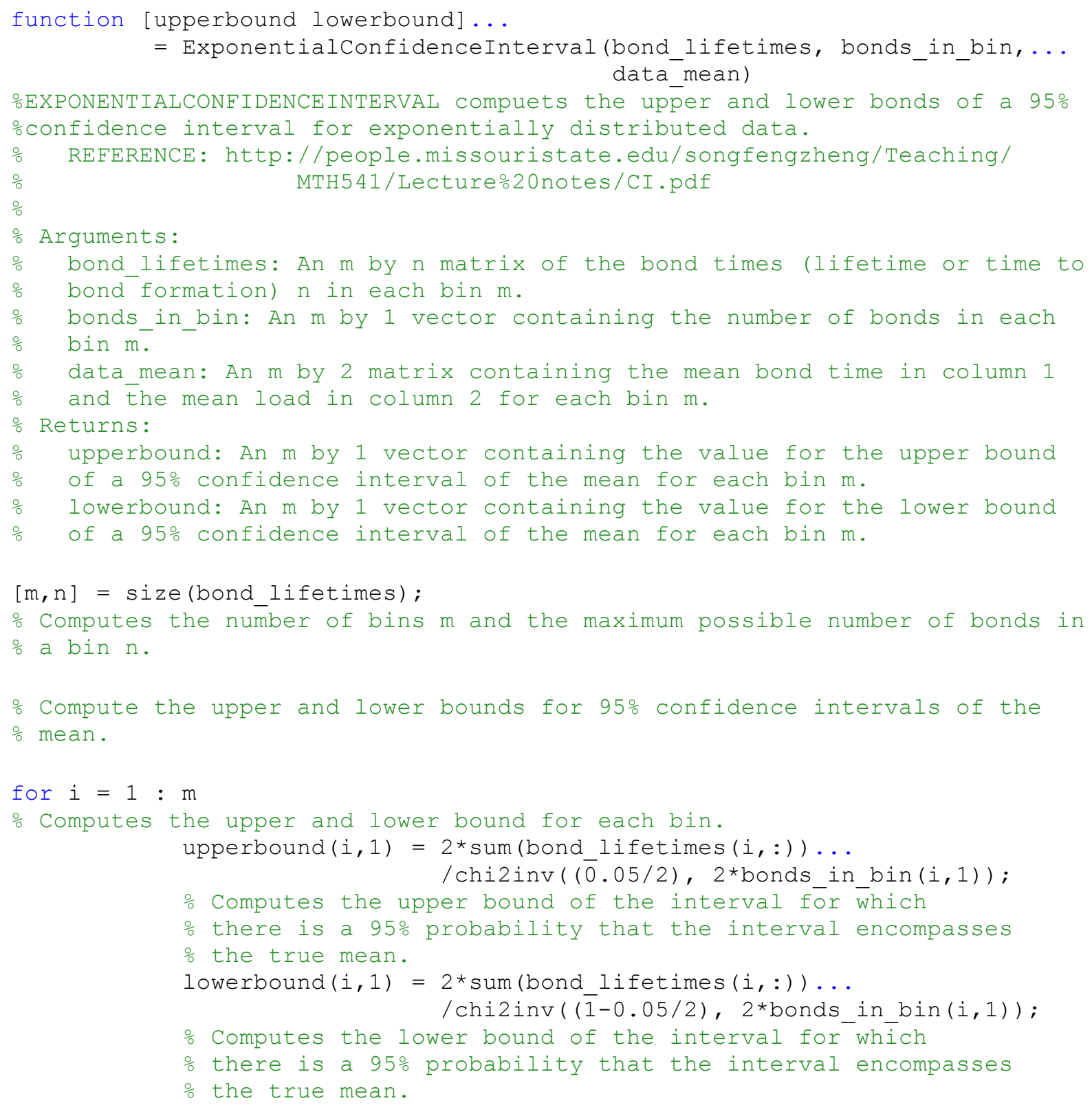

end 


\section{Appendix 4: Matlab Code for Cycling Crossbridge Model}


\% Skeletal and Smooth crossbridge rates functions used to make supplemental

o figures for paper.

\% TIME TO DETACHMENT

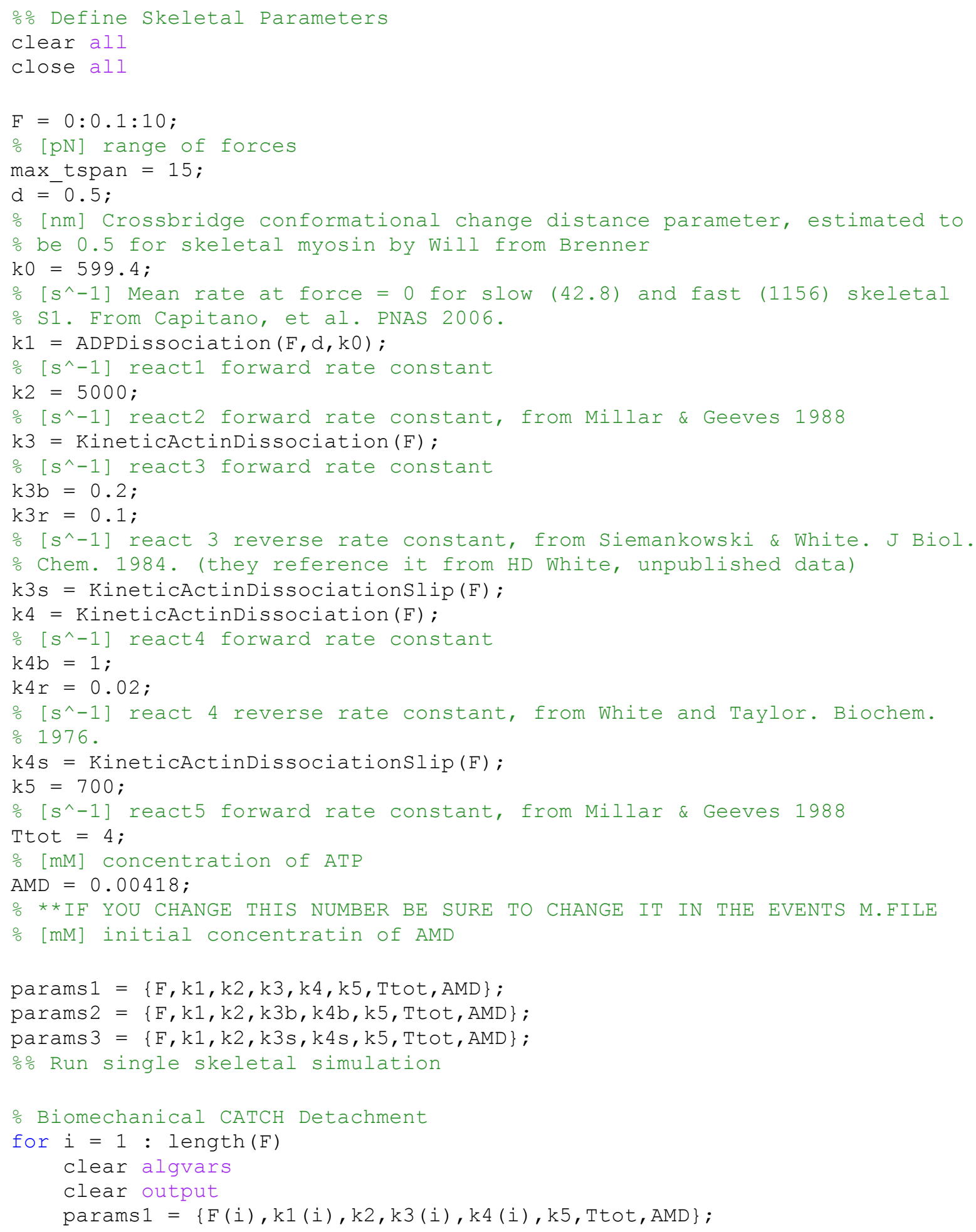




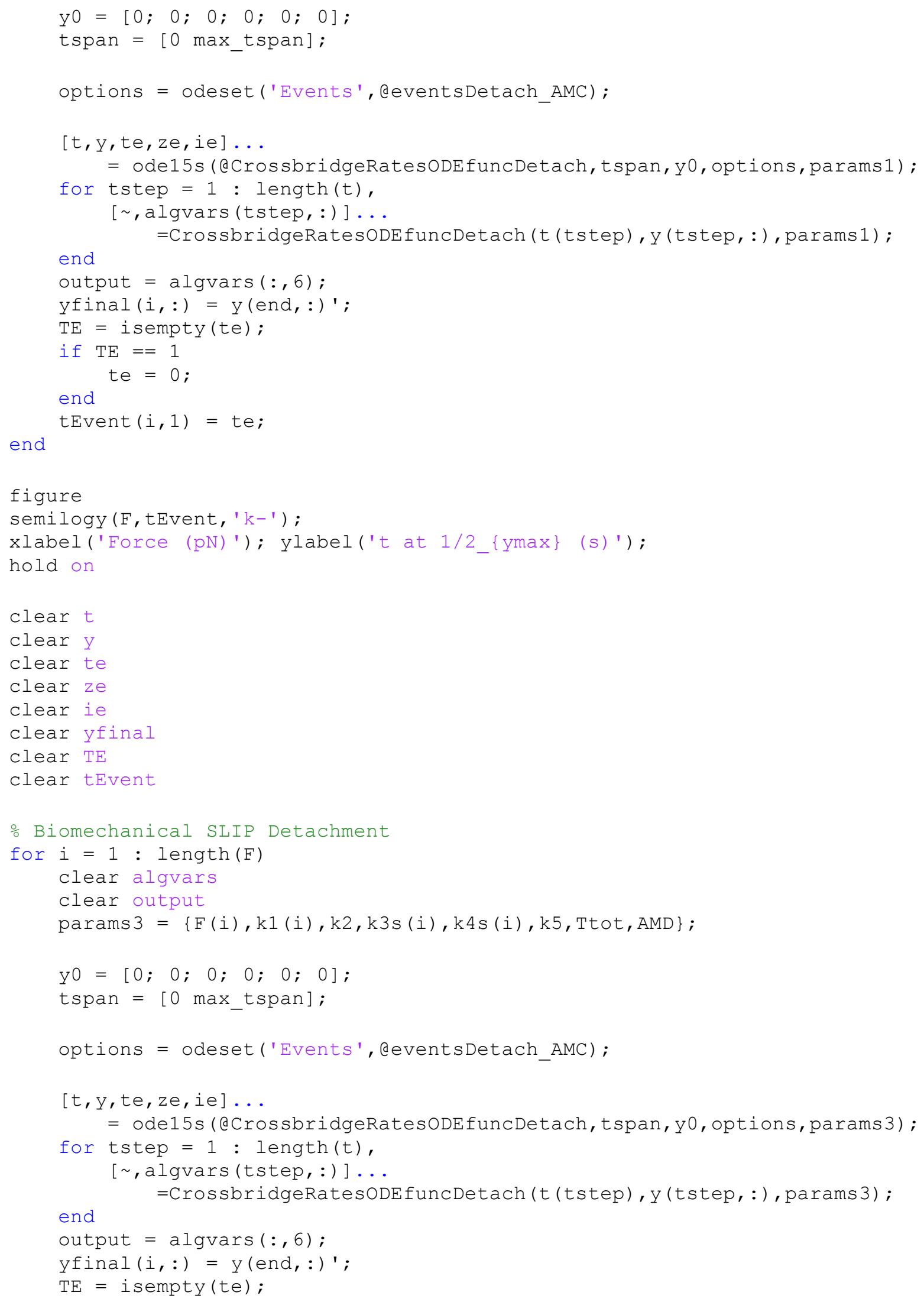




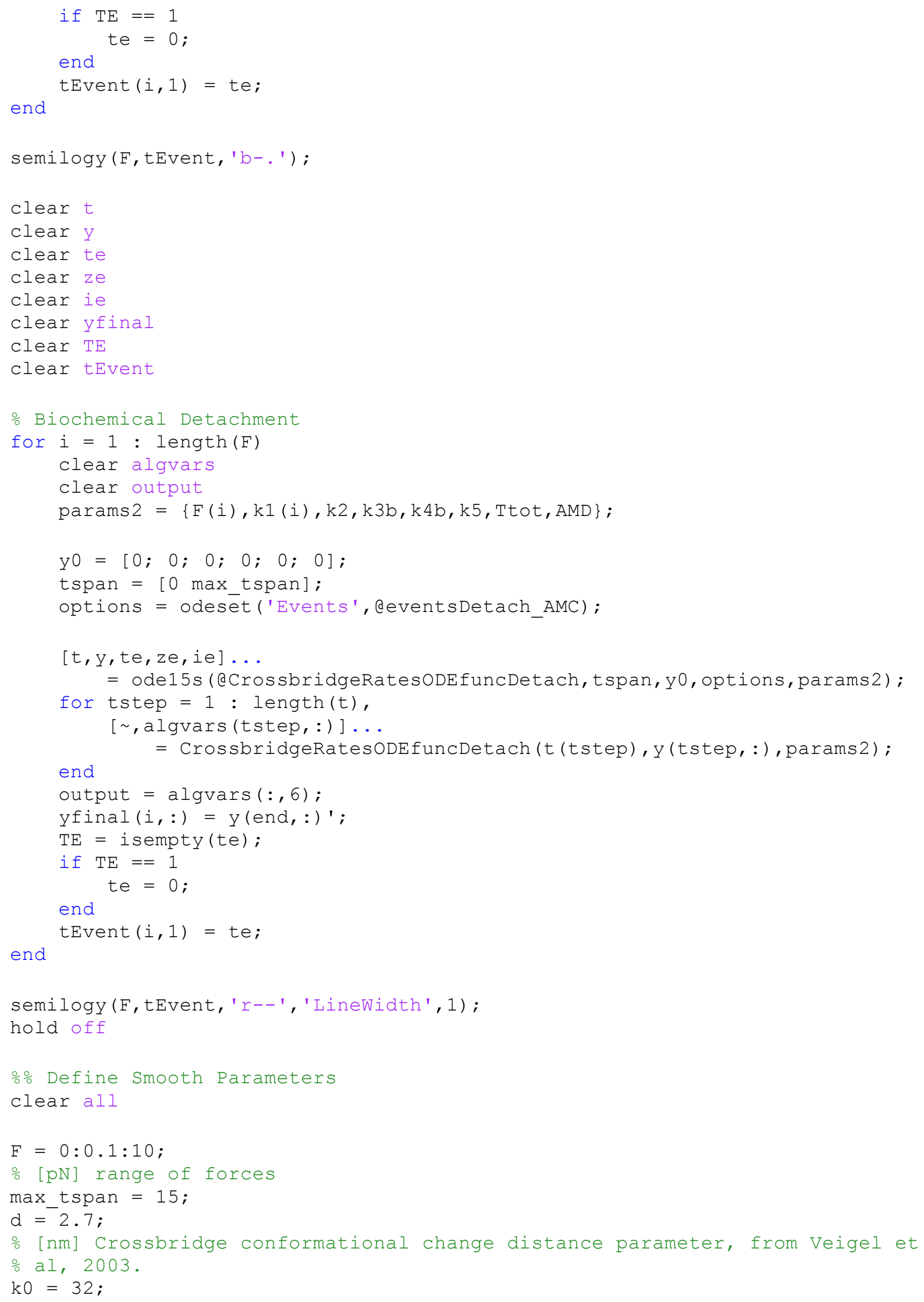


\% [s^-1] Unloaded on-rate for ADP dissociation from actomyosin, from Veigel

을 al 2003 .

$\mathrm{k} 1=\operatorname{ADPDissociation}(\mathrm{F}, \mathrm{d}, \mathrm{k} 0)$;

$\circ\left[\mathrm{s}^{\wedge}-1\right]$ react 1 forward rate constant

$\mathrm{k} 25=380$;

\% $\left[\mathrm{S}^{\wedge}-1\right]$ react $2 / 5$ forward rate constant, calculated from

ㅇ.510^4 M-1s-1 * $4 \mathrm{mM}$ [ATP], from Khromov, Somlyo, and Somlyo. J. Phil. 응 1996 .

$\mathrm{k} 3 \mathrm{~b}=0.0035 ;$

\% $\left[\mathrm{s}^{\wedge}-1\right]$ react3 forward rate constant, from Cremo \& Geeves, Biochemistry, $\div 1998$

$\mathrm{k} 3=$ KineticActinDissociation (F);

$\div\left[\mathrm{S}^{\wedge}-1\right]$ react 3 forward rate constant

$\mathrm{k} 3 \mathrm{~s}=$ KineticActinDissociationslip (F);

$\mathrm{k} 4 \mathrm{~b}=0.004 ;$

\% $\left[\mathrm{s}^{\wedge}-1\right]$ react4 forward rate constant, from Cremo \& Geeves, Biochemistry, $\div 1998$

$\mathrm{k} 4$ = KineticActinDissociation $(\mathrm{F})$;

$\div\left[\mathrm{s}^{\wedge}-1\right]$ react 4 forward rate constant

$\mathrm{k} 4 \mathrm{~s}=$ KineticActinDissociationslip $(\mathrm{F})$;

$\mathrm{k} 6=1.9$;

\% $\left[\mathrm{s}^{\wedge}-1\right]$ react 6 forward rate constant, from Cremo \& Geeves, Biochemistry, $\div 1998$

$\mathrm{k} 7=12.8 ;$

\% $\left[\mathrm{s}^{\wedge}-1\right]$ react 7 forward rate constant, from Cremo \& Geeves, Biochemistry, 응 1998

Ttot $=4$;

\% $[\mathrm{mM}]$ concentration of ATPAMD $=0.000352 ; \quad \% * *$ IF YOU CHANGE THIS NUMBER \% BE SURE TO CHANGE IT IN FILE EVENTS.M [mM] initial concentratin of AMD $\mathrm{AMD}=0.000352$;

\% **IF YOU CHANGE THIS NUMBER BE SURE TO CHANGE IT IN THE EVENTS M.FILE

$\circ[\mathrm{mM}]$ initial concentratin of AMD

params $1=\{\mathrm{F}, \mathrm{k} 1, \mathrm{k} 25, \mathrm{k} 3, \mathrm{k} 4, \mathrm{Ttot}, \mathrm{AMD}\}$

params $2=\{\mathrm{F}, \mathrm{k} 1, \mathrm{k} 25, \mathrm{k} 3 \mathrm{~b}, \mathrm{k} 4 \mathrm{~b}, \mathrm{Ttot}, \mathrm{AMD}\}$;

params $3=\{\mathrm{F}, \mathrm{k} 1, \mathrm{k} 25, \mathrm{k} 3 \mathrm{~s}, \mathrm{k} 4 \mathrm{~s}, \mathrm{Ttot}, \mathrm{AMD}\}$;

\% Run single smooth simulation

\% Biomechanical CATCH Dissociation

for $i=1$ : length $(F)$

clear algvars

clear output

params1 = $\{\mathrm{F}(\mathrm{i}), \mathrm{k} 1$ (i), k25, k3 (i), k4(i), Ttot, AMD $\}$;

$\mathrm{y} 0=[0 ; 0 ; 0 ; 0 ; 0]$;

tspan $=[0$ max tspan $]$

options = odesēt('Events', deventsSmoothDetach_AMC);

$[t, y, t e, z e, i e] \ldots$

= ode15s (@CrossbridgeRatesODEfuncSmoothDetach, tspan, y0, options,... params1);

for tstep $=1$ : length (t),

$[\sim$, algvars (tstep, :) ] ...

= CrossbridgeRatesODEfuncSmoothDetach (t (tstep), y (tstep, : ), .. params1); 


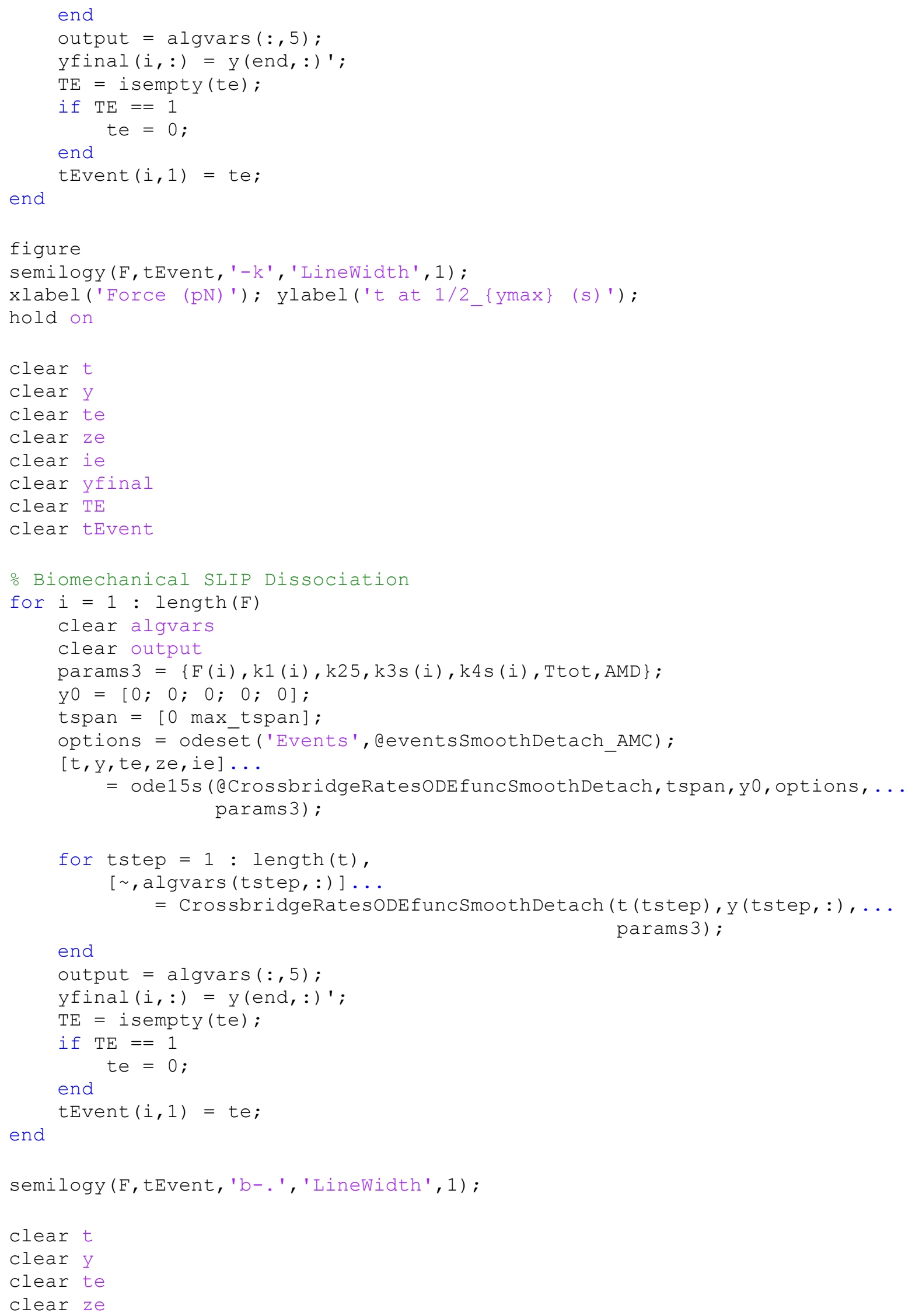




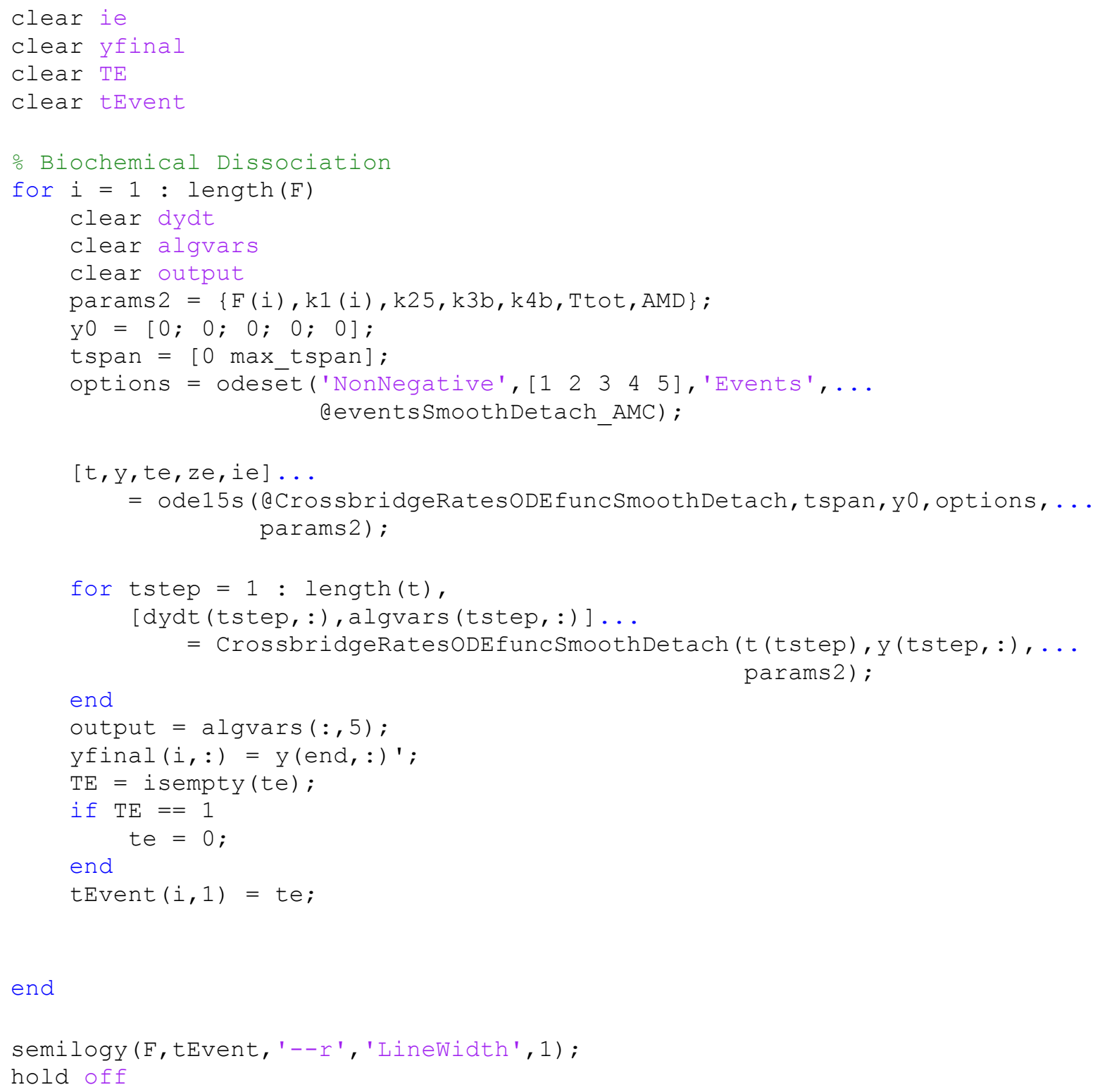


\% Skeletal and Smooth crossbridge rates functions used to make supplemental

o figures for paper. Includes a slip bond pathway biomechanical

o dissociation.

응 Cycle

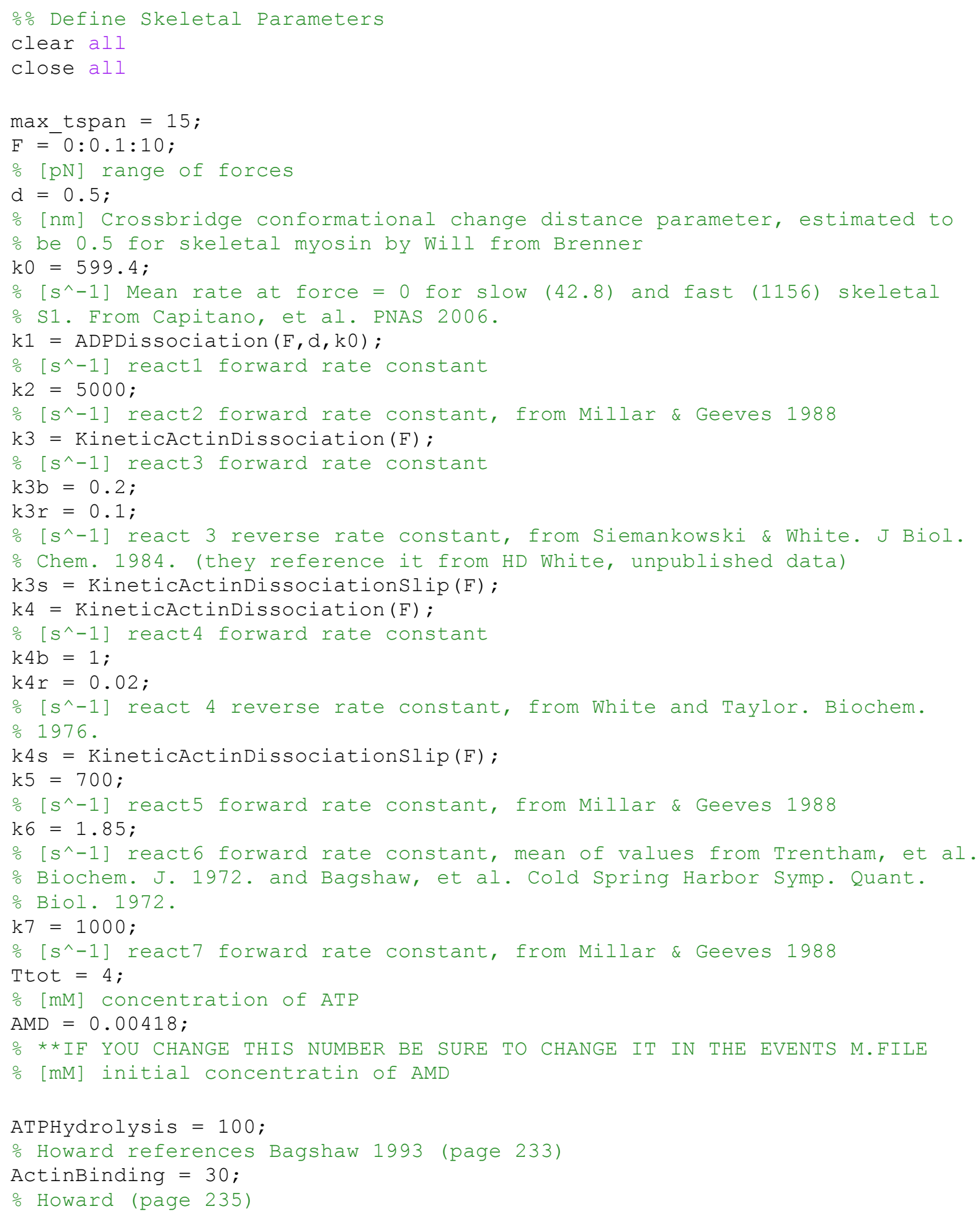




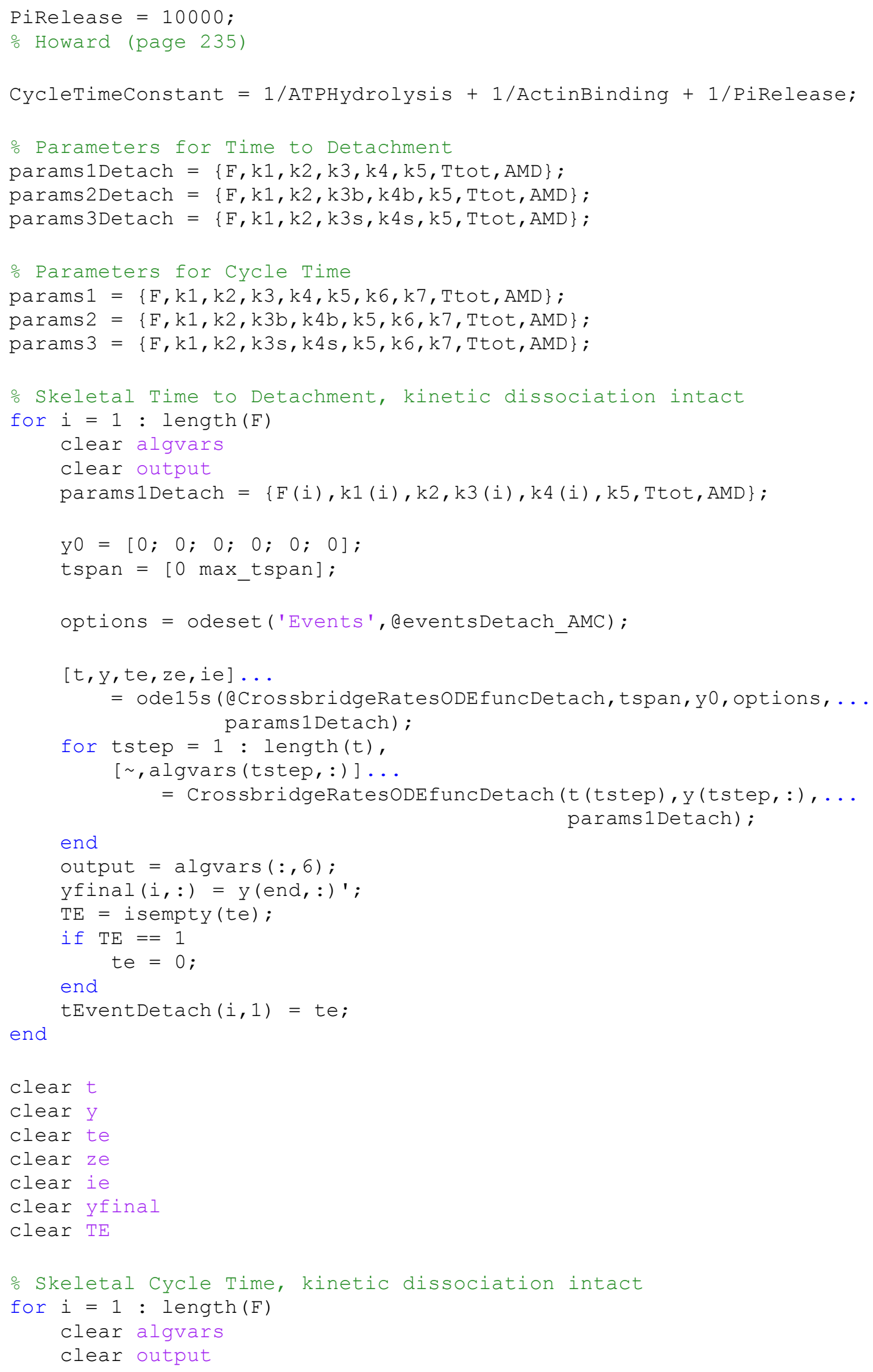




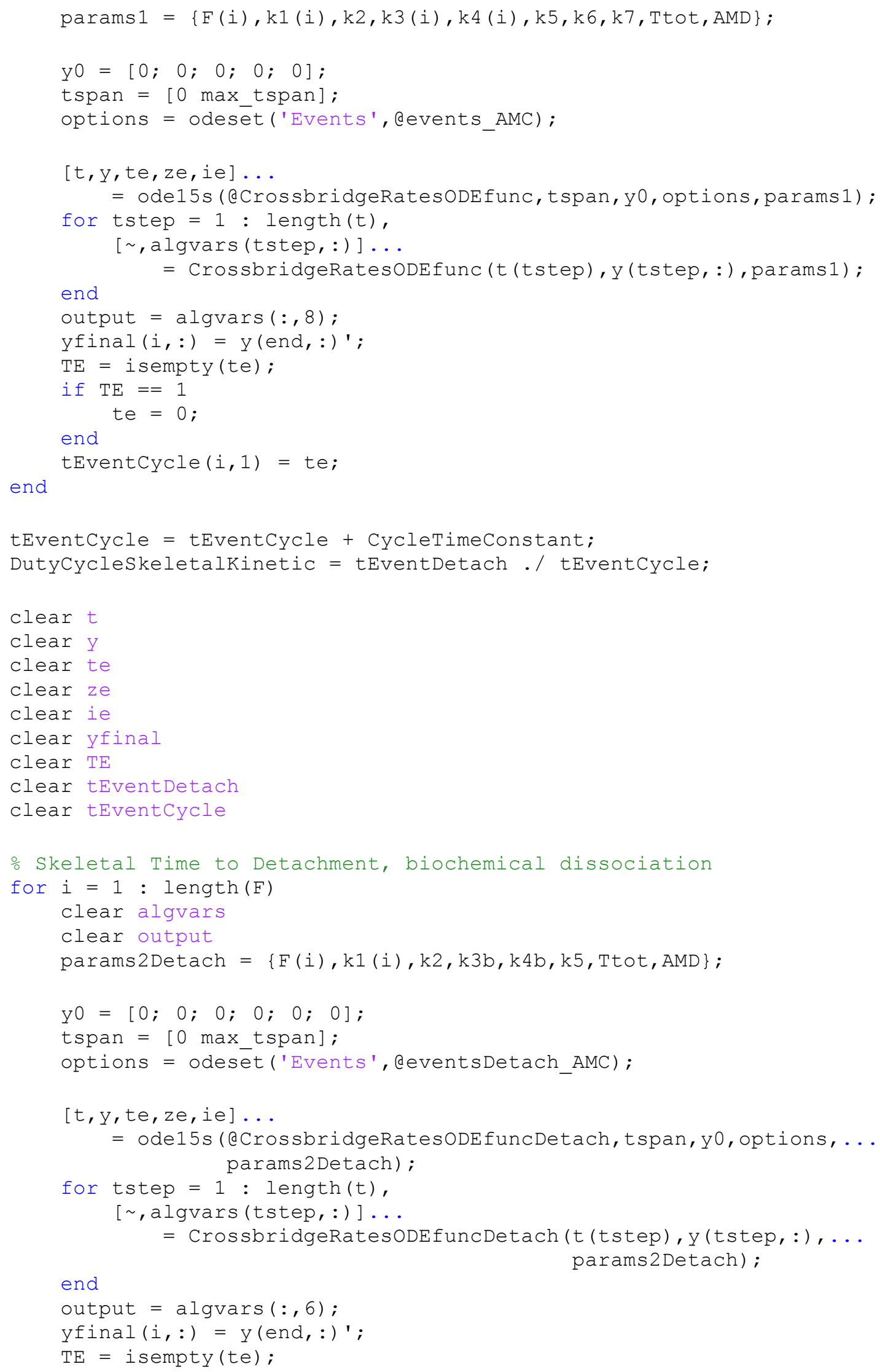




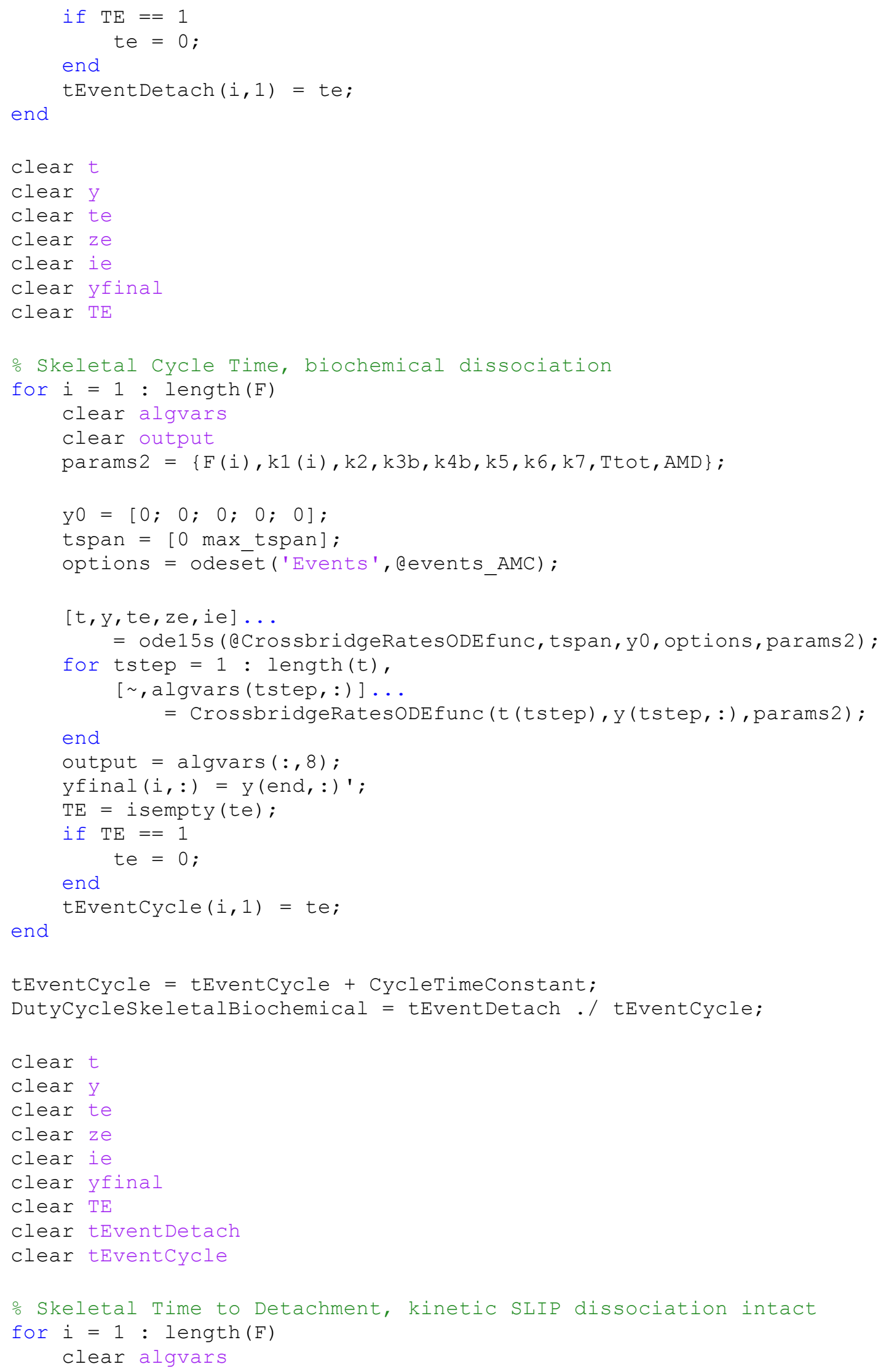




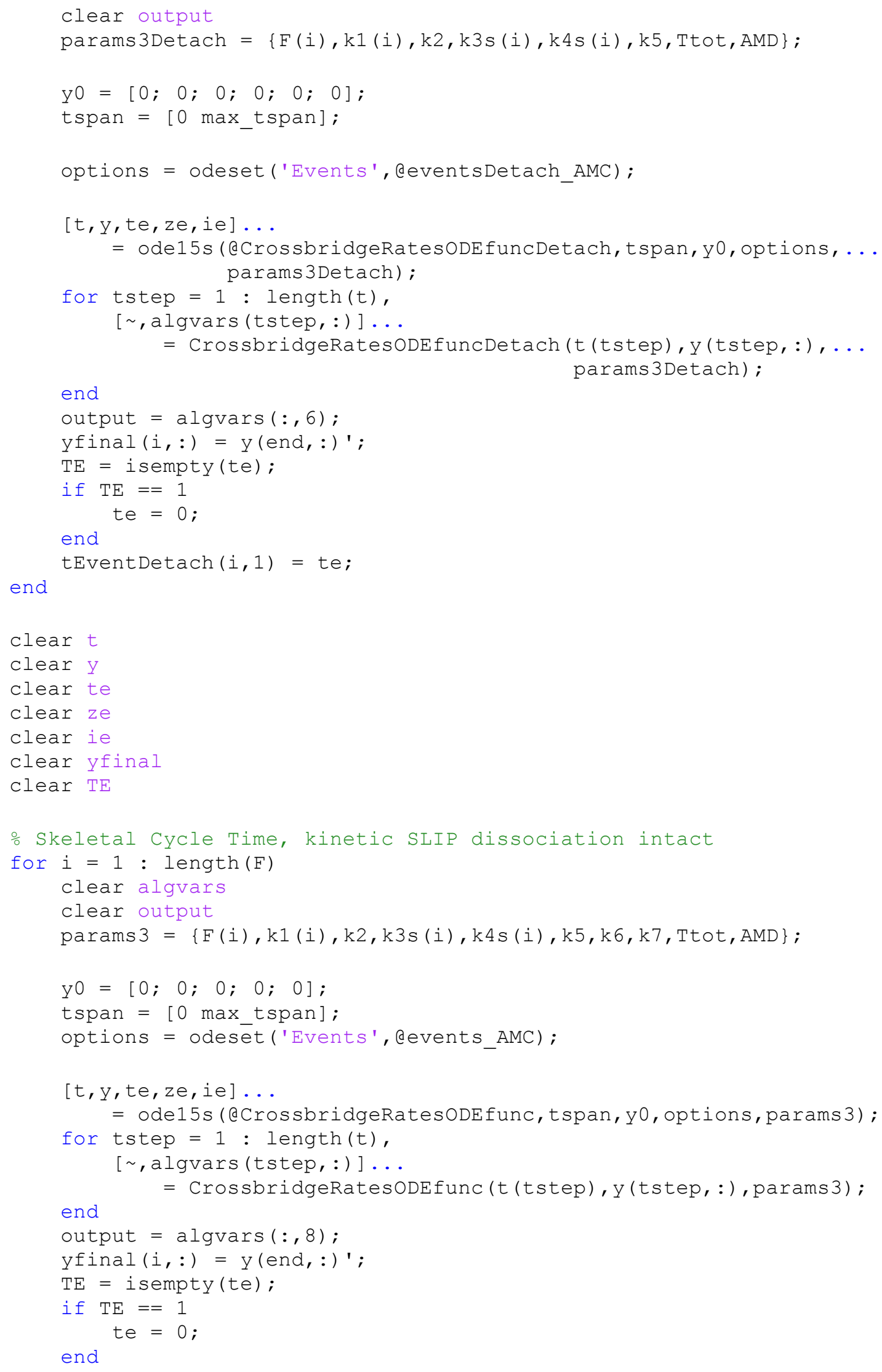




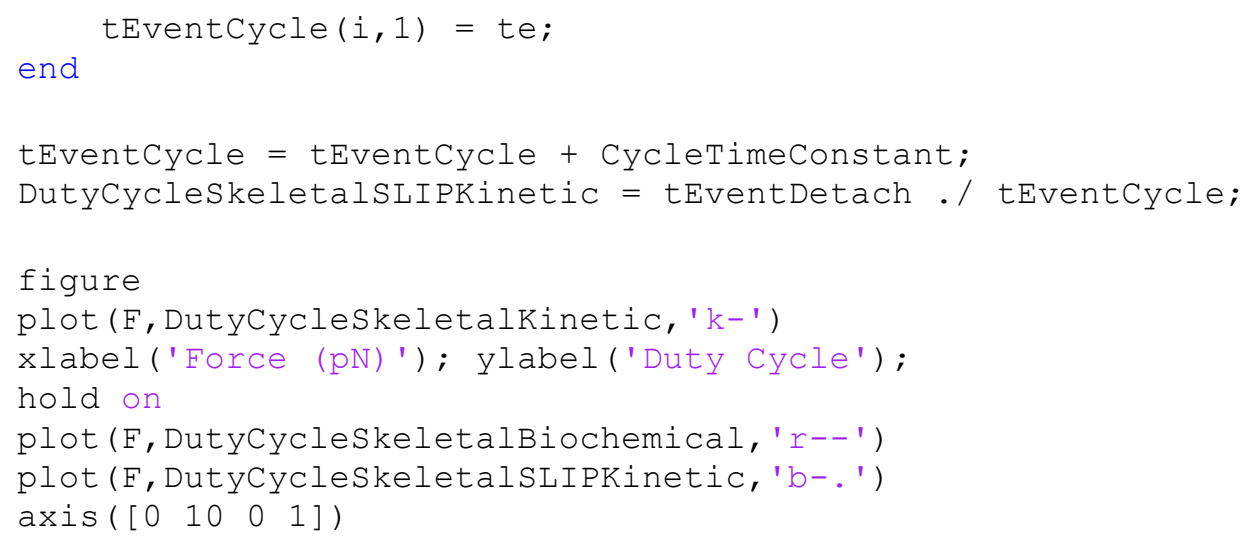




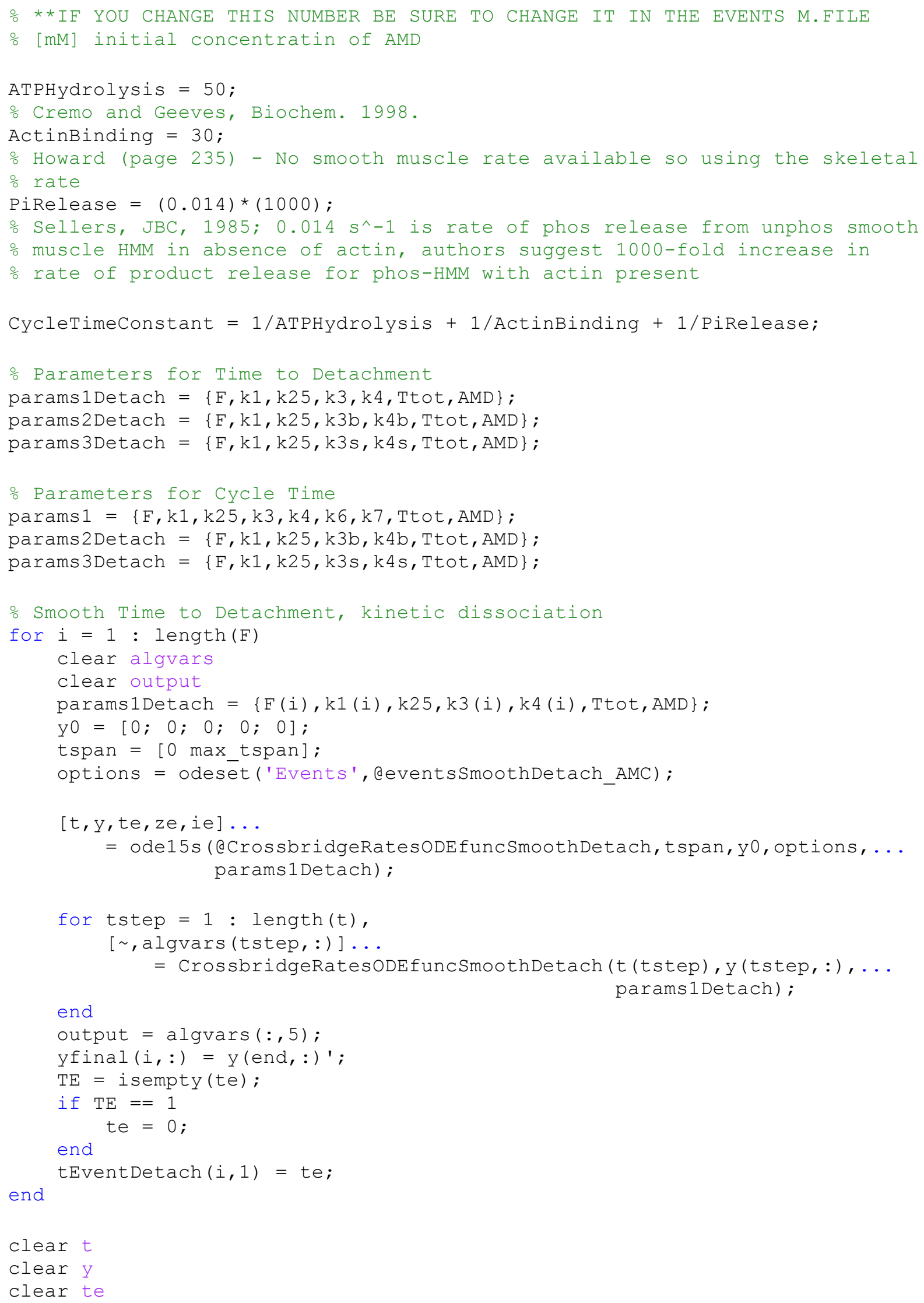




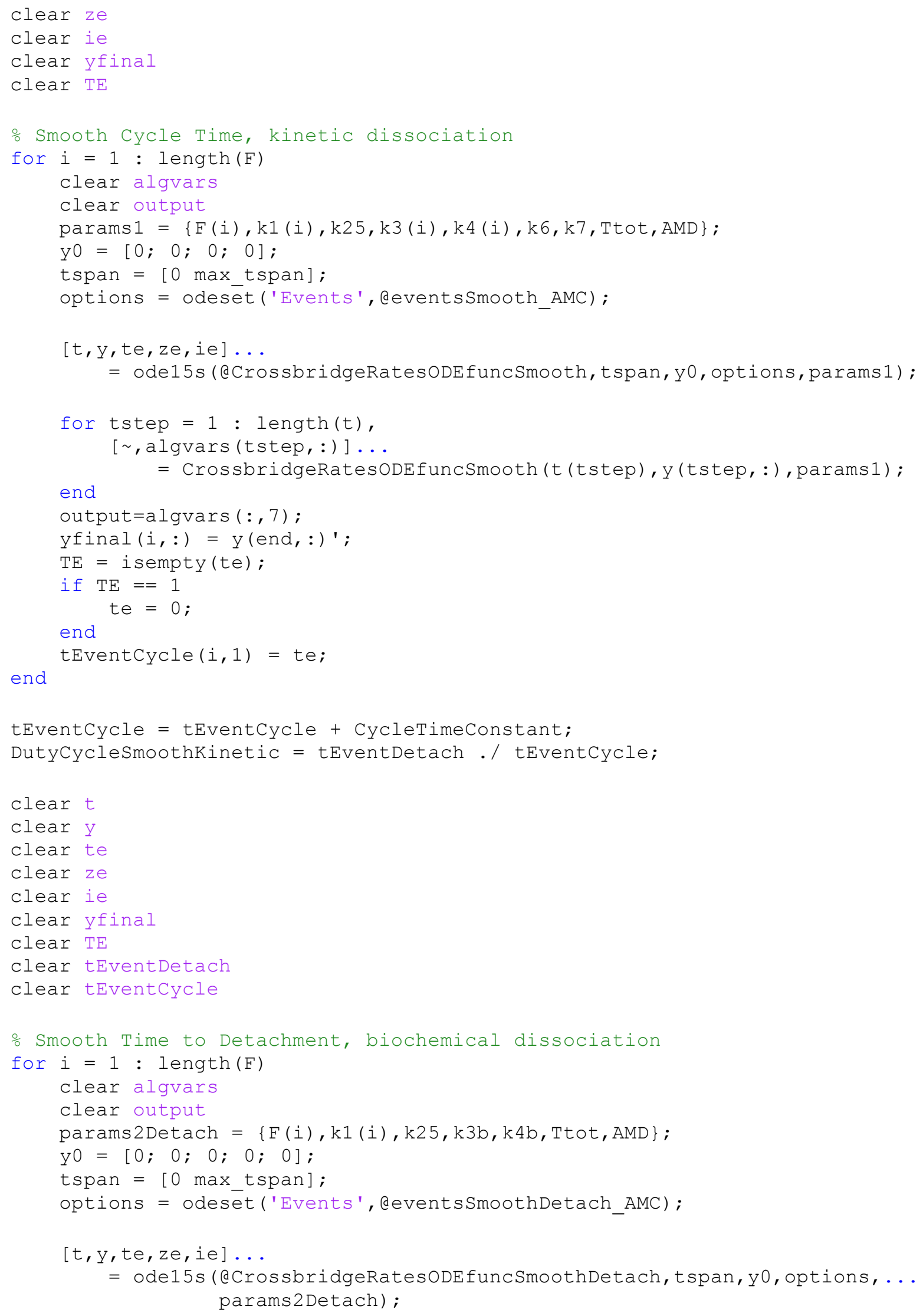




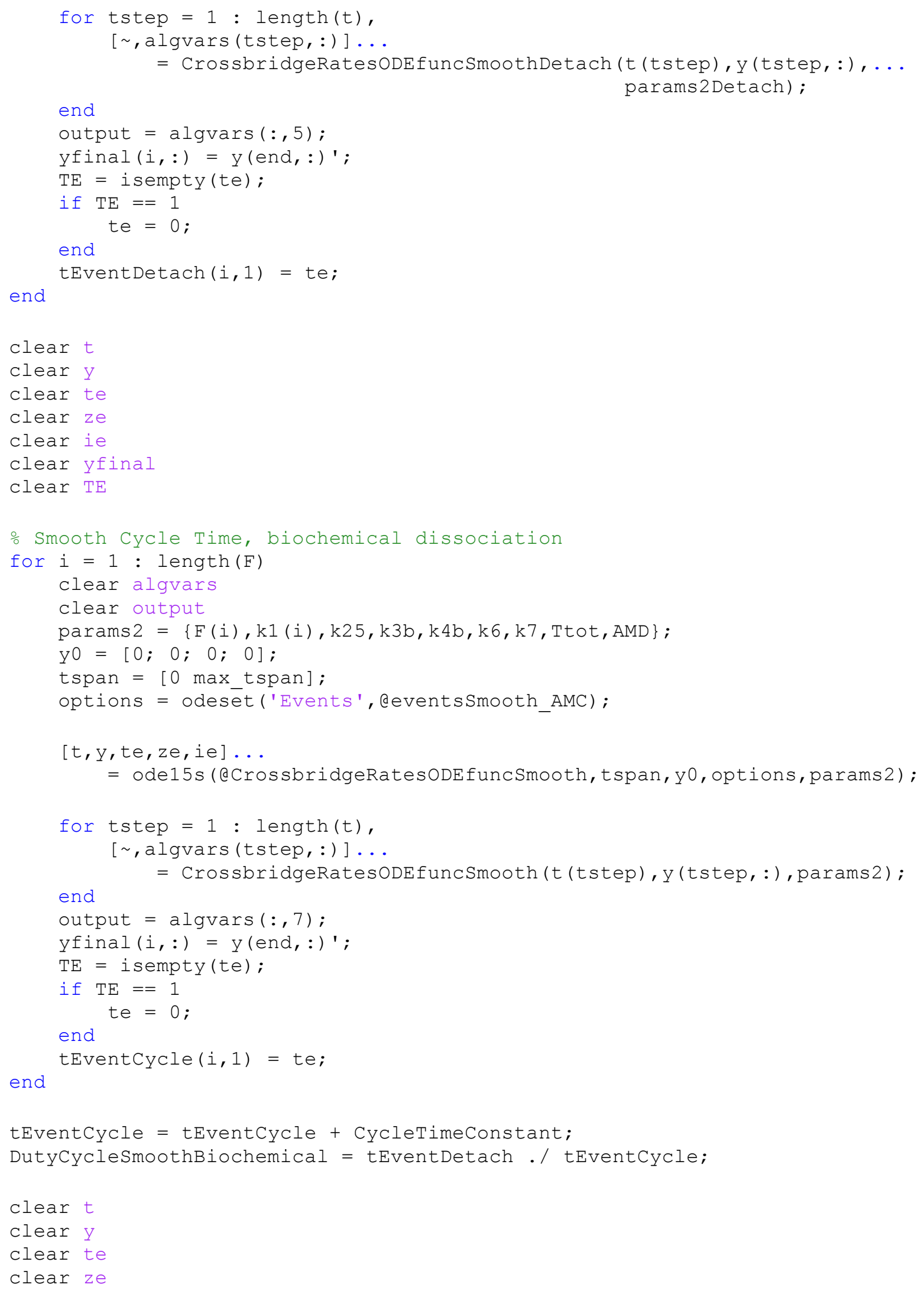




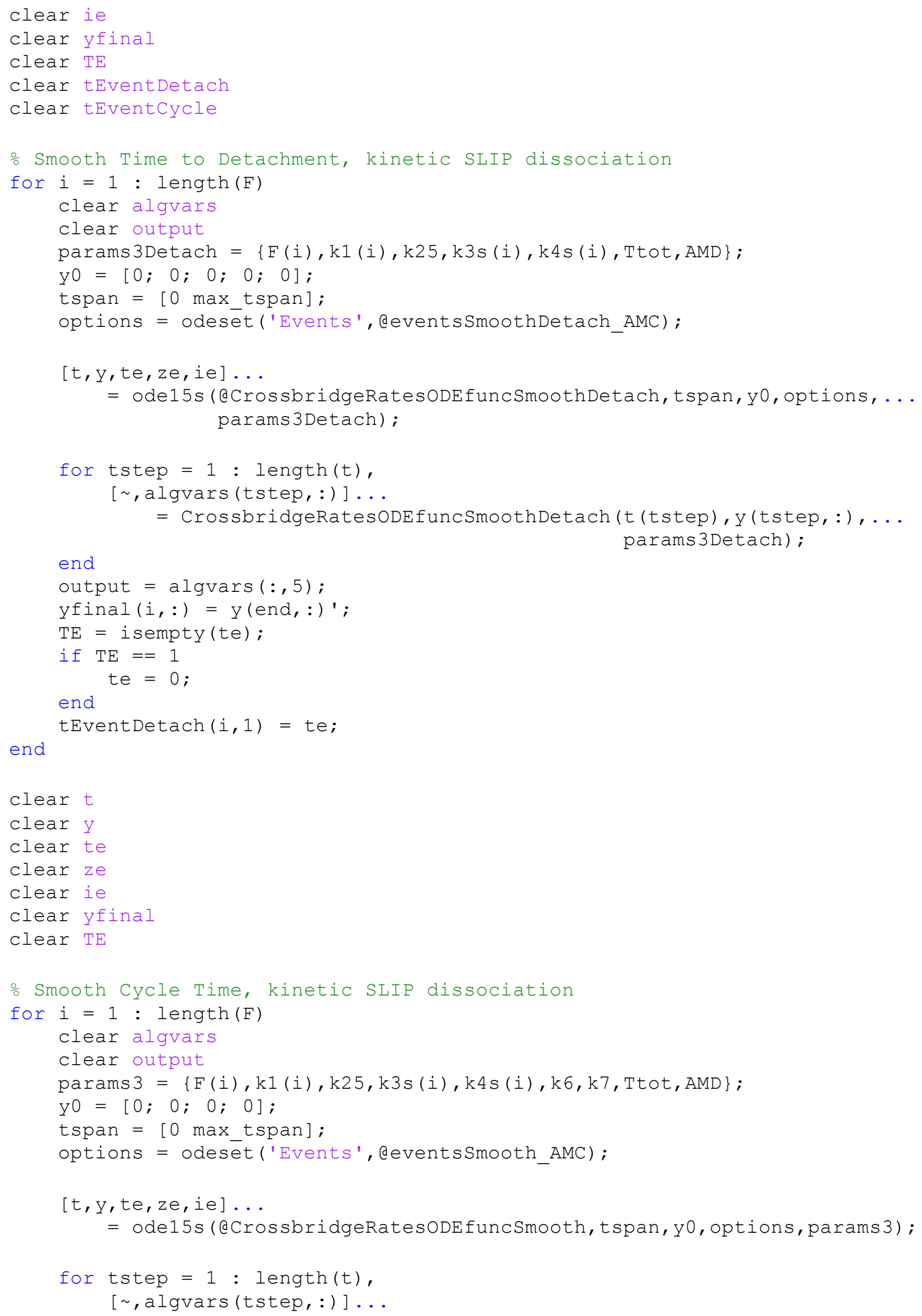




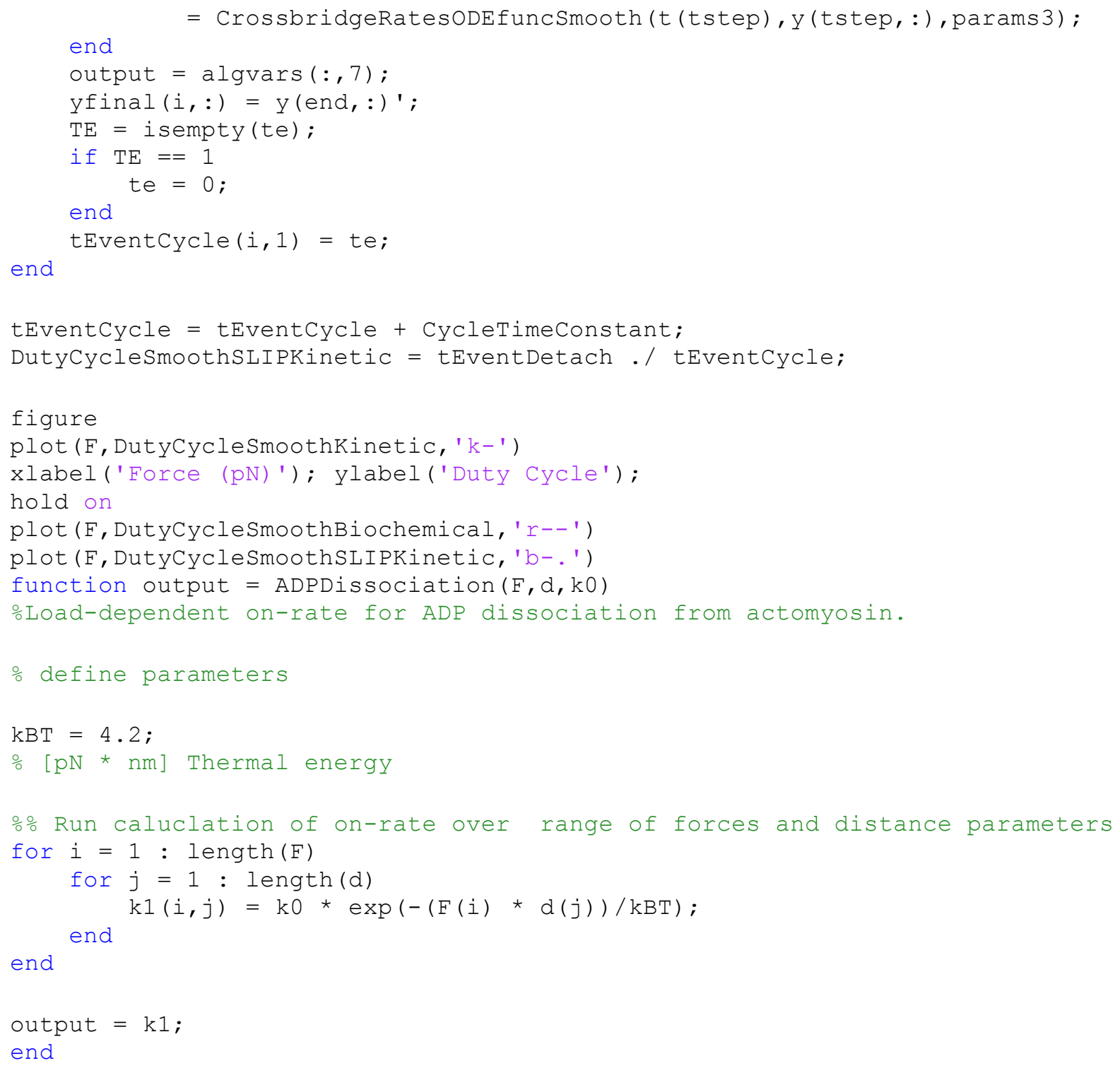




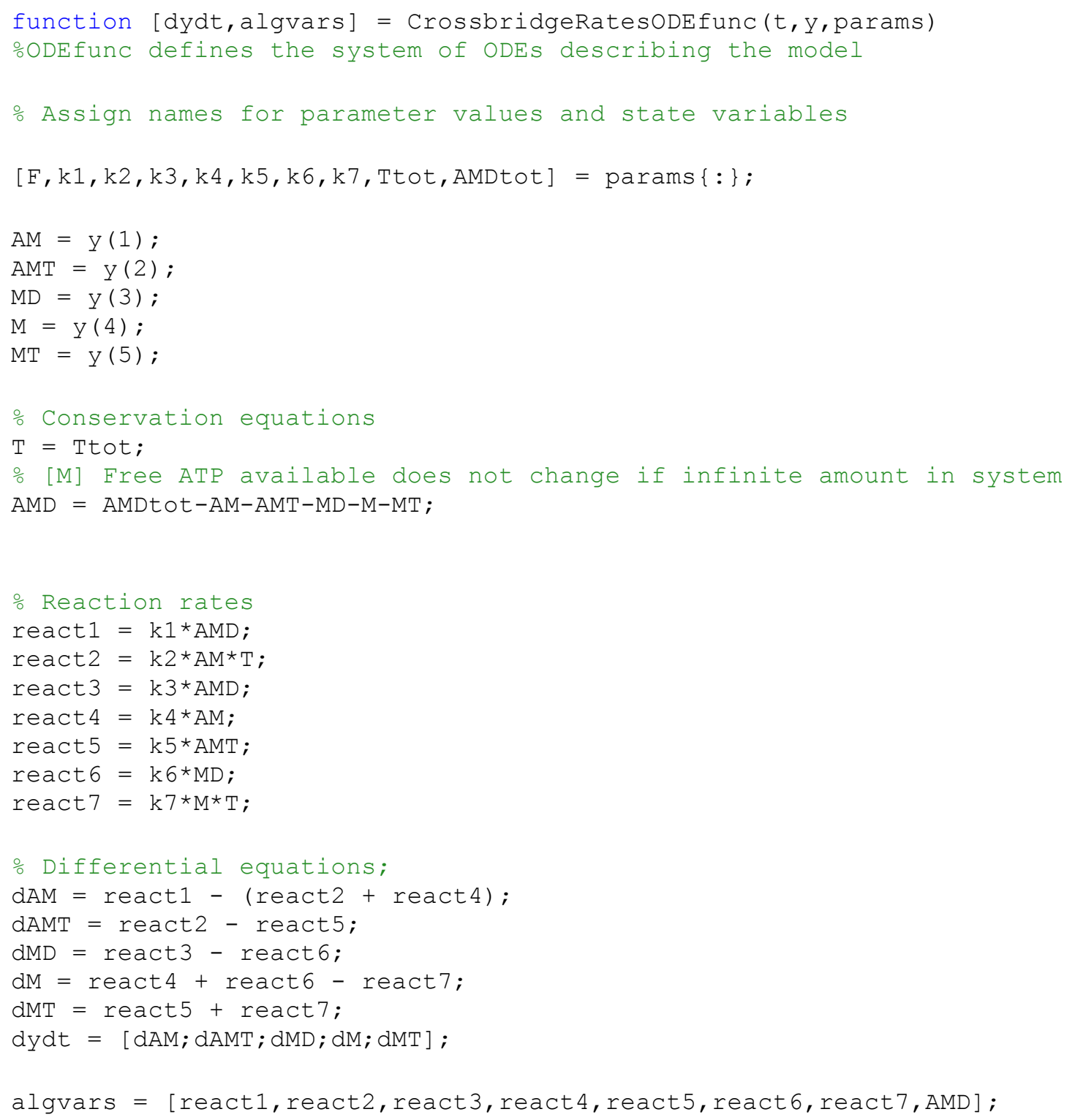




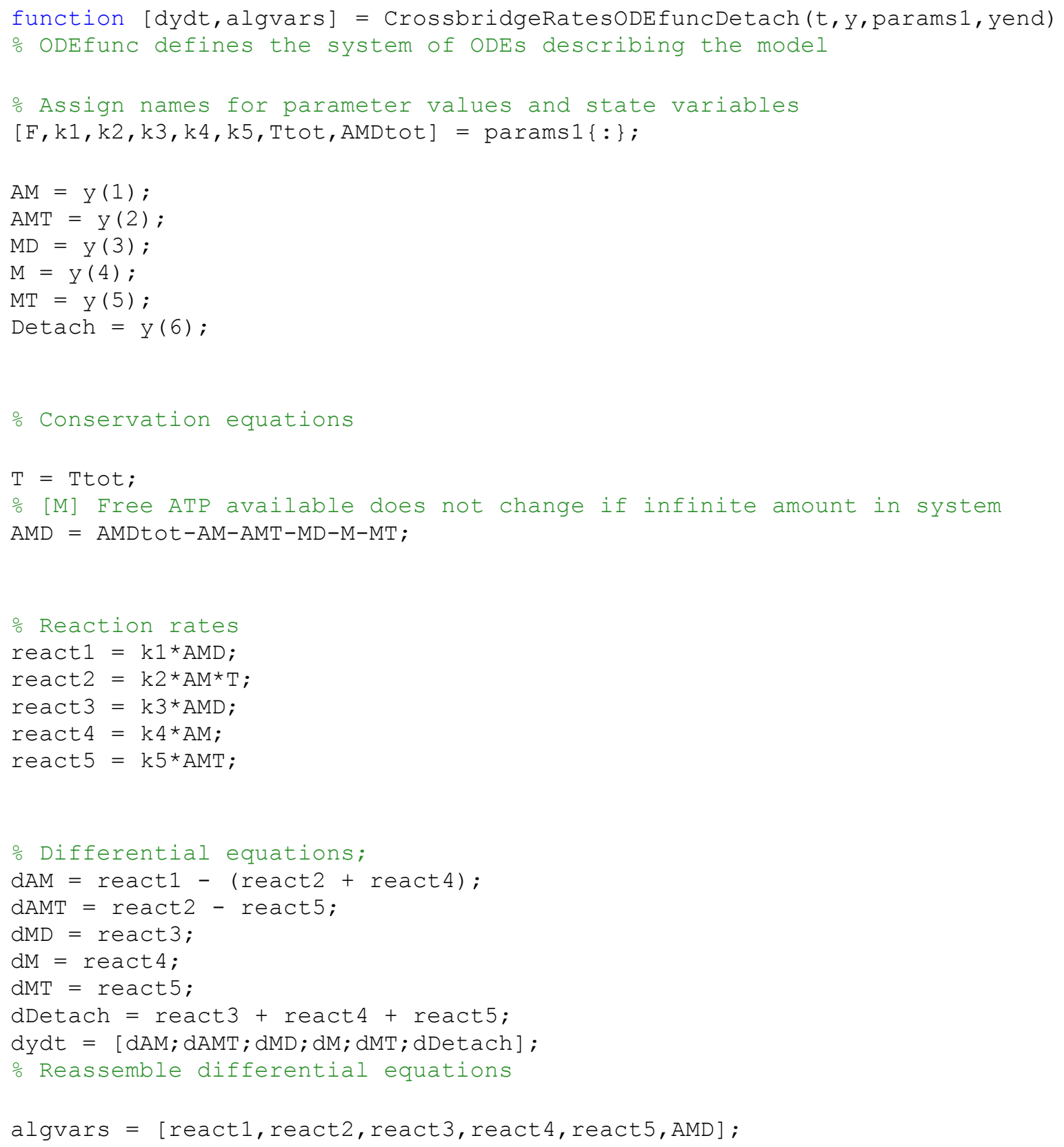




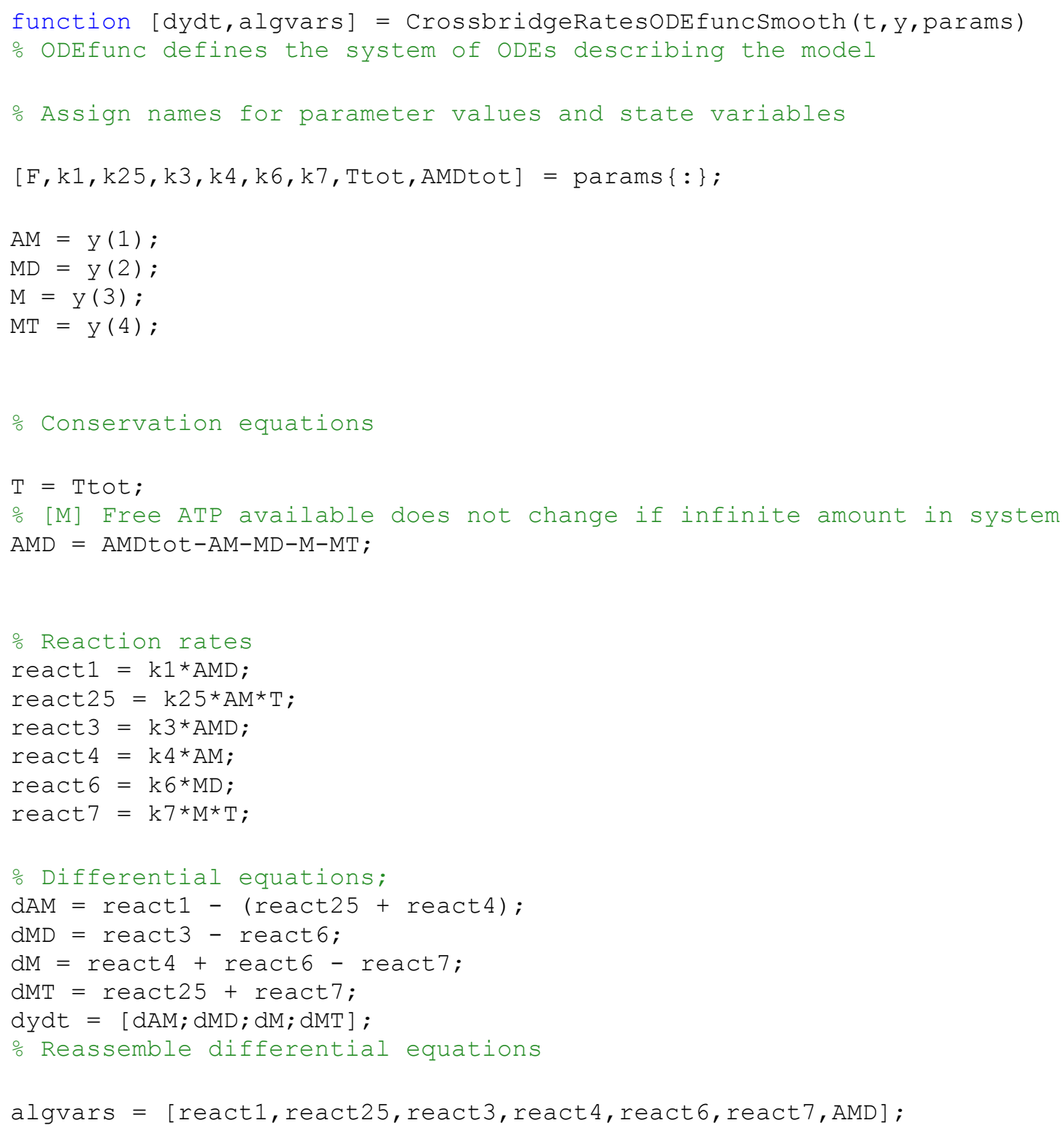




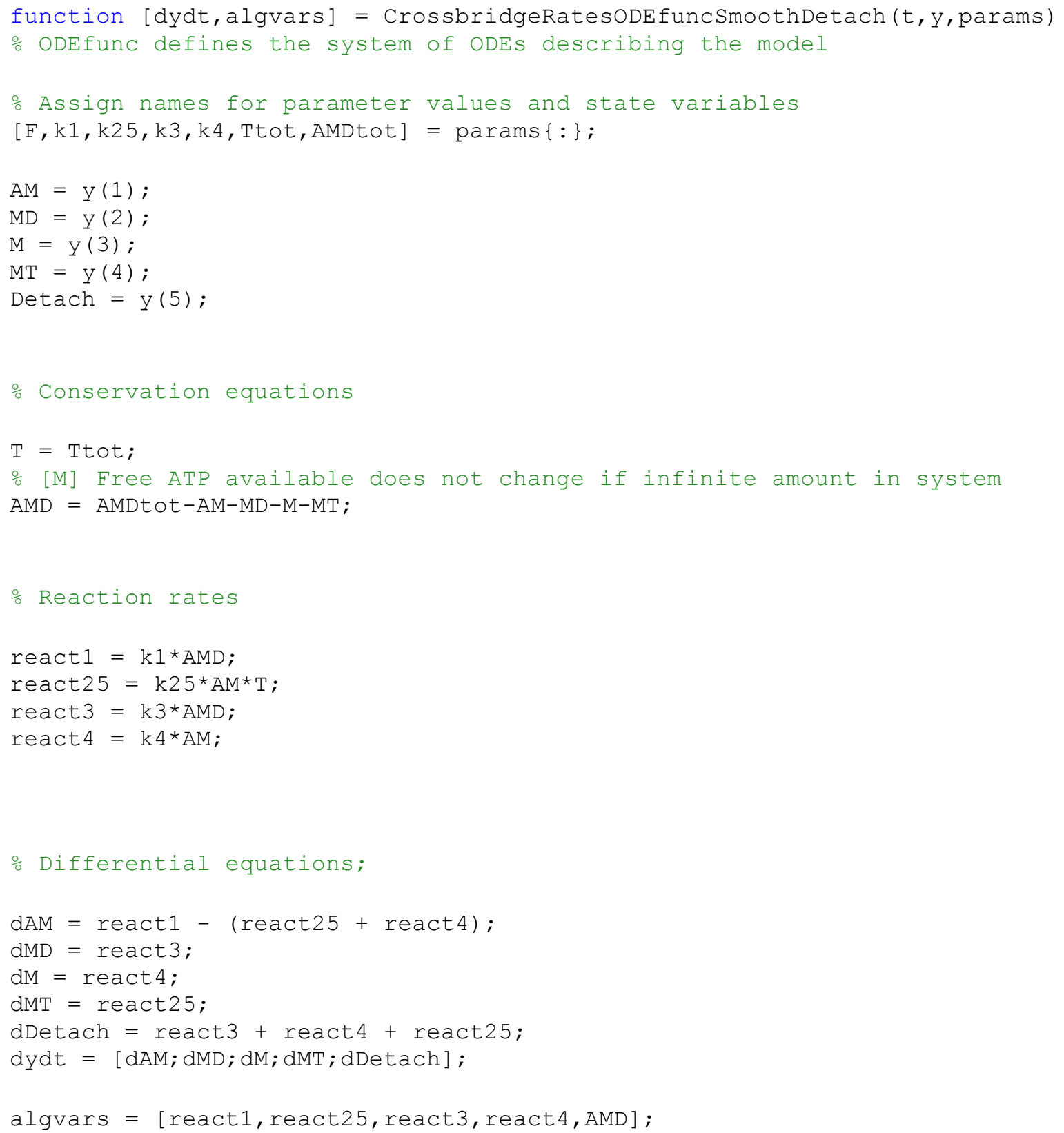


function [value, isterminal, direction] = events_AMC(t,y,params)

value $=y(5)-0.5 * 0.00418$;

isterminal $=0$;

direction $=0$; 
function [value, isterminal, direction] = eventsDetach_AMC(t,y,params)

value $=y(6)-(0.00418 * 0.5) ;$

isterminal $=0$;

direction $=0$; 
function [value, isterminal, direction] = eventsSmooth_AMC(t,y,params)

value $=y(4)-0.5 * 0.000352$;

isterminal $=0$;

direction $=0$; 
function [value, isterminal, direction] = eventsSmoothDetach_AMC(t,y,params)

value $=y(5)-(0.5 * 0.000352) ;$

isterminal $=0$;

direction $=0$; 


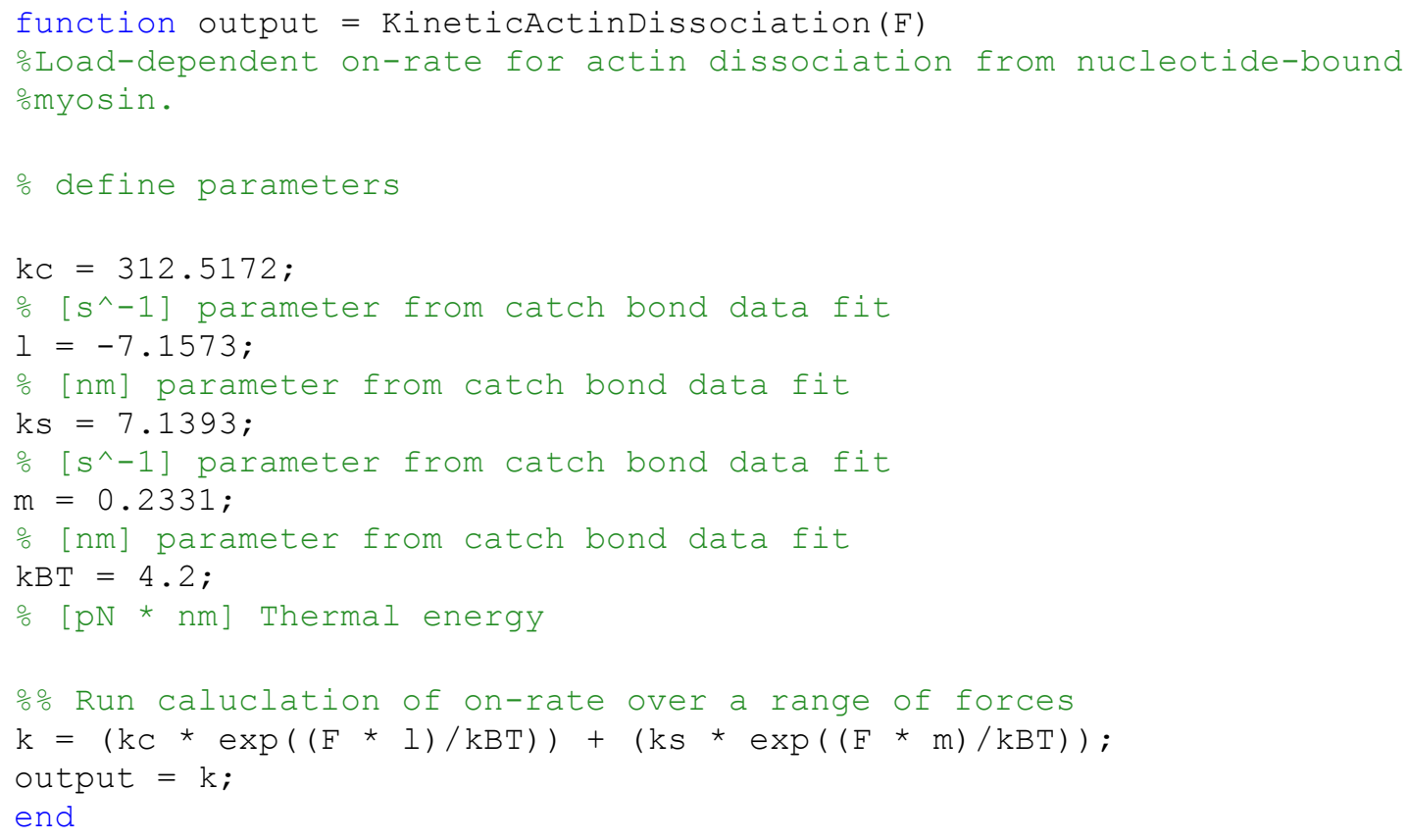




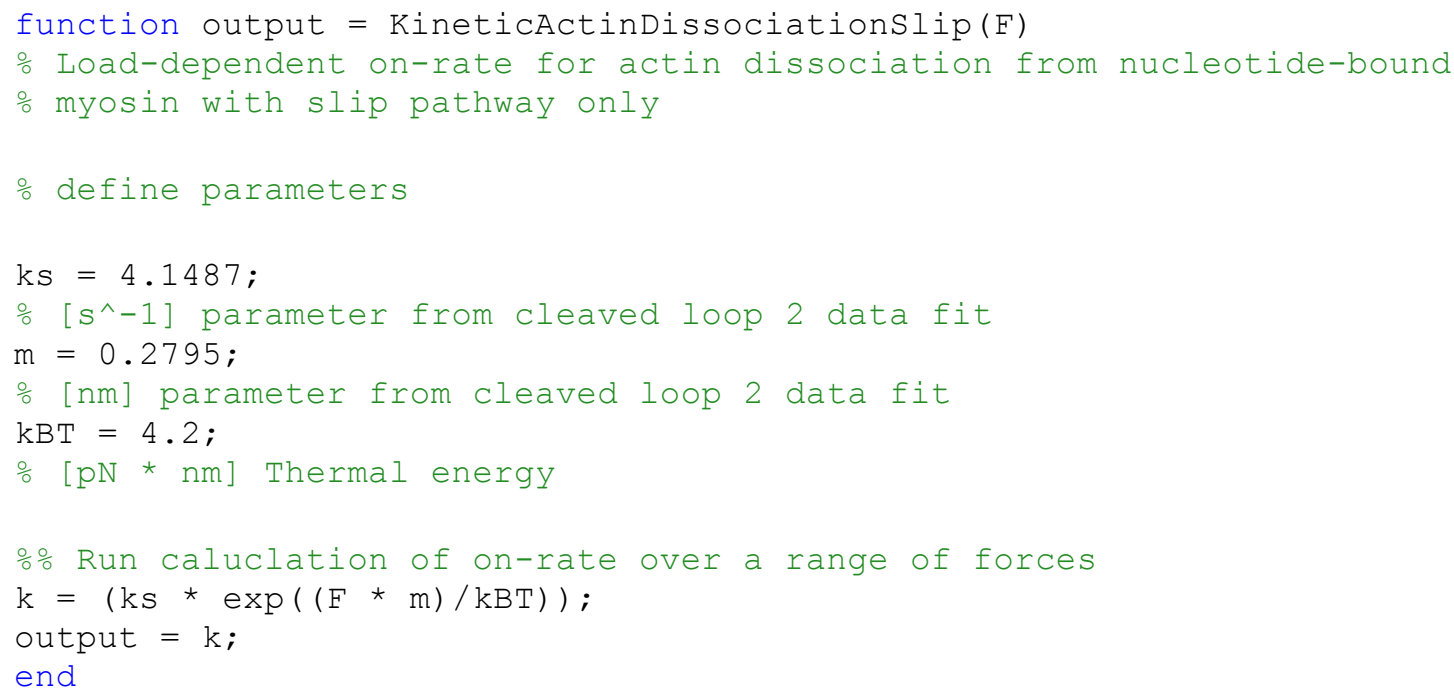

